by

\author{
MUSAMMAT MUTHMANNA ABDUL KADER \\ B.Sc. in Chemical Engineering \\ Ryerson University, Canada, 2016 \\ A thesis \\ presented to Ryerson University \\ in partial fulfilment of the requirements \\ for the degree of \\ Master of Applied Science \\ in the program of \\ Chemical Engineering
}

Toronto, Ontario, Canada, 2019

(c) Musammat Muthmanna Abdul Kader, 2019 


\section{AUTHOR'S DECLARATION}

I hereby declare that I am the sole author of this thesis. This is a true copy of the thesis, including any required final revisions, as accepted by my examiners.

I authorize Ryerson University to lend this thesis to other institutions or individuals for the purpose of scholarly research.

I further authorize Ryerson University to reproduce this thesis by photocopying or by other means, in total or in part, at the request of other institutions or individuals for the purpose of scholarly research.

I understand that my thesis may be made electronically available to the public. 


\title{
ABSTRACT \\ MANIPULATING THE MORPHOLOGY OF A METAL-ORGANIC FRAMEWORK: UiO-66
}

\author{
Musammat Muthmanna Abdul Kader \\ Master of Applied Science \\ Chemical Engineering
}

2019

Metal-Organic Frameworks (MOFs) are an emerging group of crystalline microporous materials that exhibit tunability towards various applications in gas storage, catalysts, and others. This project investigated the effects of reaction variables on UiO-66 via solvothermal synthesis. The results via the direct precipitation route at producing UiO-66 suggested that the crystallization rate was rather stagnant during the 30-day synthesis time, rendering the room temperature synthesis infeasible. Subsequently, the project investigated the effects of stirring time of the reactants for the first time in solvothermal synthesis, temperature, reaction time, modulator ratio, and reactant concentrations, on the morphology of UiO-66. Finally, the project studied the effects of adding glycols as co-solvents on UiO-66 crystal morphology. It was observed that only using ethylene glycol as a co-solvent successfully reformed the morphology of UiO-66 crystals from rounded octahedral crystals to anisotropic needle-like fibers. The results also indicated that diethylene glycol significantly improved the crystallinity of UiO-66 than triethylene glycol did. 


\section{ACKNOWLEDGEMENTS}

Words are powerless to thank God deeply for providing me strength, guiding me and encouraging me to face every situation that I have been through during the course of this project.

I would like to express heartfelt gratitude to my dear supervisors Dr. Chil-Hung Cheng and $\mathrm{Dr}$. Jianging $\mathrm{Wu}$ for helping me make this project possible. I would specially like to thank Dr. Chil-Hung Cheng for making me a part of his research project at Ryerson University. He has provided me with an invaluable optimistic learning experience where I have gained tremendous knowledge through his exercise of challenging details in the research field that helped me think critically and develop new ideas for my experiments. He has always supported me and believed in me despite the circumstances I was in. I am beyond grateful to have been doing my master's with a mentor like him.

I am thankful to the Department of Chemical Engineering at Ryerson University for continuous support for both academic and financial resources. I would also like to thank Dr. Amira AbdelRasoul for offering her selfless help in characterizing my samples. Special thanks to our MOF group who helped me in the laboratories at Ryerson for providing me with thought provoking suggestions and learning environment.

I would also like to thank the manager of the PXRD and SEM laboratory in the Earth Sciences department at University of Toronto, George Kretschmann, for his generosity in training me on these instruments. His valuable expertise, knowledge, and experience with characterization of microporous materials has helped my project.

Thanks to the technical assistant at the Ryerson University Analytical Centre, Shawn McFadden, for helping me with FTIR analysis of my samples.

I sincerely appreciate everyone who offered me their support in completing my thesis and went unmentioned. 


\section{TABLE OF CONTENTS}

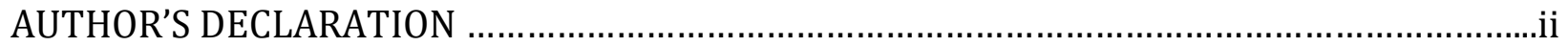

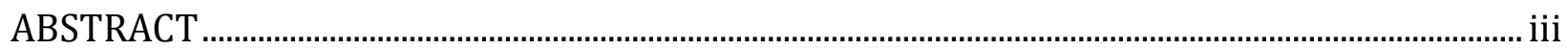

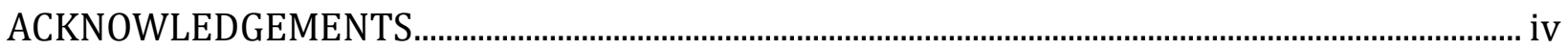

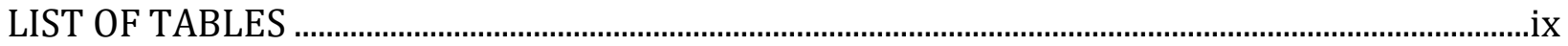

LIST OF FIGURES

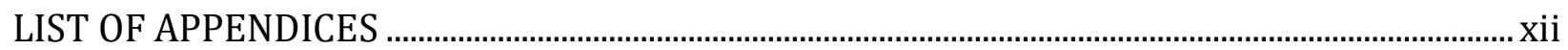

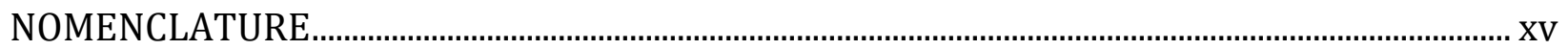

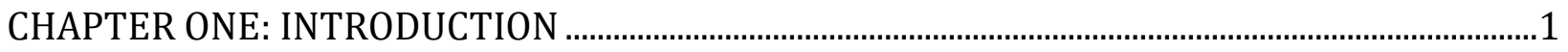

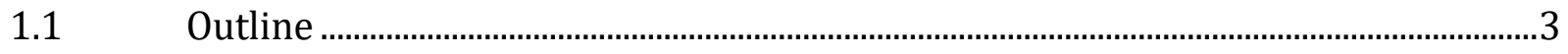

CHAPTER TWO: LITERATURE REVIEW ……...................................................................................

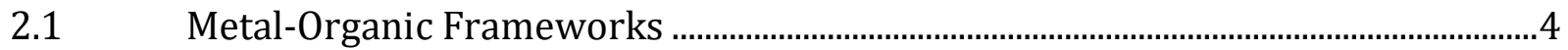

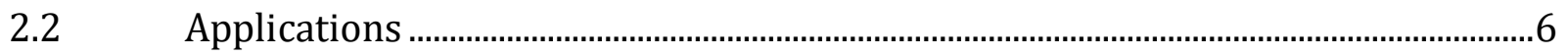

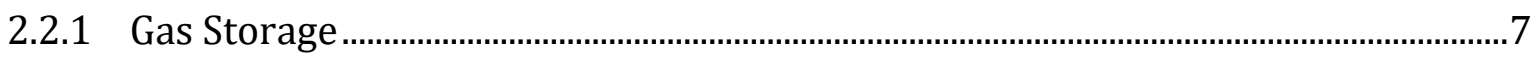

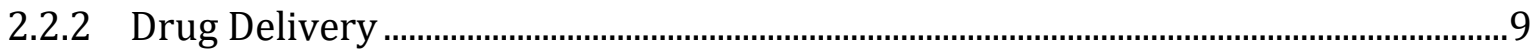

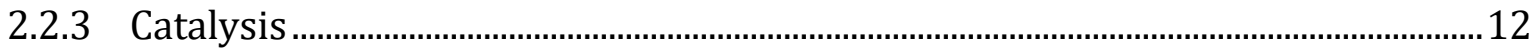

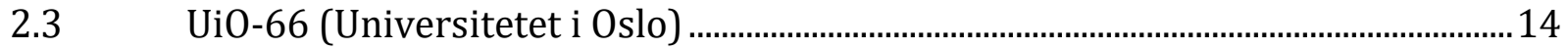

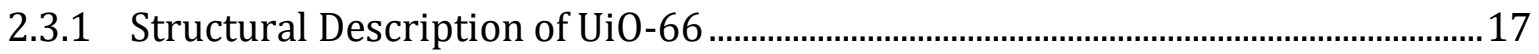

2.3.2 Mechanical Strength and Chemical Stability of UiO-66 ..........................................18

2.4 Synthesis Methods of Metal Organic Frameworks...................................................23

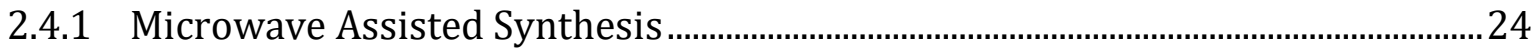

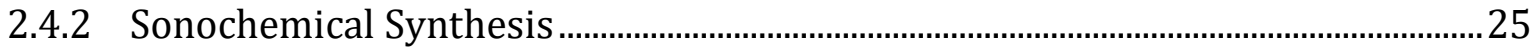

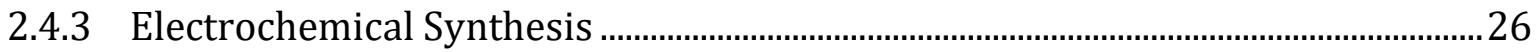

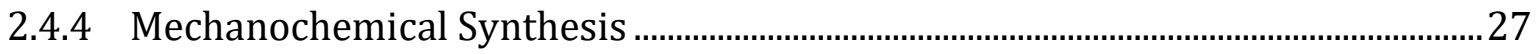

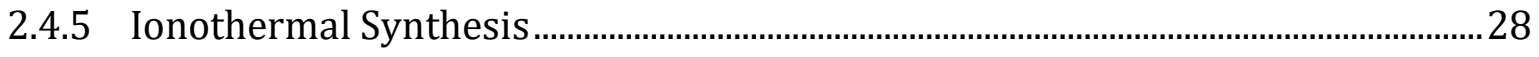


2.4.6 Conventional Solvothermal Synthesis ………………...............................................29

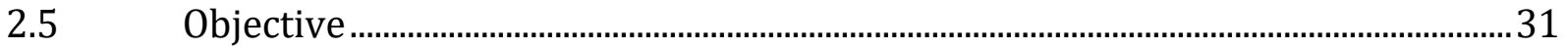

CHAPTER THREE: MATERIALS AND METHODS ………....................................................................

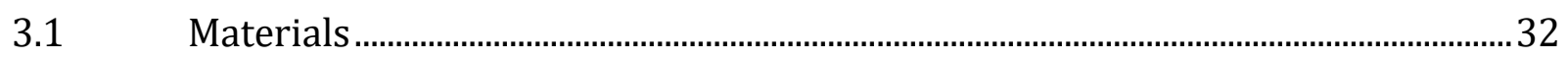

3.2 Synthesis Routes ......................................................................................................

3.2.1 Synthesis of UiO-66 without co-solvent .........................................................................32

3.2.2 Synthesis of UiO-66 with co-solvent................................................................................

3.3 Experimental Methods .....................................................................................................36

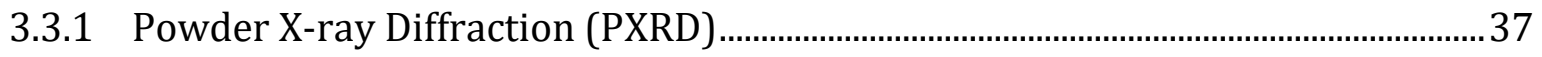

3.3.2 Scanning Electron Microscope (SEM) ……………………………………………......

3.3.3 Attenuated Total Reflection Infrared (ATR-IR) ............................................................40

3.3.4 Thermogravimetric Analysis (TGA) and Differential Scanning Calorimetry (DSC) $\ldots \cdots \cdots \cdots \cdots \cdots \cdots \cdots \cdots \cdots \cdots \cdots \cdots \cdots \cdots \cdots \cdots \cdots \cdots \cdots \cdots \cdots \cdots \cdots \cdots \cdots \cdots \cdots \cdots \cdots \cdots \cdots \cdots \cdots \cdots \cdots \cdots \cdots \cdots \cdots \cdots \cdots \cdots \cdots \cdots \cdots \cdots \cdots \cdots \cdots \cdots \cdots \cdots \cdots \cdots \cdots \cdots \cdots \cdots \cdots \cdots$

3.3.5 Brunauer Emmett and Teller (BET) Surface Area Measurements..........................42

CHAPTER FOUR: FACTORS INFLUENCING THE MORPHOLOGY OF MOFs...................................44

4.1 DIRECT PRECIPITATION SYNTHESIS ……………………………………………....... 44

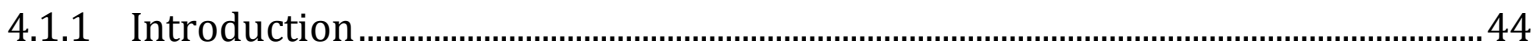

4.1.2 Results and Discussion .......................................................................................................4

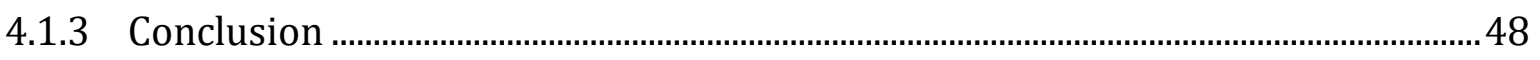

4.2 EFFECT OF STIRRING TIME ......................................................................................

4.2.1 Introduction ....................................................................................................................

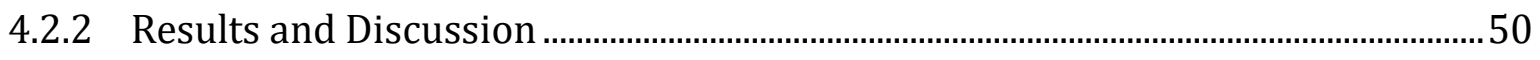

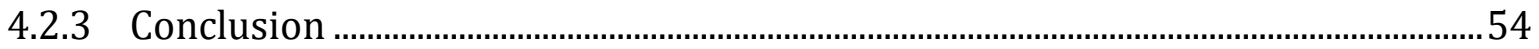

4.3 EFFECT OF REACTION TIME …………………......................................................5

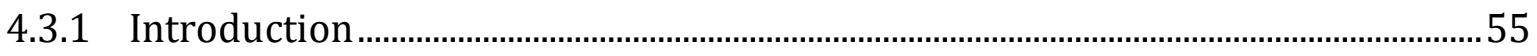


4.3.2 Results and Discussion ................................................................................................57

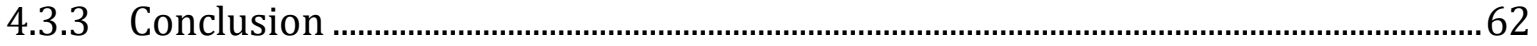

4.4 EFFECT OF MOLAR RATIO OF Zr:BDC ………..........................................................63

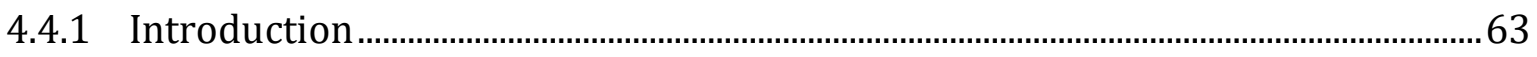

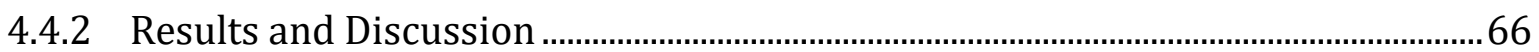

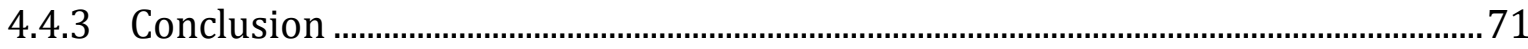

4.5 EFFECT OF REACTION TEMPERATURE..........................................................................71

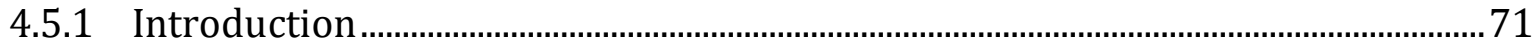

4.5.2 Results and Discussion .......................................................................................................74

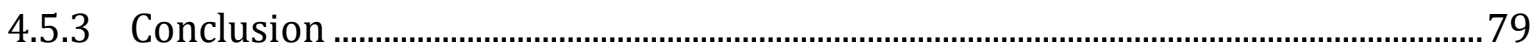

4.6 EFFECT OF MODULATOR CONCENTRATION ...............................................................79

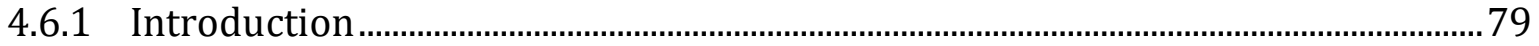

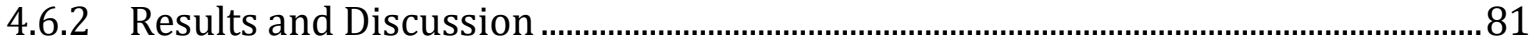

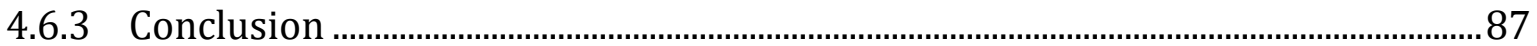

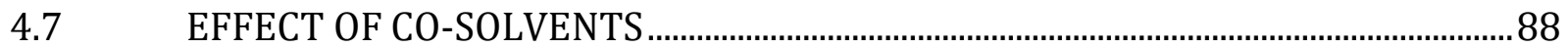

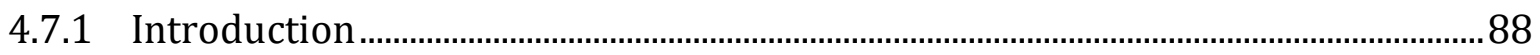

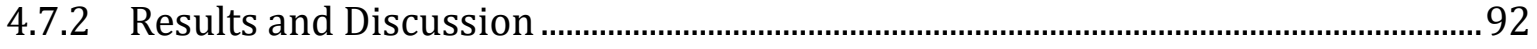

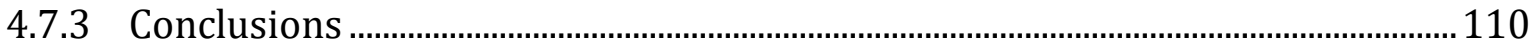

4.8 EFFECT OF THE CONCENTRATION OF GLYCOLS....................................................111

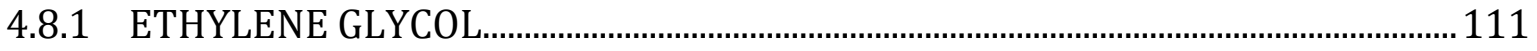

4.8.2 DIETHYLENE GLYCOL................................................................................................119

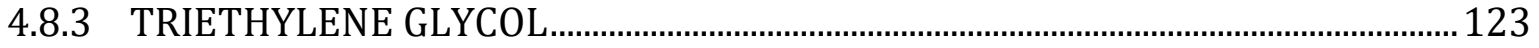

4.9 EFFECT OF MODULATOR ON EG AIDED UiO-66......................................................128

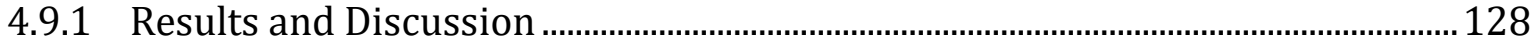

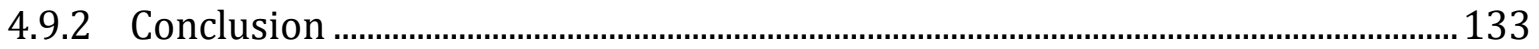


CHAPTER FIVE: SUMMARY.

CHAPTER SIX: RECOMMENDATIONS

APPENDICES .

REFERENCES

145 


\section{LIST OF TABLES}

Table 2.2.1-1: Summary of hydrogen gas uptake capacities of Zr-based MOFs.........................8

Table 2.3-1: Remarkable Zr-based MOFs with exceptional surface areas ...................................15

Table 3.1-1: Materials used for the synthesis of UiO-66.................................................................32

Table 4.2.2-1: Reactant composition and conditions to investigate the effect of stirring time

Table 4.3.2-1: Reactant composition and conditions to investigate the effect of reaction time 57

Table 4.4.2-1: Reactant composition and conditions to investigate the effect of metal:linker ratio. .65

Table 4.5.2-1: Reactant composition and conditions to investigate the effect of reaction temperature .74

Table 4.6.2-1: Reactant composition and reaction conditions to investigate the effect of modulator concentration. 81

Table 4.7.2-1: Reactant composition and reaction conditions to investigate the effect of co-

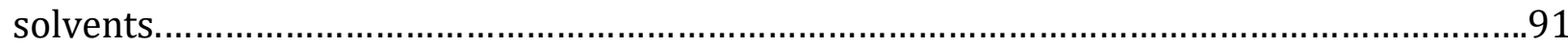

Table 4.7.2-2: Calculated CPO values based on reflections $\mathrm{X}$ and $\mathrm{Y}$...............................................95

Table 4.7.2-3: Vibrational modes of the IR Spectrum of UiO-66 reported in literature..........97

Table 4.7.2-4: A comparison of the vibrational modes $\left(\mathrm{cm}^{-1}\right)$ for the UiO-66 samples synthesized to examine the effect of glycols ${ }^{\mathrm{a}}$.....................................................................................98

Table 4.8.1.1-1: Reactant composition and reaction conditions to investigate the effect of EG concentration 110

Table 4.8.1.1-2: Vibrational frequencies for Ui0-66 samples synthesized with 10 eq., 20eq., and 30 eq. EG.

Table 4.8.2.1-1: Reactant composition and reaction conditions to investigate the effect of DEG concentration.

Table 4.8.3.1-1: Reactant composition and reaction conditions to investigate the effect of TEG concentration.

Table 4.9.1-1: Reactant composition and reaction conditions to investigate the role of acetic acid in EG synthesized UiO-66 crystals. 128 


\section{LIST OF FIGURES}

Figure 1-1: Performance of mixed matrix membranes in selective permeation of $\mathrm{CO}_{2}$ in a

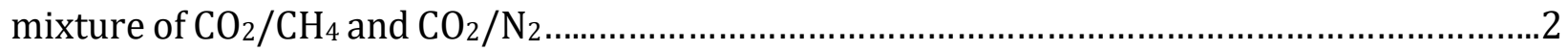

Figure 2.1-1: Molecular structure of various MOFs ……..................................................

Figure 2.3.1-1: Structure of UiO-66 MOF. Colors red, grey, blue and white represent zirconium, carbon, oxygen, and hydrogen atoms, respectively. The grey, blue and white structures are the BDC linkers connecting the SBUs (red, grey, blue). Illustrated are (a) Tetrahedral cage. (b) Octahedral cage. (c) $8 \mathrm{Zr}_{6} \mathrm{O}_{4}(\mathrm{OH})_{4}$ bricks formed by combination of one octahedral cage and two adjacent tetrahedral cages forming a cubic unit with yellow arrows representing the squeezing direction that the $\mathrm{Zr}_{6} \mathrm{O}_{6}$ octahedron undergoes after the dehydroxylation process. Yellow circles point perpendicular to the page ............................ 17 Figure 2.3.2-1: Thermal stability of UiO-66: a) PXRD profiles of UiO-66 with increasing activation temperature in air b) TGA curve of UiO-66 in air c), d), and e) MS results of water, DMF, benzene, respectively..........................................................................................................20 Figure 2.3.2-2: Structural stability of UiO-66: a) PXRD profiles of desolvated UiO-66 in the indicated solvents at room temperature in various stirred suspension solutions for $24 \mathrm{~h}, \mathrm{~b}$ ) PXRD profiles of desolvated UiO-66 in the indicated solvents at room temperature in various stirred suspension solution for 2 hours. The arrow in part (a) is the $\mathrm{Si}(111)$ reflection at $2 \theta$

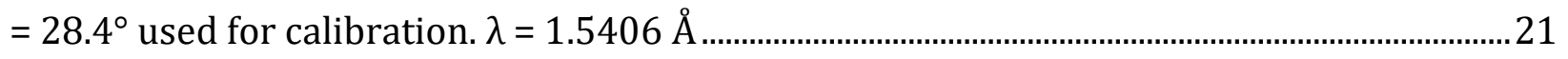
Figure 2.4-1: Yearly increase in reported Zr-MOFs ........................................................................23

Figure 2.4.5-1: Structural representation of 1-alkyl-3-methylimidazolium...............................28 Figure 3.2.2-1: Schematic diagram of the experimental set up for solvothermal synthesis of

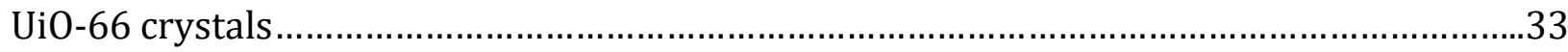

Figure 3.2.1-2: Formulated UiO-66 crystals before drying (left) and after drying(right)......34

Figure 3.2.2-3: Schematic diagram of typical solvothermal synthesis of UiO-66 crystals......36

Figure 3.3.1-1: Schematic representation of Bragg's law of diffraction........................................38

Figure 3.3.1-2: PXRD pattern of UiO-66

Figure 3.3.2-1: SEM image of as-synthesized UiO-66 sample. …………………………………......39

Figure 3.3.3-1: FTIR Spectrum of as-synthesized UiO-66. .............................................................. 41

Figure 3.3.4-1: TGA(green) and DSC(blue) profiles of as-synthesized UiO-66........................... 42 
Figure 4.1.2-1: SEM images of UiO-66: a) synthesized using the direct precipitation method,

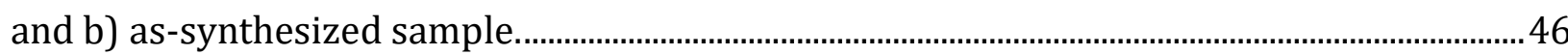

Figure 4.1.2-2: PXRD pattern of UiO-66 (black) and desolvated (grey) UiO-66....................47 Figure 4.1.2-3: Comparison of XRD patterns of UiO-66 synthesized at room temperature and at $120^{\circ} \mathrm{C}$. 47

Figure 4.2.2-1: SEM images of UiO-66 with stirring times at a) $6 \mathrm{~h}, \mathrm{~b}$ ) $12 \mathrm{~h}, \mathrm{c}) 24 \mathrm{~h}$, and d) $48 \mathrm{~h}$.

Figure 4.2.2-2: XRD patterns on varying stirring times for $6 \mathrm{~h}, 12 \mathrm{~h}, 24 \mathrm{~h}$, and $48 \mathrm{~h} . . . \ldots \ldots \ldots \ldots . . . . . . .52$

Figure 4.2.2-3: Effect of stirring time on the crystallinity of UiO-66 crystals.......................53

Figure 4.3.2-1: PXRD patterns on varying reaction times after 48 hours of stirring................58

Figure 4.3.2-2: SEM images of UiO-66 with reaction times after $48 \mathrm{~h}$ of stirring at a) $12 \mathrm{~h}, \mathrm{~b}$ ) $24 \mathrm{~h}, \mathrm{c}) 48 \mathrm{~h}, \mathrm{~d}) 72 \mathrm{~h}$, and e) as-synthesized. 59

Figure 4.3.2-3: Effect of reaction time and extended stirring time on the crystallinity of UiO66. 60

Figure 4.4.1-1: Schematic representation of effect of change in metal:ligand concentration..

Figure 4.4.1-2: Distinct frameworks synthesized from Zn-based MOF with varying co-ligand concentration as a) $0.1 \mathrm{mmol}$, b) $0.2 \mathrm{mmol}$, and c) $0.4 \mathrm{mmol}$. 65

Figure 4.4.2-1: PXRD pattern of UiO-66 with $\operatorname{Zr}$ (metal) as a limiting reactant. .67

Figure 4.4.2-2: SEM images of UiO-66 with $\mathrm{Zr}$ (metal) as a limiting reactant: a) as-synthesized

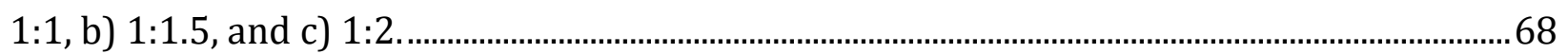

Figure 4.4.2-3: PXRD pattern of UiO-66 with BDC(ligand) as a limiting reactant. .69

Figure 4.4.2-4: SEM images of UiO-66 with BDC(ligand) as a limiting reactant: a) as-

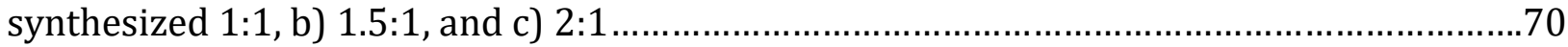

Figure 4.5.1-1: Coordination modes of $\mathrm{cpdba}^{2-}$ ligand with atoms indicated as pink $=\mathrm{Mn}$, red $=\mathrm{O}$, grey $=\mathrm{C}, \mathrm{H}$, and blue $=\mathrm{N}$ atoms where a) $140^{\circ} \mathrm{C}$ (bridging bidentate coordination), b) $150^{\circ} \mathrm{C}$ (no dominant bridging bidentate) , and c) $170^{\circ} \mathrm{C}$ (monodentate)...................................71

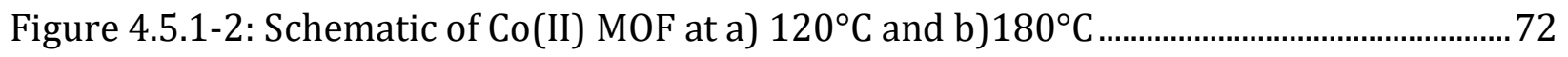
Figure 4.5.1-3: Conformation of titmb ligand and coordination geometry of Co(II) indicating $\mathrm{N}, \mathrm{C}, \mathrm{O}$ atoms in blue, grey, and red, respectively .72 
Figure 4.5.2-1: XRD patterns of UiO-66 for with varying temperatures.

Figure 4.5.2-2: SEM images of UiO-66 produced at a) $100^{\circ} \mathrm{C}$ for $\left.48 \mathrm{~h} \mathrm{~b}\right) 120^{\circ} \mathrm{C}$ for $24 \mathrm{~h}, \mathrm{c}$ ) $140^{\circ} \mathrm{C}$

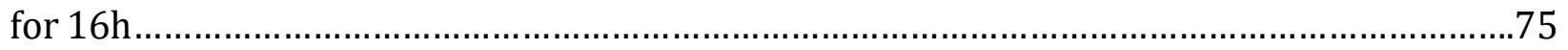

Figure 4.6.2-1: PXRD pattern of UiO-66 for different acetic acid concentrations. ...................81

Figure 4.6.2-2: SEM images of UiO-66 at acetic acid concentrations at a) 0.0103mol, b)

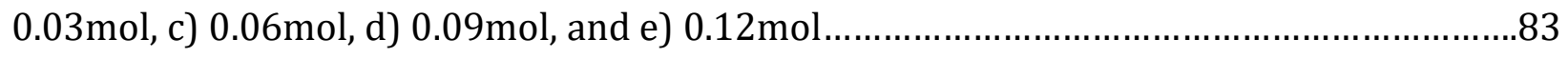

Figure 4.6.2-3: Effect of modulator concentration on the crystallinity of UiO-66................84

Figure 4.7.1-1:Different morphologies of MIL-96(Al) with various cosolvents .........................88

Figure 4.7.1-2: The self-assembly of $\mathrm{Mg}\left(\mathrm{NO}_{3}\right)_{2} \cdot 6 \mathrm{H}_{2} \mathrm{O}$ and 3,5-PDC under different solvents [108] 89

Figure 4.7.1-3: Coordination of $\mathrm{Cd}\left(\mathrm{NO}_{3}\right)_{2} \cdot 4 \mathrm{H}_{2} \mathrm{O}$ and $\mathrm{H}_{3}$ bpt by using different solvents. ........90

Figure 4.7.2-1: PXRD Pattern of samples produced with 30 eq. glycols. 93

Figure 4.7.2-2: Miller indices of as-synthesized UiO-66 versus UiO-66 synthesized with EG as co-solvent. .94

Figure 4.7.2-3: SEM images of samples produced with 30 eq. a)EG, b)DEG, c)TEG d)assynthesized (no co-solvent)... .96

Figure 4.7.2-4: FTIR spectrum reported on the same wavenumber range for UiO-66 samples synthesized using co-solvent EG, DEG, and TEG. Scaling of the spectra has been omitted for clarity. .98

Figure 4.7.2-5: Schematic representation of a) thermodynamically labile regions of UiO-66 b) configuration of UiO-66 in (111) plane c) configuration of UiO-66 in (200) plane..........101 Figure 4.7.2-6: Illustration of the production of in-situ ligand BHET and subsequent oriented growth of UiO-66 crystals in the (200) with 30-eq. EG co-solvent............................................104 Figure 4.7.2-7: Adsorption isotherm log plot of 30-EG-UiO-66..................................................... 105 Figure 4.7.2-8: TGA(green) and DSC(blue) profiles of 30-EG-UiO-66...................................... 107 Figure 4.8.1.1 -1: XRD Pattern of samples produced varying EG concentrations. ..................112 Figure 4.8.1.1-2: SEM images of samples produced with EG of a) 5eq. b) 10eq., c) 20eq., d) 30 eq. e) 40 eq., and e) 50 eq.

Figure 4.8.1.1-3: FTIR spectra of UiO-66 synthesized with different concentrations of EG as co-solvent. 114

Figure 4.8.2.1-1: XRD patterns for UiO-66 samples with varying DEG concentrations.......119 
Figure 4.8.2.1-2: SEM images of samples produced with DEG of a) 10eq., b) 20eq., and c) 30 eq.

Figure 4.8.2.1-3: Effect of DEG concentration on the crystallinity of UiO-66 particles........121 Figure 4.8.3.1-1: XRD patterns for UiO-66 samples with varying TEG concentrations........124 Figure 4.8.3.1-2: SEM images of samples produced with TEG of a) 5eq. b) 10eq., c) 20eq., d) 30 eq. 125

Figure 4.8.3.1-3: Effect of TEG concentration on the crystallinity of UiO-66 particles.........126 Figure 4.9.1-1: XRD patterns illustrating the effect of modulator on the morphology of assynthesized and EG aided UiO-66 crystals. 129

Figure 4.9.1-2: SEM images of UiO-66 samples: a) without EG and acetic acid, b) without EG with acetic acid, c) with EG without acetic acid, and d) with EG and with acetic acid.........130

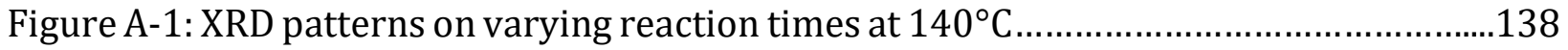
Figure A-2: SEM images of UiO- 66 at $140^{\circ} \mathrm{C}$ with reaction times of a) $12 \mathrm{~h}$, b) $24 \mathrm{~h}$ c) $16 \mathrm{~h}$, and d) as-synthesized. 139

Figure B-1: XRD patterns of UiO- 66 at $120^{\circ} \mathrm{C}$ for $96 \mathrm{~h}$ with 30 eq. of various glycols...........140 Figure B-2: SEM images of UiO- 66 at $120^{\circ} \mathrm{C}$ for $96 \mathrm{~h}$ with 30 eq. of a) EG, b) DEG, and c) TEG 141

Figure C-1: SEM image of UiO-66 at $120^{\circ} \mathrm{C}$ for $96 \mathrm{~h}$ using 30 eq. EG co-solvent......................142

Figure C-2: XRD image of UiO- 66 at $120^{\circ} \mathrm{C}$ for $96 \mathrm{~h}$ using 30 eq. EG co-solvent.....................142

Figure C-3: XRD image of UiO-66 at $120^{\circ} \mathrm{C}$ for $96 \mathrm{~h}$ using 30 eq. EG co-solvent. 143 


\section{LIST OF APPENDICES}

APPENDIX A: Effect of reaction time at $140^{\circ} \mathrm{C}$ with 24 hours of stirring via Scheme 1 ......138 APPENDIX B: Effect of 30 eq. co-solvents with $24 \mathrm{~h}$ stirring at $120^{\circ} \mathrm{C}$ for $96 \mathrm{~h}$ via Scheme

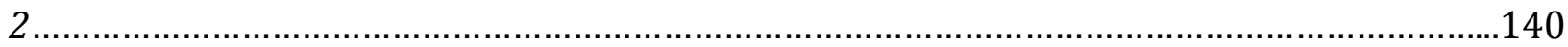

APPENDIX C: Reproducibility of needle-shaped fibers using EG as a co-solvent with 24h

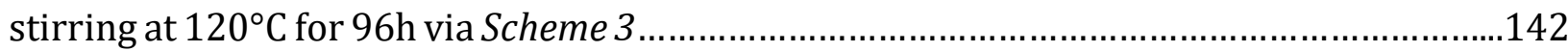




\section{NOMENCLATURE}

\section{Compound Name Acronyms}

[Omim $] \mathrm{Cl}$

[Hmim]Cl

$[\mathrm{Dmim}] \mathrm{Cl}$

1,2,3- $\mathrm{H}_{3} \mathrm{btc}$

1,3,5-H3btc

2,2'-bpy

3,5-pdc

BHET

bpdc $^{2-}$

btc

$\mathrm{H}_{2} \mathrm{bdc}$

$\mathrm{H}_{2}$ bdc- $(\mathrm{OH})_{2}$

$\mathrm{H}_{2}$ cpdba

$\mathrm{H}_{3}$ bpt

py- $\mathrm{xp}^{4-}$

ptba $^{4-}$

py-ptp ${ }^{4-}$

por-ptp ${ }^{4-}$

tbapy ${ }^{4-}$

titmb

tpdc ${ }^{2-}$ 1-octyl-3-methylimidazolium chloride

1-hexyl-3-methylimidazolium chloride

1-decyl-3-methylimidazolium chloride

1,2,3-benzenetricarboxylic acid

1,3,5-benzenetricarboxylic acid

2,2'-bipyridine

3,5-pyrdinedicarbox

bis(2-hydroxyethyl) terephthalate

biphenyl-4,40-dicarboxylate

benzene-1,3,5-tricarboxylic acid

terephthalic acid

2,5-dihydroxyterephthalic acid

4-(4-carboxyphenylamino)-3,5-dinitrobenzoic acid

biphenyl-3,4,5-tricarboxylic acid

4',4'”,4'”'”,4"','”'-(pyrene-1,3,6,8-tetrayl)tetrakis(2',5'-dimethyl-[1,1'biphenyl]-4-carboxylate)

4-[2-[3,6,8-tris[2-(4-carboxylatephenyl)-ethynyl]-pyren-1yl]ethynyl]-benzoate

4,4',4",4"')-((pyrene-1,3,6,8-tetrayltetrakis(benzene4,1diyl))tetrakis(ethyne-2,1-diyl))tetrabenzoate

meso-tetrakis-(4-((phenyl)ethynyl)benzoate)porphyrin

1,3,6,8-tetrakis(p-benzoate)pyrene

1,3,5-tri(imidazol-1-ylmethyl)-2,4,6-trimethylbenzene

$\left[1,1^{\prime}: 4^{\prime}, 1^{\prime \prime}\right.$ - terphenyl]-4,4"'-dicarboxylate 


\section{General Acronyms}

${ }^{1}$ HNMR

a.u.

BET

$\csc$

DEF

DEG

DMA

DMF

EG

EXAFS

fcu

FTIR

$\mathrm{ftw}$

MA

MOF

PXRD

SAXS

SBU

SEM

TBU

$\mathrm{TEABr}$

TEG

TGA

UiO-66

XRD

$\mathrm{Zr}$ proton nuclear magnetic resonance

arbitrary unit

Brunauer-Emmett-Teller

combination of square and cube geometry

diethylformamide

diethyleneglycol

dimethylaluminum

dimethylformamide

ethylene glycol

extended X-ray absorption fine structure

face-centered cubic unit

Fourier transform infrared spectroscopy

combination of cuboctahedral and square geometry

mass spectroscopy

metal-organic framework

powder X-ray diffraction

small-angle X-ray scattering

secondary building unit

scanning electron microscope

tertiary building unit

tetraethylammonium bromide

triethyleneglycol

thermogravimetric analysis

Universitetet i Oslo (University of Oslo)

X-ray diffraction

zirconium 


\section{Symbols}

\begin{tabular}{ll}
$v_{m}$ & adsorbed monolayer volume \\
$v$ & adsorbed volume of gas \\
$\theta$ & angle of the incident X-rays \\
$\mathrm{A}$ & area of cross-sectional of a single adsorbed gas molecule \\
$\mathrm{N}$ & Avogadro's number \\
$\mathrm{C}$ & BET constant \\
$a$ & edge length of a unit cell \\
$P$ & equilibrium gas pressure \\
$\mu_{3}-\mathrm{OH}$ & hydroxo ligand connecting 3 metal ions \\
$d_{h k l}$ & interplanar distance between planes \\
$\mathrm{M}$ & mass of materials \\
$h k l$ & Miller indices \\
$\rho$ & overall electron density function \\
$\mu_{3}-\mathrm{O}$ & oxo ligand connecting 3 metal ions \\
$\rho$ & rocking vibration \\
$p_{0}$ & saturation pressure \\
$\nu$ & stretching vibration \\
$\Psi$ & wave function of an electron \\
$\lambda$ & wavelength of the X-rays \\
\hline &
\end{tabular}




\section{CHAPTER ONE}

\section{INTRODUCTION}

MOFs are an emerging group of crystalline porous materials that have gained popularity in the last decade because of their ability to be fashioned or tailored depending on the need of the application. These compounds differ in various properties such as structure and stability depending on the synthesis method employed, resulting in virtually innumerable MOFs. The dynamic field is theoretically reported to have simulated more than 100,000 MOFs, out of which 20,000 successfully synthesized MOFs are primarily differentiated by the type of metal centers and organic linkers, which in turn causes variation in their pore dimension, pore size, crystal shape and size, chemical stability, and framework functionality [1], [2]. With permanent porosities, large surface areas, and potential tunability, MOFs have demonstrated potential implementation in post-combustion $\mathrm{CO}_{2}$ capture [3], catalysis [4], molecule adsorption and separation [4], [5], drug delivery [4], and hydrogen storage [1], [3], [5], [6].

Recent articles have reported the production of a new composite material known as MMM or mixed-matrix membrane, which comprises of a MOF material incorporated into a polymer matrix. These materials offer high separation efficiency, and better permeability and selectivity trade off than pure matrix membrane [7]. An MMM is a composite material that combines the constructive features of MOFs, i.e., their large surface areas, high thermal and chemical stability, and permanent porosities, with the constructive features of polymers such as easy processability, robust polymer matrices and good separation efficiencies, by embedment of MOFs into the polymer matrix via chemical interaction of active sites of the polymer with the free carboxylate groups of UiO-66 [1], [7].The first MMM was produced by Kaliaguine and co-workers with UiO-66(Zr) and UiO-66(Zr)- $\mathrm{NH}_{2}$ individually embedded in a polyimide showed improvement in gas separation efficiency of $\mathrm{CO}_{2} / \mathrm{CH}_{4}$ but was unable to overcome the Robeson upper bound (the upper limit of Robeson plot that measures permeability against selectivity of gases in membranes) [7]. In another study, the MMM material with $\mathrm{UiO}-66(\mathrm{Zr})-(\mathrm{COOH})_{2}$ tested for the selective permeability of $\mathrm{CO}_{2}$ has proven to surpass the Robeson's upper bound from two strategic mixtures $\left(\mathrm{CO}_{2}: \mathrm{CH}_{4}=10: 90\right)$ and 
$\left(\mathrm{CO}_{2}: \mathrm{N}_{2}=15: 85\right)$ under the conditions 1.0 bar and $303 \mathrm{~K}$, as shown in Figure 1-1. The selective permeability of $\mathrm{CO}_{2}$ of this material was higher than that of both MOF and polymer matrix alone.
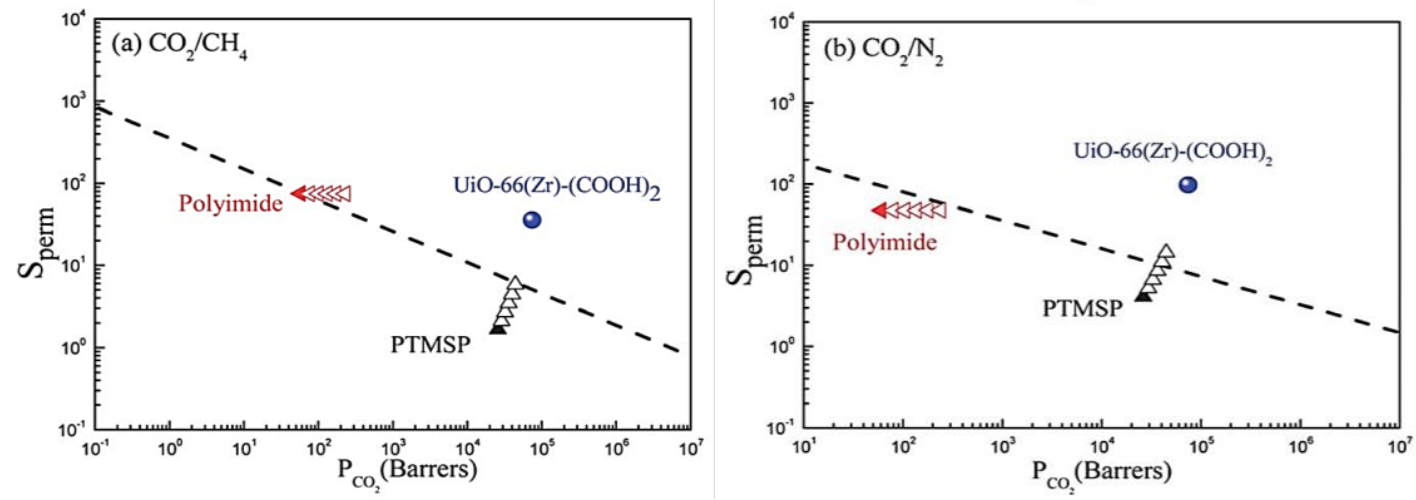

Figure 1-1: Computational performance of mixed matrix membranes in selective permeation of $\mathrm{CO}_{2}$ in a mixture of $\mathrm{CO}_{2} / \mathrm{CH}_{4}$ and $\mathrm{CO}_{2} / \mathrm{N}_{2}$ [7].

As the MOFs bind to the polymer matrix with the chemical interaction of active sites, the designing of MOFs functionality and features becomes an important aspect in designing these MMMs. Designing these membranes is a straightforward procedure but also requires a strategic exploration of its inherent characteristics for successful application. These features are readily provided by UiO-66 because of its particle size ranging from 100-500 $\mu \mathrm{m}$ that ensures easy incorporation within the polymer matrix, facile tunability of pores via solvothermal synthesis, and unsurpassed chemical and mechanical stability in harsh membrane production settings [7]. Therefore, it is necessary that more efforts should be directed in determining the factors that affect the properties and formulation of MOFs that would enable one to develop a superior MOF with outstanding features. Moreover, it is worthwhile to gain insight of the formulation of MOFs that will help in rational prediction of structure through precise control over its design. 


\section{$1.1 \quad$ Outline}

The purpose of this thesis was to study the effect of the change in process and compositional variables, such as the reaction time, temperature, concentration of reactants, stirring time, and solvent on the morphology, crystallinity, and surface area of Ui0-66 were comprehensively studied. After the introduction, Chapter 2 provides a thorough literature review of MOFs, the popular Zr-based MOFs and its current applications with specific focus on the Zr-based MOF - UiO-66 and its exceptional characteristics. A portion of this chapter is also dedicated to the popular MOF synthesis techniques used along with their associated pros and cons. Chapter 3 describes all the compounds, experimental set up and analytical tools used for the solvothermal synthesis and characterization of UiO-66. It also lists the raw materials and equipment used to study the factors in the synthesis of UiO-66 without cosolvent and with co-solvent. The last part of this section describes the theory behind various analytical techniques used in characterizing the resultant samples. Chapter 4 consists of 9 subsections which investigates the effect of altering the process and composition variables using the solvothermal synthesis on the structural properties of UiO-66. In addition, cosolvents (ethylene glycol, diethylene glycol, triethylene glycol) and their concentrations, and the role of acetic acid as a modulator on the morphology of UiO-66 synthesized with ethylene glycol as a co-solvent were also experimented. Next, Chapter 5 reiterates the conclusions drawn from each section of chapter 4 . The last chapter, Chapter $\mathbf{6}$, concludes the thesis with recommendations and impending work of the study. 


\section{CHAPTER TWO \\ LITERATURE REVIEW}

The aim of this chapter is to provide a brief review of metal organic frameworks with an introduction on its development overtime. An overview of significant Zr-based MOFs will also be followed by the most significant Zr-based MOF, UiO-66, its crystal structure and the factors that contribute to its outstanding stability. The various synthesis routes available in MOF production will be describes and narrowed to the conventional solvothermal synthesis route of UiO-66. Based on this, the factors that affect the morphology of UiO-66 will be analyzed with finally highlighting the prominent applications of UiO-66.

\section{$2.1 \quad$ Metal-organic Frameworks}

Metal-organic frameworks, also known as MOFs, were first invented in 1989 and have since emerged as alternative functional microporous materials, similar to zeolites [8]. They are highly crystalline, inorganic-organic hybrid, $n$-dimensional scaffolds, containing central metal ions or clusters and organic linkers joined together via covalent bonding [9]. These hybrid metal ions-organic linkers building units conjoin to form network solids containing pores of different sizes depending on the length of the linkers.

MOFs can be viewed as an extension of the zeolite family that offer lower thermal stabilities than zeolites but are rather more flexible in terms of framework rigidity and functionality [9]. Their pores allow guest molecules to be absorbed within the framework and expel them upon the application of external stimulus [9]. Due to their framework flexibility and the van der Waals force between guest molecules and frameworks, the pores deform and restore during the adsorption-desorption cycle. This unique phenomenon is exclusive to MOFs and is referred to as the gate-opening or breathing effect [9].

Despite the wide range of tunable characteristics, MOFs face some critical challenges in terms of thermal and mechanical stability that limit their use in industrial applications. These weaknesses arise from the lability of covalent bonds at high temperatures and is a prominent 
disadvantage that hinders its development [4]. In order to overcome these flaws, efforts are directed towards modifying the synthesis process using sophisticated post-synthesis modification techniques [10], [11] which increase its stability and efficiency. However, the improved qualities are achieved at the expense of their surface areas and functionality, besides being time consuming, and energy and labor intensive [4].

Efforts have been put into developing superior MOFs solely by using different synthesis mixtures. Some prominent MOFs in this field include Zr-MOFs, specifically the isoreticular series of UiO-66, MILs, and ZIFs [1]; some of these structures are presented in Figure 2.1-1. For instance, ZIF-8, a zeolitic imidazolate framework, is a tetrahedral coordinated transition metal joined by imidazolate linkers as T-Im-T [12] ( $\mathrm{T}$ =tetrahedrally coordinated metal ion, such as Zn or Co (blue tetrahedral units); Im=imidazolate linker (grey organic molecules connecting blue tetrahedral units)) that resembles the Si-O-Si bond angle usually found in zeolites [13]. It is the most explored MOF due to its substantial physiochemical properties [13] but requires further post synthesis modifications to improve its selectivity in adsorption and separation [14].

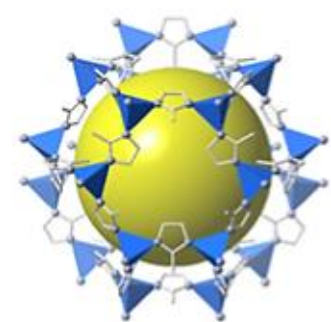

ZIF-8

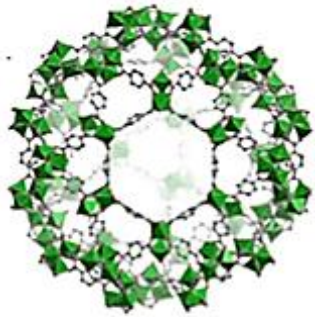

MIL-101

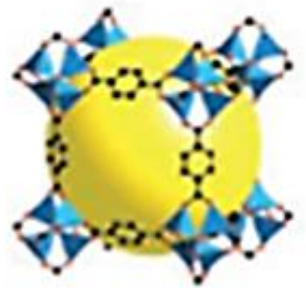

MOF-5

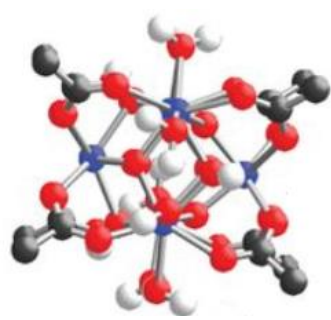

NU-1000

Figure 2.1-1: Molecular structure of various MOFs [2], [4], [15], [16]

MIL-101, discovered by Ferey et al. [13], utilizes its large surface area in gas absorption and catalysis due to it moisture and chemical resistant properties. It contains $\mathrm{Cr}$ metal atoms connected by $\mathrm{H}_{2} \mathrm{BDC}$ linkers with a BET surface area of $4000 \mathrm{~m}^{2} / \mathrm{g}$ [12]. However, the large surface is difficult to obtain due to high framework impurity [13]. Another prominent framework in absorption and catalysis is that of MOF-5 by Yaghi et al. [2]. 
Nevertheless, the weak hydrothermal stability of MOF-5 led to investigating the properties of HKUST-1 with higher thermal stability and relatively easier synthesis [13]. Additionally, with a coordination mode similar to UiO-66, NU-1000 is ideal for heterogeneous catalysis and gas separation, and can be easily scaled up for commercial purposes [17]. The framework consists of twelve carboxylate ligands without any terminal hydroxyl or aqua ligand [10]. Despite the large surface area of $2315 \mathrm{~m}^{2} / \mathrm{g}$, NU-1000 experiences structural collapse under thermal activation, calling for other mandatory post-synthesis modifications to circumvent this downside [10], [18]. Therefore, regardless of the large surface areas and plausible guest molecule interaction, MOFs usually encounter challenges such as thermal degradation or complete structural collapse at high working temperatures, which impair its fundamental properties, thereby defeating the purpose of the material. Ever since its discovery, numerous researchers have explored the several synthesis procedures to modify its properties, functionality and applications [4].

\subsection{Applications}

The applicability of zirconium MOFs is extended to a wide range of fields due to the morphological differences in their pore sizes, surface areas, thermal and structural stability, and mechanical strength. These remarkable properties have led to the potential industrial utilization of Zr-MOF in the areas of gas storage, molecule adsorption and separation, drug delivery, and catalysis.

\subsubsection{Gas Storage}

The alarming pollutant gas levels in the environment call for an urgent sustainable gas disposal method. Since zirconium MOFs exhibit large surface areas, and permanent porosity, they have been extensively reviewed as a promising candidate in the field of gas storage. Due to the high tunability in pore sizes, these Zr-MOFs can be adjusted to filter different types of gases, depending on their linker length and availability of surface area.

Researchers have data mined and characterized 38,000 MOFs by screening the Cambridge Structure Database that showed numerous MOFs are yet to be studied for gas storage properties [19]. A simulated study employing data mining and automated structure analysis 
screened about 22,700 compounds out of which 4,000 MOFs with surface areas and porosities appropriate for hydrogen gas uptake were identified [19]. The assumption of complete removal of solvent/guest molecules, i.e., complete activation, generated large surface areas compared to experimentally obtained surface areas; therefore, a realistic figure would filter out more compounds due to incomplete solvent removal, rendering them inapplicable. Therefore, it is evident that rigorous research is required to identify and apply suitable MOF in the gas storage applications. However, a handful of researches demonstrate the utilization of zirconium-based MOFs in gas storage.

The insufficient knowledge of the environmental impact of hydrogen gas urged researchers to study the environmental effect of switching from fossil fuels to hydrogen fuel cells [20]. According to a recent publication from Jacobson's group at Stanford University, the sources of environmental hydrogen gas are numerous with predominant sources being emissions from vehicles and electric power plants. The hydrogen gas causes the formation of polar stratospheric clouds and stratospheric aerosols, which in turn induces reduction of ozone gases, thereby leading to global warming [21]. Therefore, the need to look out for a sustainable gas storage is instinctive.

Based on the different pore sizes and surface areas, the following section discusses the various zirconium MOFs applied to the storage of hydrogen gas. Table 2.2.1-1 represents the different zirconium-based MOFs assessed for their hydrogen uptake capacities. A close comparison between these experimental values shows that MOF-808 is the most suitable material for this application, due to its significant $\mathrm{H}_{2}$ gas uptake capacity at both low and high pressures. 
Table 2.2.1-1: Summary of hydrogen gas uptake capacities of Zr-based MOFs.

\begin{tabular}{|c|c|c|c|c|}
\hline Zr-MOF & Ligand/Linker & $\begin{array}{l}\text { BET surface } \\
\operatorname{area}\left(\mathrm{m}^{2} / \mathrm{g}\right)\end{array}$ & $\begin{array}{c}\text { H}_{2} \text { uptake capacity } \\
\text { (wt\%) }\end{array}$ & Ref \\
\hline UiO-66 & $\mathrm{BDC}^{2-}$ & 1434 & $\begin{array}{c}1.4(1 \mathrm{bar}, 77 \mathrm{~K}) \\
3.2(30 \mathrm{bar}, 77 \mathrm{~K}) \\
1.6(1 \mathrm{~atm}, 77 \mathrm{~K}) \\
4.2(6 \mathrm{MPa}, 77 \mathrm{~K})\end{array}$ & $\begin{array}{l}{[12]} \\
{[12]} \\
{[13]} \\
{[13]}\end{array}$ \\
\hline $\mathrm{UiO}-67$ & $\mathrm{BPDC}^{2-}$ & 2313.61 & $\begin{array}{c}6.08(30 \text { bar, } 77 \mathrm{~K}) \\
1.75(1 \text { bar, } 77 \mathrm{~K})\end{array}$ & [12] \\
\hline MOF-801 & FUM" ${ }^{2-}$ & 726.51 & $3(30$ bar, $77 \mathrm{~K})$ & {$[12]$} \\
\hline MOF-802 & $\mathrm{PZDC}^{2-}$ & 17.33 & $1.47(30$ bar, $77 \mathrm{~K})$ & {$[12]$} \\
\hline MOF-808 & $\mathrm{BTC}^{3-}$ & 2242.77 & $\begin{array}{c}2.79(1 \text { bar, } 77 \mathrm{~K}) \\
5.85(30 \mathrm{bar}, 77 \mathrm{~K})\end{array}$ & $\begin{array}{l}{[12]} \\
{[12]}\end{array}$ \\
\hline MOF-841 & MTB $^{4-}$ & 1403.77 & $2.52(1$ bar, $77 \mathrm{~K})$ & {$[12]$} \\
\hline
\end{tabular}

One of the promising sustainable applications offered by Zr-MOFs due to its large penetrable surface areas is adsorption. The restorability of the breathable pores demonstrates sustainable performance in molecular adsorption and separation applications.

Another major contributor to global warming is the substantial increase in carbon dioxide levels in the atmosphere [22]. The amount of $\mathrm{CO}_{2}$ constantly being on the rise not only adversely affects human life, but also endangers the ecological cycle. The $\mathrm{CO}_{2}$ uptake capacity reported for UiO-66 was 13.4 at 1 bar [23]. Since this value was significantly small for practical applications, it was further altered through post-synthetic functionalization to enhance their $\mathrm{CO}_{2}$ uptake capacities. The post-synthetic modification of $\mathrm{UiO66}-\mathrm{COOH}_{2}$ by introducing alkaline metals such as $\mathrm{Li}^{+}, \mathrm{Na}^{+}$, and $\mathrm{K}^{+}$showed enhanced carbon dioxide capture capacities with a $\mathrm{CO}_{2} / \mathrm{N}_{2}$ selectivity of 99.6 with UiO66-COONa, being the highest value reported for the binary flue gas [3]. A research published by Abid et al included the introduction of ammonium hydroxide to create textural change in UiO-66. Although this introduction reduced the surface area and porosity of UiO-66, the $\mathrm{CO}_{2}$ adsorption was improved [23]. The adsorption of $\mathrm{CO}_{2}$ was reported to be $7.8 \mathrm{mmol} / \mathrm{g}$ at $273 \mathrm{~K}, 988 \mathrm{KPa}$ [23]. Through another metalated exchange as a post-synthetic modification technique for UiO-66, the adsorption capacities of $\mathrm{UiO}-66-(\mathrm{COONa})_{2}$ and $\mathrm{UiO}-66-(\mathrm{COOLi})_{2}$ were experimented for $\mathrm{CO}_{2}$ [24]. The selectivity values for $\mathrm{CO}_{2} / \mathrm{N}_{2}$ was 33.3 and 26.6 for UiO-66-(COONa) 2 and UiO- 
66-(COOLi)2, respectively at 1 bar and $298 \mathrm{~K}$, with $\mathrm{CO}_{2}$ uptake capacities of $2.93 \mathrm{mmol} / \mathrm{g}$ and $3.44 \mathrm{mmol} / \mathrm{g}$, respectively at $1 \mathrm{bar}, 273 \mathrm{~K}$ [24]. All these examples imply a great potential of Zr-MOFs in $\mathrm{CO}_{2}$ capture application.

\subsubsection{Drug Delivery}

Several challenges need to be met in order to achieve the best material as a drug carrier. Among the various requirements for the finest carrier, it is essential that the material entraps majority portion of the drug, provides controlled release into the body fluids, resists structural degradation, allow surface modification, and be trackable through imaging techniques [25]. Evidently, most of these requirements are inherently exhibited by zirconium MOFs. In comparison to other MOFs, zirconium MOFs excel in this specific application due to their high chemical stability and large surface area. Moreover, since zirconium MOFs offer multivalent interaction sites, the enhanced host to guest interaction within the pores aids their performance as drug delivery vehicles [26].

Zirconium MOFs are inherently highly crystalline mesoporous materials with inherent chemical stability that offer heavy loading capacity along with protected and controlled drug delivery to the desired site [26]. However, the pores require to be designed based size of the adsorbate molecule [27]. In order to study the applicability of zirconium MOFs in drug delivery, numerous studies have been done in terms of drug encapsulation in physiologically simulated fluids [27], [28]. Accordingly, Horcaja et al. [29] demonstrated the influence of MOFs properties on drug encapsulation and delivery performance of two model drugs, namely, aspirin (AAS) and ibuprofen (IBU), using three different water-stable MOFs - MIL$100(\mathrm{Fe}), \mathrm{UiO}-66$, and MIL-127(Fe), which was carried out at $37^{\circ} \mathrm{C}$. It was found that UiO-66 ranked highest in the drug loading capacities for both IBU and AAS, as $92 \%$ and $75 \%$ of the total concentration of drug available in the solution, respectively, within the first $6 \mathrm{~h}$ despite having smaller cavities than its competitors. This was because of the octahedral and tetrahedral pores having functional groups attached to the benzene ring that facilitated weak interactions such as van der Waal forces, hydrogen bonding, and $\pi-\pi$ stacking. On the other hand, MIL-100 and MIL-127 had drug loading capacities of IBU and AAS as, 79\% and 72\%, 
and $35 \%$ and $13 \%$, respectively, at $24 \mathrm{~h}$. The low capacities were attributed to the reduction in pore accessibility due to host-guest interaction of ethanol molecules with the metal centers. In terms of the drug release activity, UiO-66 achieved 96\% and 46\% for AAS and IBU. These numbers were justified by the hydrophilicity/ hydrophobicity of the model drugs. UiO-66 being hydrophobic along with its concomitant specie, IBU, lowered the drug diffusion rate; while, AAS being dissimilar in nature diffused faster. MIL-100 showed higher drug release of up to $84 \%$ and $99 \%$ for IBU and AAS, respectively. This was because of the large pores and hydrophilic nature of the MOF which lead to faster diffusions. MIL-127, in contrast, demonstrated poor performance in drug release, with only $35.2 \%$ and $12.94 \%$ for IBU and AAS, respectively. Thus, considering both the cases and overall performance, UiO-66 displayed a good trade off between the drug encapsulation and drug release, being an ideal candidate for drug delivery application.

Another breakthrough in drug delivery application was studied by Filippousi et al. [27] in the delivery of $\varepsilon$-caprolactone as an anti-cancer drug. In this study, UiO-66 and UiO-67 were used as the carriers to adsorb two typical drugs, hydrophobic paclitaxel/taxol and hydrophilic cisplatin, and further encapsulated in $\varepsilon$-caprolactone [27]. It was observed that the loading of cisplatin and Taxol decreased the Langmuir surface area very slightly in contrast to the UiO-66 crystals before loading, from $840 \mathrm{~m}^{2} / \mathrm{g}$ to $672 \mathrm{~m}^{2} / \mathrm{g}$, with marginal decrease in pore volume, from $0.32 \mathrm{~cm}^{3} / \mathrm{g}$ to $0.25 \mathrm{~cm}^{3} / \mathrm{g}$ [27]. However, the loading was more for UiO-66 than UiO-67 despite being lower in surface area. Taxol being a larger molecule than cisplatin could not be readily adsorbed by UiO-66, but just fit in UiO-67 due to the missing linker defect [27]. On the other hand, cisplatin was easily adsorbed in UiO-67, but was unable to fit into the smaller pores of UiO-66. According to this observation, it was theorized that MOF should have either recurring linker defects to incorporate Taxol in UiO67 or recurring inorganic brick defects in UiO-66; or introducing missing linker defects in UiO-66 to incorporate cisplatin [27]. In conclusion, Taxol loaded MOF exhibited a high dissolution rate due to amorphization, while cisplatin loaded MOFs offered a constant disintegration rate [27]. Therefore, despite the negative changes in surface areas, both cisplatin loaded MOFs exhibited promising results as an anti-cancer drug delivery material. 
Similarly, Zhu et al. [30] experimented the function of UiO-66 as an alendronate (AL) drug carrier. The obvious reduction of BET surface area from $1136 \mathrm{~m}^{2} / \mathrm{g}$ to $45 \mathrm{~m}^{2} / \mathrm{g}$ due to AL loading was observed [30]. As a drug carrier, it was found that the $59 \%$ of the AL drug was released at $\mathrm{pH}$ of 5.5 , while it was $42.7 \%$ at $\mathrm{pH}$ of 7.4 , with release rates of $0.0104 \mathrm{~g}_{\mathrm{AL}} / \mathrm{g}_{\mathrm{MOF}} \cdot \mathrm{h}$ and $0.0075 \mathrm{~g}_{\mathrm{AL}} / \mathrm{g}_{\mathrm{MOF}} \cdot \mathrm{h}$, respectively [30]. The amount released was also found to increase overtime, being $88.1 \%$ and $76 \%$, by $108 \mathrm{~h}$ for $\mathrm{pH}$ of 5.5 and 7.4 , respectively. The high adsorption amount for the acidic medium was attributed to heightened protonation of phosphates in acidic medium that weakens the bonds with the $\mathrm{Zr}-\mathrm{O}$ moieties [30]. Furthermore, the therapeutic effect of AL carriers was demonstrated by in vitro cytotoxicity measurements in cancer cells. It was noted that-more cancer cell deaths were observed in AL loaded UiO-66 than free AL, for a 48h incubation period; while the cytotoxicity of cancer cells was more in free AL than AL carrier for a $24 \mathrm{~h}$ incubation period [30]. Hence, this experiment makes UiO-66 an effective anti-cancer drug delivery vehicle.

Hence, the apparent ease in the production of zirconium-MOFs along with high ordered structure sense of orientation, suggests high potential in application of drug delivery applications [25]-[28].

\subsubsection{Catalysis}

A comparison of the catalytic activity of MOF's precursors and synthesized MOF as catalysts, by Arrozi et al. [31] implied that the morphology of the MOF plays a significant role in enhancing the catalytic activity as opposed to its precursors. The reason being that the addition of linkers with high electron withdrawing character in the reaction decreased the electron density at the zirconium cluster compared to its precursor [31]. The effect resulted in high Lewis acidity that increased the reactivity of the substrate [31]. Moreover, it was highlighted that although the pore size and Lewis activity of UiO-67 was higher than that of Ui0-66, the catalytic activity of Ui0-66 was higher (91\%) than Ui0-67 (81\%) in the acetalization of benzaldehydes with alcohols [31]. This was attributed to the fact that UiO67 experienced pore blockage due to solvent entrapment, while the $\mathrm{Zr}$ ion in the cluster of UiO-66 was not fully coordinated, leading to unsaturated metal sites with more active sites 
and large accessible surface area. Therefore, it was pointed out that substrate accessibility is a governing factor in catalysis rather than the Lewis activity of the catalyst itself [31].

Control experiments, by Long et al. [32], confirmed the functionality of amine functionalized UiO-66 compared to its precursors UiO-66 and $\mathrm{ZrO}_{2}$. The photocatalytic application of amine functionalized $\mathrm{Zr}-\mathrm{MOF}$ in the aerobic oxygenation of alcohols, olefins and cyclic alkanes suggested that the activation of reacting substrates and molecular oxygen was key in defining the efficiency of the functionalized catalyst [32]. In this experiment, the organic reactive substrates were successfully oxidized by a mechanism of photo generated electron transfer utilizing UiO-66- $\mathrm{NH}_{2}$ [32]. The final product was then formed through a reaction of substrates with the carbonium ions [32]. In conclusion, this experiment yielded promising results of the amine functionalized UiO-66 as a photocatalyst.

As mentioned earlier, UiO-66 may not be as favorable as a functionalized sample of UiO-66 [32]. Therefore, researchers Zhou et al. [33] experimented the effect of linker defects on the catalytic activity of UiO-66 by adjusting the $\mathrm{Zr}$ :BDC ratio and the reaction temperature on transesterification of tributyin and soybean oil with methanol (a model reaction for biodiesel synthesis). It was observed that the UiO-66 with the most linker defects (UiO-66-100-1) resulted in highest conversion of $99.3 \%$ after $5 \mathrm{~h}$, while the UiO-66 with the less linker defects (UiO-66-220-2) resulted in lowest conversion of only 38.8\%. The results agreed with the theoretical concept that more acid sites and higher acid strength lead to increased catalytic activity [33]. Moreover, the recyclability of UiO-66-100-1 yielded a 95\% conversion after the $4^{\text {th }}$ run, indicating a reduction in surface area. Therefore, it was concluded that increasing linker defects enhanced the catalytic activity of the MOF [33].

Another example of the effect of functionalization of Zr-MOF affecting the catalytic activity was depicted in the epoxide ring opening of styrene oxide by methanol, using UiO-66-X as catalysts, where $\mathrm{X}=\mathrm{H}, \mathrm{NH}_{2}, \mathrm{NO}_{2}, \mathrm{Br}, \mathrm{Cl}[34]$. In this study, the catalytic activity depended on several factors such as the effect of functional group on Lewis acid sites, steric hindrance, position of functional group within the crystal lattice, density of lattice defects, and presence of sites activating the nucleophile [34]. It was found that the catalytic activity was the highest for UiO-66-Br; however, a particular reason could not be drawn for this behavior. With 
regard to the recyclability, it was observed that UiO-66-Br experienced deactivation over time due to product poisoning [34].

A research by Vermoortele et al. [35] laid the emphasis on synthetic modulation of UiO-66 to explore its full potential as a Lewis acid catalyst. It was observed that a modulated approach by using trifluoroacetic acid (TFA) as a modulator and hydrochloric acid ( $\mathrm{HCl})$ as a crystallizing agent yielded far better conversion than using TFA alone. Adding 20 equivalents of TFA in the synthesis precursor produced a UiO-66 showing 75\% citronellal conversion after $10 \mathrm{~h}$ of reaction time, which is much higher than that of UiO- 66 without TFA (34\%). Furthermore, the addition of $\mathrm{HCl}$ as a crystallizing agent to the synthesis precursor with TFA resulted in a UiO-66 showing $40 \%$ citronellal conversion at $1 \mathrm{~h}$ of reaction time (UiO-66-10 $\mathrm{HCl}$ ), which is higher than that of TFA included UiO-66 (21\%, UiO-66-10). In this case, hydrochloric acid promoted two essential functions in the reaction; first, the hydrolysis of $\mathrm{ZrCl}_{4}$, and second, the deprotonation of carboxylic acids [36]. The first function ensures more availability of central zirconium atom, the basis for cluster formation. While the TFA, being a stronger acid with a weaker conjugate base, serves the second purpose by competing with carboxylic acid ligand bonding within the cluster [36]. Therefore, the TFA as a modulator replaces the terephthalate linker with trifluoroacetate being incorporated in the hexanuclear cluster and the crystal boundaries [35]. To gain a broader perspective, $\mathrm{HCl}$ aids in the dissociation of BDC from zirconium metal ion by altering the intrinsically high association that balance the equilibrium [35]. Its presence also favors the development of $\mathrm{Zr}$ acetate complexes rather than its alternate thermodynamically favoured Zr-BDC complexes [35]. This activity ensures successful integration of trifluoroacetate ions in the crystal structure [35], wherein upon activation, this position of missing linkers in the crystal structure form coordinatively unsaturated Lewis acid sites [35] that increase the catalytic activity of the zirconium MOF. Subsequently, the crystal size and surface area were observed to be higher than those of as-synthesized UiO-66 [36]. However, it should be noted that the incorporation of TFA within the framework was merely due to the addition of $\mathrm{HCl}$, as TFA alone was not able to enhance the catalytic activity. It is noteworthy that $\mathrm{HCl}$ itself does not lead to the formal of additional active sites, but rather aids in the creation of active metal sites for TFA, and is therefore, ineffective in a non-modulated synthetic approach [35]. 


\section{$2.3 \quad$ Ui0-66 (Universitetet i Oslo)}

Among the zirconium-based MOFs, UiO-66 remains the most researched MOF since it strikes a perfect balance of the extraordinary thermal and chemical stability [40]-[42] which makes it an ideal candidate in the industrial applications such as catalysis, gas separation, $\mathrm{CO}_{2}$ storage, and drug delivery.

UiO-66 (Universitetet i Oslo), first developed by Professor Karl Petter Lillerud of the Department of Chemistry at the University of Oslo in 2008, is a zirconium-based MOF whose framework structure comprises of $\mathrm{Zr}_{6} \mathrm{O}_{8}$ clusters separated by BDC or 1,4-benzodicarboxylic acid linkers [36]-[38]. Zr-based MOFs have developed in its isorecticular series, namely, UiO67 and UiO-68, by selecting organic ligands of varying lengths. With a few exceptional variations, titanium (Ti) and hafnium (Hf) based MOFs from the Group IV elements in the periodic table were also discovered to be structurally identical to zirconium MOFs [1], [39]. Similarly, cerium (Ce), uranium (U), and thorium (Th) MOFs have also been in the process of development for attainting stable morphologies [1].

As per the focus of this project, this section will remain limited to the discussion of the $\mathrm{Zr}$ based MOFs. Table 2.3-1 lists the significant Zr-based MOFs with exceptionally large surface areas and their corresponding topological details. As evident, increase in the linker length simultaneously increases their pore size resulting in large surface area. However, the increasing surface area reduce its mechanical stability as these properties are inversely proportional [40]. 
Table 2.3-1: Remarkable Zr-based MOFs with exceptional surface areas [4].

\begin{tabular}{|c|c|c|c|c|}
\hline MOF & Linker/Ligand & Zr-cluster & Topology & $\begin{array}{l}\text { BET surface } \\
\text { area }\left(\mathrm{m}^{2} / \mathrm{g}\right)\end{array}$ \\
\hline UiO-66 & $\mathrm{BDC}^{2-}$ & $\begin{array}{c}\operatorname{Zr}_{6}\left(\mu_{3}-O\right)_{4}\left(\mu_{3}-\right. \\
O H)_{4}\end{array}$ & $\begin{array}{l}\text { fcu, } 12- \\
\text { coordinated }\end{array}$ & 1187 \\
\hline UiO-67 & $\mathrm{BPDC}^{2-}$ & $\begin{array}{c}\operatorname{Zr}_{6}\left(\mu_{3}-0\right)_{4}\left(\mu_{3}-\right. \\
\mathrm{OH})_{4}\end{array}$ & $\begin{array}{c}\text { fcu, } 12- \\
\text { coordinated }\end{array}$ & 3000 \\
\hline UiO-68 & TPDC $^{2-}$ & $\begin{array}{c}\operatorname{Zr}_{6}\left(\mu_{3}-0\right)_{4}\left(\mu_{3}-\right. \\
\mathrm{OH})_{4}\end{array}$ & $\begin{array}{c}\text { fcu, } 12- \\
\text { coordinated }\end{array}$ & 4170 \\
\hline $\begin{array}{l}\text { NU- } \\
1000\end{array}$ & TBAPy- & $\mathrm{Zr}_{6}\left(\mu_{3}-\mathrm{OH}\right)_{8}$ & $\begin{array}{l}\text { csq, }(4,8)- \\
\text { coordinated }\end{array}$ & 2320 \\
\hline $\begin{array}{l}\text { NU- } \\
1100\end{array}$ & PTBA $^{4-}$ & $\begin{array}{c}\mathrm{Zr}_{6}\left(\mu_{3}-0\right)_{4}\left(\mu_{3}-\right. \\
\mathrm{OH})_{4}\end{array}$ & $\begin{array}{l}\text { ftw, }(4,12)- \\
\text { coordinated }\end{array}$ & 4020 \\
\hline
\end{tabular}




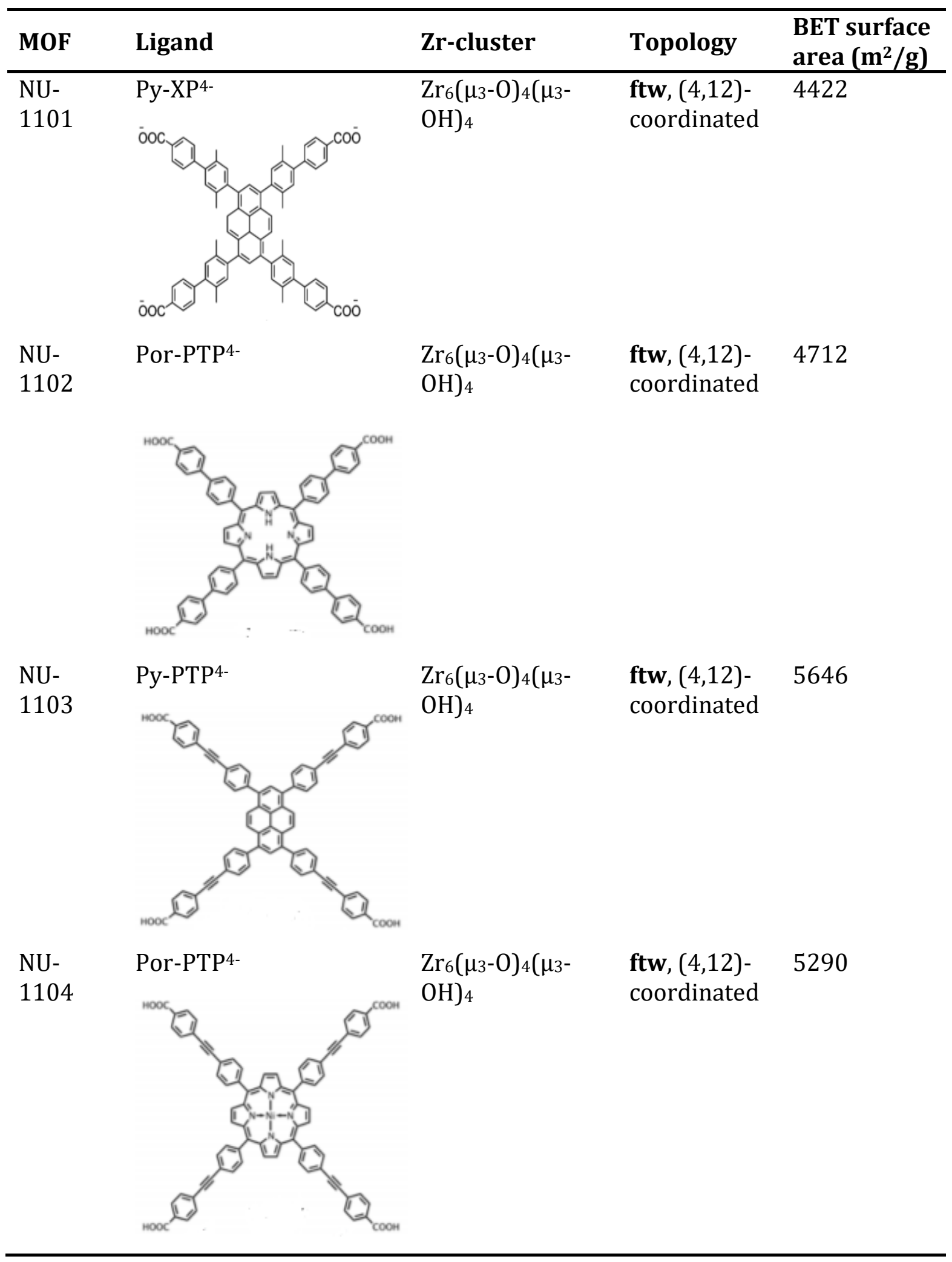




\subsubsection{Structural Description of Ui0-66}

The intricate microporous structure of UiO-66 with a Langmuir surface area of $1187 \mathrm{~m}^{2} / \mathrm{g}$ [37] comprises of hexanuclear $\left(\mathrm{Zr}_{6} \mathrm{O}_{4}(\mathrm{OH})_{4}\right)$ metal clusters (Figure 2.3.1-1b) known as the secondary building units (SBUs). SBUs/clusters are an integral part of any MOF and referred to as molecular complexes formed by covalent metal-ligand bonds. The $\mathrm{Zr}$ clusters are separated by (BDC) or 1,4-benzodicarboxylic acid linkers that form a highly packed face centered cubic (fcu) structure [37], [36], [38]. Each lattice point in the unit cell is a $\left(\mathrm{Zr}_{6} \mathrm{O}_{4}(\mathrm{OH})_{4}\left(\mathrm{CO}_{2}\right)_{12}\right)$ cluster, that consists of 8-coordinated zirconium metal ions, with each zirconium metal connected to 8 oxygen atoms in a square-antiprismatic geometry [37]. The geometry is further bridged by alternating $\mu_{3}-\mathrm{O}$ and $\mu_{3}-\mathrm{OH}$ bridges around one triangular face, to 4 oxygen atoms from the carboxylate linker around the other triangular face [37], [1], [36]. The octahedral (Figure 2.3.1-1b) and tetrahedral cages (Figure 2.3.1-1a) have a diameter of $11 \AA ̊$ and $8 \AA$, respectively in a 1:2 octahedral:tetrahedral ratio [35], as shown in Figure 2.3.1-1c, which come together to form a cubic unit. The pores are large enough to fit a fully methylated benzene molecule [40]. The SBU is connected to 12 other SBUs via carboxylate linkers, setting a highest reported coordination number of 12 [1], [38].
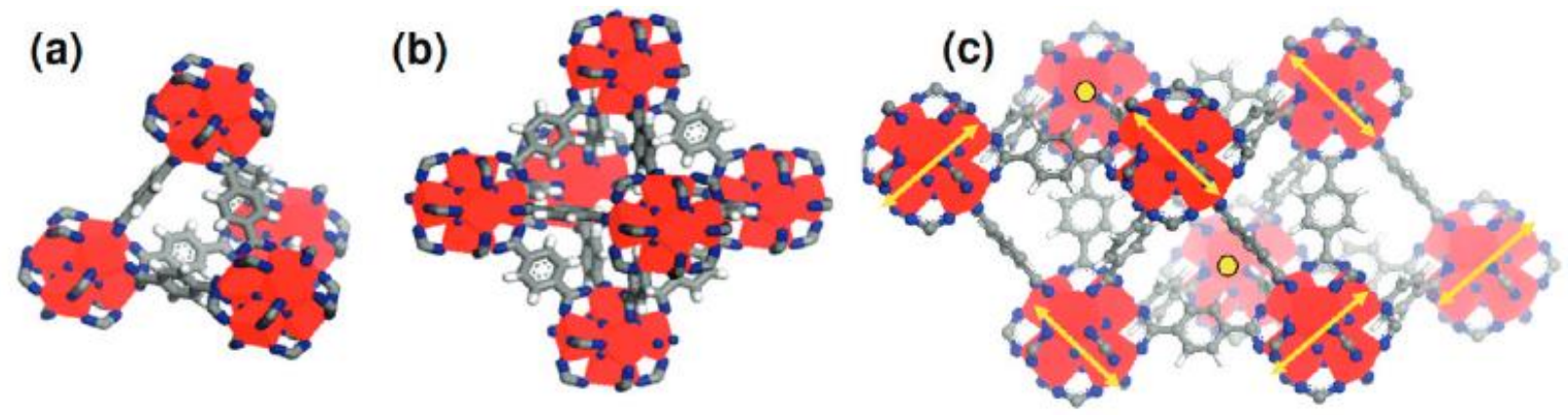

Figure 2.3.1-1: Structure of UiO-66 MOF. Colors red, grey, blue and white represent zirconium, carbon, oxygen, and hydrogen atoms, respectively. The grey, blue and white structures are the BDC linkers connecting the SBUs (red, grey, blue). Illustrated are (a) Tetrahedral cage. (b) Octahedral cage. (c) $8 \mathrm{Zr}_{6} \mathrm{O}_{4}(\mathrm{OH})_{4}$ bricks formed by combination of one octahedral cage and two adjacent tetrahedral cages forming a cubic unit with yellow arrows 
representing the squeezing direction that the $\mathrm{Zr}_{6} \mathrm{O}_{6}$ octahedron undergoes after the dehydroxylation process. Yellow circles point perpendicular to the page [41].

\subsubsection{Mechanical Strength and Chemical Stability of UiO-66}

UiO-66 is one of the most investigated MOFs because of its unprecedented physiognomic properties such as exceptional thermal and chemical stability, high porosity and resistance to adverse environment which opens doors to applications in gas separation, catalytic reactions, drug delivery and florescence sensing among others [4]. Its central metal atom being zirconium has a high affinity towards oxygen ligands resulting in a directional compact structure [40]. The plasticity of this material is highly limited due to the directional bonding between organic ligands and metal clusters in the organic-inorganic framework. The feature yields high elastic modulus ( $\mathrm{G}_{\min }=13.7 \mathrm{GPa}$ ) which is comparable to that of zeolites ( $20 \mathrm{GPa}$ ) and shows high durability against shear stress [40]. Additionally, the crystallinity remains unaltered upon application of $10,000 \mathrm{~kg} / \mathrm{cm}^{2}$ of external pressure [40]. Therefore, besides being highly porous, UiO-66 exhibits exceptional mechanical strength, although porosity and mechanical strength are inversely proportional [42]. Recent experiments also revealed high integral stability through the XRDs of the desolvated (free of residual solvent) UiO-66 with nearly unaltered patterns after being heated at $300^{\circ} \mathrm{C}$ for 1 hour [40], [32]. This material also shows resistance to water and other harsh organic solvents, such as DMF, benzene, acetone and acidic aqueous solutions [4], [41]. This stability is due to the strong coordination bond with the hard acid ( $\mathrm{Zr}^{4+}$ ions) and hard base (carboxylate linkers) [5].

These noteworthy qualities arise from its great bonding capabilities that can be understood by examining the structures at molecular level through comprehensive characterization methods. In the light of coordination chemistry, there are various factors that contribute to the high chemical stability of MOFs. These include the ionic radius and the reduction potential of metal atoms, metal-ligand coordination and bond strength, oxidation states, and $\mathrm{p} K_{\mathrm{a}}$ of linkers [5]. These factors are primarily linked to the valency of the central metal atom, forming the core for its inherent stability and determines the strength of the overall molecule. In case of UiO-66, the inherent stability comes from its high valency central metal 
ion. Zirconium, being a group IV element, has a high charge to radius ratio $(\mathrm{Z} / \mathrm{R})$ that manifests a strong ionic attraction with its bonding species [36]. It also possesses a +4oxidation state compared to other low oxidation states of MOFs [5]. The resulting high charge density of this element creates a strong force of attraction between the $\mathrm{Zr}^{4+}$ metal ions and the incoming carboxylic acid linkers, thus, creating a strong metal-ligand bond [4], [36], [40]. Moreover, the metal-ligand bond concept is in line with the Pearson's hard acid and base interaction concept where bond polarization results in stronger coordination bond [5]. The second rationale behind its outstanding chemical stability owes to the high nuclearity of the growing SBUs [36]. The SBUs of UiO-66 show high cluster-to-cluster and cluster-linker connectivity due to the strong ionic attraction between zirconium atoms [1], [36]. Besides, its high coordination number makes it resistant to nucleophilic attacks at ligand bonding sites and less likely to be substituted than metal centers with lower coordination number [36].

In terms of the characterization techniques, the combined efforts of Lamberti, Lillerud and their colleagues [41] have produced a thorough analysis of the structural, vibrational and electronic properties of UiO-66 by using both sophisticated theoretical and experimental methods such as PXRD, FTIR, and TGA that testify its thermal and chemical stabilities. A comparison of the PXRD patterns of UiO-66 with varying activation temperature, i.e., the temperature at which the so entrapped residual solvent molecules are removed from the pores of UiO-66, in air (Figure 2.3.2-1a), revealed that the Zr-oxo-hydroxo cluster in UiO-66 framework demonstrates high chemical stability up to $375^{\circ} \mathrm{C}$ in air with appreciable framework collapse only beyond this temperature [41]. The simultaneous TGA/MS experiments further attested to the PXRD results, providing detailed guest molecule desorption temperatures, and composition of structure upon complete breakdown. The TGA of UiO-66 shows weight loss in three consecutive stages as shown Figure 2.3.2-1b - (1) removal of physiosorbed water molecules (2) removal of solvent (3) the conversion of SBU $\mathrm{Zr}_{6} \mathrm{O}_{4}(\mathrm{OH})_{4}$ into $\mathrm{Zr}_{6} \mathrm{O}_{6}$. The first shoulder corresponding to $100^{\circ} \mathrm{C}$ was the water molecules followed by solvent molecules trapped within the pores leaving a material of the chemical formula $\mathrm{ZrO}\left(\mathrm{CO}_{2}\right)_{2}\left(\mathrm{C}_{6} \mathrm{H}_{4}\right)$. The next drop marked the degradation of $\mathrm{Zr}$-oxo-hydroxo cluster which involves removal of two $\mu_{3}-\mathrm{OH}$ groups with hydrogen from the $\mu_{3}-\mathrm{O}$ group and is 
completely dehydroxylated at $300^{\circ} \mathrm{C}$ [37]. Interestingly, after the dehydroxylation process, the $\mathrm{Zr}_{6} \mathrm{O}_{6}$ cluster changes its coordination number of zirconium atom from 12 to 7 or 8 , keeping an overall charge balance by bringing the structure back to its original position. This is done with minor changes in the bidentate (two donor groups in a single ligand) edge carboxylate groups to monodentate (one donor groups in a single ligand) with little effect on the core cluster [37]. The lattice decomposes fully starting at $500^{\circ} \mathrm{C}$ to $550^{\circ} \mathrm{C}$ with benzene in the gas phase, indicating the metal-ligand bond to be the strongest link in the framework [37]. The remnants of TGA analysis confirm $\mathrm{ZrO}_{2}$ as the product with poor crystallinity [37]. The molecules lost during the TGA process were confirmed by MS experiments and are represented in Figure 2.3.2-1c, d, e [37].
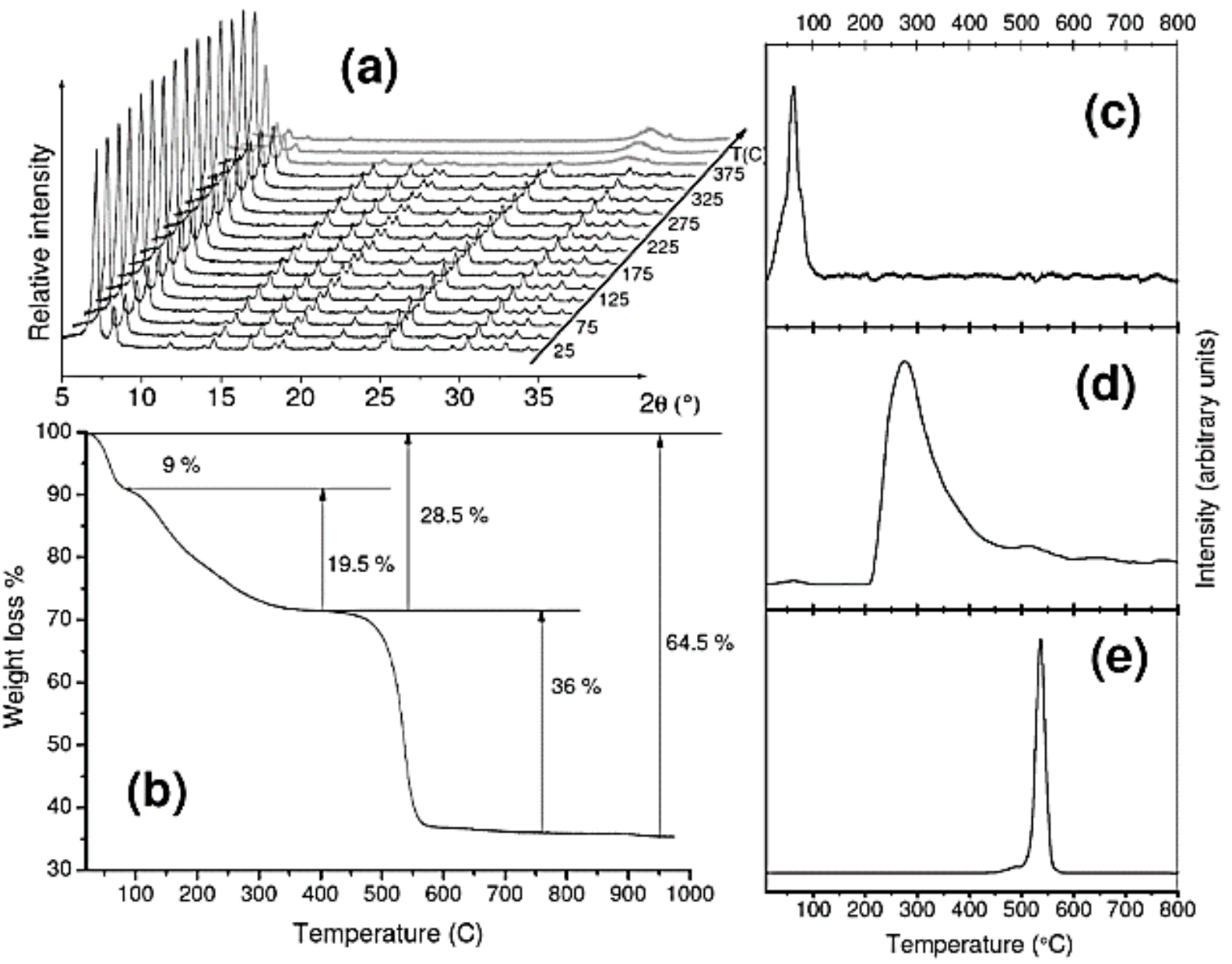

Figure 2.3.2-1: Thermal stability of UiO-66: a) PXRD profiles of UiO-66 with increasing activation temperature in air b) TGA curve of UiO-66 in air c), d), and e) MS results of water, DMF, benzene, respectively [41]. 
As shown in Figure 2.3.2-2, UiO-66 was also tested for its stability in water and harsh solvents such as DMF, benzene, acetone, ethanol, basic solution $(\mathrm{NaOH})$, acidic solution $(\mathrm{HCl})$, and water which showed that UiO-66 held its crystallinity for the most part. The Si is the internal standard added to qualitatively analyze the change in peak positions for UiO-66 samples in the named solvents. As observed, the PXRD patterns of UiO-66 in DMF, benzene, acetone, water, ethanol and $\mathrm{HCl}$ overlapped with the as-synthesized sample of UiO-66, but slightly shifted to lower angle when suspended in $\mathrm{NaOH}$ solution. This commendable stability in solutions can be backed up with the natural bond orbital theory (NBO).
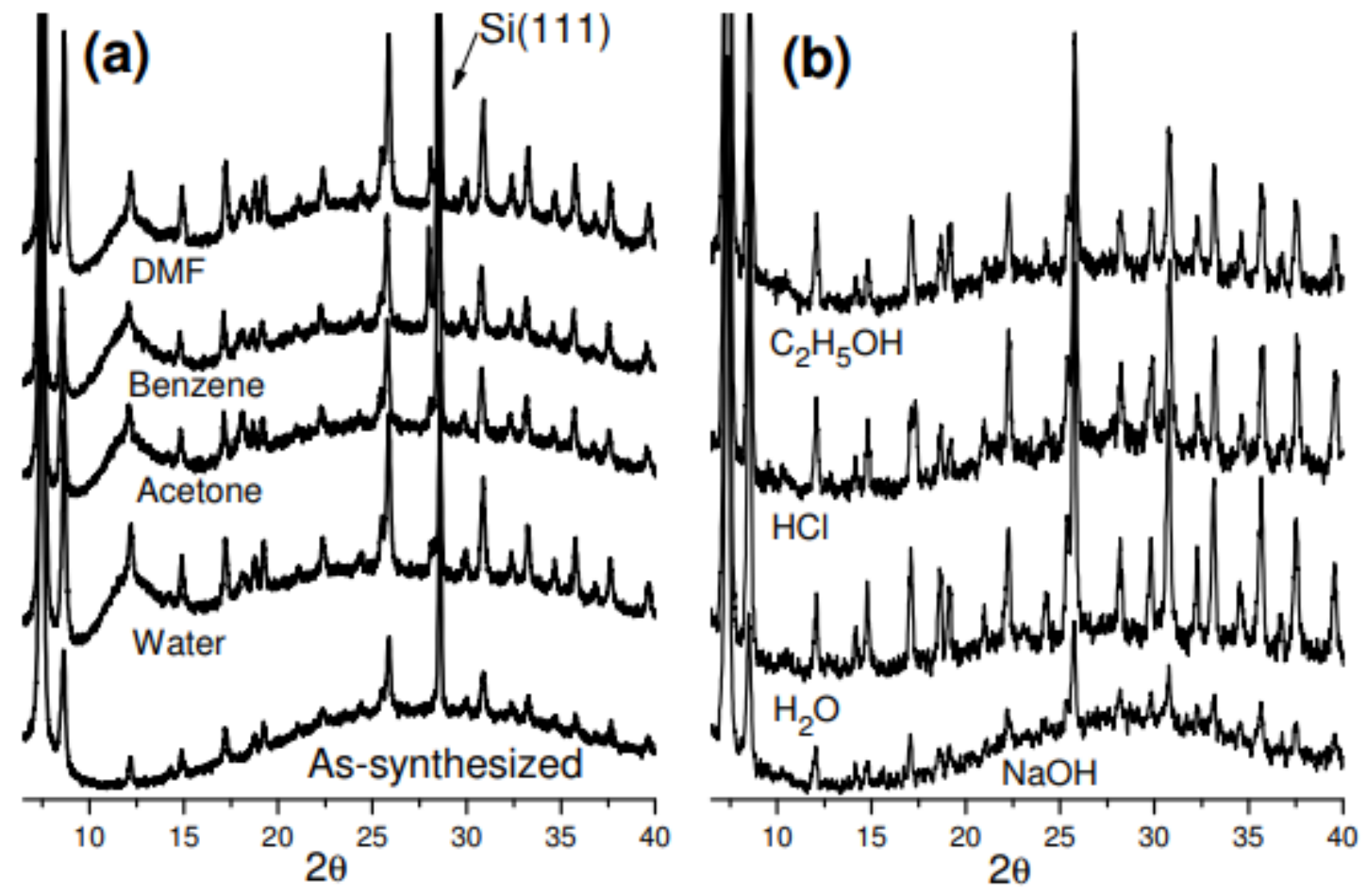

Figure 2.3.2-2: Structural stability of UiO-66: a) PXRD profiles of desolvated UiO-66 in the indicated solvents at room temperature in various stirred suspension solutions for $24 \mathrm{~h}, \mathrm{~b}$ ) PXRD profiles of desolvated UiO-66 in the indicated solvents at room temperature in various stirred suspension solution for 2 hours. The arrow in part (a) is the Si(111) reflection at $2 \theta$ $=28.4^{\circ}$ used for calibration. $\lambda=1.5406 \AA$ [41]. 
The NBO theory is based on the electron density of the molecular bonds that individually represent 1-2 localized orbital interactions within or between atoms. The electron density is represented by the Schrödinger equation (2-1),

$$
\mathrm{H} \Psi=\mathrm{E} \Psi
$$

where $\Psi$ is a wave function of the electron, $\Psi=\Psi_{1}\left(\rho_{1}\right)+\Psi_{2}\left(\rho_{2}\right)+\ldots$ where each bond orbital is an element of the overall electron density function represented by $\rho$, while the function $\Psi$ is an integer value between 0 and 2 included. The value 2 stands for the most bonding orbital while 0 stands for the anti-bonding orbital. The charge of 0 atoms from the carboxylate linker is -0.74; while the charge of 0 atoms from the $\mathrm{OH}^{-}$atoms in basic solution is -1.403 , which is comparatively smaller [4]. The high negative charge implies that 0 from the $\mathrm{OH}^{-}$ atoms can readily bond with zirconium atoms [4]. Hence, the bonds begin to decompose in basic solutions. This capability of UiO-66 to retain its structure surpasses other comparable MOFs as promising structures with diverse applicability.

\subsection{Synthesis Methods of Metal-organic Frameworks}

The need for flexible, robust and promising structure had led to the discovery of new class of microporous materials. This section presents a detailed discussion of the various available methods for MOF synthesis along with the pros and cons of adopting each technique. The production of new MOFs has seen an exponential growth over the last two decades (Figure 2.4-1). Its progress has been significant due to intriguing structures and aforementioned applications. These structures can be perceived as an extension to the field of zeolites while offering nearly equal robustness and porosities, but with breathable and flexible pores. For the most part, its physiochemical properties have led researchers to expanding and developing this field. 


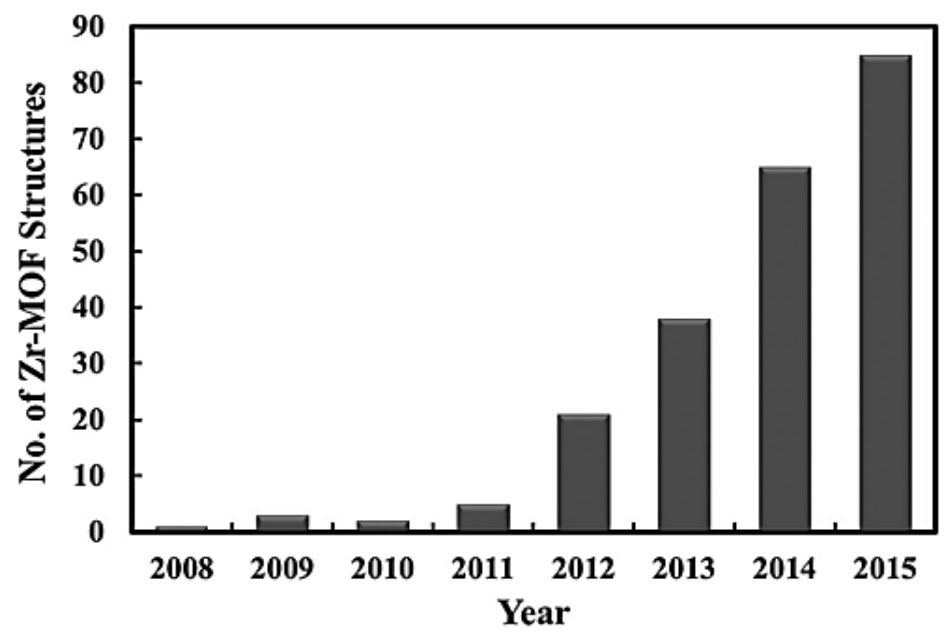

Figure 2.4-1: Yearly increase in reported new Zr-MOFs [4].

There are more than 100,000 MOFs produced using different synthetic methods each differing in regard to its building block composition, pore structure, pore sizes and functionality [1]. Each of these materials exhibit exclusive properties depending on their constituent parts. However, with respect to application, MOFs have been heavily criticized for their mechanical and thermal stability in harsh conditions, making production difficult on an industrial scale. Hence, in recent years, a major portion of the research in metal-organic frameworks was dedicated towards formulating recipes that produce MOFs with greater hydrothermal and chemical stability [1]. There are many selected MOFs whose development has led to assemblies manifesting great structural strength. One of the most prominent ones belong to the family of Zr-MOFs.

Zr-MOFs were initially produced as polycrystalline materials [36]. The strong ionic character of zirconium atoms quickly bonded with the oxygen atoms of BDC. This phenomenon slowed down the discrete growth of crystals, resulting in agglomerated polycrystalline powder [36]. The polycrystalline material was not only difficult to characterize through single XRD, but also limited in its range of industrial applications. Therefore, it was imperative that more efforts were to be dedicated to synthesizing discrete MOFs with large surface areas.

The synthesis of MOFs has grasped immense attention due to its potential structural modifications or its ability to be designed by altering synthesis routes. To broadly classify 
the different synthesis methods, MOFs can be produced by microwave assisted heating, sonochemical synthesis, electrochemical, mechanochemical, ionothermal, and conventional solvothermal synthesis [13], [43]. Each of these synthesis routes used in the production of MOFs greatly affect their properties and functionality [13]. Moreover, each of the methods have various merits and demerits associated with it and can be appropriately chosen based on the characteristics and availability of the material.

\subsubsection{Microwave Assisted Synthesis}

This synthesis technique is used to produce microporous MOFs by heating up a reaction mixture under solvothermal or hydrothermal conditions, where charged particles interact with an applied electromagnetic field [38], [42]. The source of charged particles can be polar solvent or a solid [43]. In this method, reactants are allowed to interact in an appropriate solvent and transferred to a Teflon vessel [22]. The vessel is then sealed and heated in a microwave, where the mixture is heated for a specific time [22]. On the application of the electric field, depending on the nature of reactants, the molecules either align themselves due to the oscillating electric field in a polar solvent, or a current is passed through the charges in a solid material [43]. As a result of the direct contact between the electric field and the charged molecules the kinetic energy of the molecules is increased which in turn increases the rate of crystallization [43], [44].

Advantages: Microwave assisted synthesis also provides phase selectivity, narrow particle size distribution, form nanoscale crystals, improved purity and facile morphology control [13], [43]. In addition, it also enables one to decrease the reaction time and quantity of required reactants [44]. In reference to the synthesis of Cr-MIL-100, using conventional hydrothermal synthesis required 4 hours at $200^{\circ} \mathrm{C}$ as opposed to requiring only $60 \mathrm{~min}$ at $210^{\circ} \mathrm{C}$ via microwave assisted synthesis, effectively decreasing the reaction time [13], [43].

Other remarkable MOFs produced via microwave assisted synthesis that successfully reduced the reaction times were Fe-MIL-100, HKUST-1, Fe-MIL-53, IRMOF-3 ( $\left.\mathrm{H}_{2} \mathrm{BDC}_{-} \mathrm{NH}_{2}\right)$, and ZIF-8 among others [13]. 
Disadvantages: This technique comes with drawbacks whereby the high temperatures may distort its structure due to thermal degradation, along with high cost of equipment and possibility of run-away reactions [45]. Thus, the constituents and energy inputs must be chosen appropriately for this application [43].

\subsubsection{Sonochemical Synthesis}

Sonochemical synthesis involved production of microporous materials by subjecting reaction mixture to high-energy ultrasonic wave [13], [43]. Unlike microwaves, the ultrasound waves do not interact with the reactant molecules. When a high energy ultrasonic wave is applied to the solution, high- and low-pressure regions are created. The areas of lowpressure experience pressure drop below the vapor pressure of the solvent, creating cavities. This causes the cavities to grow where the ultrasonic energy is accumulated by diffusion of the solvent into the cavities. When the bubble reaches its maximum size, the bubble collapses (also known as cavitation) releasing large amount of energy with rapid heating and cooling rates that induce shear forces in the mixtures [43]. Depending on the power output, the mixture experiences cavitation leading to rapid rise in temperature and pressure within the reactor [13]. Usually, the high-energy ultrasonic radiation generates a temperature and pressure of about $5000^{\circ} \mathrm{C}, 1000 \mathrm{~atm}$, respectively which is sufficiently large for otherwise prolonged reaction times [46]. On the other hand, cavitation produces microjets that activate the surface of locally available reactant solids [43], [46]. The simultaneous formation and breakdown of cavities generates shockwaves which lead to higher dissolution of reactants, along with bond cleavage and formation [43], [46], and eventually form crystallites.

Advantages: The production of nanoparticles is highly claimed to be an advantage of this application [13]; with one paper that confirms the statement with TEM micrographs [43]. Therefore, this claim is questionable. Nonetheless, this method achieves accelerated reaction rates, is eco-friendly, energy efficient, and permits room temperature synthesis [43]. Examples of polydisperse MOFs obtained from this synthesis method include, MOF-5 with crystal sizes of 5-25 $\mu \mathrm{m}$, HKUST-1 with 10-40 nm; while some uniform high-quality MOF 
crystals include PCN-6', PCN-6, IR-MOF-9, and -10 with crystal sizes between 1.5-2.0 $\mu \mathrm{m}, 4.5$ $6.0 \mu \mathrm{m}$, and 5-20 $\mu \mathrm{m}$, respectively [13].

Disadvantages: The main drawback of this method is lack of information about its specifications and performance [47]. A study of the hydrodynamics of sonochemical systems revealed that its is highly sensitive to the operational conditions. In the case of UiO-66-Ce, the rapid production of high temperatures and pressures also proved detrimental to the crystallinity and surface area [47].

\subsubsection{Electrochemical Synthesis}

Electrochemical synthesis is based on salt-free synthesis principle which solely counts on transportation of metal and linker in an electrolytic solution [48]. In this method, metal ions are continuously fed via anodic dissolution into the solution containing dissolved linker molecules in a suitable solvent [43]. When the voltage is applied across the electrodes, the high concentration of metal ions on the surface of the electrode react with the linker molecules in the electrolyte solution, coating the resultant MOF onto the electrode surface [49], [50]. Moreover, the repetitions of pulsed current can be optimized in this process [48]. During this reaction, metal ions are prevented from depositing on the cathode by using a protic solvent but also produces $\mathrm{H}_{2}$ gas. Thus, other reduced solvents such as acrylonitrile, acrylic, and maleic esters can be used [43]. Since commercialization of the production of MOFs involved large amount of anion formation, this process was aimed at eliminating the anions involved in the process [43]. Noteworthy $\mathrm{Cu}$ and Zn-based MOFs have already been synthesized by the BASF group with combinations of $\mathrm{Zn}, \mathrm{Cu}, \mathrm{Mg}$, and $\mathrm{Co}$ with organic molecules such as 1,3,5- $\mathrm{H}_{3}$ btc, 1,2,3- $\mathrm{H}_{3}$ btc, $\mathrm{H}_{2}$ bdc, and $\mathrm{H}_{2}$ bdc- $(\mathrm{OH})_{2}$ ) [43].

Advantages: The electrochemical synthesis of MOFs is a simple, environment friendly, and energy conserving process where crystals are synthesized at very short reaction times $\sim 30$ mins under mild conditions with easy scalability [50], [51]. 
Disadvantages: The side effects of using this synthesis method are corrosion of anode due to metal passivation, reduction in nucleation and crystal deposition rate due to metal oxidation, unreliable electric flow through the circuit, and production of toxic gas at cathode [50].

\subsubsection{Mechanochemical Synthesis}

Originated from the field of synthetic chemistry, mechanochemical synthesis is currently used in multicomponent reactions in the pharmaceutical industry, as well as solid-state chemistry, organic chemistry, and polymer science [43]. It utilizes a solvent-free synthesis procedure of MOFs by the exclusive application of mechanical force that induce mechanical cleavage of bonds with subsequent chemical reactions [43], [52], [53]. A typical synthesis uses grinding of metal oxide and ligands of specific compositions in the form of powder in a conventional ball mill or using a mortar and pestle [52]. The rotation and collision of ball bearings host mass transfer, similar to that of stirring, which induces kinetic energy that are in turn capable of controlling the heat generated, defects and dislocations within complexes, crystallite size or phase transformations [53]. This motion eventually leads to formation of MOF complexes. The product is observed by the change in the color, or by detection of specific odour of side products [52]. At times, metal ions are substituted with metal oxides, leaving only water as a by-product [52]. Moreover, in some cases, the addition of small quantity of solvent to the solid-solid mixture has proven to be virtuous as the solvent increases the agility of the mixture [52] referred to as LAG (Liquid-assisted grinding). Some MOFs produced using mechanochemical synthesis approach are [Cu(INA)2], HKUST-1, and others; while LAG products include [ $\left.\mathrm{Ln}(\mathrm{BTC})\left(\mathrm{H}_{2} 0\right)\right]$ ( $\mathrm{Ln}=$ lanthanoids such as $\mathrm{Y}, \mathrm{Sm}, \mathrm{Gd}, \mathrm{Tb}$, Dy, Er, Yb), Zn(Im)2, ZIF-4 and ZIF-8, among others [43]. This method asserts the environmentally friendly synthesis by eliminating the need for a solvent [53] and offers shorter reaction times with large quantifiable produce [52].

Advantages: The main features of this synthesis are a solvent-free synthesis and is environmentally friendly. Moreover, the crystals are readily obtained without a high energy requirement [43]. 
Disadvantages: This method may lead to unwanted amorphization, while characterization of the framework requires multiple analytical techniques [54].

\subsubsection{Ionothermal Synthesis}

Ionothermal synthesis, which was initially designed for zeolitic solids [55], [56] is one of the outstanding methods in MOF synthesis that utilize ionic liquids (ILs) instead of water or organic solvents [57]. This unique synthesis strategy is fairly recent and employs a straight forward procedure [13], involving the use of ionic liquids as reaction media, templates and/or charge compensating agents at an elevated temperature [58]. The reaction media is an attractive solvent due to virtually zero vapor pressure that provides increases the solubility of reactants [13]. This property of ILs allows the procedure to be conducted at both ambient and high temperatures without risking the development of autogenous pressure [56], [59]. Moreover, their ionic composition endures higher enthalpy of vaporization than that of water/volatile organic solvents [55], [57], [60]. Other advantages of this process include resistance to moisture, easy availability of inexpensive components, excellent solvating properties (dissolve both organic and inorganic precursors [61]), easy recyclability (catalytic property), high ionic conductivity and high thermal stability [13], [61]. Solvents typically used in this method are 1-alkyl-3-methylimidazolium (Figure 2.4.5-1) and deep eutectic solvents (DESs) [13]. The ionic liquid is an intrusive stimulus at room temperature that enhances slow reaction rates, nucleation and crystallization rates or accelerates the rate of reaction [13], [62] for Zr-MOFs. The UiO-66 was synthesized in [Omim] Cl, [ $\mathrm{Hmim}] \mathrm{Cl}$, and [Dmim] Cl [62]. It was observed that the crystallization was better for ILs with shorter alkyl chain [62].

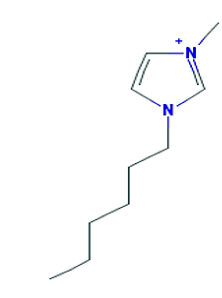

$[\mathrm{Hmim}]^{+}$

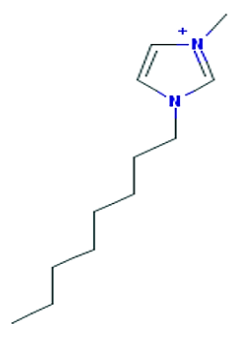

$[\mathrm{Omim}]^{+}$

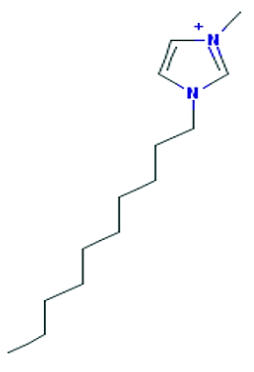

$[\text { Dmim }]^{+}$

Figure 2.4.5-1: Structural representation of 1-alkyl-3-methylimidazolium. 
Advantages: The ionic liquids used as a solvent in this method possess excellent solvating properties offering a large range of operational temperature $\left(-50^{\circ} \mathrm{C}\right.$ to $\left.200^{\circ} \mathrm{C}\right)[56]$.

Disadvantages: Due to the absence of solvents, this method ideally assumes no blockage of pores of the porous solids; however, this is only an ideal case as a small part of the cations in the ionic liquids decompose producing smaller template cations which serve as structure directing agents [63]. Thus, this affects the morphology and symmetry of the resultant framework.

\subsubsection{Conventional Solvothermal Synthesis}

Solvothermal synthesis is often resorted to due to its ease of synthesis and relatively mild reaction conditions [1], [9]. The conventional synthesis of MOF is through the application of electric heating, where temperature ranges are solvothermal and nonsolvothermal [43]. The solvothermal synthesis takes place in closed vessels under autogenous pressure above the boiling point of the solvent, while the nonsolvothermal synthesis takes place at or below the boiling point of the solvent under atmospheric pressure [43].

MOFs can be produced at room temperature or an elevated temperature, also known as conventional solvothermal synthesis. The synthesis following the room temperature, also known as direct precipitation method, involves synthesizing MOFs in a solvent at room temperature [43]. A few examples include MOF-199, IRMOF-0, MOF-177, MOF-5, HKUST-1, MOF-74, and others [43], [64]. Typically, this type of synthesis yields a poor crystal structure, mostly amorphous, as longer reaction times lead to degradation of the MOF [65]. Few exceptions to this case are MOF-199, MOF-5, MOF-74, and ZIF-8 [66] which possess good structural and thermal stability. However, most MOF syntheses face solubility issues at ambient temperature which makes its fabrication necessary at a higher temperature. Thus, along with ease of manipulation, conventional solvothermal synthesis at higher temperature remains most widely used in MOF synthesis.

A typical solvothermal synthesis procedure involves reacting metal salts with organic linkers in a formamide solvent at an elevated temperature for a pre-determined period [43], [50]. 
Once the synthesis is completed, the formulated solids are centrifuged and washed with fresh solvent. The centrifugation process is repeated to separate the suspended solids from the solvent which are further characterized using XRD and other characterization techniques to identify the material [45]. This type of synthesis has grasped immense attention due to its potential structural modifications by altering synthesis routes. The direct heating of mixture not only offers higher nucleation rate, efficiency, narrow particle size distribution, and phase selectivity, but also allows one to alter different reaction variables that help manipulate and control the morphology of the MOF [43], [67]. From a commercialization perspective, this process is simple, cost effective and the most straight forward method that allows easy manipulation of MOF characteristics. However, this method is exclusive to heat resistant materials, and its reaction time may be prolonged between days to weeks.

Advantages: In this method, the crystals and easily obtained and characterizable through single crystal XRD [54].

Disadvantages: Solvothermal synthesis produces a significant amount of solvent and mineral acids and is applicable to compounds resistant to heat [54].

\section{$2.5 \quad$ Objective}

Zr-based MOF UiO-66 is one of the most investigated MOFs because of its exceptional thermal and chemical stability. Its high porosity and resistance to adverse environment opens doors to applications in gas separation, catalytic reactions, drug delivery and florescence sensing among others [4]. However, this material falls below the expected industrial capacities and commercialization levels. Additionally, there is a lack of understanding of how the process variables (direction precipitation synthesis, stirring time, reaction time, reaction temperature) and composition (molar ratio, modulator concentration) variables affect the solvothermal synthesis of MOFs which leaves the crystallization/nucleation effects unclear and makes the tailorability of the material quite difficult. Therefore, in order to build MOFs with larger surface areas and pores sizes, this project aims at (a) conducting a systematic study on the effects of both process and compositional variables involved in the solvothermal formation of UiO-66 (b) modifying its 
morphology and/or properties by the individual addition of a new co-solvent, i.e. ethylene glycol (EG), diethylene glycol (DEG), and triethylene glycol (TEG). Moreover, this comprehensive study aspires to bridge the knowledge gap on how MOFs are formed by distinct mixtures using solvothermal synthesis conditions. This information can in turn serve as a protocol for researchers and engineers with designing MOF's functionalities to cater to the needs of the designated fields and large-scale production for industrial applications. 


\section{CHAPTER THREE \\ MATERIALS AND METHODS}

\subsection{Materials}

The chemicals used for various solvothermal synthesis of UiO-66 in this project are listed in Table 3.1-1. All materials were used without any further purification.

Table 3.1-1: Materials used for the synthesis of UiO-66.

\begin{tabular}{cc}
\hline Materials & Manufacturer/Supplier \\
\hline $\operatorname{DMF}(99.8+\%)$ & Alfa Aesar \\
$\operatorname{ZrCl}_{4}(\geq 99.5 \%)$ & Aldrich \\
$\mathrm{H}_{2} \mathrm{BDC}(98 \%)$ & Aldrich \\
Acetic acid (99.7\%) & BDH Aristar \\
Ethylene Glycol (99\%) & Alfa Aesar \\
Diethylene glycol (99\%) & Alfa Aesar \\
Triethylene glycol (99\%) & Acros Organics \\
\hline
\end{tabular}

\subsection{Synthesis Routes}

\subsubsection{Synthesis of UiO-66 without co-solvent}

The following synthesis routes were adopted in order to study the various aspects of this project. For all the experiments, the molar composition of $\mathrm{ZrCl}_{4}$ was kept constant at 0.343 mmol and used as a basis for all other component concentrations. The chemical composition was adopted from the originally published paper by Cavka et al. [37] with the addition of acetic acid. A typical Ui0-66 sample was prepared using Scheme 1 with equimolar amounts of 1:1 $\mathrm{ZrCl}_{4}$ and $\mathrm{H}_{2} \mathrm{BDC}$ dissolved into of DMF in a $150 \mathrm{ml}$ glass reactor vessel (Kemtech America, P170004) by using a magnetic stirrer. The acetic acid was then added to the mixture and the solution was left to stir at 1 unit. The vessel was then closed off with seal plugs wrapped with a layer of Teflon tape. The solution was left to stir at 1 unit for $24 \mathrm{~h}$ as the experimental set up represented in Figure 3.2.1-1. The precursor solution was then subjected to a heating at $120^{\circ} \mathrm{C}$ for 24 hours. The formulated crystals in the glass reactor 
vessel is shown in Figure 3.2.1-2. Upon completion, the solids were decanted and recovered by centrifugation at $12000 \mathrm{rpm}$. The solids were then re-dispersed via sonication into $5 \mathrm{ml}$ of fresh DMF solution and allowed to stand overnight at room temperature. The centrifugation and sonication processes were repeated with fresh DMF for a second overnight standing, after which the solids were left to dry at room temperature in a petri dish. The resultant solids were in the form of dry powder material as shown in Figure 3.2.1-2.

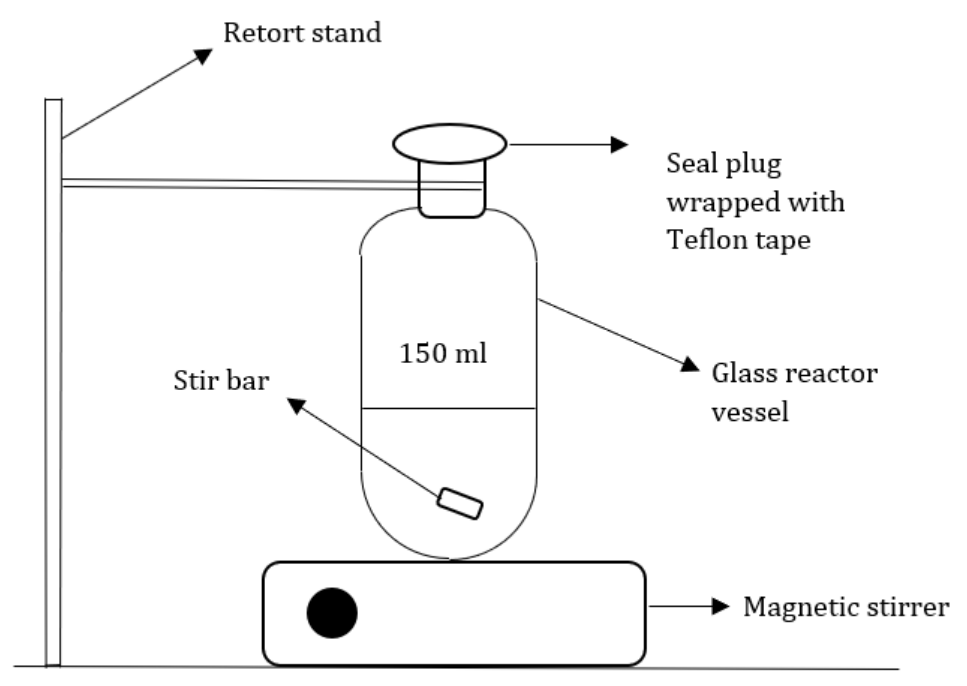

Figure 3.2.2-1: Schematic diagram of the experimental set up for solvothermal synthesis of UiO-66 crystals. 

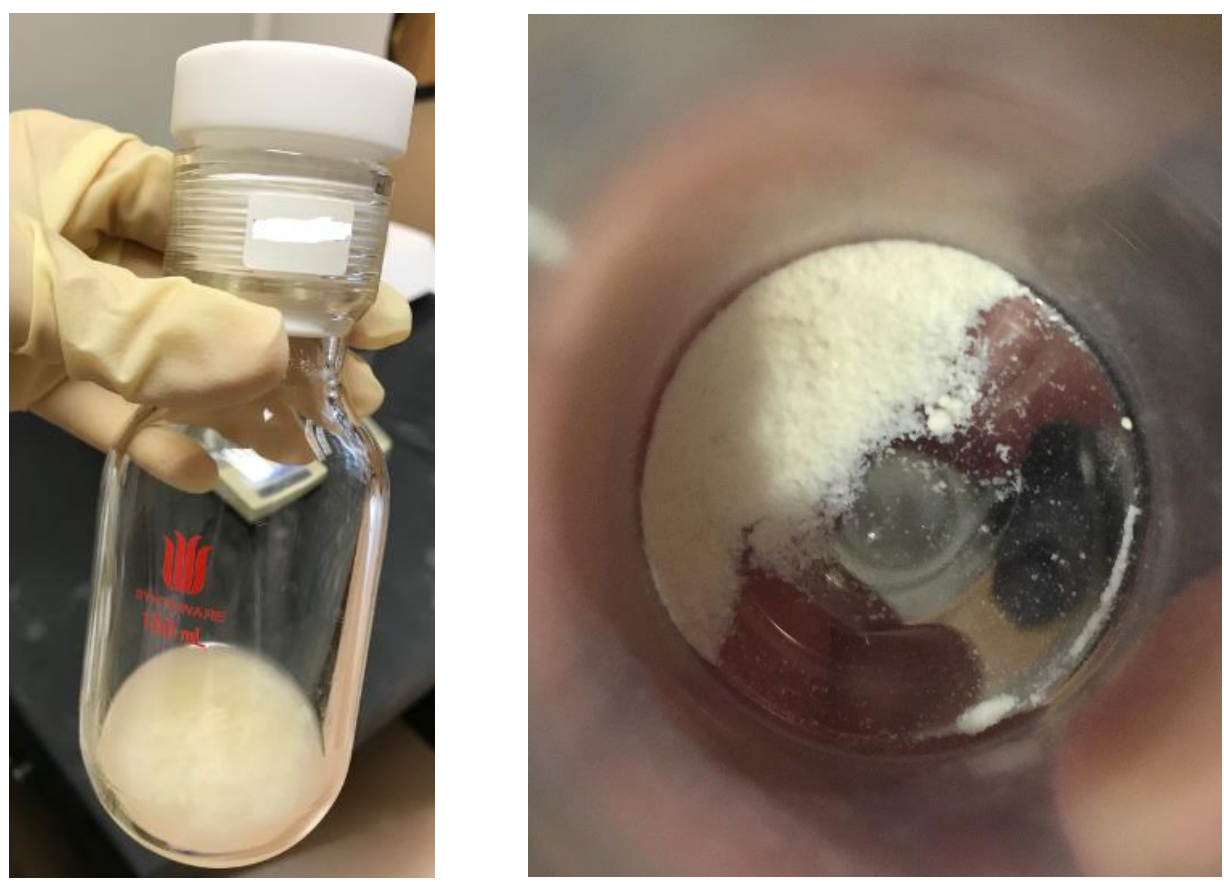

Figure 3.2.1-2: Formulated UiO-66 crystals before drying (left) and after drying(right).

The experiments performed based on this scheme were the effect of stirring time, reaction time, temperature, and modulator (acetic acid) concentration.

Scheme 1: Synthesis composition of UiO-66 samples

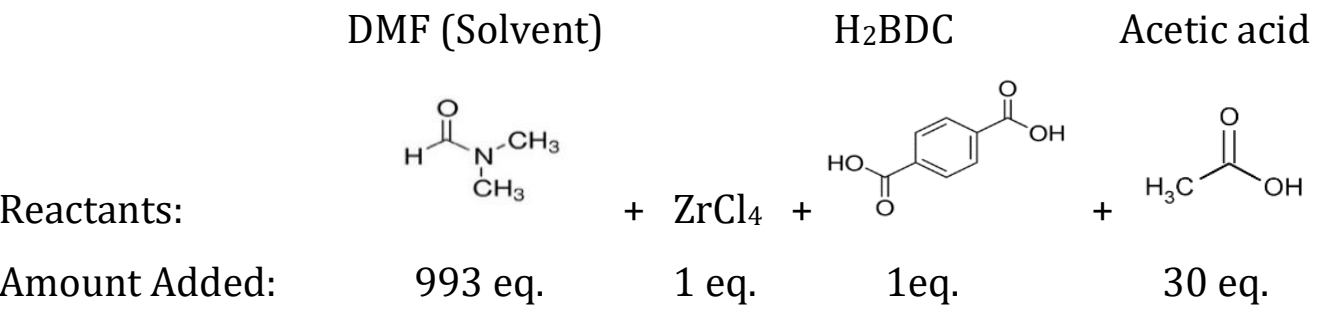

\subsubsection{Synthesis of UiO-66 with co-solvent}

Scheme 2, a modification of Scheme 1 with a higher DMF content, was also used for the effect of co-solvent concentration on the morphology of UiO-66. Due to the unsuccessful results with this synthesis route, Scheme 3 was adopted and used to analyze the effect of molar concentration, effect of co-solvents, and the concentration of co-solvents on the morphology of UiO-66. 
The compositions of scheme 1 were adjusted to the published paper by Lu et al. [68] with the addition of a co-solvent. UiO-66 was synthesized using Scheme 3 by first pouring a 30eq. amount of ethylene glycol or diethylene glycol or triethylene glycol as a co-solvent with respect to $\mathrm{ZrCl}_{4}$ into $\mathrm{DMF}$ in a $150 \mathrm{ml}$ glass reactor vessel. The solvent mixture was homogenized by placing the glass reactor vessel with a magnetic stirrer for 5 mins. The solid reactants, i.e. $\mathrm{ZrCl}_{4}$ and $\mathrm{H}_{2} \mathrm{BDC}$ were then added to the solvent mixture. Once the solute particles dissolved, acetic acid was further added to the solution and left to stir for $24 \mathrm{~h}$. The vessel was then heated $120^{\circ} \mathrm{C}$ for 4 days under static conditions. Once the reaction was complete, the washing procedure was repeated as mentioned in Section 3.2.1 to obtain the Ui0-66 crystals. A schematic of the general overall process is depicted in Figure 3.2.2-3.

Scheme 2: Synthesis composition of UiO-66 samples

DMF (Solvent) $\quad \mathrm{H}_{2} \mathrm{BDC} \quad$ Acetic acid

Reactants:

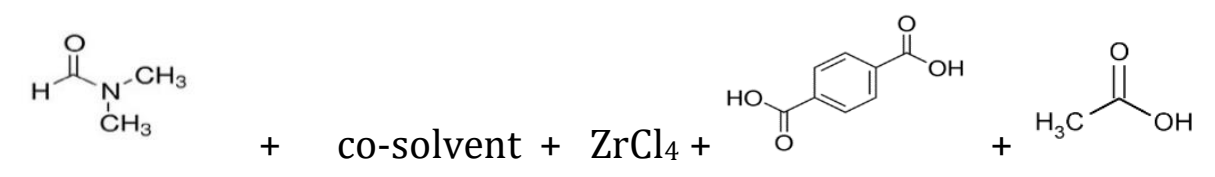

$\begin{array}{llll}\text { Amount Added: } \quad 1500 \text { eq. } & \text { Variable } \quad 1 \text { eq. } \quad 1 \text { eq. } & 30 \text { eq. }\end{array}$

Scheme 3: Synthesis composition of UiO-66 samples

DMF (Solvent) $\quad \mathrm{H}_{2} \mathrm{BDC} \quad$ Acetic acid

Reactants:

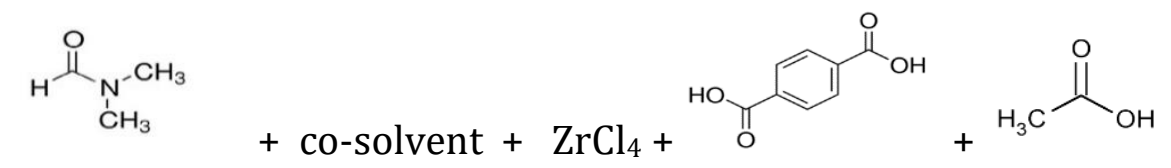

Amount Added: 866 eq. $\quad$ Variable $\quad 1$ eq. $\quad 1$ eq. $\quad 160$ eq. 


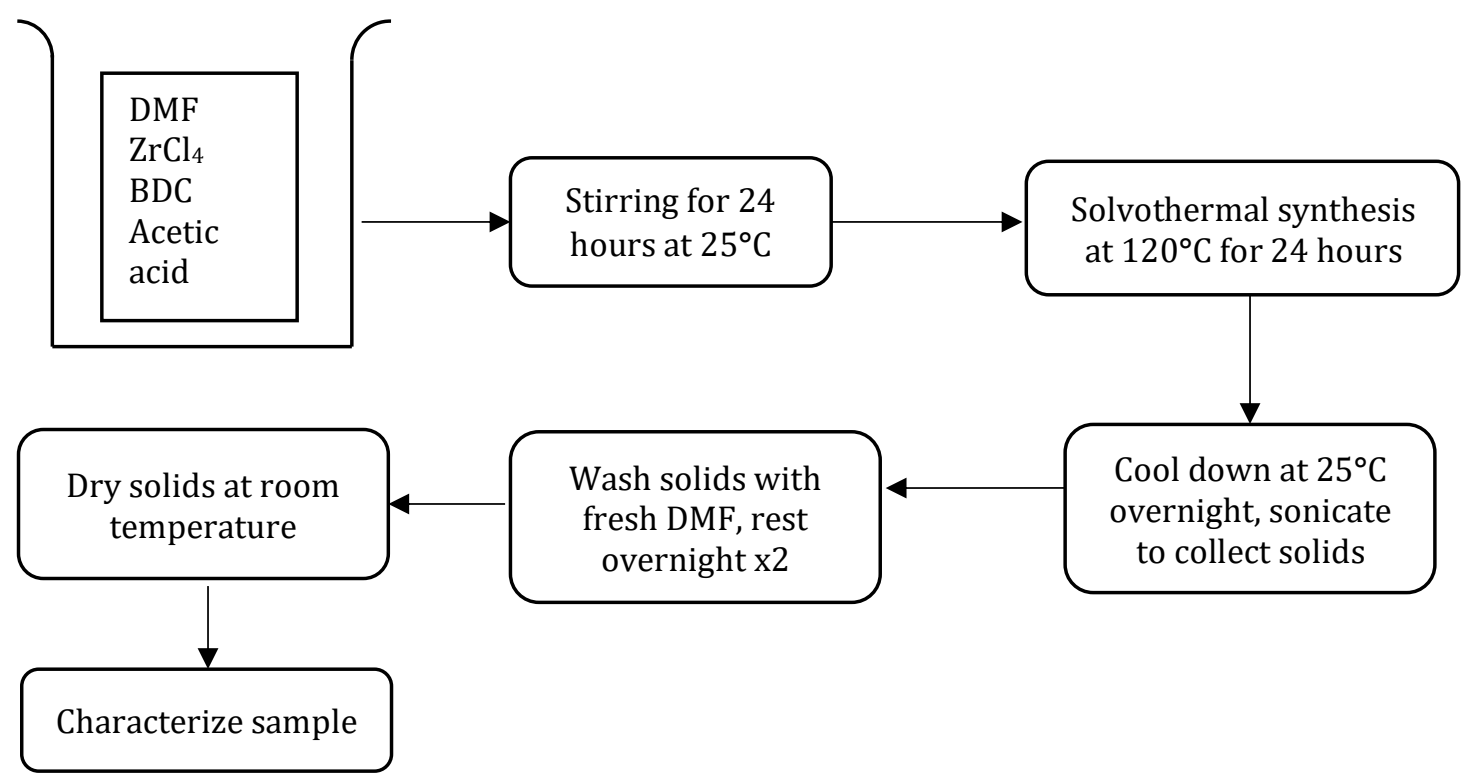

Figure 3.2.2-3: Schematic diagram of typical solvothermal synthesis of UiO-66 crystals.

\subsection{Experimental Methods}

The characterization of Ui0-66 samples involved multiple analytical instruments to form a conclusive judgement on their morphology, properties and growth mechanism. These instruments were the powder X-ray diffraction (PXRD), scanning electron microscope (SEM) imagining, Brunauer-Emmett-Teller (BET) surface area analysis and thermogravimetric analysis (TGA). Their identity, crystallinity and phase purity were determined by PXRD. The SEM imaging was utilized to reveal the morphology of the particles, while the BET revealed the specific surface area. Furthermore, TGA experiment was also performed detect the loss of sample over time with change in reaction temperature. The aim of this chapter is to describe the basic theories of each analytical technique in contemplation of validating the observations and highlighting new findings. 


\subsubsection{Powder X-ray Diffraction (PXRD)}

PXRD is extensively used for identifying different phases and purity of microporous crystalline materials such as zeolites and metal-organic frameworks. X-rays are generated in an X-ray tube containing a source of electrons and two metal electrodes. When a high voltage is applied across the electrodes, the electrons are rapidly drawn to the metal/target electrode which is usually copper or nickel. The excited electrons return to the ground state by emitting the electromagnetic radiation X-rays. Depending on the sample, the X-rays are diffracted from the crystallites containing ordered and evenly atoms on a certain plane. The intensities of the X-ray, a function of $2 \theta$, recorded by the detector are the diffracted rays that satisfy the Bragg's diffraction condition.

$$
\lambda=2 d_{h k l} \sin \theta[69]
$$

The Bragg's law is given by equation (3.3.1-1), where $\lambda$ is the constant wavelength of the $\mathrm{X}$ ray, $d_{h k l}$ is the distance between planes with Miller indices $h k l$ and $\theta$ is the angle of the incident X-rays.

For all samples, the powder X-ray diffractograms were obtained with a Phillips PANanalytical diffractometer model number PW3710, manufactured in Amsterdam, using a $\mathrm{CuK} \beta$ with Ni filter and a wavelength, $\lambda=1.54 \AA$. The powder X-ray diffraction patterns were measured for a $2 \theta$ range of $5-35^{\circ}$ with a step size of $0.02^{\circ}$ and 2 seconds per step. Figure 3.3.1-1 and Figure 3.3.1-2 show a pictorial representation of the Bragg reflection of incident X-rays by the atomic particles and a typical powder diffractogram of Ui0-66 prepared by solvothermal synthesis process of $\mathrm{ZrCl}_{4}: \mathrm{H}_{2} \mathrm{BDC}$ (1:1 ratio) with DMF (866 eq.) and acetic acid (160 eq.) heated at $120^{\circ} \mathrm{C}$ for 24 hours. 


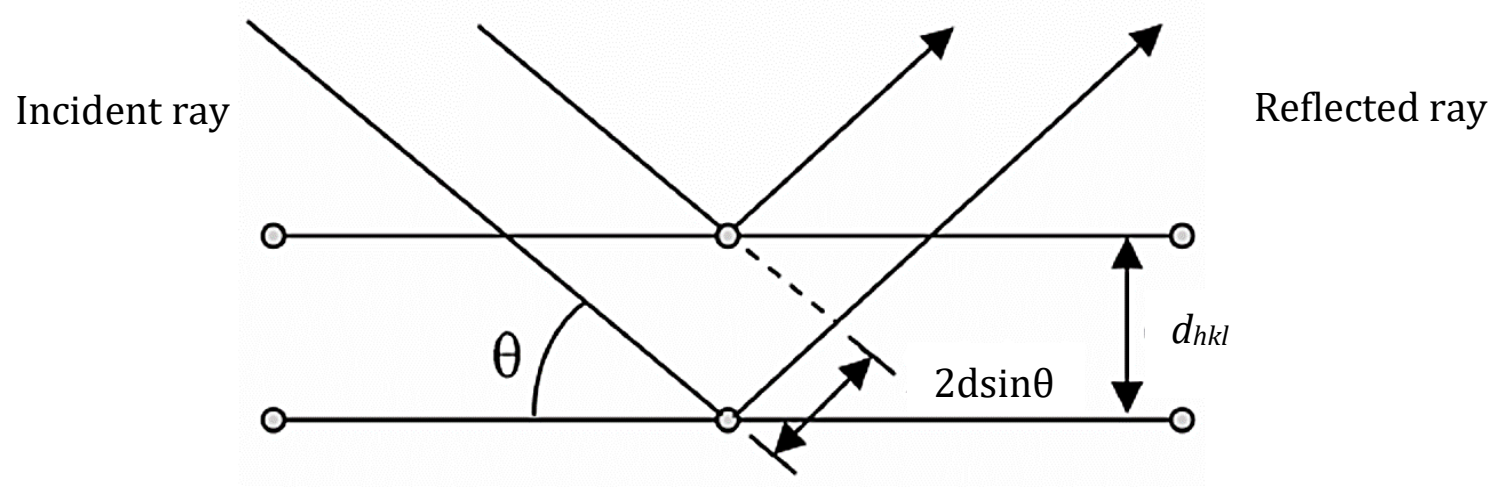

Figure 3.3.1-1: Schematic representation of Bragg's law of diffraction.

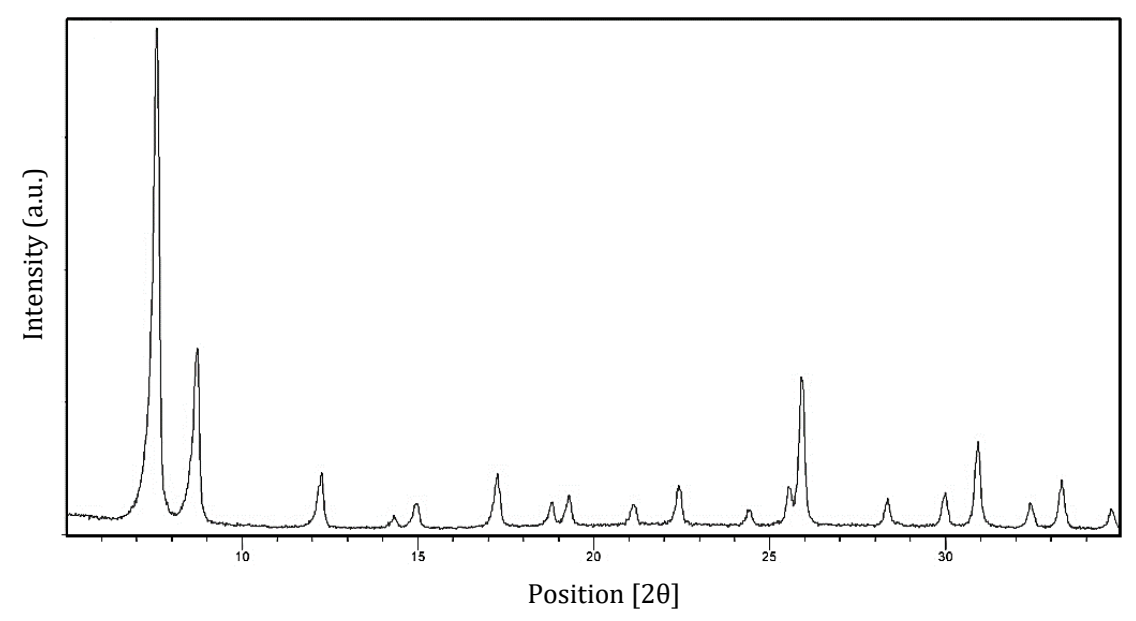

Figure 3.3.1-2: PXRD pattern of UiO-66.

The reflections at different $2 \theta$ angles are usually compared to the available diffraction pattern in literature to determine the structure of an unknown material. The diffraction pattern represents the reflections of the X-rays from atomic particles on specific planes represented by three integer indices $h k l$, which used to describe the orientation of the x-ray reflecting planes. The interplanar distance $d$ is associated with the $h k l$ indices given by the equation (3.3.1-2) for a primitive cubic cell symmetry where $a$ is length of edge of unit cell.

$$
d=\frac{a}{\sqrt{h^{2}+k^{2}+l^{2}}} \quad \text { [69] }
$$




\subsubsection{Scanning Electron Microscope (SEM)}

SEM is a characterization tool that reveals surface of the particles. It is essentially a high magnification electron microscope that scans the surface of the samples by focusing an electron beam and is extensively used to determine the particle shapes and sizes of microporous materials. SEM was used for all samples to determine the surface characteristics of crystals, size distribution and shape.

The SEM analyses were performed by dispersing the particles onto a metal stub surface covered with carbon tape. The metal stubs were then loaded onto the sample holder and gold coated inside a high-pressure chamber. The prepared samples were then mounted onto the SEM of a JEOL machine, model number 6610LV. A sample SEM image of the Ui0-66 assynthesized sample is shown in Figure 3.3.2-1. The sample was synthesized via solvothermal synthesis process of $\mathrm{ZrCl}_{4}: \mathrm{H}_{2} \mathrm{BDC}$ (1:1 ratio) with DMF (866 eq.) and acetic acid (160 eq.) heated at $120^{\circ} \mathrm{C}$ for 24 hours. The crystals sizes were determined manually using the scale bar and calculated for average size and standard deviation.

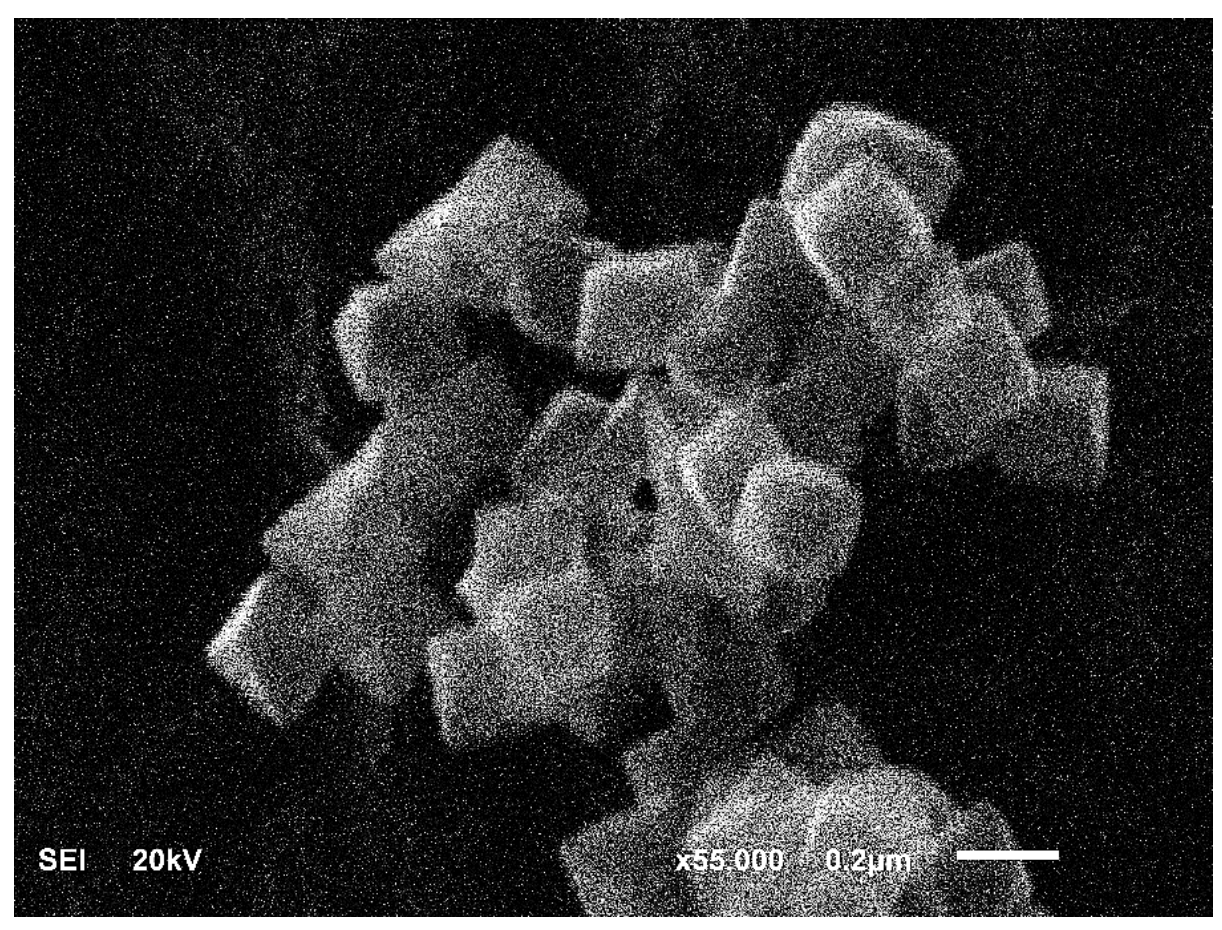

Figure 3.3.2-1: SEM image of as-synthesized UiO-66 sample. 


\subsubsection{Attenuated Total Reflection Infrared (ATR-IR)}

ATR-IR spectroscopy is an analytical tool used in combination with infrared spectroscopy which is used to identify organic, polymeric and some inorganic materials. This technique requires no sample preparation and can be used for both solids and liquids. The sample is usually clamped to the ATR crystal in a way that the beam is passing through the material directly instead of an intermediate medium. ATR works on total internal reflection that generates an evanescent wave. In this technique, an infrared light beam is passed through the ATR crystal such that it reflects at least once off the surface in contact with the material which extends into it. The depth of penetration of the wave ranges from $0.5 \mu \mathrm{m}$ and $2 \mu \mathrm{m}$ depending on the wavelength of light, the angle of incidence and refraction for the ATR crystal and the material. The reflected wave from the ATR crystal is then collected by the detector. The reflections are analyzed by computer program using Fourier transforms to obtain a single-beam infrared spectrum. The FTIR (Fourier transform infrared spectroscopy) spectrum is generally plotted as an intensity versus wavenumber $\left(\mathrm{cm}^{-1}\right)$ which is the reciprocal of the wavelength. The samples for which the XRD and SEM analyses were insufficient were further characterized using ATR-IR instrument (manufacturer: Agilent, and model: Cary 630) over a wavenumber range of $600-4000 \mathrm{~cm}^{-1}$. A typical IR spectrum of the as-synthesized UiO-66 via solvothermal synthesis process of $\mathrm{ZrCl}_{4}: \mathrm{H}_{2} \mathrm{BDC}$ (1:1 ratio) with DMF (866 eq.) and acetic acid ( 160 eq.) heated at $120^{\circ} \mathrm{C}$ for 24 hours is depicted in Figure 3.3.3-1. 


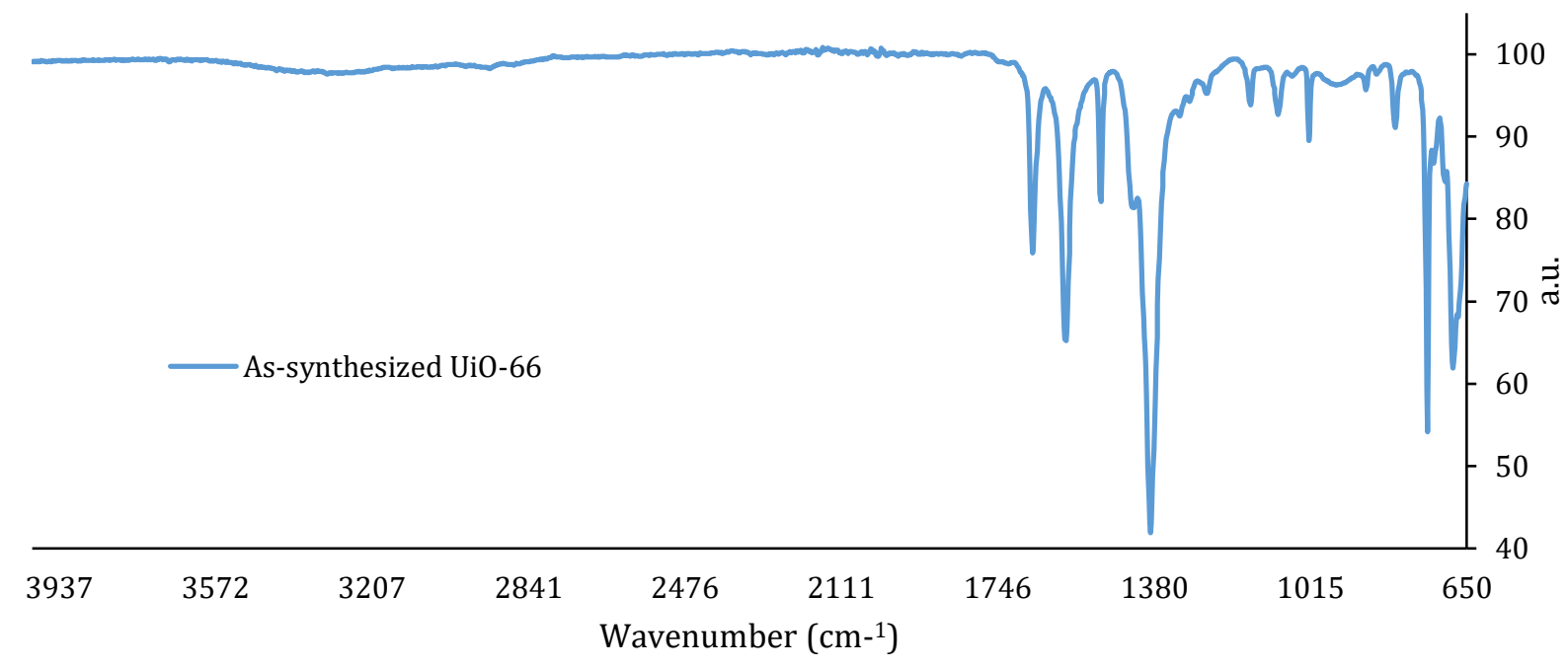

Figure 3.3.3-1: FTIR Spectrum of as-synthesized UiO-66. (See Table 4.7.2-3 for vibrational modes).

\subsubsection{Thermogravimetric Analysis (TGA) and Differential Scanning Calorimetry (DSC)}

TGA is a thermal analysis that indicates the rate of decomposition of a material overtime as a function of the temperature. The data reveals the phase transitions, heat absorption and desorption of the sample along with other oxidation/reduction states. The TGA in this study was carried out in a TGA Q500 instrument. Approximately $10 \mathrm{mg}$ of the UiO-66 sample was placed in platinum sample pan. The TGA analysis was performed with nitrogen gas at a total flow of $100 \mathrm{~mL} / \mathrm{min}$ (Nitrogen for balance and sample gas are at 40 and $60 \mathrm{~mL} / \mathrm{min}$, respectively). The TGA profile was collected by heating the sample from room temperature to $1000^{\circ} \mathrm{C}$ at a ramping rate of $10^{\circ} \mathrm{C} / \mathrm{min}$. In addition, DSC is a part of the TGA that indicates the phase transformations that take place within a sample. The DSC equates based on the heat requirement for transitioning from one phase to another from previous reference state. The TGA and DSC of as-synthesized UiO-66 is presented in Figure 3.3.4-1. The sample was produced using the solvothermal synthesis process of $\mathrm{ZrCl}_{4}: \mathrm{H}_{2} \mathrm{BDC}$ (1:1 ratio) with DMF (866 eq.) and acetic acid (160 eq.) solution heated at $120^{\circ} \mathrm{C}$ for 24 hours. 


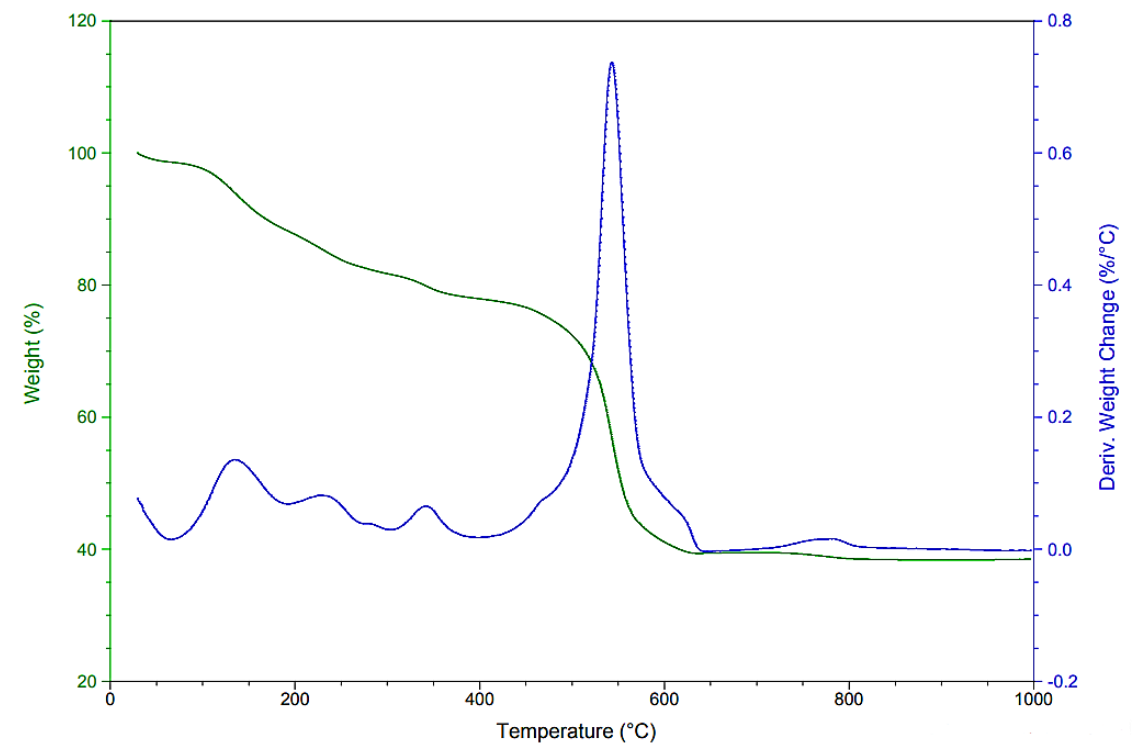

Figure 3.3.4-1: TGA(green) and DSC(blue) profiles of as-synthesized UiO-66.

\subsubsection{Brunauer Emmett and Teller (BET) Surface Area Measurements}

BET method is used to characterize the surface area and pore size of porous materials. In this method, the surface of $0.007 \mathrm{~g}$ of the porous material was purged by a gas at liquid nitrogen temperature $\left(-196.2^{\circ} \mathrm{C}\right)$. The surface area was then determined by calculating the amount of gas adsorbed as a monomolecular layer on its surface. The gas is adsorbed by the weak van der Waal forces between the porous material and the gas molecules.

The BET surface area of the material is calculated by the equation (3.3.5-1) where $v$ is the adsorbed volume of gas, $v_{m}$ is the adsorbed monolayer volume, $p$ is the equilibrium gas pressure, $p_{0}$ is the saturation pressure, and $c$ is the BET constant. This is a linear equation with $y$-axis as the left-hand side of the equation and $x$-axis is $p / p_{0}$.

$$
\frac{1}{v\left[\left(\frac{p}{p_{o}}\right)-1\right]}=\frac{c-1}{v_{m} c}\left(\frac{p}{p_{o}}\right)+\frac{1}{v_{m} c} \quad \text { [70] }
$$

The $y$-intercept and slope of this function are used to obtain the constants $c$ and $v_{m}$. The specific surface area of the sample $\left(S\right.$, surface area per unit mass $\left.\left[\mathrm{m}^{2} / \mathrm{g}\right]\right)$ can then be 
calculated by using equation (3.3.5-2), in which $N$ is Avogadro's number (number of molecules per mole), $A$ is the area of cross-sectional of a single adsorbed gas molecule, $m$ is the mass of materials used in the experiment and 22,400 represents the Standard Temperature and pressure (STP) volume of one mole of gas. This surface area is given in units of area/mass (e.g., $\mathrm{m}^{2} / \mathrm{g}$ ), which can be converted to a volume-specific surface area by multiplying by the material density.

$$
S=\frac{v_{m} N A}{22,400 \times m}
$$




\section{CHAPTER FOUR}

\section{FACTORS INFLUENCING THE MORPHOLOGY OF MOFS}

Conventionally, MOFs are largely synthesized via solvothermal synthesis where process and compositional parameters can be strategically adjusted to generate diverse morphologies with different thermal and chemical stabilities. Compositional parameters include concentration of the starting materials, $\mathrm{pH}$ of the reaction, and solvent; while process parameters are reaction time, temperature, and pressure [43]. Both these parameters have been extensively examined to find their effects on the crystal structure. The latter parameter, specifically temperature and reaction time have been varied in the solvothermal production of UiO-66 to study the effect on its structure and stability [64], [71]. The influence of reaction temperature, molar ratios of starting materials, and $\mathrm{pH}$ of the reaction mixture impacts the morphology of the final material [72]-[74]. Since minute changes in reaction variables drastically affect its morphology, a precise control over their morphology at the molecular level becomes a challenging process. To address this problem, a fundamental and systematic investigation of parameters on the fabrication of UiO-66 via solvothermal synthesis was conducted to help bridge the knowledge gap and may offer better control over its morphology. This exercise generated a synthesis protocol to serve as a pivotal platform for designing MOFs functionality. As UiO-66 remains an excellent candidate in diverse industrial applications because of its aforementioned properties such as tunable composition, structural diversity, inherent large surface areas, uniform tunable cavities and tailorable chemistry [32], [41], the goal of this project was achieved by studying the effect of individual process and compositional parameters through systematic manipulation on the morphology of UiO-66 in conventional solvothermal synthesis process.

\subsection{DIRECT PRECIPITATION SYNTHESIS}

\subsubsection{Introduction}

Syntheses of MOFs at room temperature were successfully achieved about a decade ago on ZIF-8, MOF-5 and HKUST-1 [64], [75], [76]. These MOFs are perfectly crystalline, efficient and require $<24 \mathrm{~h}$ synthesis time which reduces the cost of production and energy 
requirement [64], [75], [76]. Recently, Hupp and Farha's group reported successful synthesis of various MOFs at room temperature, including UiO-66 and its derivatives [77], [78]. The method described in their experiment required introduction of SBUs in the reaction mixture with a synthesis time for $\mathrm{UiO}-66$ of $18 \mathrm{~h}$ as opposed to introducing pure precursors (i.e., $\mathrm{ZrCl}_{4}$ and BDC) as in the original synthesis recipe. The results for UiO-66 synthesized at $25^{\circ} \mathrm{C}$ [79], emphasized that an SBU of zirconium clusters must form first at an elevated temperature, followed by the addition of the organic ligand at room temperature. This procedure did not eliminate the high temperature demand, but rather reduce it. In addition, the sources also reported using a more expensive zirconium source for generating SBUs. Thus, the main motivation behind synthesizing solution-based UiO-66 using the published recipe at room temperature was to minimize the energy demand on the conventional solvothermal syntheses that requires the use of elevated temperatures.

\subsubsection{Results and Discussion}

The main intention of this experiment was to cut down the energy demand of the elevated temperature synthesis of UiO-66 by carrying out direct precipitation synthesis. The UiO-66 synthesis was conducted at room temperature using Scheme $\mathbf{1}$ was stirred for 30 days until

a precipitate was formed. In order to compare the crystallinity of the product obtained using the direct precipitation method, an as-synthesized sample was also prepared using Scheme 1 , where the mixture was stirred for 24 hours and heated to $120^{\circ} \mathrm{C}$ for 24 hours. The resulting as-synthesized sample was a white power material which was characterized using PXRD and SEM.

The SEM images of the samples are shown in Figure 4.1.2-1. The solids prepared using the direct precipitation method showed the presence of a large surface, almost featureless. The surface seemed to contain very small ill-defined particles. There was no identification of distinct shape or characteristics in this sample. The as-synthesized solids exhibited faceted discrete particles with an average diameter of $152 \pm 12 \mathrm{~nm}$. 


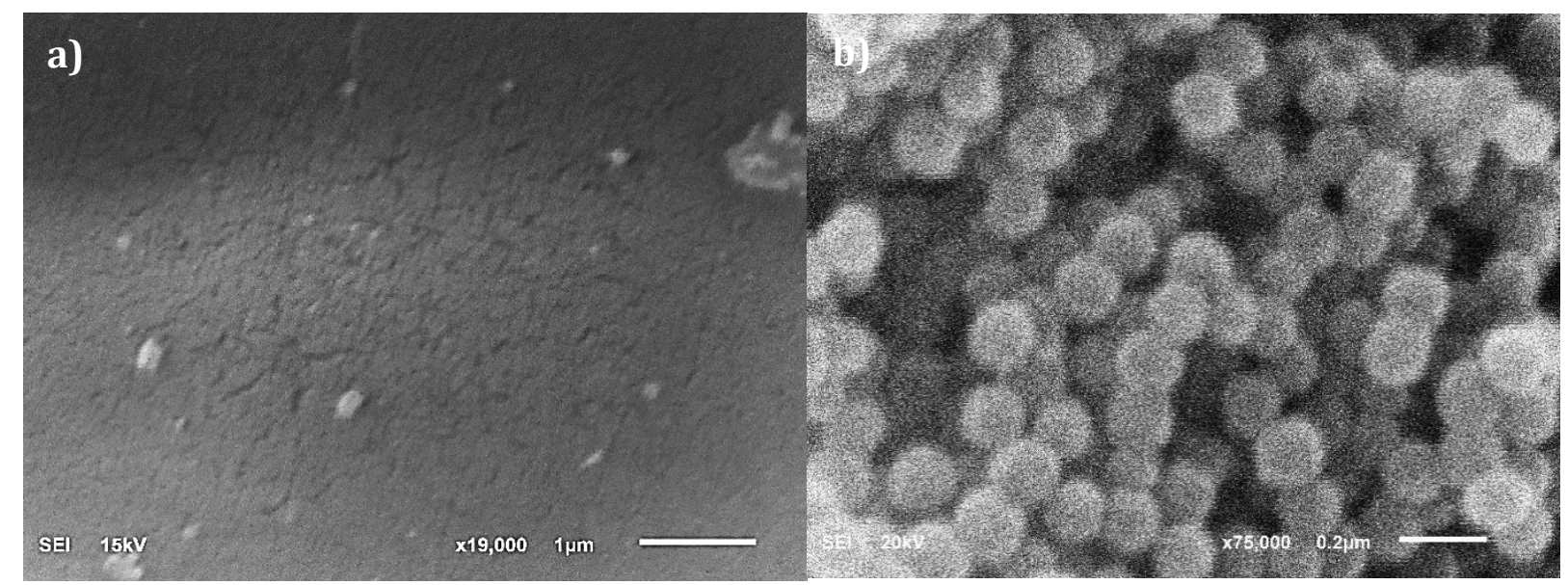

Figure 4.1.2-1: SEM images of UiO-66: a) synthesized using the direct precipitation method, and b) as-synthesized sample.

The PXRD patterns displayed in Figure 4.1.2-3 show the crystallinity and phase purity of the samples and is in good agreement with the literature reported diffractogram of UiO-66 and desolvated UiO-66 crystals (Figure 4.1.2-2) [41]. Both the room temperature sample and the as-synthesized sample were in fine agreement with the as-synthesized UiO-66 PXRD pattern reported in the literature [37]. The as-synthesized sample exhibited no peak splitting or peak broadening with maximum observed Bragg peak intensities over the entire $2 \theta$ angle indicating the formation of highly crystalline UiO-66. The PXRD pattern of the room temperature synthesized sample showed peak broadening and reduced peak intensities for the entire $2 \theta$ range demonstrating reduced crystallinity. These two features were possibly due to the presence of ill-defined fine particles in the sample, which was supported by the SEM image. Moreover, there was no peak shifting in the PXRD patterns, implying that the topology of both samples was comparable. 


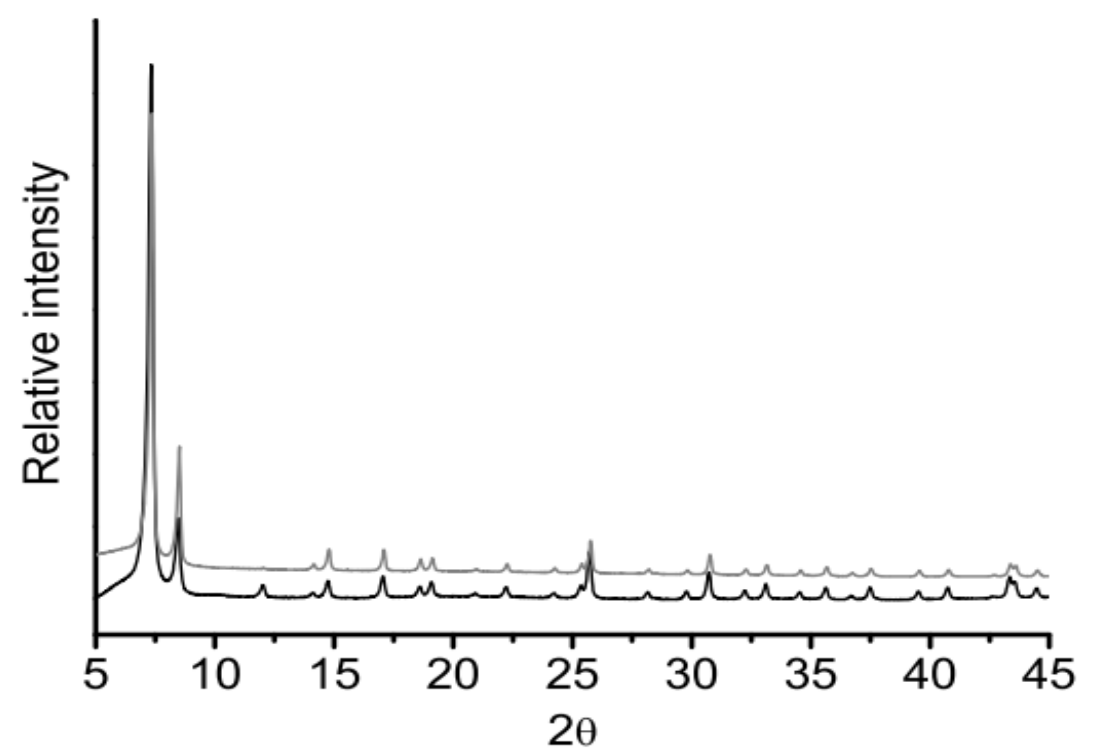

Figure 4.1.2-2: PXRD pattern of UiO-66 (black) and desolvated (grey) UiO-66 [37]

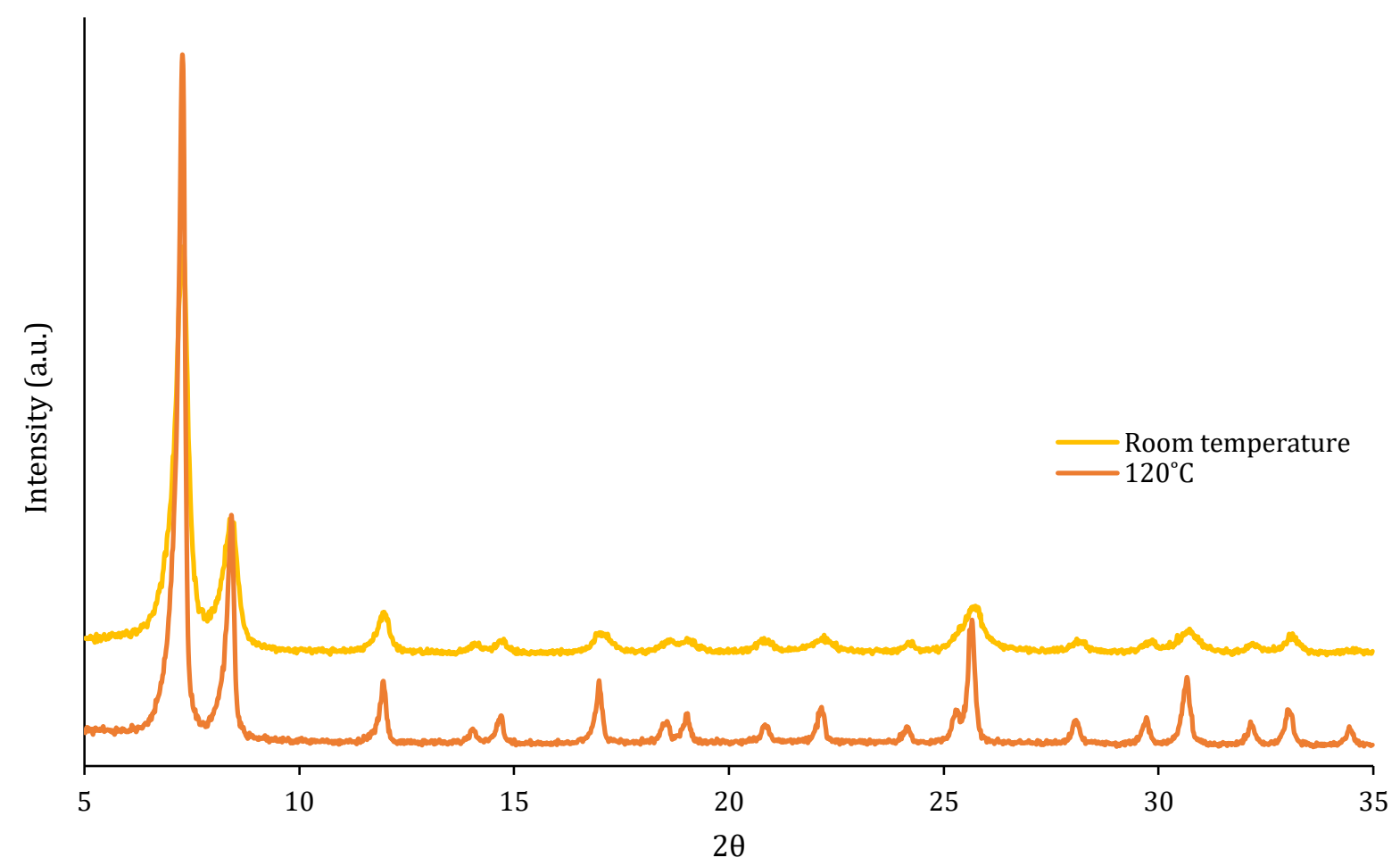

Figure 4.1.2-3: Comparison of XRD patterns of UiO-66 synthesized at room temperature and at $120^{\circ} \mathrm{C}$. 


\subsubsection{Conclusion}

This work demonstrated the production of solution-based synthesis of UiO-66 solely at room temperature. The results indicated that, using published UiO-66 composition and chemicals, the formation of UiO-66 at room temperature is achievable.

Some important observations drawn from Hupp and Farha's group [77] were that, the room temperature synthesized UiO-66 was crystalline but not equivalent to the elevated temperature sample. The PXRD pattern of the room temperature sample showed broadened peaks indicating small and poor crystallinity particles. The SEM images showed a particle size of about $250 \mathrm{~nm}$, while BET surface area of the sample was $1450 \mathrm{~m}^{2} / \mathrm{g}$, which was close to the reported UiO-66 with defects [77], [80]. These observations are consistent with the experimental results in this study. The peak broadening of the PXRD pattern or directly precipitated sample was attributed to the very small particle size which was barely visible under the given SEM resolution. The cryptic appearance of the room temperature sample could be a result of the production of very small crystal size. This result was expected because low synthesis temperature decreases the solubility of the reactants which may lead to incomplete hydrolysis of the $\mathrm{Zr}$ salt by BDC linker. Consequently, the slowing down of this crucial step causes irregular formation of SBUs which in turn leads to formation of ill-defined UiO-66 crystals. Therefore, it is not possible to achieve high crystallinity under room temperature conditions since the crystallization rate is impractically slow.

Hence, it can be concluded that pure phase UiO-66 crystals can be successfully synthesized at room temperature with elongated reaction time; however, the crystallinity is marginal. As the study suggests that even employing the synthesis with an SBU failed to deliver the crystallinity as achieved in elevated temperature synthesis [77]. Thus, room temperature synthesis can be adopted only to comprise the quality of the material in terms of both porosity and crystallinity. Given the sluggish crystallization rate of UiO-66 at room temperature along with marginal crystallinity, the following studies were all conducted at elevated temperatures. 


\subsection{EFFECT OF STIRRING TIME}

\subsubsection{Introduction}

If not all, the effect of most reaction variables on the morphology of MOFs have been previously touched upon in the literature; however, stirring time of the MOFs precursor solutions is one vital factor that has often been overlooked. Most MOF syntheses indiscriminately adopt a synthesis period of 24 hours. However, the influence of stirring time on the framework of MOFs, to the best of our knowledge, has not been studied yet.

Mazzarotta et al. [81] demonstrated that the effect of stirring was readily reflected on size distribution, number of generated fragments, and the morphology of the crystals. It was found that initial stirring of the solution showed high fragmentation of crystals due to the surface defects of the crystals, even with low energy collisions. This phenomenon is termed as abrasion, where surface particles are shattered through enforced collisions with one another and parts of the equipment. The reduction in attrition occurred once the largest crystals are dissolved, while the remnant crystals continued to collide into one another fatiguing the particles. Consequently, these particles required additional stirring. In addition, physical agitation also enhanced the solubility of the solute by increasing the probability of collision of molecules [81]. The constant collisions increased the kinetic energy of the molecule, which as a result, raised the temperature of the system [43] leading to product formation. From a hydrodynamic perspective, the continuous stirring of the solution causes increased friction between solute particles [81] that reduces the boundary layer thickness of the solute particles [82]. Eventually, the crystals dissolve into the solution.

In general, stirring is said to ensure homogeneity of the solute concentration [83], while also establishing a uniform temperature and constitution [82]. It stimulates surface diffusion and molecular integration in a supersaturated solution. It also increases the interaction of the reactant molecules and transfers it into the bulk of the solution [82]. Hence, the effect of stirring on a reaction within a solution and, from a broader perspective, on the morphology of MOFs cannot be neglected. Thus, we hypothesized that the duration of stirring time could indirectly affect the structural properties of UiO-66, and MOFs in general as well. To prove our hypothesis, this section analyzed the structural development of UiO-66 by comparing its 
relative crystallinity and kinetic evolution overtime under different stirring times for $6 \mathrm{~h}$, $12 \mathrm{~h}, 24 \mathrm{~h}$, and $48 \mathrm{~h}$.

\subsubsection{Results and Discussion}

The primary purpose of this study was to test the hypothesis of the effect of stirring time on the morphology of UiO-66 by varying the stirring time of the reactants. Since most MOFs require $\leq 24$ hour stirring time, with UiO-66 being usually stirred for 24 hours, the following stirring times were chosen at $6,12,24$, and 48 hours to keep the study relevant with subsequent heating at $120^{\circ} \mathrm{C}$ for 24 hours via Scheme 1. The reactant concentrations and reaction conditions are summarized in Table 4.2.2-1. The as-synthesized sample was the $24 \mathrm{~h}$ stirred sample, being the benchmark for comparison and is bolded for reference. The best stirring time was chosen based on the phase purity, crystallinity and uniformity of crystals.

Table 4.2.2-1: Reactant composition and reaction conditions to investigate the effect of stirring time.

\begin{tabular}{ccccccc}
\hline $\begin{array}{c}\text { ZrCl4 } \\
\text { (mmol) }\end{array}$ & $\begin{array}{c}\text { 1,4-BDC } \\
\text { (mmol) }\end{array}$ & $\begin{array}{c}\text { DMF } \\
\text { (eq.) }\end{array}$ & $\begin{array}{c}\text { Acetic } \\
\text { Acid } \\
\text { (eq.) }\end{array}$ & $\begin{array}{c}\text { Stirring } \\
\text { time (h) }\end{array}$ & $\begin{array}{c}\text { Temperature } \\
\text { ( }{ }^{\circ} \text { C) }\end{array}$ & $\begin{array}{c}\text { Reaction } \\
\text { time (h) }\end{array}$ \\
\hline 0.343 & 0.343 & 993 & 30 & 6 & 120 & 24 \\
$\mathbf{0 . 3 4 3}$ & $\mathbf{0 . 3 4 3}$ & $\mathbf{9 9 3}$ & $\mathbf{3 0}$ & $\mathbf{1 2}$ & $\mathbf{1 2 0}$ & $\mathbf{2 4}$ \\
0.343 & 0.343 & 993 & 30 & 24 & 120 & 24 \\
0.343 & 0.343 & 993 & 30 & 48 & 120 & 24 \\
\hline
\end{tabular}

The formation and growth of UiO-66 samples were confirmed by the SEM images. As depicted in Figure 4.2.2-1, the 6h stirring time showed non-uniform ill-defined agglomerations of size $0.12 \pm 0.046 \mu \mathrm{m}$, while $12 \mathrm{~h}$ stirring time revealed clustered and intergrown crystals with an average diameter of $0.11 \pm 0.053 \mu \mathrm{m}$; however, the latter SEM image showed more faceted crystals. This trend was observed for the first time, that with a 
stirring time $>6$ hours, the particles start to disintegrate or decrease in crystallinity. At $24 \mathrm{~h}$ stirring time, the crystals appeared more well defined with rounded edges, and were mostly monodisperse and discrete in nature with an average diameter of $0.17 \pm 0.026 \mu \mathrm{m}$. The $48 \mathrm{~h}$ stirring time showed no agglomerations or definitive structures as such; However, the surface contains tiny ill-defined particles. Its morphology appeared as a large solid with grain-like assemblies throughout its surface. No distinct crystals were detected even at higher magnification.

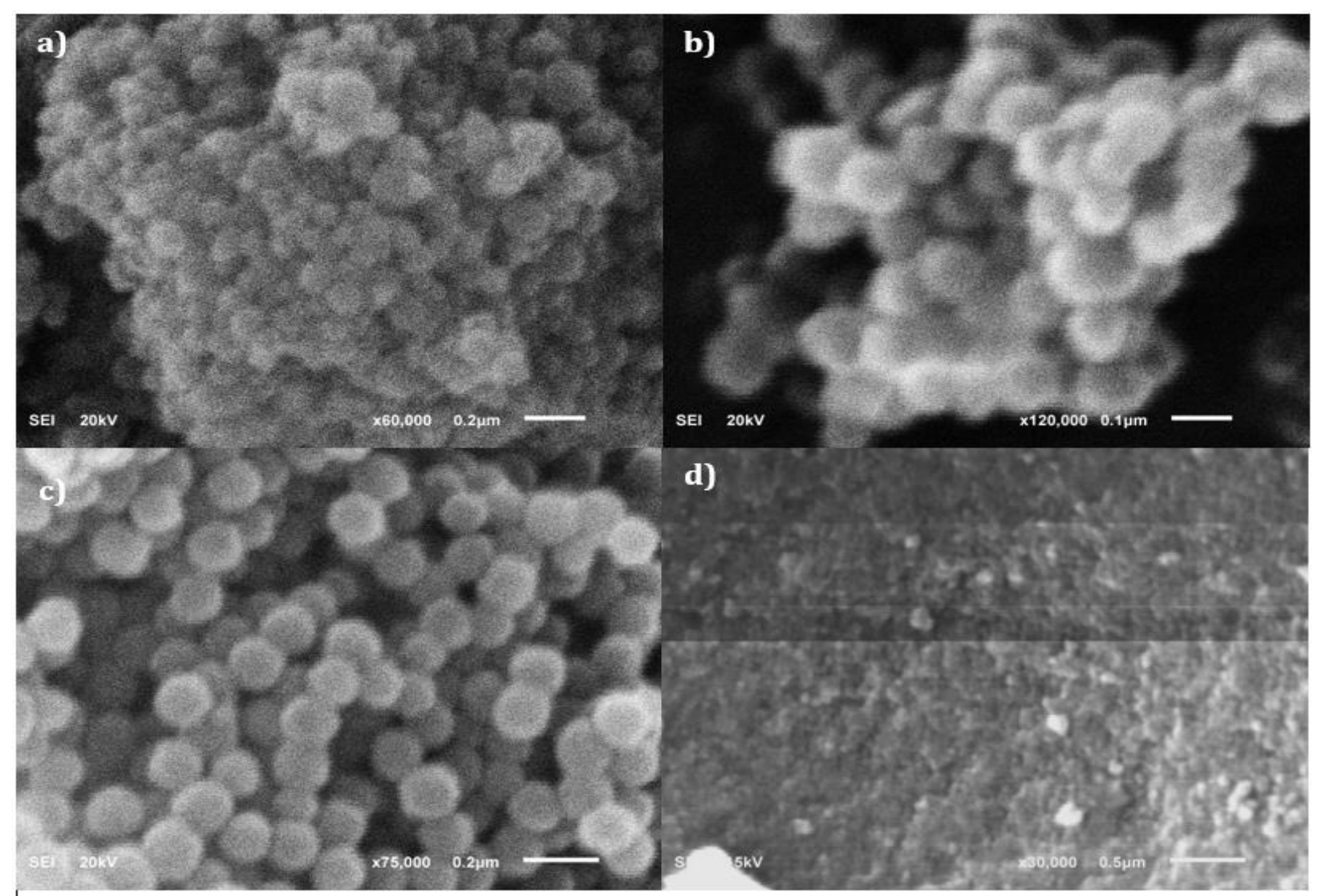

Figure 4.2.2-1: SEM images of UiO-66 with stirring times at a) 6h, b) $12 \mathrm{~h}$, c) $24 \mathrm{~h}$, and d) $48 \mathrm{~h}$.

Figure 4.2.2-2 elucidates the structural alteration of UiO-66 with the change in stirring time. The characteristic peak for UiO-66 at a $2 \theta$ angle of $7.28^{\circ}$, corresponding to the plane (111), showed highest peak intensity for 24-hour sample; while these peaks remained relatively low in intensity for all other samples. The 24-hour sample diffraction pattern was sharp, narrow and contained highest intensity peaks with no peak visible broadening or splitting. This indicated the formation of highly crystalline structures. The PXRD of the samples show no new peaks in comparison to the as-synthesized Ui0-66. The characteristic peak for the $6 \mathrm{~h}$ 
and $12 \mathrm{~h}$ samples at a $2 \theta$ angle of $7.28^{\circ}$, corresponding to the plane (111), expressed similar peak widths with reference to the as-synthesized sample; however, these samples consisted of visibly merged peaks corresponding to $25.42^{\circ}$ and $26.04^{\circ} 2 \theta$ angles. This was attributed to the peak broadening that merged peaks due to proximity. The PXRD pattern for $6 \mathrm{~h}$ stirring also marked the presence of relatively broad peaks and low intensity indicating small particle size. Although the intensities of the diffractogram for all samples remained constant at higher angles in comparison to the as-synthesized sample, the $12 \mathrm{~h}$ sample yielded better crystallinity than the $6 \mathrm{~h}$ sample. Further increase in stirring time beyond $24 \mathrm{~h}$, i.e., 48 hours dropped the intensity drastically, producing a relatively flat diffraction pattern over the entire $2 \theta$ range. The reduced intensity and flattened peaks indicate drastic decrease in particle size along with very poor crystallinity.

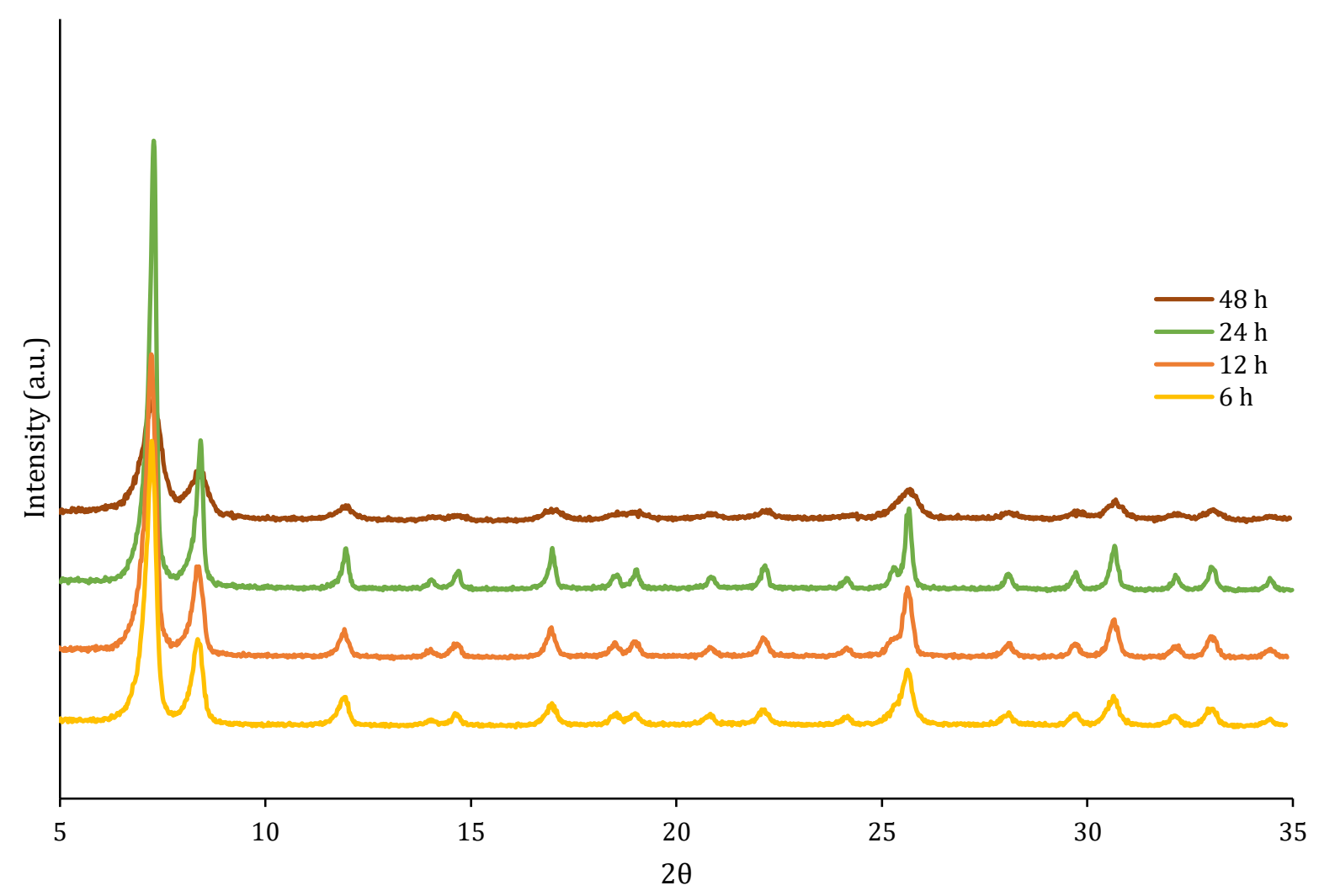

Figure 4.2.2-2: XRD patterns on varying stirring times for $6 \mathrm{~h}, 12 \mathrm{~h}, 24 \mathrm{~h}$, and $48 \mathrm{~h}$.

The crystallinities of all the sample were calculated by taking the ratio of the intensities of characteristic peak of the individual PXRD patterns at $7.64^{\circ}$ to a relatively constant peak for 
all samples, i.e. at $34.68^{\circ}$. The calculated crystallinities were normalized for all the samples and compared to the crystallinity of the as-synthesized sample. The results were consolidated as shown in Figure 4.2.2-3.

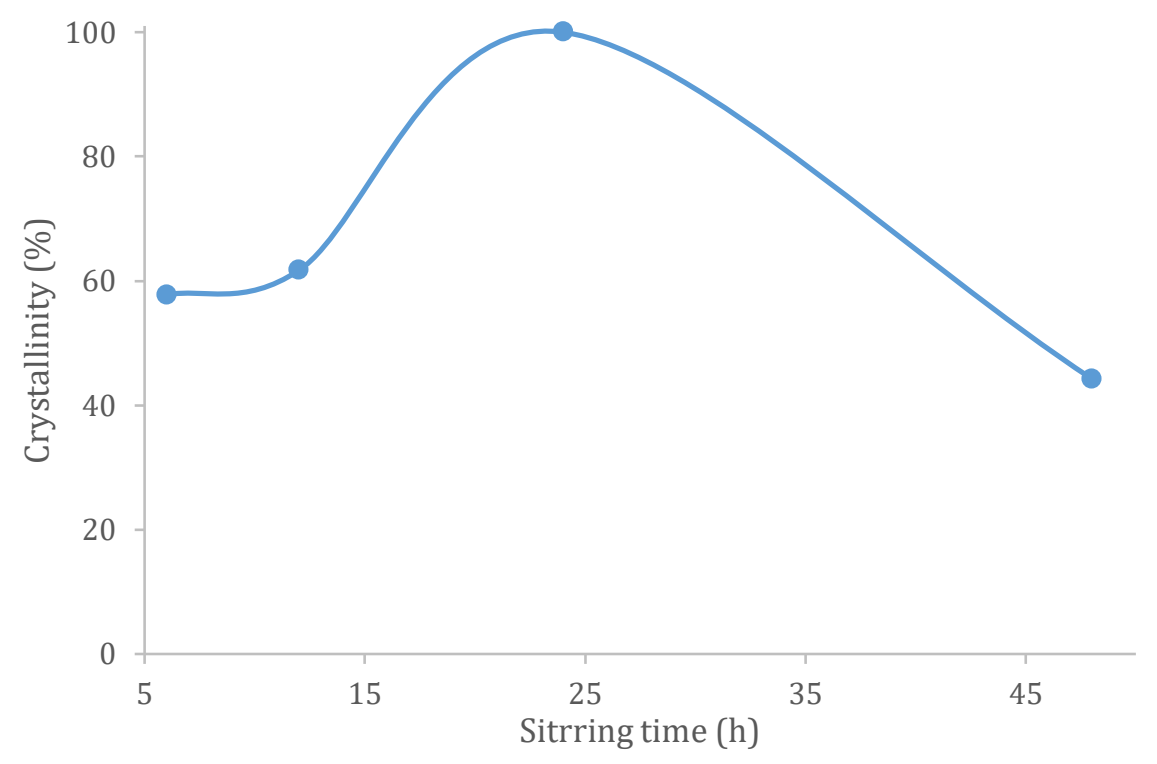

Figure 4.2.2-3: Effect of stirring time on the crystallinity of UiO-66 crystals.

From Figure 4.2.2-3, a comparison of the crystallinities of UiO-66 crystals for varied stirring time to the as-synthesized revealed that Ui0-66 particles were generated within the first 6 hours of stirring. However, the diffractogram suggested that the UiO-66 particles had been formed with poor crystallinity. These conclusions were also drawn from the SEM results, which indicated round ill-defined, clustered growth with $6 \mathrm{~h}$ stirring. When the stirring time was increased past $6 \mathrm{~h}$, up to $12 \mathrm{~h}$, the SEM indicated better crystallinity of the particles, which was also indicated by the XRD pattern. With increase in stirring time to $24 \mathrm{~h}$, fully developed particles with highest crystalline Ui0-66 particles were achieved, after which the crystallinity dropped drastically.

Many zeolites, analogous to MOFs, and minerals have also demonstrated a significant impact of stirring time on its morphology [84], [85]. The high crystallinity UiO-66 obtained for $24 \mathrm{~h}$ stirring time can be justified by the growth pattern identified for ZIF-8 [85] in which stirring time was recognized as an essential factor in initiating the nucleation process by increasing the number of collisions. The smaller solute particles contained unsatisfied bonds due to 
which the molecules readily diffused into the solution and lodged on the surface of large particles to grow by a mechanism known as Ostwald ripening. A similar observation was made by Cheney et al. [85] in the case of Birnessite nanoparticles where aging of the reactant mixture imposed homogeneous nucleation allowing uniform growth of nanofibers. The apparent growth mechanism was reported in the following order where the nucleation is initiated by the frequent collision of molecules, whereupon nucleation occurs on the surface of the nanoparticles. These linkages were extended through permanent aggregation of smaller molecules on the surface of larger molecules (Ostwald ripening). The proposed mechanism was also rationalized by Gibbs free energy where the reaction proceeded to minimize the energy between the nucleus and the substrate. Furthermore, another study reported that appropriate aging time stabilizes zeolite nuclei in precursor solution which in turn decreases the reaction energy barrier in the crystallization process which promotes formation of specific pure phase crystals at lower temperature and shorter reaction time [86]. The change in the morphology on increasing the stirring time from intermediate phase to pure phase UiO-66 crystals stems from the labile regions of the $\mathrm{Zr}-\mathrm{O}$ linkages that enable the entity to undergo structural changes. At short stirring times, i.e. $6 \mathrm{~h}$ and $12 \mathrm{~h}$, the amount of dissolved particles was lower which made it difficult to achieve the necessary molecular integrations and form homogeneous particles. As a result, the particles were agglomerated and polydisperse in nature. When the stirring time was increased to $24 \mathrm{~h}$, the solute particles experienced longer time for collisions that led to dissolutions and higher particle integration. This resulted in UiO-66 crystals being more homogeneous, structured, and crystalline. On the other hand, extended stirring time, up to $48 \mathrm{~h}$, increased particle dissolutions that stabilized numerous nuclei within the solution. However, the prolonged stirring caused the stabilized nuclei to break down due to collisions and attritions. This increased the density of small Ui0-66 particles, thus delivering subpar crystallinity.

\subsubsection{Conclusion}

This work demonstrated the hypothesized influence of stirring time on the morphology of UiO-66 for the first time by using varying stirring times of $6 \mathrm{~h}, 12 \mathrm{~h}, 24 \mathrm{~h}$ and $48 \mathrm{~h}$. solutionbased synthesis of UiO-66 solely at room temperature. It was evident that stirring time is an important factor in changing the morphology of UiO-66. The PXRD and SEM results are 
consistent with the findings in literature. Although all stirring times successfully produced UiO-66, the crystallinity of all samples were substandard compared to the 24-hour sample. Despite being able to form within $6 \mathrm{~h}$ stirring, the parent UiO-66 is significantly lower in crystallinity than the $24 \mathrm{~h}$ sample. The stirring time of $6 \mathrm{~h}$ and $12 \mathrm{~h}$ can be considered insufficient to produce enough nuclei that keeps the energy barrier of the crystallization reaction significantly high to produce crystalline UiO-66 particles. Increased stirring up to $24 \mathrm{~h}$ ensures solute breakdown, appropriate collision of particles to produce precursor nuclei that become the precursor of growth for Ui0-66. This interaction allows primary nucleation on the surface of the particles [85]. The pre-formed stable nuclei decrease the energy barrier of the reaction giving them [86] a better chance to further grow into larger particles through aggregation [85] when exposed to higher temperature. Therefore, it is safe to suggest that 24 hours of stirring is enough to provide appropriate amount of nucleation, molecular integration and interaction that results in highly crystalline UiO-66. However, concrete conclusions can be drawn using in depth analysis of in-situ PXRD and SAED (selected-area electron diffraction) results for stirring times below 6 hours to confirm initial nucleation period. Based on the results on this section, the next experiments were carried out with 24 hour stirring time.

\subsection{EFFECT OF REACTION TIME}

\subsubsection{Introduction}

Scarce investigations were conducted to study the effect of reaction time on the growth of MOF crystals. Reports indicate that the solvothermal synthesis time for most microporous materials is usually 24 hours in order to produce pure phase crystalline materials. An increase in the reaction time up to $24 \mathrm{~h}$ enhances monodispersity and size of crystals, beyond which both features start to degenerate [87], [88]. The structural evolution of these materials experiences a stage of initial nucleation following fully developed crystals at published synthesis time [76], [87], [88]. Therefore, most studies limit the reaction time to the fully developed crystal stage. 
In the case of MOFs, a strong correlation of the stability of MOF was found with the reaction time where time resolved PXRD experiments confirmed that prolonged reaction time irreversibly destroyed the MOF-14's framework, forming crystalline $\mathrm{Cu}_{2} \mathrm{O}$ as the sole product [89]. A similar study by Mulyati et al. [88] also revealed the presence of an optimum time and temperature in the synthesis of MOF-5 under $105^{\circ} \mathrm{C}$ for $144 \mathrm{~h}, 120^{\circ} \mathrm{C}$ for $24 \mathrm{~h}$ or $140^{\circ} \mathrm{C}$ for $12 \mathrm{~h}$. These specific conditions were chosen based on the diffraction patterns that followed the highest crystallinity simulated pattern of MOF-5. The investigation was performed by calculating the area of 4 major representative peaks in the diffractogram with largest area indicating 100\% crystallinity. A comparison of the SEM images of the three reaction conditions showed cuboidal crystals of 85-95 $\mu \mathrm{m}, 250-300 \mu \mathrm{m}$, and 100-110 $\mu \mathrm{m}$ for $144 \mathrm{~h}, 24 \mathrm{~h}$, and $12 \mathrm{~h}$, respectively, which suggested that increasing the reaction time and temperature increases the size of the crystals but only up to an extent. Beyond that mark, the crystallinity starts to drop. Both SEM images and XRD patterns are in agreement, with the highest crystallinity observed for $120^{\circ} \mathrm{C}$ for $24 \mathrm{~h}$ synthesis condition as the optimum condition to produce MOF-5 crystals.

For zeolites, some crystals can undergo morphological change and appear dissimilar in structure than the originally synthesized zeolites with extended reaction time by selectively changing their structural units [90]-[92]. On the other hand, MOFs being very similar in nature to zeolites have not reported this strange reorientation. Additionally, many studies have reported that increasing the aging time of the precursor solution resulted in pure phase crystals [86] and shortened the crystallization time [93]. Therefore, the next initiative of this project was to study the growth of UiO-66 crystals over the chosen reaction times of $12 \mathrm{~h}$, $24 \mathrm{~h}, 48 \mathrm{~h}$, and $72 \mathrm{~h}$ by stirring for $48 \mathrm{hours}$ and heating to $120^{\circ} \mathrm{C}$ for the stated reaction times. The stimulating observations confirmed successful reorientation of crystals to the originally synthesized octahedral structures at prolonged reaction time. Additionally, it was also noted that extending the stirring time was capable of scaling down the crystallization time required for manufacturing the UiO-66 crystals. 


\subsubsection{Results and Discussion}

The primary purpose of this study was to detect any change in morphological features of UiO-66 crystals with $48 \mathrm{~h}$ stirring time for prolonged reaction times from $12 \mathrm{~h}, 24 \mathrm{~h}, 48 \mathrm{~h}$, and $72 \mathrm{~h}$ and subjected to $120^{\circ} \mathrm{C}$ reaction temperature via Scheme 1. These reaction times were chosen based on the published synthesis time of 24 hours for applicability of the study. Although $24 \mathrm{~h}$ stirring was concluded the best choice for producing highest crystalline particles, the stirring time was intentionally increased to $48 \mathrm{~h}$ under the hypothesis that prolonged stirring time would cut down the crystallization time, as observed in zeolite crystals [93]. The reactant concentrations and reaction conditions are summarized in Table 4.3.2-1. The as-synthesized sample was stirred for $24 \mathrm{~h}$ and heated to $120^{\circ} \mathrm{C}$ for $24 \mathrm{~h}$, was the benchmark for comparing all other samples and is bolded for reference. The best reaction time was chosen based on the phase purity, crystallinity and uniformity of crystals.

Table 4.3.2-1: Reactant composition and reaction conditions to investigate the effect of reaction time.

\begin{tabular}{ccccccc}
\hline $\begin{array}{c}\text { ZrCl4 } \\
(\mathbf{m m o l})\end{array}$ & $\begin{array}{c}\mathbf{1 , 4 - B D C} \\
(\mathbf{m m o l})\end{array}$ & $\begin{array}{c}\text { DMF } \\
\text { (eq.) }\end{array}$ & $\begin{array}{c}\text { Acetic } \\
\text { Acid } \\
\text { (eq.) }\end{array}$ & $\begin{array}{c}\text { Stirring } \\
\text { time (h) }\end{array}$ & $\begin{array}{c}\text { Temperature } \\
\left({ }^{\circ} \mathbf{C}\right)\end{array}$ & $\begin{array}{c}\text { Reaction } \\
\text { time (h) }\end{array}$ \\
\hline 0.08 & 0.057 & 993 & 30 & 48 & 120 & 12 \\
$\mathbf{0 . 3 4 3}$ & $\mathbf{0 . 3 4 3}$ & $\mathbf{9 9 3}$ & $\mathbf{3 0}$ & $\mathbf{1 2}$ & $\mathbf{1 2 0}$ & $\mathbf{2 4}$ \\
0.08 & 0.057 & 993 & 30 & 48 & 120 & 24 \\
0.08 & 0.057 & 993 & 30 & 48 & 120 & 48 \\
0.08 & 0.057 & 993 & 30 & 48 & 120 & 72 \\
\hline
\end{tabular}

As shown in Figure 4.3.2-1, the PXRD patterns were obtained for each of the samples and compared to the as-synthesized sample. Bearing in mind that the samples were stirred for $48 \mathrm{~h}$, the first apparent observation from the diffraction pattern was that UiO-66 crystals were readily synthesized with a short reaction time of $12 \mathrm{~h}$ reaction. On observing the 
diffraction patterns of the 4 stated samples over the $2 \theta$ range $\left(5-35^{\circ}\right)$, the $12 \mathrm{~h}$ sample produced intense and sharp peaks in comparison to the synthesize sample, however, its intensity was lower. The diffraction pattern began to weaken and broaden after $12 \mathrm{~h}$ reaction time but seemed to intensify and narrow down when the reaction approached $72 \mathrm{~h}$. It was noted that the PXRD patterns of $72 \mathrm{~h}$ and as-synthesized sample resembled in appearance but was much lower in intensity.

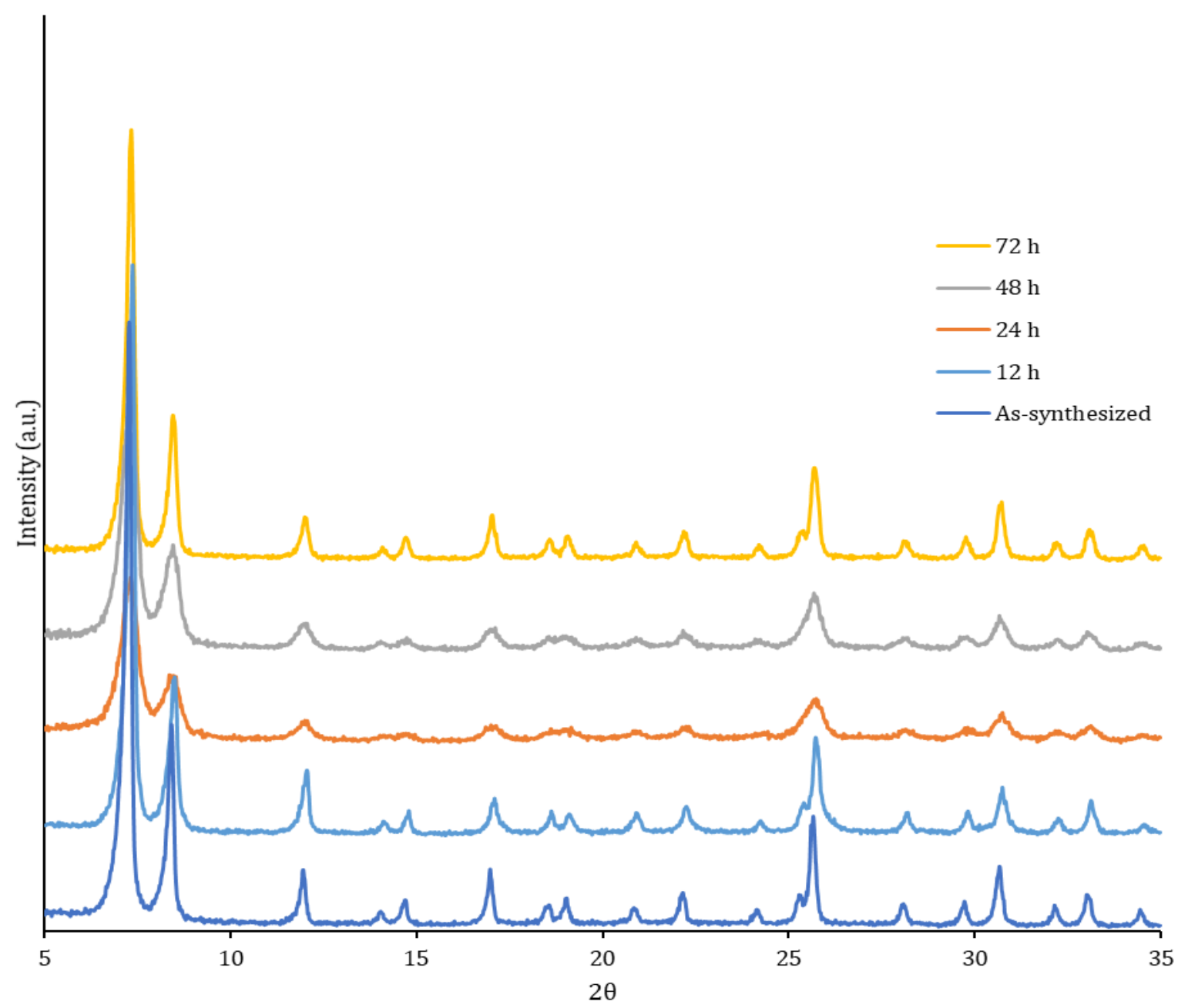

Figure 4.3.2-1: PXRD patterns on varying reaction times after 48 hours of stirring.

In reference to the SEM images in Figure 4.3.2-2, it was apparent that the crystals produced after $12 \mathrm{~h}$ reaction time are intergrown and polydisperse in nature. The images for $24 \mathrm{~h}$ reaction time showed the presence of a large solid mass with minute grain-like intergrown crystals which were undistinguishable. Upon increasing the reaction time to $48 \mathrm{~h}$, the crystals 
became distinguishable with clustered appearance of very small, ill-defined particles but remained intergrown. When the reaction time was further increased to $72 \mathrm{~h}$ reaction, the crystals were smaller in comparison to the as-synthesized sample but remained agglomerated and polydisperse in form.

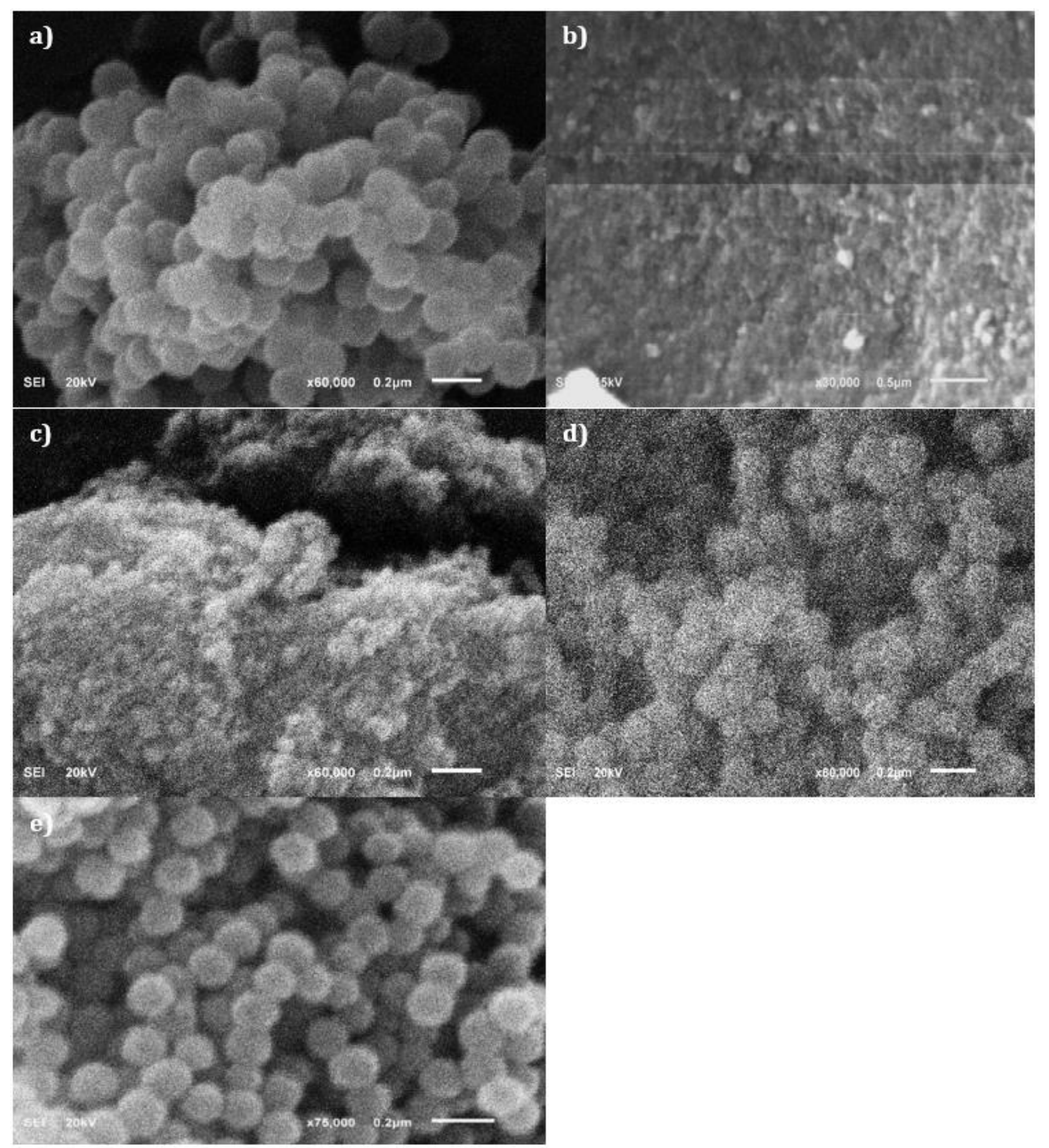

Figure 4.3.2-2: SEM images of Ui0-66 with reaction times after $48 \mathrm{~h}$ of stirring at a) $12 \mathrm{~h}, \mathrm{~b}$ ) $24 \mathrm{~h}, \mathrm{c}) 48 \mathrm{~h}, \mathrm{~d}) 72 \mathrm{~h}$, and e) as-synthesized. 
The crystallinities were calculated by the peak ratio method introduced in Section 4.2.2. The results were consolidated by comparing the crystallinities of the samples to the assynthesized sample and are represented in Figure 4.3.2-3.

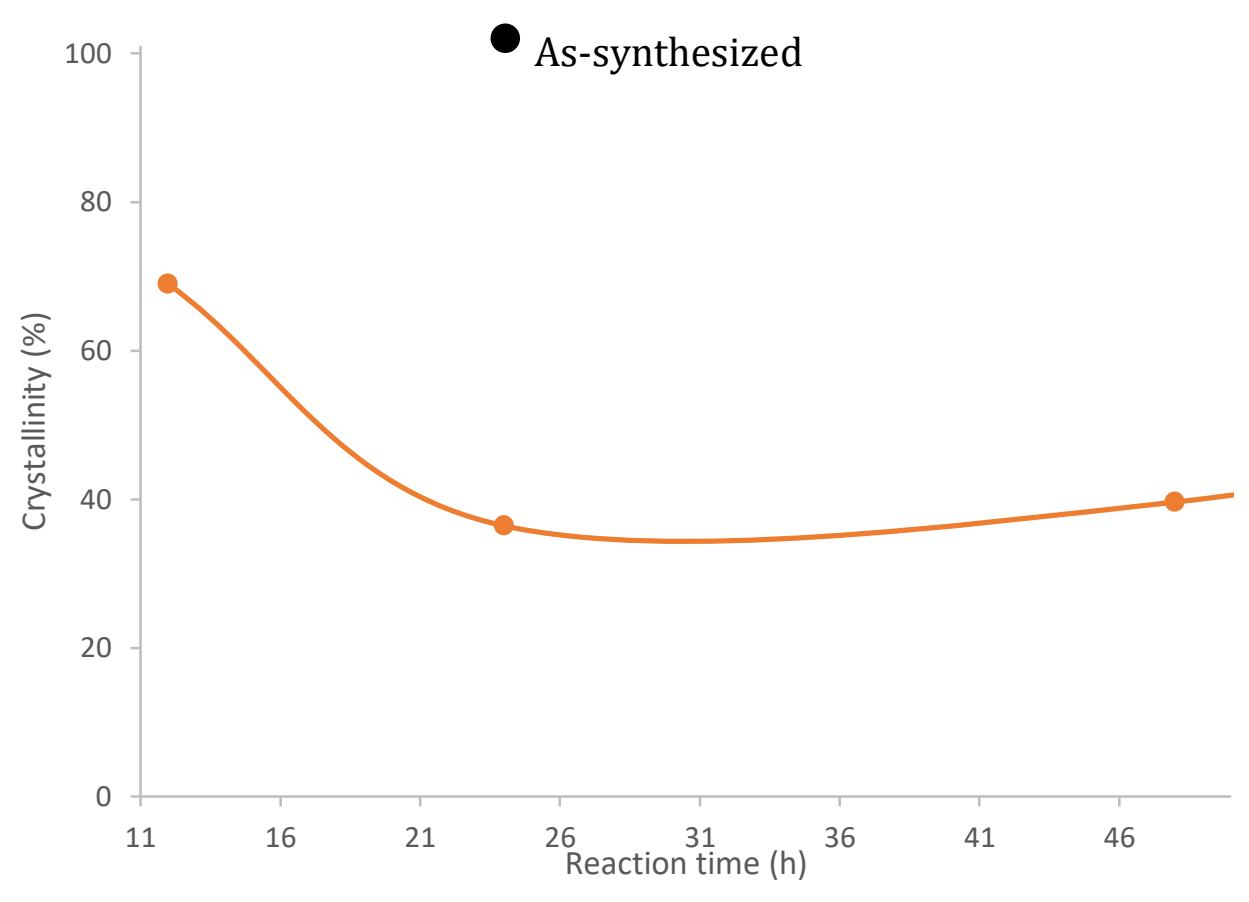

Figure 4.3.2-3: Effect of reaction time and extended stirring time on the crystallinity of UiO66.

The first observation made from Figure 4.3.2-3 was that using a longer stirring of $48 \mathrm{~h}$ shrunk the time required for the crystallization stage where UiO-66 crystals as opposed to 24 hours. The PXRD and SEM results revealed equivalent crystallinity within a $12 \mathrm{~h}$ reaction time compared to as-synthesized product. This observation is similar to the results drawn by Okubo et al. [93] in the crystallization of Faujasite (FAU) zeolite crystals, whereupon lengthening the aging time of the crystals salvaged the crystallization time of the reactants producing crystalline materials. The quantitative analysis of liquid phase of the reactants confirmed that the hydrogel contained aluminosilicate species specifically formed in the aging process produced FAU crystals with high crystallinity and sharp size distribution. The SEM images of the FAU zeolite also attested to the conclusion where with 2-days of aging period reduced the crystallization time to $6 \mathrm{~h}$, while a minimum of $3 \mathrm{~h}$ was required for producing FAU with 7-day aging period with the formation of by-product phases of sodalite 
(SOD), chabazite (CHA), and analcime (ANA). The conclusion was attributed to the structural reorganizations that occurred during the aging period where the aluminosilicate precipitate aids the formation of zeolitic precursors by a mechanism called the Ostwald's step rule of successive transformations, which in turn accelerated the crystallization rate of FAU.

In addition, another peculiar observation through Figure 4.3.2-3 was the re-crystallization growth pattern. The PXRD and SEM results revealed that UiO-66 particles grew within $12 \mathrm{~h}$ reaction time (parent MOF) with a stirring time was of $48 \mathrm{~h}$ with comparable crystallinity as the as-synthesized sample. However, when the reaction time was increased to $48 \mathrm{~h}$, the crystals had started to reform and gained significant crystallinity by $72 \mathrm{~h}$ reaction time. This phenomenon could be related to the ADOR mechanism occurring in zeolites where extending zeolite crystals (parent) are selectively modified to form a different phase of crystalline zeolites [94]. Contrast to individually altering the ADOR mechanism in zeolites, UiO-66 seems to undergo an intrinsic ADOR mechanism with extending reaction time. Thus, this phenomenon could be related to zeolites as follows - the UiO-66 crystals synthesized at $12 \mathrm{~h}$ marked the initial formation of parent UiO-66 which is the assembling process. The increased stirring time may have produced sufficient nucleation/crystallization to form highly crystalline particles. On further increase in reaction time to $24 \mathrm{~h}$, the crystallinity dropped which was also consistent with the SEM information. This may have been due to the increased content of UiO-66 crystals in which led them to re-dissolve in the solution. This was referred to as the dis-assembling process. When the reaction time was increased to $48 \mathrm{~h}$, the particles seemed to start re-assembling themselves producing a substandard version of UiO-66 which, at $72 \mathrm{~h}$ mark, presented itself as UiO-66 crystals with significantly better crystallinity. As perceived in this discourse, the process appears to be parallel to the envisioned ADOR process in zeolites except that in UiO-66, it undergoes this process intrinsically. Although the UiO-66 crystals were developed from the parent MOF, the parent MOF produced the highest crystallinity MOF among other samples but did not outperform the as-synthesized sample. However, it was noted that the crystallinity of the parent MOF was lower than the as-synthesized sample. In addition, the same naturalistic ADOR mechanism was observed for the extended reaction time even at a temperature of $140^{\circ} \mathrm{C}$. The results are included in the Appendix (Figure A.1-1 and Figure A.1-2). 
Similar solid-state rearrangement has also been observations in MOFs such as the transformation of MOF-235 to MIL-53 for MIL-53(Fe) [99] and MIL-101(V) and MIL-88B(V) will convert to MIL-47 [95]. In this process, the structures rose from the same reaction system under specific system environments from intermediate phases through thermodynamically driven dissolutions and recrystallizations. The intermediate phases are unstable phases which undergo rapid solid-state rearrangement in the Co-BDC-DMF mixture. The difference in formations is due to the various coordination modes of Co metal atom that permits the interatomic modifications during synthesis. The coordination changed was a topotactic arrangement of Co atoms that changed from triple octahedral to tetrahedral-octahedral-tetrahedral framework arrangement.

This behavior is a contrast to the cited MOFs as observed particles assemble, then disintegrate to reassemble from the parent UiO-66 while maintaining their structural integrity throughout the process. Moreover, as this phenomenon has been observed for the first time in UiO-66 particles, the only phenomenon it can be related to is the thermodynamically driven ADOR mechanism. The increased reaction time in the solvothermal synthesis process, influences its nucleation and/ or crystallization rates, causing the nuclei to grow. The prolonged reaction time changes the dynamics of the system going from supersaturation of solution to supersaturation of the UiO-66 crystals itself that cause dissolutions to form highly crystalline UiO-66 from parent UiO-66 MOF.

\subsubsection{Conclusion}

This experiment demonstrated the impact of reaction time on the morphology of UiO-66 by increasing the stirring time to $48 \mathrm{~h}$ and varying the reaction time at $12 \mathrm{~h}, 24 \mathrm{~h}, 48 \mathrm{~h}$, and $72 \mathrm{~h}$ at $120^{\circ} \mathrm{C}$. To summarize, for the first time, a reorientation of structure into its parent UiO-66 was observed upon prolonging the reaction time beyond $24 \mathrm{~h}$. The molecules first underwent crystallization until a $12 \mathrm{~h}$ reaction time with highest Bragg peaks observed for this sample among other reaction times, indicating highest crystallinity. The crystals then reoriented for the second time at $72 \mathrm{~h}$ after undergoing dissolutions past $12 \mathrm{~h}$ reaction time. Although, the samples reformed to produce UiO-66 at 72h, the diffraction pattern and SEM indicated lower 
crystallinity and poorly defined entities than the parent MOF (as-synthesized UiO-66). Thus, as per the observation, the structural reorganization of UiO-66 over an extended reaction period may be a thermodynamically driven ADOR process.

\subsection{EFFECT OF MOLAR RATIO OF Zr:BDC}

\subsubsection{Introduction}

An appropriate combination of metal and linker forming the SBU is necessary for building a MOF with large surface areas. This has driven substantial efforts among researchers to develop MOFs with different dimensionality and composite framework by adjusting the metal:linker ratio [96], [97]. Most solvothermal syntheses adopt a 1:1 ratio of metal to ligand while a handful of researchers explored the effect of this factor on its framework. For example, Yin et al. [96] also demonstrated the consequences of changing molar ratio of reactants on the morphology of Cd-MOFs with1,2,4 triazole ligand (TAZ) linker. Keeping all other reaction variables constant, a metal:linker ratio of 1:1.2 produced a 3-dimensional network of $\left\{\left[\mathrm{Cd}_{3} \mathrm{Cl}_{3}(\mathrm{TAZ})_{3}(\mathrm{DMF})_{2}\right]_{\mathrm{n}}\right\}$ with a TAZ coordination number of 3 (Figure 4.4.1-1a); while a metal:linker ratio of 1:1.7 produced 1D structure of $\left\{\left[\mathrm{CdCl}_{2}(\mathrm{TAZ})\right]_{\mathrm{n}} \mathrm{n}\left(\mathrm{H}_{2} \mathrm{O}\right)\right\}$ with TAZ coordination number of 1 (Figure 4.4.1-1b) [96].

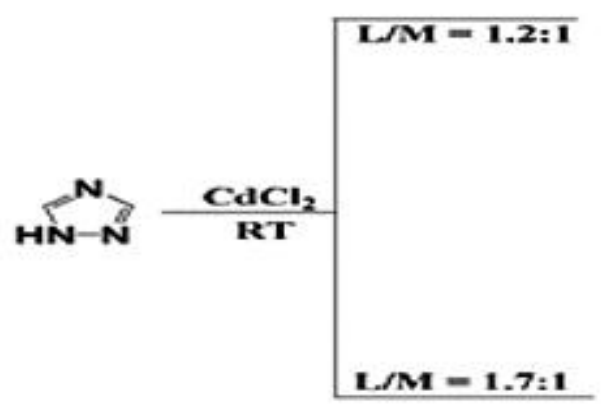

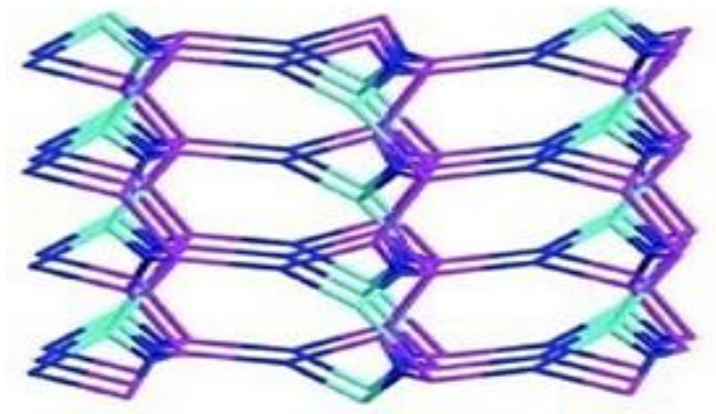

a)

$1 \mathrm{D}$ coordination polymer

b)

Figure 4.4.1-1: Schematic representation of effect of change in metal:linker concentration [96]. 
Likewise, Wu et al. [97] observed the change in dimensionality of Zn compounds obtained from the solvothermal synthesis of zinc salts and 4,4'-(hexafluoroisopropylidene) bis(benzoic acid) ( $\mathrm{H}_{2}$ hfipbb) and 4,4'-dipyridylsulphide (dps) as a co-ligand. By altering the metal:linker:co-ligand ratio, three distinct topologies were obtained. A 1:1:0.1 ratio produced $\left[\mathrm{Zn}(\mathrm{hfipbb})\left(\mathrm{H}_{2} \mathrm{hfipbb}\right)_{0.5}\right]_{\mathrm{n}}$, a 3D two-fold interpenetrable framework (Figure 4.4.1-2 a); while other co-ligand concentrations generated non-interpenetrable and two-fold penetrable 3D bimodal-connected framework isomers of the formula [ $\mathrm{Zn}_{2}$ (hfipbb) $\left.{ }_{2}(\mathrm{dps})\left(\mathrm{H}_{2} \mathrm{O}\right)\right]_{\mathrm{n}}$ (Figure 4.4.1-2 b) and c) [ [97].

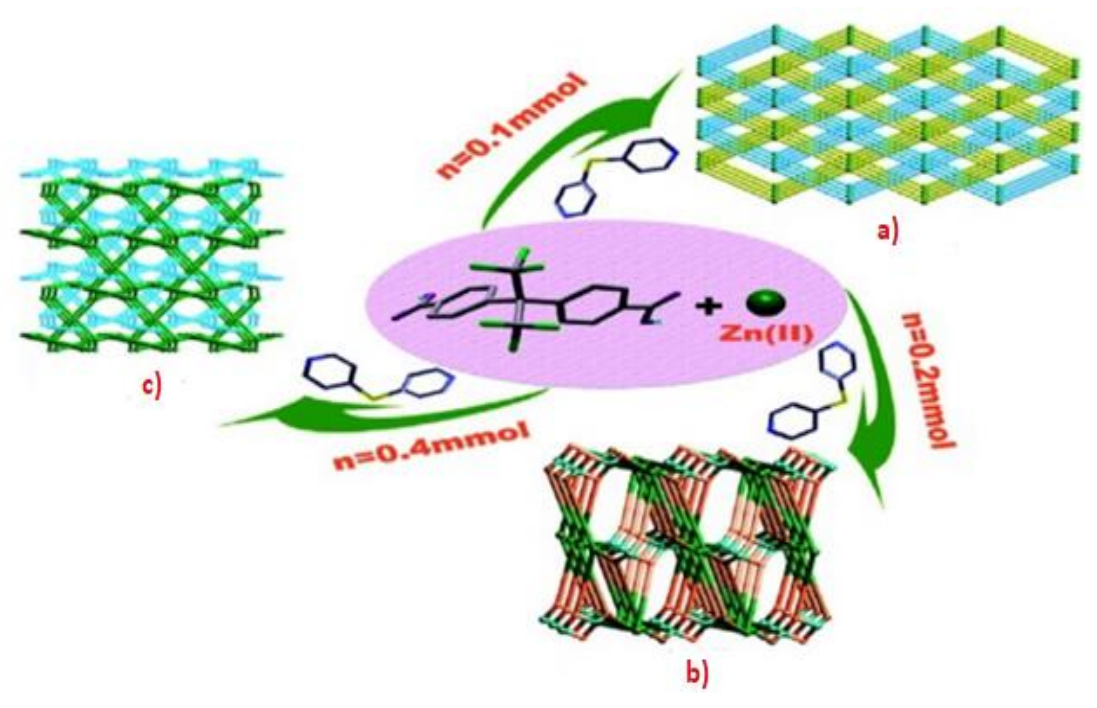

Figure 4.4.1-2: Distinct frameworks synthesized from Zn-based MOF with varying co-ligand concentration of a) $0.1 \mathrm{mmol}, \mathrm{b}$ ) $0.2 \mathrm{mmol}$, and c) $0.4 \mathrm{mmol}$ [97].

For UiO-66 in particular, Zou et al. [98], inspired by the popular solvent-free synthesis of zeolites, has developed an innovative method of producing UiO-66 in a template mediated environment, highlighting the effects of alteration of the Zr:BDC ratio on its properties. However, the highlighted outcomes in this study referred to template mediated synthesis specifically while solvothermal syntheses are sensitive to the type of method employed in production of the MOF. Therefore, these findings urged us to study the effect of adjusting the molar ratio of the metal and linkers on the morphology of UiO-66 under solvothermal synthesis conditions. However, in this case, the concentrations of the reactants were adjusted to a new formula that developed seemingly perfect octahedral monodisperse crystals with a 1:1 Zr:BDC ratio to accurately determine the impact of changing metal:linker 
ratio. Accordingly, based on the results from the preliminary experiments and modified concentrations [72] was adopted and refined to Scheme 3. In this synthesis route, the molar ratio of $\mathrm{Zr}: \mathrm{BDC}$ was adjusted as 1:1 (as-synthesized), 1:1.5, 1:2, 1.5:1, and 2:1 without the addition of co-solvent.

\subsubsection{Results and Discussion}

The primary aim of this experiment was to investigate the effect of changing the metal to linker ratio on the morphology of UiO-66. The metal to linker amount was adjusted by the ratios 1:1.5, 1.5:1, 1:2, and 2:1 via Scheme 3. All other variables were kept constant and the mixture was stirred for $24 \mathrm{~h}$ followed by heating at $120^{\circ} \mathrm{C}$ for $24 \mathrm{~h}$. The reactant concentrations and reaction conditions are summarized in Table 4.4.2-1. The as-synthesized sample was also produced via Scheme 3 and stirred for $24 \mathrm{~h}$ with reaction time of $24 \mathrm{~h}$ at $120^{\circ} \mathrm{C}$ and is bolded for reference. The best molar ratio was chosen in comparison to the assynthesized sample based on the phase purity, crystallinity and uniformity of crystals.

Table 4.4.2-1: Reactant composition and reaction conditions to investigate the effect of metal:linker ratio.

\begin{tabular}{cccccccc}
\hline $\begin{array}{c}\text { Metal: } \\
\text { Linker }\end{array}$ & $\begin{array}{c}\text { Metal- } \\
\text { ZrCl4 } \\
\text { (mmol) }\end{array}$ & $\begin{array}{c}\text { Linker- } \\
\mathbf{1 , 4 - B D C} \\
\text { (mmol) }\end{array}$ & $\begin{array}{c}\text { DMF } \\
\text { (eq.) }\end{array}$ & $\begin{array}{c}\text { Acetic } \\
\text { Acid } \\
\text { (eq.) }\end{array}$ & $\begin{array}{c}\text { Stirring } \\
\text { time (h) }\end{array}$ & $\begin{array}{c}\text { Temperature } \\
\left({ }^{\circ} \mathbf{C}\right)\end{array}$ & $\begin{array}{c}\text { Reaction } \\
\text { time (h) }\end{array}$ \\
\hline $\mathbf{1 : 1}$ & $\mathbf{0 . 3 4 3}$ & $\mathbf{0 . 3 4 3}$ & $\mathbf{8 6 6}$ & $\mathbf{1 6 0}$ & $\mathbf{2 4}$ & $\mathbf{1 2 0}$ & $\mathbf{2 4}$ \\
$1: 1.5$ & 0.343 & 0.343 & 866 & 160 & 24 & 120 & 24 \\
$1.5: 1$ & 0.343 & 0.343 & 866 & 160 & 24 & 120 & 24 \\
$1: 2$ & 0.343 & 0.343 & 866 & 160 & 24 & 120 & 24 \\
$2: 1$ & 0.343 & 0.343 & 866 & 160 & 24 & 120 & 24 \\
\hline
\end{tabular}

The PXRD patterns for increasing the linker concentration are represented in Figure 4.4.2-1. The diffractogram of the as-synthesized was in good agreement with the literature (Figure 4.1.2-2). When compared to the as-synthesized material, increasing the concentration of the 
BDC linker to 1.5 times of zirconium, the intensity of the peaks at the $2 \theta$ angles, 7.7 and $8.5^{\circ}$ of the diffraction pattern decreased with no significant shifting or peak broadening of the pattern. This indicated that the sample exhibited no significant reduction in crystallinity. However, the intensities for the pattern at higher angles remained constant. Further increase in BDC linker up to 2 times showed even lower intensities and broader peaks than the 1.5 ratio of BDC linker, indicating further reduction in crystallinity.

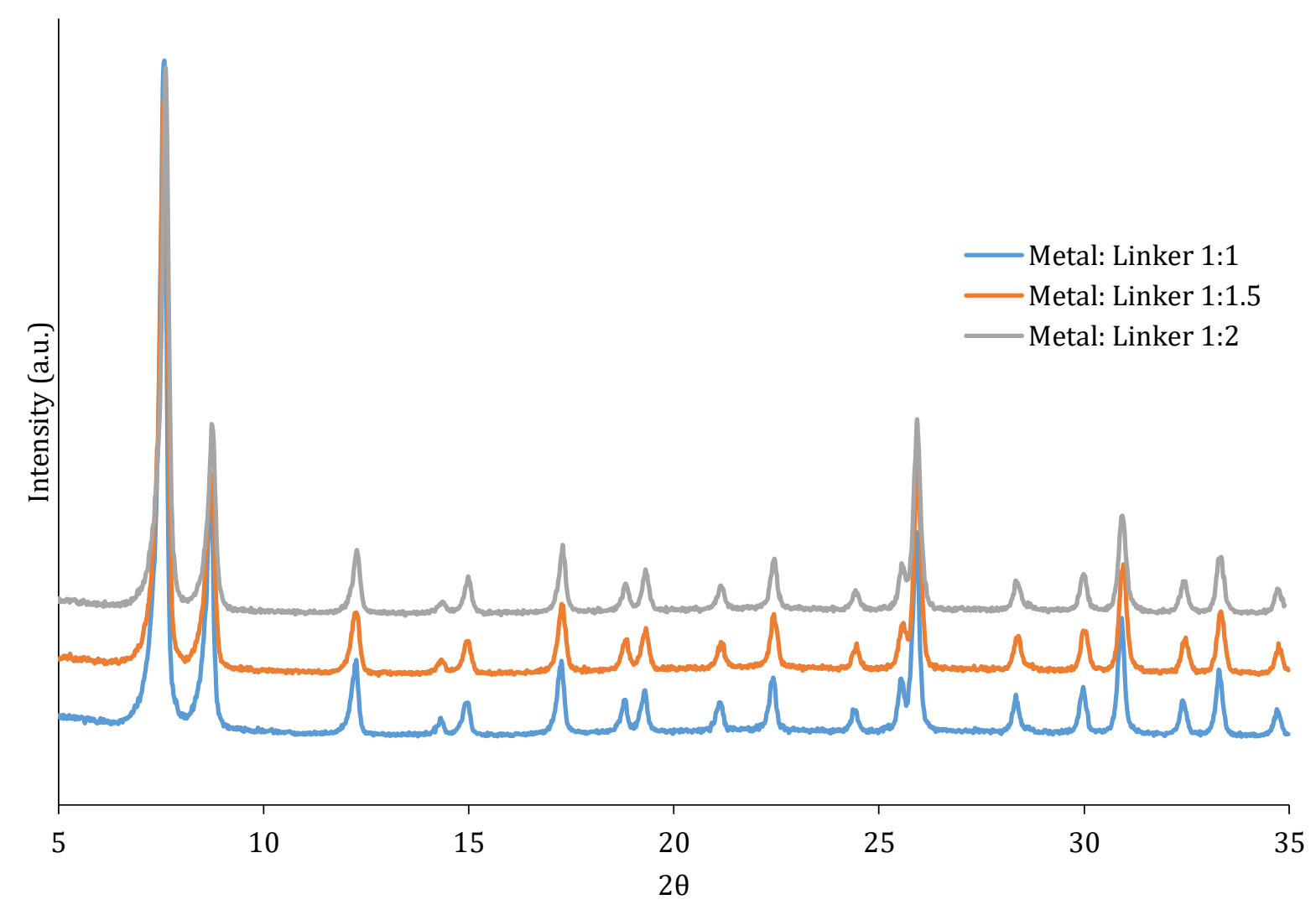

Figure 4.4.2-1: PXRD pattern of UiO-66 with $\mathrm{Zr}$ (metal) as a limiting reactant.

Regarding the SEM results, as seen in Figure 4.4.2-2, the crystals of the as-synthesized sample are slightly blunt edged, mostly monodisperse octahedral crystals of about $0.2 \mu \mathrm{m}$ in size. On increasing the BDC linker amount to 1.5 times, the particles appeared bigger than the as-synthesized crystals. The particles grew to $0.516 \pm 0.0151 \mu \mathrm{m}$. Using higher BDC content in the precursor solution also yielded particles showing truncated edges with tumor like growth on some facets with sizes up to $0.5 \mu \mathrm{m}$. Upon increasing the concentration further up to 2 times, the particles reduced in size to $0.453 \pm 0.0149 \mu \mathrm{m}$. The tumor like 
growth was sustained in this sample with crystals being more intergrown in nature. Limited number of particles showed some wavy edges, sliced at the edges or broken as well. In general, it was evident that although the particle size increased by the increase in linker quantity, the particles became polydisperse, agglomerated with tumor like growth. Therefore, the economical reactant composition was concluded to be 1:1 Zr:BDC.

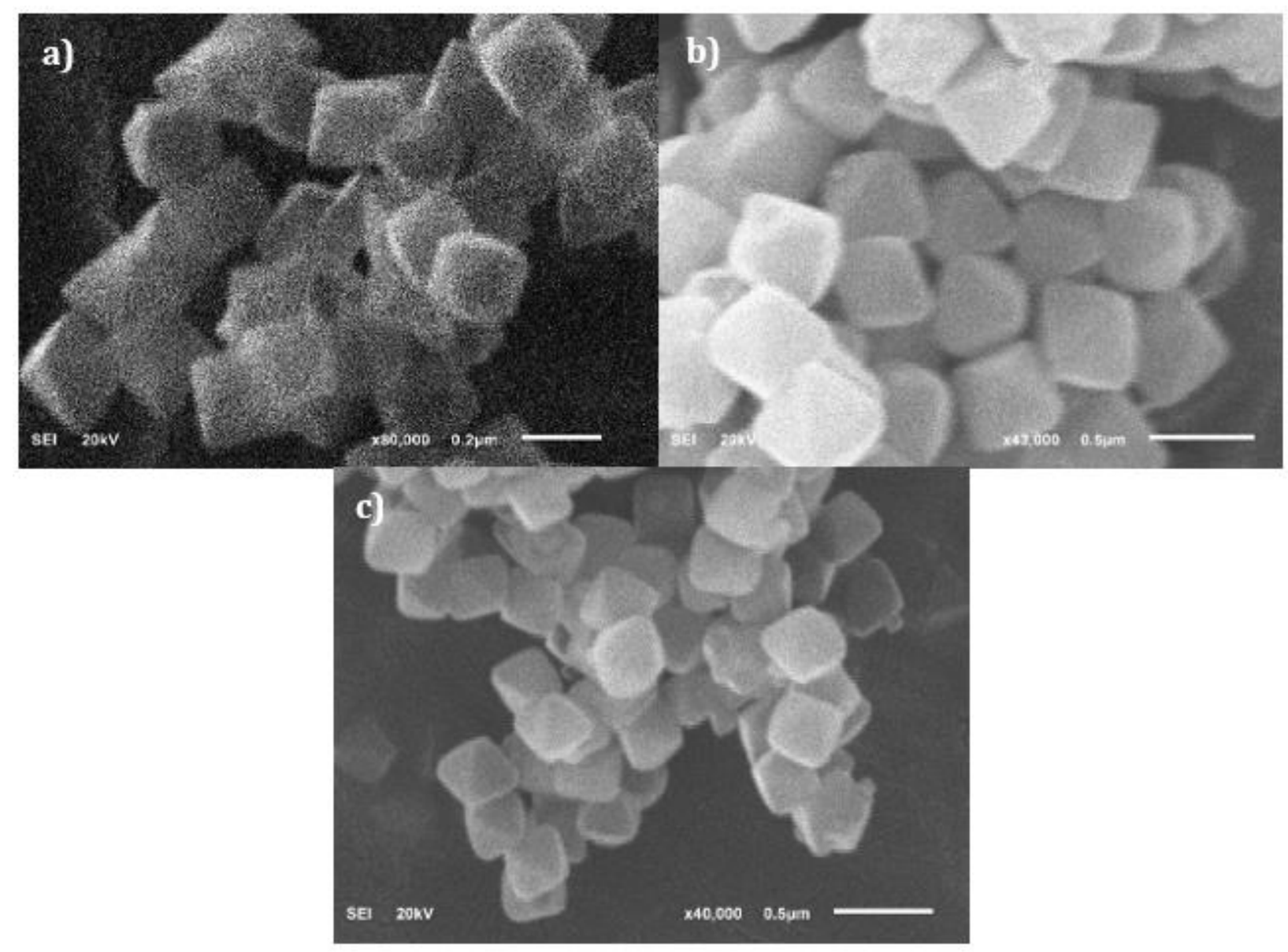

Figure 4.4.2-2: SEM images of UiO-66 with $\mathrm{Zr}$ (metal) as a limiting reactant: a) assynthesized $1: 1$, b) 1:1.5, and c) 1:2.

Following the same concept, the modification of morphology of UiO-66 was also studied by changing the $\mathrm{Zr}$ to ligand ratios as 1.5:1 and 2:1. The PXRD pattern, presented in Figure 4.4.2-3, it was evident that increasing the quantity of $\mathrm{Zr}$ to 1.5 and 2 times of BDC linker showed lower intensities in reference to the $2 \theta$ angles, 7.7 and $8.5^{\circ}$ but remained fairly similar in intensity for the rest of the diffraction pattern. The peaks also remained distinct and narrow with no merging of peaks for both samples. The peaks for 2:1 Zr:BDC ratio 
sample were much lower in intensity than $1.5: 1$, indicating reduction in crystallinity with increase in $\mathrm{Zr}$ ion content.

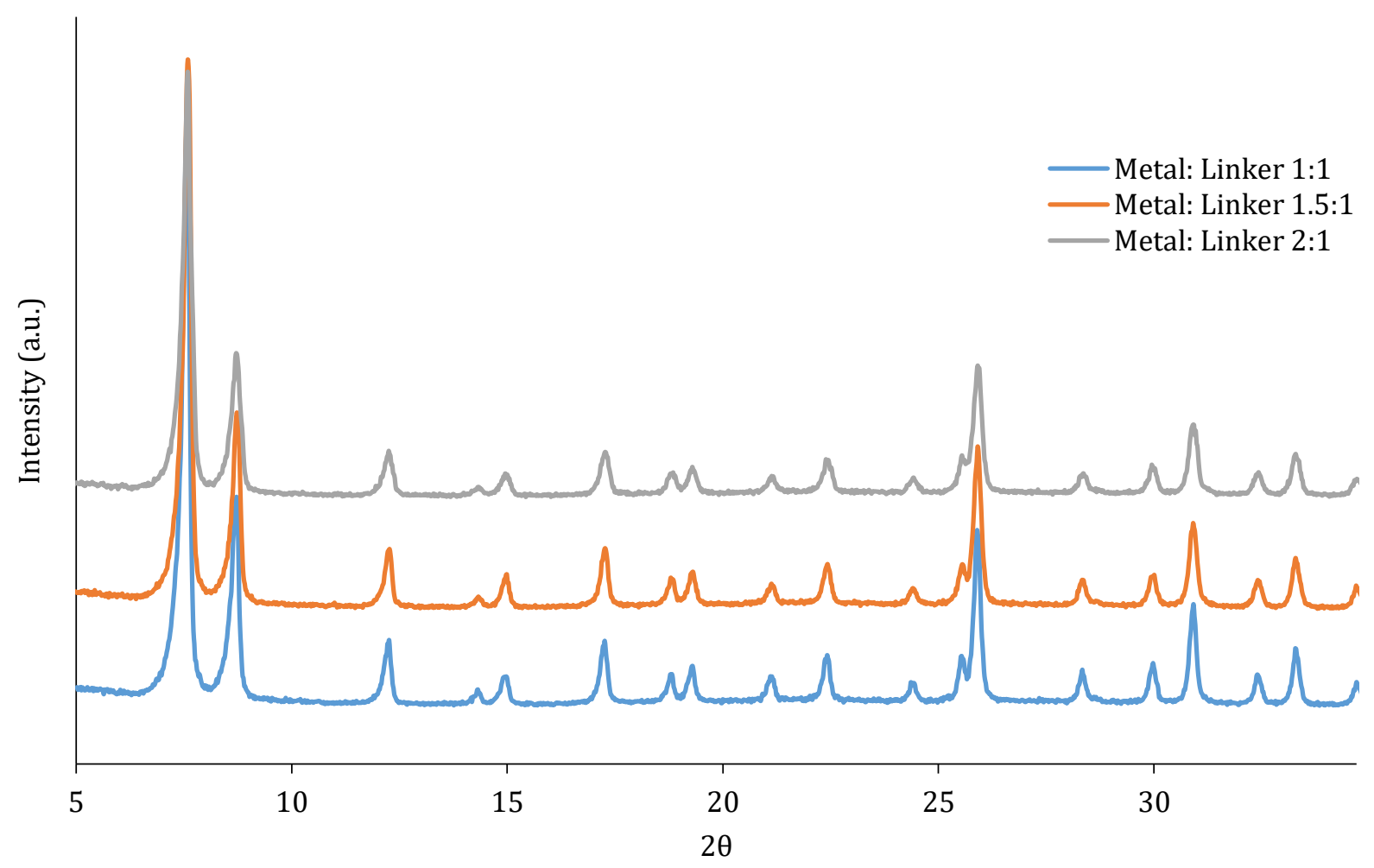

Figure 4.4.2-3: PXRD pattern of UiO-66 with BDC(ligand) as a limiting reactant.

The SEM of the samples, as pictured in Figure 4.4.2-4, revealed that both 1.5 increase in zirconium salt showed slightly blunt monodisperse octahedral crystals of $0.35 \mu \mathrm{m}$ with protuberances on the surface of the crystal in comparison to the 1:1 ratio that monodisperse sharp edged crystals of $0.2 \mu \mathrm{m}$ with no surface defects. Some crystals also appeared to be merged into one another. Further increase in zirconium up to 2 times produced a mixed sample of sharp and blunt edges octahedral with similar surface defects as with $1.5 \mathrm{Zr}$ sample. This sample appeared to be polydisperse with a size range of $0.2-0.4 \mu \mathrm{m}$. Hence, the appropriate ratio for producing monodisperse UiO-66 crystals is with a 1:1 ratio of metal: linker. 


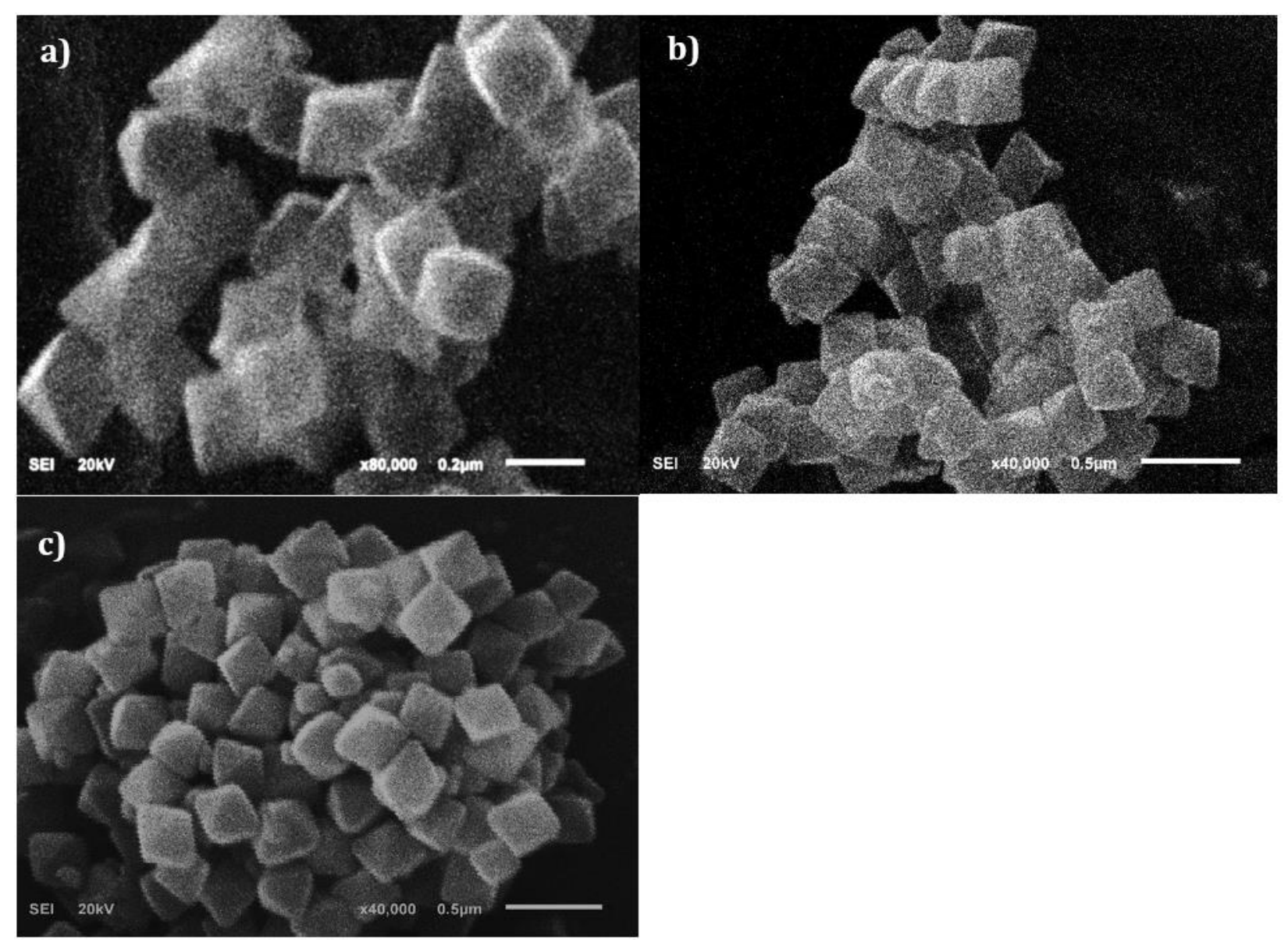

Figure 4.4.2-4: SEM images of UiO-66 with BDC(ligand) as a limiting reactant: a) assynthesized 1:1, b) 1.5:1, and c) 2:1.

The characterization results using the PXRD and SEM revealed that altering the ratio of 1:1 proved detrimental to the crystallinity of UiO-66. For the case of altering BDC amount, increasing the BDC content caused the crystallinity to drop. This observation is in line with the conclusions drawn by Zou et al. [98] where the PXRD pattern broadened upon decreasing the $\mathrm{Zr}: \mathrm{BDC}$ ratio. This was because the amount of BDC molecules remained unused in the reaction mixture. Additionally, Shearer et al. [99] determined that $\mathrm{Zr}: \mathrm{BDC}$ ratio exhibited minimal effect on the thermal stability.

Alternatively, the declination of crystallinity was also observed for increasing the quantity of metal salt which was a common observation from their study as well but was not conceptually accounted for. The best possible explanation for this trend could be that 
increasing the $\mathrm{Zr}$ ion concentration in the reaction increases the metal centers that form the primary nuclei. Since the nucleation stage is a competitive Ostwald ripening process, more nucleation centers compete to crystallize which slows down the growth of crystals. With the limited quantity of BDC available in the solution, poorly connected Zr clusters are formed, reflecting defective crystals. As a result, particle size increases upon increasing the zirconium concentration with respect to BDC. This information is also consistent with the increase in particle size of the samples with excessive $\mathrm{Zr}$ content, and thus, larger surface area. Consequently, in both instances, i.e., increasing the zirconium or terephthalic acid, crystallinity was reduced with appearance of surface defects in the form of protuberances.

\subsubsection{Conclusion}

This work attempted to evaluate the influence of altering the metal to ligand ratio on the seemingly perfect octahedral UiO-66 crystals by varying the $\mathrm{Zr}$ :BDC ratio as $1: 1.5,1: 2,1.5: 1$, and 2:1. Overall, there was no dramatic effect of changing the molar ratios of $\mathrm{Zr}$ and BDC on the characteristics of Uio-66. The addition of surplus $\mathrm{Zr}$ salt or BDC linker introduced polydispersity and/or surface defects in the resultant Ui0-66 crystals for the aforementioned reasons. Hence, the appropriate and economical composition for producing monodisperse UiO-66 crystals is with a 1:1 ratio of metal:linker.

\subsection{EFFECT OF REACTION TEMPERATURE}

\subsubsection{Introduction}

Many reports have considered temperature as being a strong operational variable in manipulating the morphology of the MOFs [100]. Its effects are vast ranging from the reaction kinetics to their dimensionality at the microscopic level [100]-[102]. Elevated temperatures, usually around an optimal, not only increase the reaction rate but also produce discrete and condensed structures that have high crystallinity while anything lower than the optimal temperature produces reduced crystallinity materials with low stability [43], [103]. Temperature also affects the solubility of the organic ligands, changes the 
adaptability of the organic ligands, changes the coordination modes of organic ligands and central metal atoms, and the energy barrier in high temperature syntheses [104].

In MOF frameworks, ligands also adapt different conformations with the change in reaction temperature. One such example was observed in the reaction of $\mathrm{Mn}$ (II) salt with $\mathrm{H}_{2} \mathrm{cpdba}$ and 2,2'-bpy that formed diverse MOF structures at different reaction temperatures [100], [105]. As seen in Figure $4.5 .1-1$, a $140^{\circ} \mathrm{C}$ reaction temperature formed a $2 \mathrm{D}$ network of $\left\{\left[\mathrm{Mn}(\mathrm{cpdba})\left(2,2^{\prime} \text {-bpy) }\right]\right\}_{\mathrm{n}}\right.$ (Figure $4.5 .1-1(\mathrm{a})$ ), while $150^{\circ} \mathrm{C}$ produced a complex 3D framework of $\left\{\left[\mathrm{Mn}_{2}(\mathrm{cpdba})_{2}\left(2,2^{\prime}-\mathrm{bpy}\right)\left(\mathrm{H}_{2} \mathrm{O}\right)_{2}\right] \cdot 3 \mathrm{H}_{2} \mathrm{O}\right\}_{\mathrm{n}}$ with unique building blocks of $\mathrm{Mn}_{3}$ (cpdba) $6\left(2,2^{\prime}\right.$-bpy) 2 (Figure 4.5.1-1(b)), and with an increase up to $170^{\circ} \mathrm{C}$, $\left\{\left[\mathrm{Mn}_{4}(\mathrm{cpdba})_{4}(2,2-\mathrm{bpy})_{3}\left(\mathrm{H}_{2} \mathrm{O}\right)_{2}\right]\right\}_{\mathrm{n}}$ (Figure 4.5.1-1(c)) was produced holding up the 3D structure with a decreased coordination number and water molecules. The dissimilarity in structures can be attributed to the adoption of different coordination and bridging modes of cpbda $^{2-}$, along with the cooperation of 2,2'-bpy ligand at different temperatures. Moreover, an additional coordination mode was adopted by the carboxylate group, transforming from Figure 4.5.1-1(b) to Figure 4.5.1-1(c), suggesting that an increase in coordination mode of central metal atoms and formation of M-O-M linkage which follows the temperaturecontrolled synthesis of MOFs.

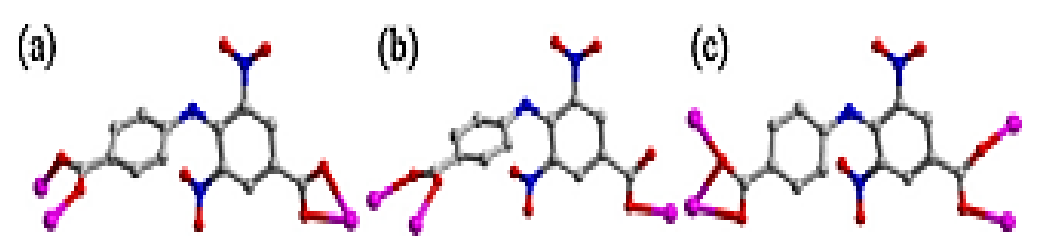

Figure 4.5.1-1: Coordination modes of $\mathrm{cpdba}^{2-}$ ligand with atoms indicated as pink $=\mathrm{Mn}$, red $=\mathrm{O}$, grey $=\mathrm{C}, \mathrm{H}$, and blue $=\mathrm{N}$ atoms where a) $140^{\circ} \mathrm{C}$ (bridging bidentate coordination), b) $150^{\circ} \mathrm{C}$ (no dominant bridging bidentate), and c) $170^{\circ} \mathrm{C}$ (monodentate) [100], [105].

Contrast to this case, the dimensions of several MOFs remain constant with only change in the orientation of the organic ligands. The example can be seen in the hydrothermal synthesis of $\mathrm{Co}\left(\mathrm{NO}_{3}\right)_{2} .6 \mathrm{H}_{2} \mathrm{O}$ with $\mathrm{H}_{2}$ bipa and titmb in aqueous solution that $\mathrm{Co}^{2+}$ ions formed square-pyramidal coordination geometry at $120^{\circ} \mathrm{C}$, while a reaction temperature of $180^{\circ} \mathrm{C}$ produced a complex distorted tetrahedral coordination geometry [102]. A stick diagram of 
the compounds (Figure 4.5.1-2a) at $120^{\circ} \mathrm{C}$ resembles a $2 \mathrm{D}$ honeycomb network structure with Co ion binding with three titmb ligands which are in turn connected to three Co ions. On the other hand, at $180^{\circ} \mathrm{C}$ (Figure 4.5.1-2b), a 3D network is formed by linking 1D Co-titmb chains to form [(Co) 6 (bipa) 6 ] metallocyclic rings [107]. In this case, the ligand titmb adopts unique conformations of cis-, trans-, trans-one in Figure 4.5.1-3(a) at $120^{\circ} \mathrm{C}$, and both cis-, trans-, trans- and cis-, cis-, cis-ones in (Figure 4.5.1-3(b) and Figure $4.5 .1-3(\mathrm{c})$ ) at $180^{\circ} \mathrm{C}$. This example elucidates that varying temperatures can change ligand conformations. Higher temperature encourages the molecules to form less favored thermodynamic configurations, which is otherwise not possible [100].

a)

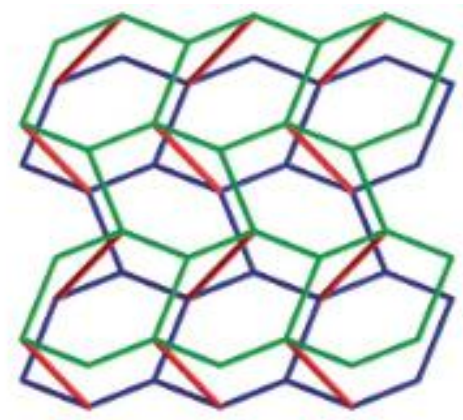

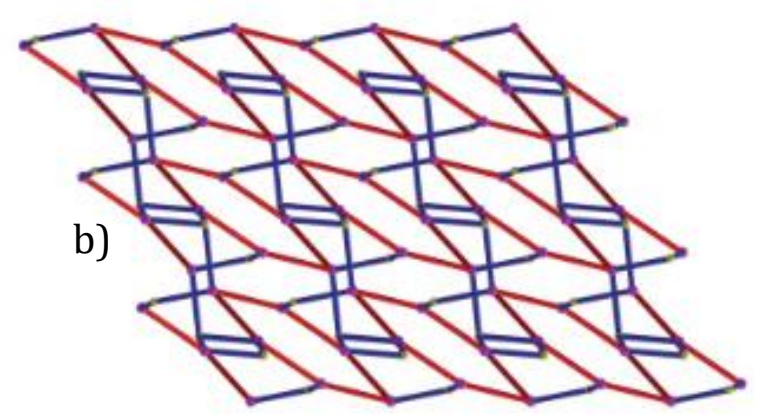

Figure 4.5.1-2: Schematic of $\mathrm{Co}(\mathrm{II}) \mathrm{MOF}$ at a) $120^{\circ} \mathrm{C}$ and b) $180^{\circ} \mathrm{C}$ [102].

(a)

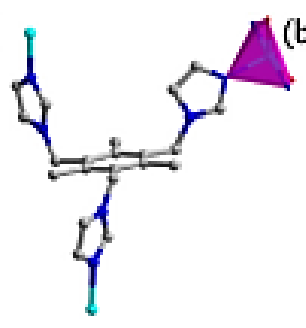

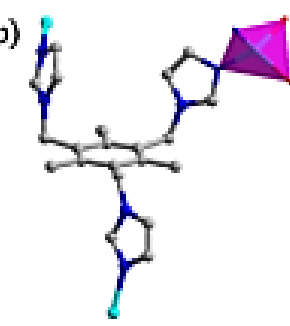

Figure 4.5.1-3: Conformation of titmb ligand and coordination geometry of Co(II) indicating N, C, 0 atoms in blue, grey, and red, respectively [100].

For UiO-66, a temperature of $120^{\circ} \mathrm{C}$ has been generally used for the solvothermal synthesis, with very little evaluation on using higher or lower temperatures. Hence, the temperature parameter was also studied as a part of the thorough systematic investigation of the effect of reaction variables on the morphology of UiO-66. The characterization results will help identify any phase and/or morphology changes and offer a better understanding of the 
development under different temperatures. Thus, in order to study the temperature effect on the morphology, UiO-66 was synthesized using Scheme 1 for $48 \mathrm{~h}$ at $100^{\circ} \mathrm{C}, 24 \mathrm{~h}$ at $120^{\circ} \mathrm{C}$, $16 \mathrm{~h}$ at $140^{\circ} \mathrm{C}$. As solvothermal synthesis of UiO-66 crystals is a thermodynamically driven process and temperature is directly associated with the supersaturation of the solution [106], the time period was chosen based on the assumption that an operational temperature of $100^{\circ} \mathrm{C}$ (lower than the published temperature of $120^{\circ} \mathrm{C}$ ) would take a longer time for the UiO-66 crystals to form. Also, the temperatures were chosen closer to the published temperature for relevance.

\subsubsection{Results and Discussion}

The goal of this experiment was to study the temperature effect on the morphology of UiO66 by subjecting the precursor solution to various synthesis temperatures, i.e. $100^{\circ} \mathrm{C}, 120^{\circ} \mathrm{C}$, and $140^{\circ} \mathrm{C}$ via Scheme 1 . The samples were stirred for $24 \mathrm{~h}$ and subjected to varying temperatures of $100^{\circ} \mathrm{C}$ at $48 \mathrm{~h}, 120^{\circ} \mathrm{C}$ at $24 \mathrm{~h}$, and $140^{\circ} \mathrm{C}$ at $16 \mathrm{~h}$, while keeping all other variables constant. The reactant concentrations and reaction conditions are summarized in Table 4.5.2-1. The as-synthesized sample was synthesized at $120^{\circ} \mathrm{C}$ at $24 \mathrm{~h}$ and is bolded for reference.

Table 4.5.2-1: Reactant composition and conditions to investigate the effect of reaction temperature.

\begin{tabular}{ccccccc}
\hline $\begin{array}{c}\text { ZrCl4 } \\
(\mathbf{m m o l})\end{array}$ & $\begin{array}{c}\mathbf{1 , 4 - B D C} \\
\text { (mmol) }\end{array}$ & $\begin{array}{c}\text { DMF } \\
\text { (eq.) }\end{array}$ & $\begin{array}{c}\text { Acetic } \\
\text { Acid } \\
\text { (eq.) }\end{array}$ & $\begin{array}{c}\text { Stirring } \\
\text { time (h) }\end{array}$ & $\begin{array}{c}\text { Temperature } \\
\left({ }^{\circ} \mathbf{C}\right)\end{array}$ & $\begin{array}{c}\text { Reaction } \\
\text { time (h) }\end{array}$ \\
\hline 0.343 & 0.343 & 993 & 30 & 24 & 140 & 16 \\
$\mathbf{0 . 3 4 3}$ & $\mathbf{0 . 3 4 3}$ & $\mathbf{9 9 3}$ & $\mathbf{3 0}$ & $\mathbf{2 4}$ & $\mathbf{1 2 0}$ & $\mathbf{2 4}$ \\
0.343 & 0.343 & 993 & 30 & 24 & 100 & 48 \\
\hline
\end{tabular}


A comparison of the diffraction patterns for all samples using an extended reaction time of 48h with the as-synthesized sample is presented in Figure 4.5.2-1. At first, the as-synthesized sample was compared to the sample produced at $120^{\circ} \mathrm{C}$ for $48 \mathrm{~h}$. The prolonged reaction time showed peak broadening along with significant intensity reduction. The twin peaks at $25.68^{\circ}$ merged to form a single peak, while the entire PXRD pattern at the higher angle appeared somewhat flat. This indicated reduction in crystallinity with extended reaction time of $48 \mathrm{~h}$. The $100^{\circ} \mathrm{C}$ PXRD pattern also revealed peak broadening and lower intensities and than the $120^{\circ} \mathrm{C}$ sample. The baseline of this pattern also showed a minor hump feature from $16.5^{\circ}$ to $27.5^{\circ}$ diffraction angle which may have appeared due to the presence of some amorphous material. Additionally, peak splitting was also identified in major peaks of this sample indicating formation of discrete particles formation. At $140^{\circ} \mathrm{C}$, the PXRD pattern turned out to be better in peak characteristics than the diffraction patterns at $100^{\circ} \mathrm{C}$ and $120^{\circ} \mathrm{C}$. The narrow and somewhat distinct peaks signalled better crystallinity and morphologies than other samples.

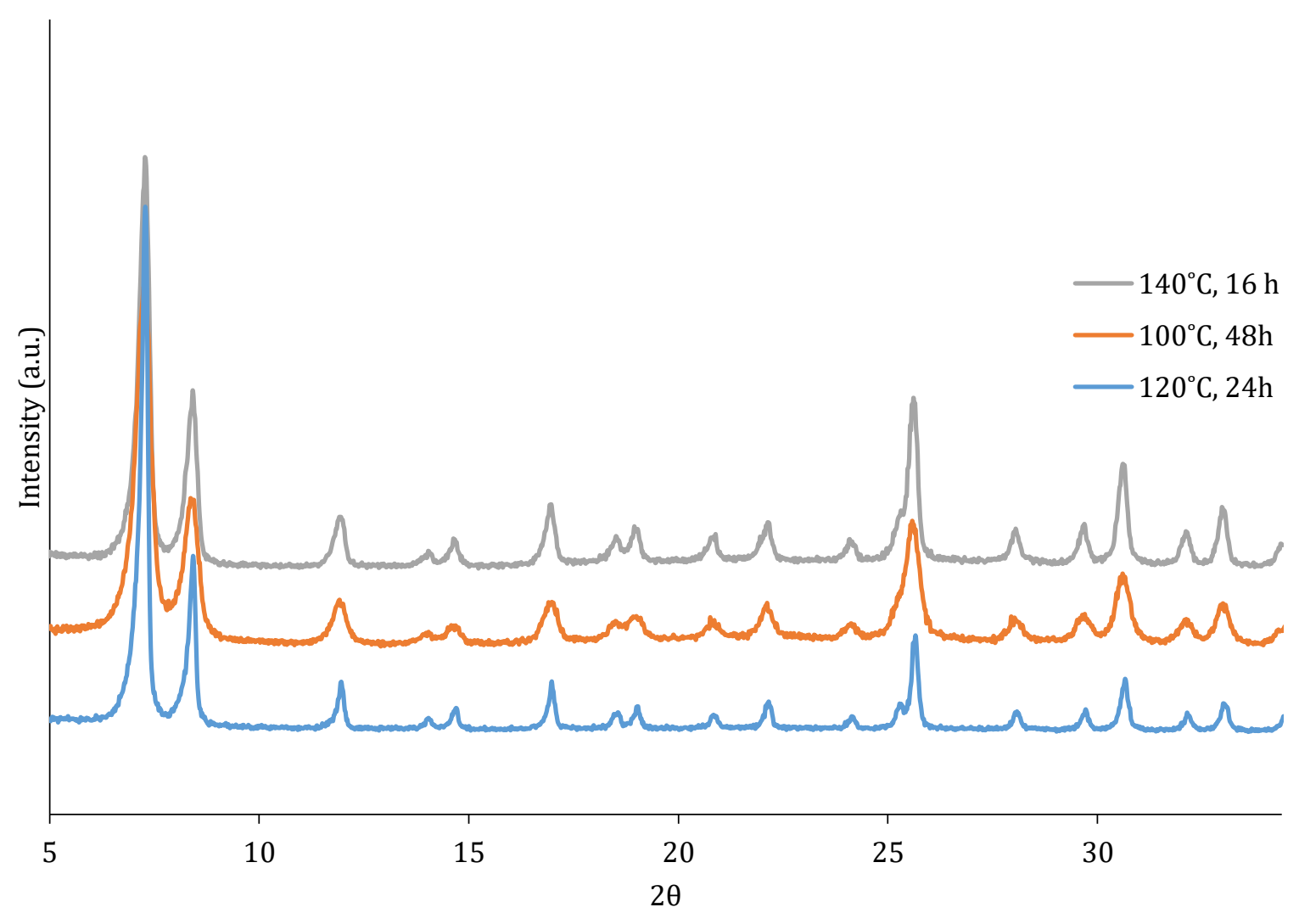

Figure 4.5.2-1: XRD patterns of UiO-66 for with varying temperatures. 
The SEM images of the UiO-66 samples for varying temperatures are depicted in Figure 4.5.22. At $100^{\circ} \mathrm{C}$, the particles appeared agglomerated with some areas of bigger ill-defined consolidated structures, which was also evidenced in the XRD pattern. Overall, these particles were polydisperse in nature. With increase in temperature, i.e. at $120^{\circ} \mathrm{C}$, the particle size was increased and discretized. Finally, at $140^{\circ} \mathrm{C}$, particles appeared to be discrete but polydisperse in nature.

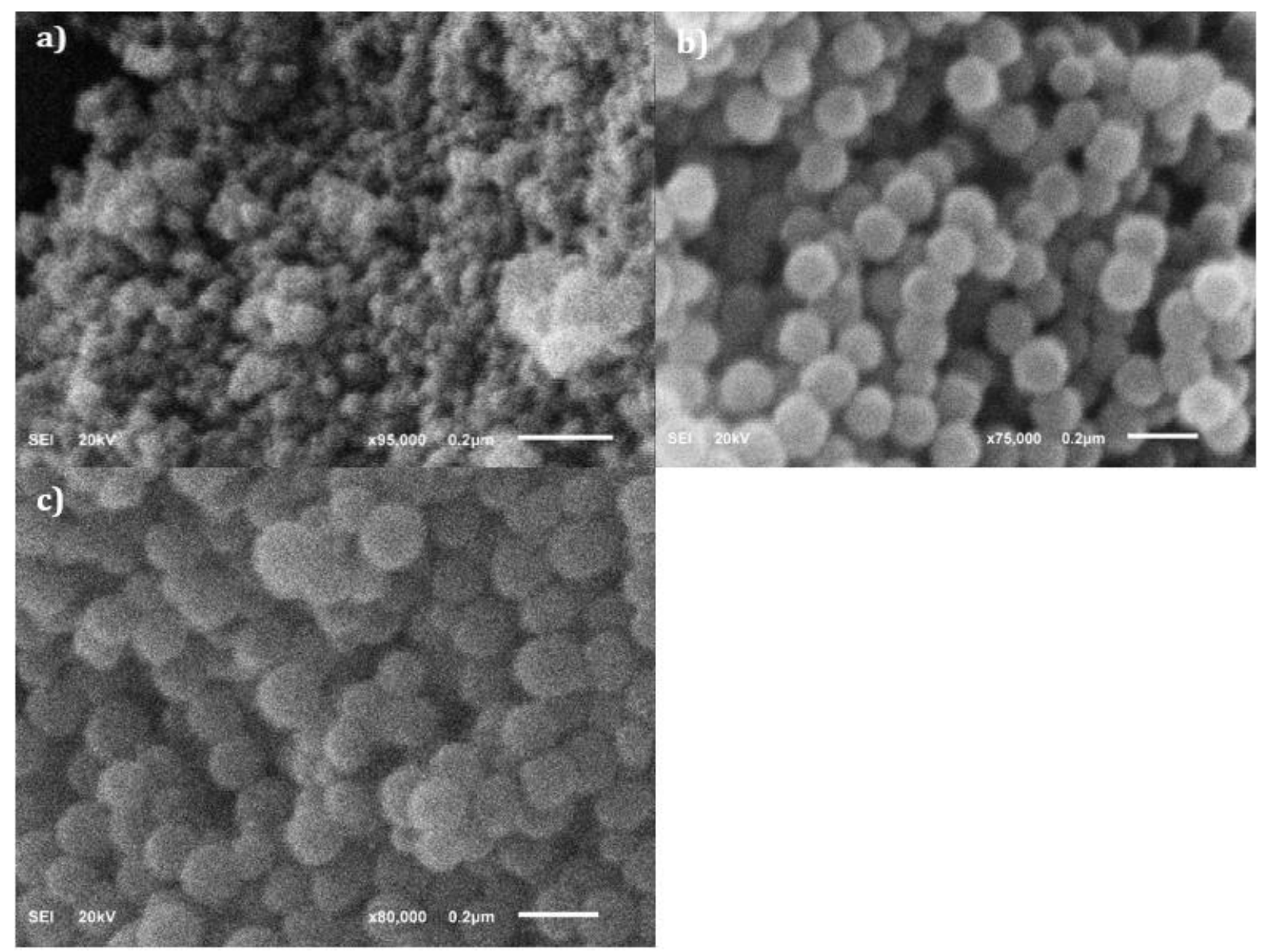

Figure 4.5.2-2: SEM images of UiO-66 produced at a) $100^{\circ} \mathrm{C}$ for $\left.48 \mathrm{~h} \mathrm{~b}\right) 120^{\circ} \mathrm{C}$ for $24 \mathrm{~h}$, c) $140^{\circ} \mathrm{C}$ for $16 \mathrm{~h}$.

A thorough analysis of the PXRD and SEM results proved that $120^{\circ} \mathrm{C}$ synthesis temperature was necessary for producing crystalline and discrete UiO-66 particles. It was also confirmed that all the synthesis temperatures can produce UiO-66 in pure phase with varying crystallinity. As supersaturation is also a function of synthesis temperature [106], a low synthesis temperature of $100^{\circ} \mathrm{C}$ produced slightly amorphous characteristics with partially ill-defined crystals and reduced symmetry. This may be attributed to the low kinetic energy 
of molecules at lower temperatures which made it difficult for the molecules to cross the thermal energy barrier and form stable nuclei and crystalline material [106]. Thus, the slow hydrolysis of $\mathrm{ZrCl}_{4}$ by carboxylate groups of the BDC linkers may have led to decreased crystallization and nucleation rates. In addition, the insufficient hydrolysis reaction may have led to deficit UiO-66 frameworks with vacant linker sites [99]. The peak splitting nature of the diffraction pattern can also be correlated to the irregular crystals in SEM image. Moreover, the number of defects could be high in the sample at room temperature as it is a prominent trait in samples synthesized at low temperatures [99]. Due to the sluggish crystallization rate at $100^{\circ} \mathrm{C}$, the particles may not have been fully formed and require longer reaction time. Thus, the slumped growth of particles and MOF formation would have resulted in small particle size and increased polydispersity.

Following the same concept, the state of the system at $140^{\circ} \mathrm{C}$ temperature raised the thermal energy of the system enabling more dissolutions of the solute particles, resulting in rapid growth of nuclei [43]. In this system, the solution achieved supersaturation faster than other synthesis temperatures, causing rapid nucleation and growth of crystals [106]. Hence, more particles were able to cross the activation energy to produce large UiO-66 crystals [107]. These crystals could not to be characterized with the given SEM resolution compared to the samples synthesized at lower temperature.

These observations were similar to the ones drawn by Shearer et al. [99] where the temperature effect was assessed for the UiO-66 samples at $100^{\circ} \mathrm{C}, 160^{\circ} \mathrm{C}$, and $220^{\circ} \mathrm{C}$, revealing that raising the temperature to $160^{\circ} \mathrm{C}$ yields better crystallinity than at $100^{\circ} \mathrm{C}$. Assessing the samples throughout the TGA showed the rate of loss of crystallinity was highest for sample subjected to $220^{\circ} \mathrm{C}$ which indicated that with increase in temperature, the sample became defective. The PXRD patterns revealed unusual reflections for miller indices (100) and (110) (at lower $2 \theta<7^{\circ}$ ), which was prominent for samples synthesized at low temperatures and frequently washed samples [99]. These reflections were attributed to defective sites within the systematic architecture. The defect regions or missing linker defects is referred as a BDC:cluster ratio of 4 which is lower than the ideal ratio of 6 in the as-synthesized UiO-66, suggesting missing linkers. This relationship was also in line with the BET surface area results: the highest BET surface areas corresponded to the highest 
linker deficient material. This indicated the increased number of defect sites with increase in the BDC concentration. Moreover, it was confirmed that the sites of linker deficiencies were terminated by chlorine among the possible terminal molecules such as formate, oxide, hydroxide or water.

Consistent observations were also made in the case of template based UiO-66 synthesis, wherein at lower synthesis temperature, the ligand remained unused in the reaction mixture yielding lower crystallinity, as opposed to higher temperature that lead to the development of impure crystals, due to the pyrolysis of ammonium salts [98].

In terms of phase purity, the samples formed at different temperatures maintained their phase purity with no intermediate phases. Within the given temperature range, this behavior is a contrast to other MOFs, where change in temperature normally causes change in ligand coordination numbers [101], [102]. In the formation of IRMOF-8 [108], different reaction temperatures revealed the formation of intermediate phases along with other products when the reaction temperature was different than the published synthesis temperature. Another study for the solvothermal formulation of HKUST-1 crystals revealed that the morphology changed from platelets to intergrown crystals with decreased regularity upon increasing the synthesis temperature [105]. The diffraction pattern also showed forbidden reflections at lower angles in the form of a hump in between the $4^{\circ}$ and $7^{\circ}$ that indicate the defective regions intergrown with regular framework [99]. Thus, it can be concluded that crystallinity drops past the best solvothermal synthesis temperature.

On a similar note, the results from the experiment at a temperature of $140^{\circ} \mathrm{C}$ led to the apparent hypothesis that a shorter reaction time may be required for producing UiO-66 at $140^{\circ} \mathrm{C}$. Thus, additional experiments were performed to investigate the appropriate reaction time at this temperature. To test this hypothesis, the experiments were conducted for reaction times of $16 \mathrm{~h}, 24 \mathrm{~h}, 48 \mathrm{~h}, 96 \mathrm{~h}$, at $140^{\circ} \mathrm{C}$ using Scheme 1. The characterization results confirmed that the highest crystallinity at $140^{\circ} \mathrm{C}$ among the given reaction times was achieved within $16 \mathrm{~h}$ of reaction time, which was consistent with the theory [106]. However, the crystallinity for this sample remained well below the as-synthesized sample. The best possible reason could be the increased number of defects that reduce the symmetry of the 
UiO-66 crystals. Therefore, an operational temperature of $140^{\circ} \mathrm{C}$ is not desirable for attainting high crystallinity MOFs.

\subsubsection{Conclusion}

This experiment was performed to determine the impact of temperature on morphology of UiO-66 by subjecting it to different synthesis temperatures of $100^{\circ} \mathrm{C}, 120^{\circ} \mathrm{C}$, and $140^{\circ} \mathrm{C}$ while keeping all other parameters constant. Based on the characterization results, it was proved that a lower temperature caused sluggish crystallization rates resulting in ill-defined Ui0-66 crystals, while a higher temperature accelerated the reaction rate leading to undesirable small UiO-66 crystals. Therefore, considering the overall outcomes of different synthesis temperatures to produce UiO-66, it is safe to conclude that the UiO-66 crystals are best produced according to Scheme 1 are at $120^{\circ} \mathrm{C}$ synthesis temperature for $24 \mathrm{~h}$ with $24 \mathrm{~h}$ stirring time.

\subsection{EFFECT OF MODULATOR CONCENTRATION}

\subsubsection{Introduction}

Modulators can be viewed as one of the most effective compositional variables in changing the geography of MOFs. MOFs were initially synthesized in solvent without a modulator. The crystals produced from this type of synthesis resulted in poorly formed polycrystalline powder with low crystallinity [4], [68]. This property was ascribed to the high charge density of the zirconium atoms. The strong electrophilic character polarized the $\mathrm{Zr}-\mathrm{O}$ bond reducing the ligand exchange between the metal and linker molecules [4]. Therefore, the unfavorable process resulted in fewer high-quality crystals [4]. Consequently, the modulated synthesis technique was adopted in order to produce discrete crystals [4].

To highlight a few remarkable findings where the modulator plays a crucial role in tuning the shape and size in the solvothermal synthesis of UiO-66 are presented here. One of the first coordination modulation methods proposed by A. Schaate et al. [99] proved the importance of the role of acetic acid, benzoic acid and water on the synthesis of UiO-66, UiO- 
66- $\mathrm{NH}_{2}$, UiO-67, and UiO-68- $\mathrm{NH}_{2}$. It was hypothesized that acetic acid affected the nucleation rate by slowing down the nucleation process and gradually decreasing the growth of the particles during the synthesis [99]. The addition of acetic acid thus transformed the crystals from being agglomerated to large single discrete crystals which was established by the obtained crystallite sizes of $72 \mathrm{~nm}$ with no acetic acid to $171 \mathrm{~nm}$ with 30 eq. acetic acid [99]. Furthermore, both SEM images and BET surface areas changed from $700 \mathrm{~m}^{2} / \mathrm{g}$ with no acetic acid to $1400 \mathrm{~m}^{2} / \mathrm{g}$ in the presence of 30 equivalent acetic acid [99]. Similar conclusions were drawn through other modulated syntheses that, increasing the amount of modulator not only changes the shape of the crystal but also, in general, increases the particle size [68], [109].

Another interesting aspect of using the modulator is controlling the number and nature of defect sites in the crystal lattice. The linker vacancies can be adjusted to vary the pore volume from 0.44 to $1.0 \mathrm{~cm}^{3} / \mathrm{g}$, with BET surface areas ranging from 1000 to $1600 \mathrm{~cm}^{3} / \mathrm{g}$, respectively, by change in modulator concentration [4], [110]. Therefore, ratio of the modulator to the metal source can be adjusted to produce a crystalline structure with required defect concentration, pore volume and surface areas.

In general, the quantity of acetic acid helps in regulating the nucleation rate. Increasing the concentration of acetic acid limits the amount of BDC linker connecting the zirconium atom, yielding large discrete crystals. As reported in literature, the current use of acetic acid as a modulator with a 30 eq. concentration to $\mathrm{Zr}$ produces well-defined sharp-edged octahedral crystals. The concentration of acetic acid was regulated in this synthesis recipe to observe the change in morphology and if it can further be improved. This theory was tested by applying Scheme 1 and varying the concentration of acetic acid as $0.0103 \mathrm{~mol}$, i.e. 30 eq. (assynthesized), $0.03 \mathrm{~mol}, 0.06 \mathrm{~mol}, 0.09 \mathrm{~mol}, 0.12 \mathrm{~mol}$. It was found that all concentrations of Ui0-66 successfully produced pure phase UiO-66, with crystallinity of particles only increasing up to a $0.03 \mathrm{~mol}$ of acetic acid. 


\subsubsection{Results and Discussion}

The main purpose of this experiment was to investigate the crystal growth observed on altering the concentration of acetic acid as a modulator and to determine the concentration of acetic acid that would produce the best crystallinity of UiO-66 with the adopted solvothermal synthesis recipe. The samples were stirred for $24 \mathrm{~h}$ and subjected to $120^{\circ} \mathrm{C}$ for $24 \mathrm{~h}$ by increasing the acetic acid concentration from $0.0103 \mathrm{~mol}$ to $0.12 \mathrm{~mol}$. The reactant concentrations and reaction conditions are summarized in Table 4.6.2-1. The as-synthesized sample was synthesized at $120^{\circ} \mathrm{C}$ with the published acetic acid concentration of $0.0103 \mathrm{~mol}$ and is bolded for reference.

Table 4.6.2-1: Reactant composition and reaction conditions to investigate the effect of modulator concentration.

\begin{tabular}{ccccccc}
\hline $\begin{array}{c}\text { ZrCl4 } \\
\text { (mmol) }\end{array}$ & $\begin{array}{c}\text { 1,4-BDC } \\
\text { (mmol) }\end{array}$ & $\begin{array}{c}\text { DMF } \\
\text { (eq.) }\end{array}$ & $\begin{array}{c}\text { Acetic } \\
\text { Acid } \\
\text { (mol) }\end{array}$ & $\begin{array}{c}\text { Stirring } \\
\text { time (h) }\end{array}$ & $\begin{array}{c}\text { Temperature } \\
\left({ }^{\circ} \mathbf{C}\right)\end{array}$ & $\begin{array}{c}\text { Reaction } \\
\text { time (h) }\end{array}$ \\
\hline $\mathbf{0 . 3 4 3}$ & $\mathbf{0 . 3 4 3}$ & $\mathbf{9 9 3}$ & $\mathbf{0 . 0 1 0 3}$ & $\mathbf{2 4}$ & $\mathbf{1 2 0}$ & $\mathbf{2 4}$ \\
0.343 & 0.343 & 993 & 0.03 & 24 & 120 & 24 \\
0.343 & 0.343 & 993 & 0.06 & 24 & 120 & 24 \\
0.343 & 0.343 & 993 & 0.09 & 24 & 120 & 24 \\
0.343 & 0.343 & 993 & 0.12 & 24 & 120 & 24 \\
\hline
\end{tabular}

The diffraction patterns for the samples are presented in Figure 4.6.2-1. UiO-66 was effectively produced for all the acetic acid concentrations. Comparing the PXRD peaks to the reference of the as-synthesized sample at $2 \theta$ of $7.48^{\circ}$, the highest peak intensity was observed for $0.03 \mathrm{~mol}$ followed by $0.06,0.09,0.0103$, and $0.12 \mathrm{~mol}$. As per this observation, a clear trend was identified that, on increasing the molar concentration of acetic acid up to 0.03mol, the crystallinity was higher compared to $0.0103 \mathrm{~mol}$ acetic acid (as-synthesized), indicating a larger particle size. This was clearly reflected from the diffraction peak as the 
peaks narrowed and intensified for this sample. Increasing the acetic acid concentration to $0.06 \mathrm{~mol}$ caused peak broadening and lower peak intensities. When the concentration was increased further to a $0.09 \mathrm{~mol}$ of acetic acid, the crystallinity dropped further which was signalled by the occurrence of ill-defined peaks at higher angle with appearance of double peaks at $28.5^{\circ}$. However, the peak intensities for the diffraction pattern of 0.06 and $0.09 \mathrm{~mol}$ of acetic acid were comparable. Finally, when the acetic acid concentration was increased to $0.12 \mathrm{~mol}$, the peaks appeared to be broadened, followed by drastic drop in intensity. The pattern for $0.12 \mathrm{~mol}$ showed a hump between $12.8^{\circ}$ and $29.5^{\circ}$ with distorted peaks and lowest intensity over the entire range. The peak at $7.48^{\circ}$ for this sample also showed peak splitting which indicated reduced symmetry or a different possible phase. Thus, it was evident that increasing the amount of acetic acid past the $0.03 \mathrm{~mol}$ concentration reduced the crystal size and crystallinity.

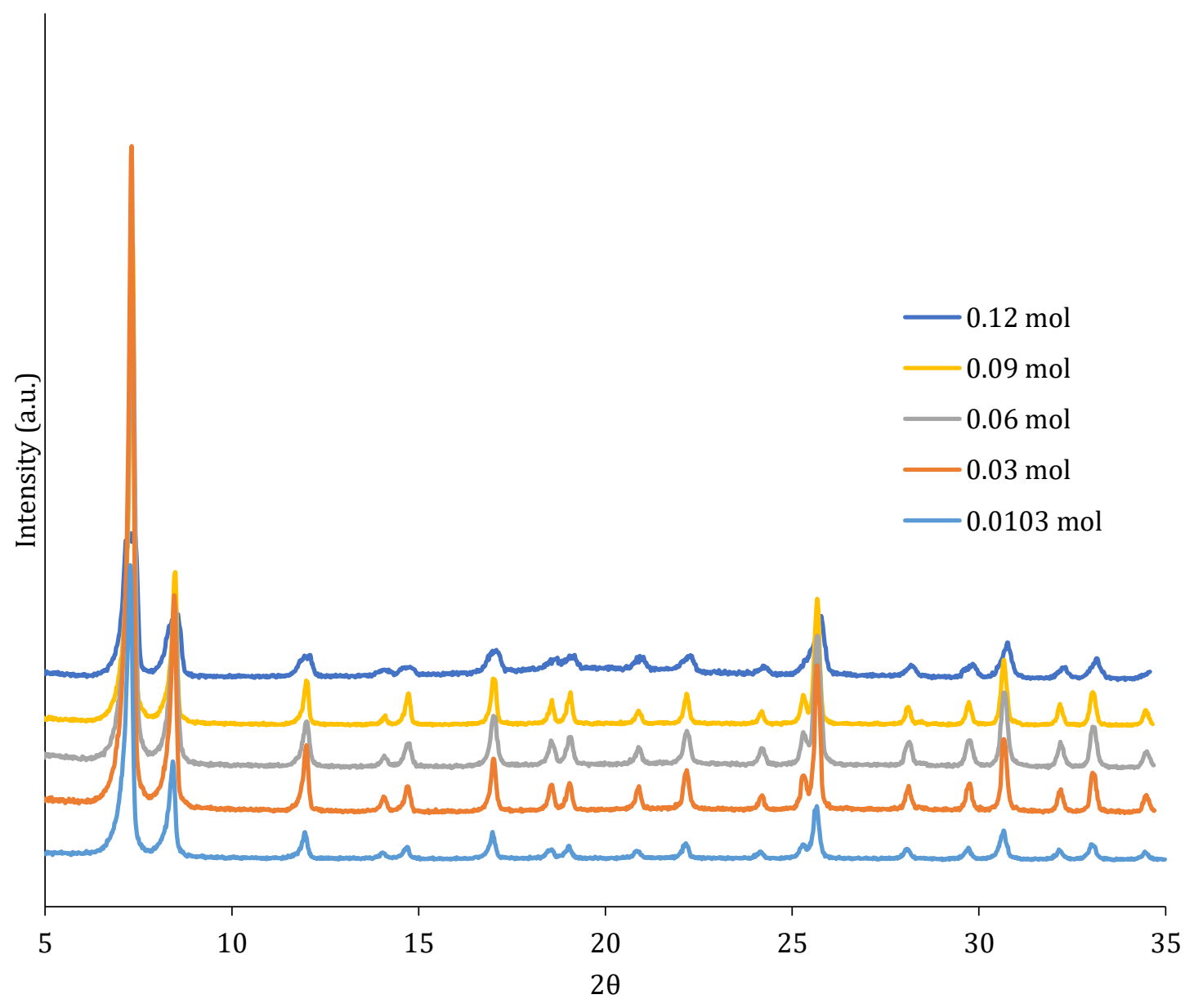

Figure 4.6.2-1: PXRD pattern of UiO-66 for different acetic acid concentrations. 
The SEM images of the samples, as shown in Figure 4.6.2-2, showed sharp edged, highly crystalline morphologies upon increasing the quantity of acetic acid. In comparison to the samples with higher acetic acid content, the as-synthesized particles appeared small, intergrown crystals with about $0.12 \pm 0.015 \mu \mathrm{m}$ in size and uniform in nature. The shape of these particles was unclear due to the limited SEM resolution but appeared faceted with rounded edges. Upon increasing the concentration to $0.03 \mathrm{~mol}$, the particles grew larger with rounded square bipyramidal structures of about $0.19 \pm 0.02 \mu \mathrm{m}$ in size. These particles, however, were polydisperse and intergrown in character with surface defects. Further increase in acetic acid to 0.06 and $0.09 \mathrm{~mol}$ produced sharp edged octahedral polydisperse crystals with largest crystal sizes being $0.26 \pm 0.048 \mu \mathrm{m}$ and $0.34 \pm 0.170 \mu \mathrm{m}$, respectively. These samples were also observed to have increased in polydispersity with the given acetic acid content. With $0.09 \mathrm{~mol}$ acetic acid, some crystals also appeared to be conjoined. Lastly, increasing the acetic acid concentration to 0.12 mol produced defective intergrown octahedral crystals of about $0.53 \pm 0.059 \mu \mathrm{m}$. The crystals also appeared to be truncated at some edges while being conjoined. 

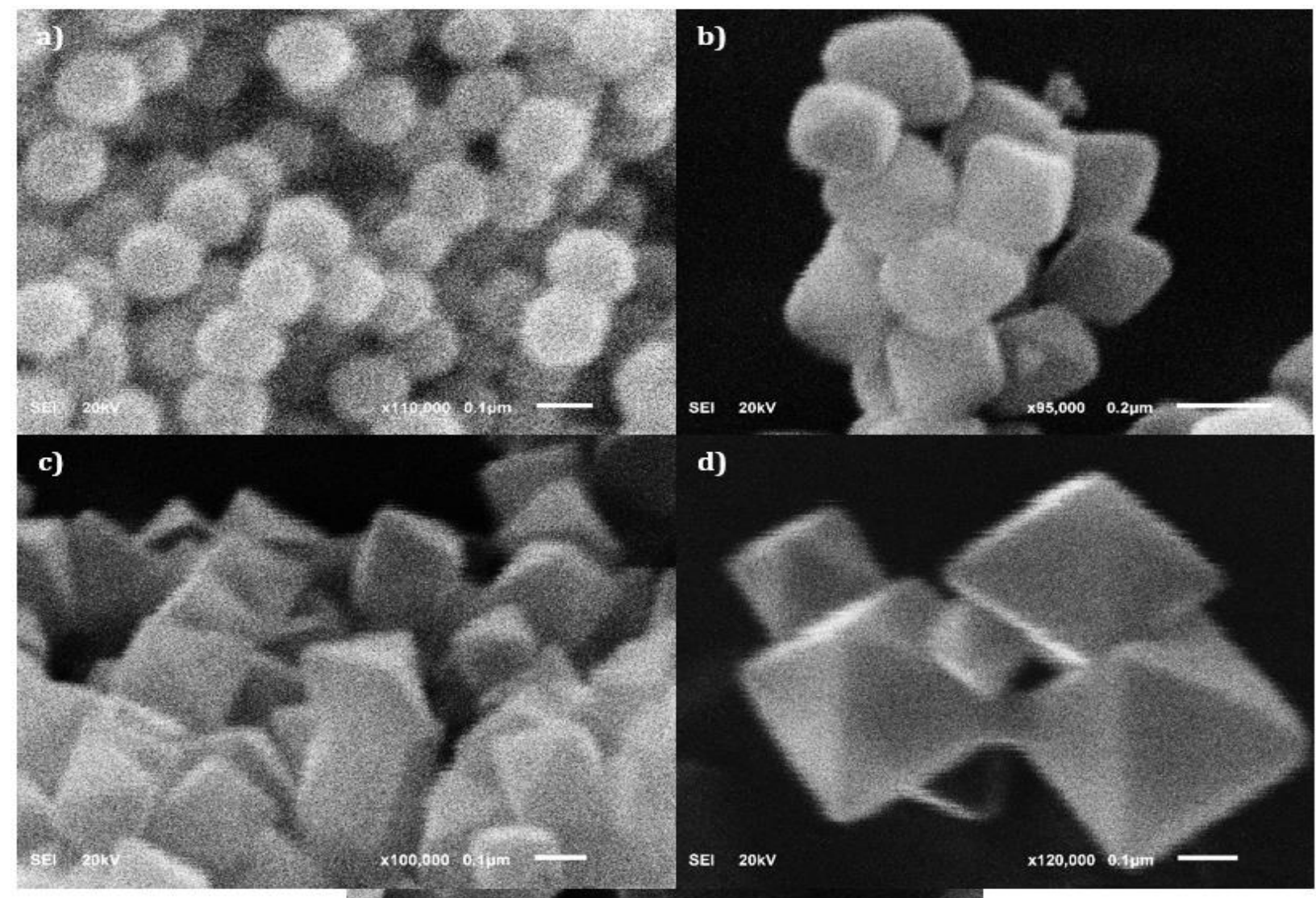

d)

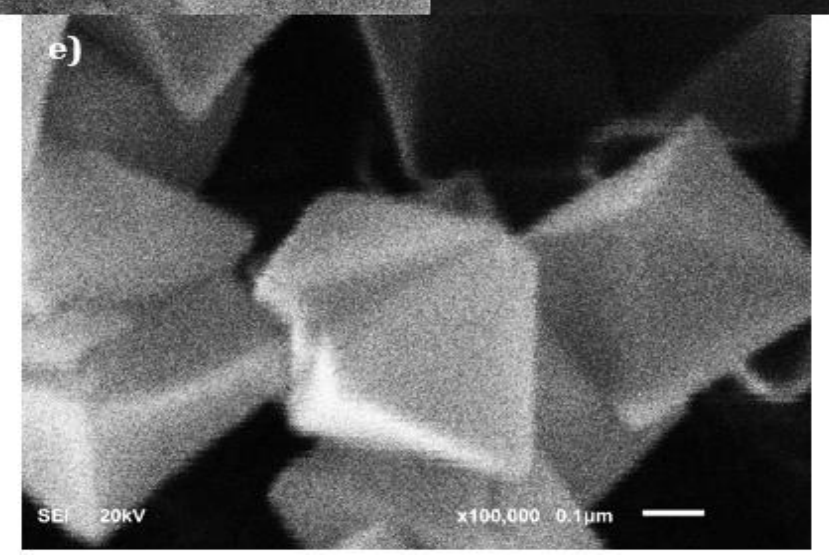

Figure 4.6.2-2: SEM images of UiO-66 at acetic acid concentrations at a) $0.0103 \mathrm{~mol}, \mathrm{~b}$ ) $0.03 \mathrm{~mol}, \mathrm{c}) 0.06 \mathrm{~mol}$, d) $0.09 \mathrm{~mol}$, and e) $0.12 \mathrm{~mol}$. 


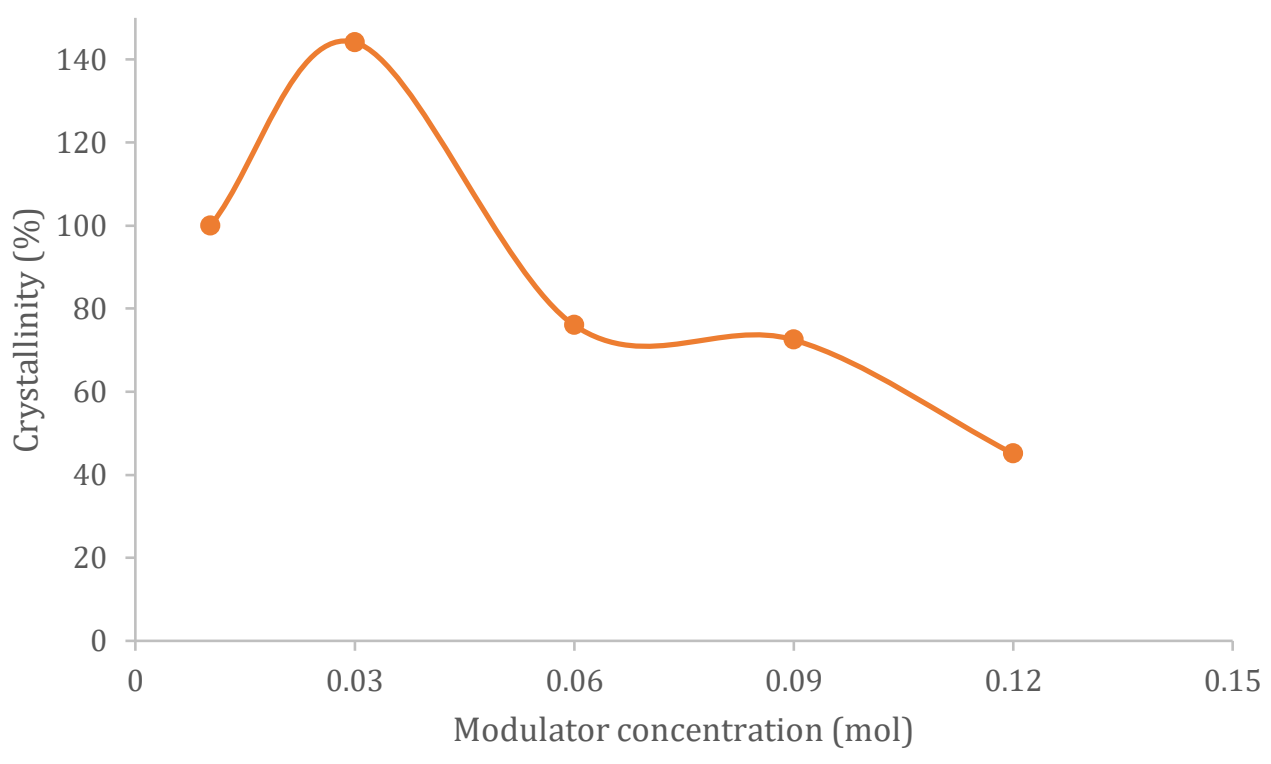

Figure 4.6.2-3: Effect of modulator concentration on the crystallinity of UiO-66.

The crystallinities were calculated by the peak ratio method introduced in Section 4.2.2. The effect of modulator concentration on the crystallinity of UiO-66 particles is summarized in Figure 4.6.2-3. As observed, the diffraction experiments showed that increasing the quantity of acetic acid to $0.03 \mathrm{~mol}$ increased the crystallinity of the UiO-66 crystals but produced surface defects, as observed from SEM images. Compared to the as-synthesized sample, increasing the concentration of acetic acid to $0.03 \mathrm{~mol}$ increased the particle size of UiO-66 but indicated non-uniformity. The non-uniformity of the particles increased with the surplus amount of acetic acid was very well reflected from the PXRD and SEM. The major difference in the diffraction pattern was observed for the peaks at $7.3^{\circ}$ and $8.5^{\circ}$ while the rest of the pattern did not indicate significant change. The reduction in intensities with these two specific peaks indicated the particle growth was suppressed in that direction. In addition, the drop and broadening of peaks indicated reduced structural order.

From literature, the effect of modulator on the formulation of UiO-66 is that it thermodynamically regulates the equilibrium by impeding the interaction between metal and linker molecules. The modulator affects the morphology in the following ways; firstly, it acts as a capping agent that prevents the layered growth and crystal aggregation, but leaves the pillaring direction unaltered [27], [111]. It also efficiently competes with the linker for the coordination site of the central metal cation but should not completely displace the linker 
[26], [112]. In case of UiO-66, a modulator promotes the formation of in-situ coordination complexes between zirconium and monotopic carboxylic acid linkers [4]. The modulators compete with the linker molecules and bind with the zirconium atoms or similar complex to the carboxylic acid molecules [4]. These new SBUs formed through an exchange reaction between ligands and themselves become the transitional complexes in building the product [4]. If chosen correctly, an increase in the modulator concentration impedes the binding of linkers to the metal center [4]. This in turn limits the growth and number of nucleation sites, thereby, enhancing the growth of large discrete crystals [109].

A thorough study by Shearer et al. [99] exploring the defect chemistry of Ui0-66 identified two important characteristics of the addition of monocarboxylic acid modulators in the solution that: (1) increase in modulator content systematically increased the number of linker deficiencies in the framework except for acetic acid, where the linker deficiency density was indifferent unless high contents of modulator $\sim 100$ eq. were added to the solution. (2) The linker deficiency density increased with the increase acidity/p $K_{a}$ of the modulator [99]. The increase in number of linker deficiency were correlated to increased porosity and surface area of UiO-66 crystals. The characterization of the crystals revealed that the occurrence was due to the higher $\mathrm{p} K_{a}$ values of modulator acids than the BDC linkers. The higher $\mathrm{p} K_{a}$ value ensured more deprotonation of modulator ligands than BDC ligands within the solution that showed higher affinity to $\mathrm{Zr}$ atoms [113]. It was confirmed that the charge for the BDC molecules were compensated by the acetate ions from the modulator as well as the formate ions (originating from the in-situ decomposition of DMF during the MOF synthesis). As a result, the connectivity of $\mathrm{Zr}$ atoms changed from 12 of $\mathrm{Zr}$ to a coordination number of 8 with larger number of defects due to the increased quantity of modulator [114]. Thus, the mechanism was summarised in the following order: high concentration of modulator - more deprotonated modulator in mixture - increased binding of deprotonated modulator to clusters - higher concentration of defects.

These observations are equivalent to the ones observed by Lu's group [68] who reported change in the crystal size on varying the acetic acid concentration. The absence of acetic acid resulted in the production of agglomerated crystals, while the increase in concentration of acetic acid produced monodisperse discrete octahedral crystals with an average crystal size 
of $409 \pm 29 \mathrm{~nm}$, indicating the effect of acetic acid on the nucleation. The surface area also increased from $773 \mathrm{~m}^{2} / \mathrm{g}$ in the absence of acetic acid to $1376 \mathrm{~m}^{2} / \mathrm{g}$. This trait was attributed to the fact that increase in acetic acid increased the amount of deprotonated acetate molecules which competed with the deprotonated linker molecules [114]. As a result, the acetate molecules occupied many locations of the BDC linker molecules and became part of the UiO-66 framework creating locations of missing linker and cluster defects within the molecule [114]. In addition, the intended regulation slowed down the nucleation process leading to large UiO-66 crystals with large surface areas [114]. Hence, the modulator affected both the crystal size of the MOF.

Another pioneering report in the modulated synthesis was demonstrated by Kitagawa group in 2010 with the synthesis of a HKUST-1 [109]. Non-modulated synthesis of [ $\mathrm{Cu}_{3}(1,3,5-$ btc) 2$]_{\mathrm{n}}$ in butanol produced gel-like precipitate with poor crystallinity; while the addition of dodecanoic acid yielded high quality cubic shaped crystals with size increasing from $20 \mathrm{~nm}$ to $1 \mathrm{~mm}$ upon increasing the modulator to ligand ratio. Similarly, the synthesis of HKUST-1 from copper nitrate produced octahedron, cuboctahedron and cubic crystals on altering the modulator concentration [109]. The competitive nature of the modulator prevents the over saturation of the solution with precursors thereby slowing down the nucleation rate. As a result, the limited number of nuclei grow into larger crystallites upon increasing the modulator concentration [109]. Therefore, altering modulator concentration offer tuning of crystal surfaces and virtually regulate surface reactivity.

\subsubsection{Conclusion}

In this specific experiment, the concentration of acetic acid was modulated in an effort to enhance the crystallinity of UiO-66 in a solvothermal synthesis process. Although the increase in particle size with the increase in concentration of modulator is true for this case, the crystallinity only rises to a concentration of $0.03 \mathrm{~mol}$ of acetic acid. Increasing the acetic acid content beyond $0.03 \mathrm{~mol}$ increased the particle size but decreased the crystallinity and homogeneity of the product. In addition, its increasing content proved to induce surface damages to the Ui0-66 crystals [43]. However, even a concentration of 0.03 mol introduced 
surface defects to the crystal which indicates increase in disorder. The increased particle size jeopardizes the crystallinity of the material leading to agglomerations and a large degree of non-uniformity in the sample. Contrastingly, if surface defects become desirable for an application such as catalysis, the higher concentration of acetic acid can be chosen likewise. Hence, it can be concluded that the appropriate concentration of acetic acid is at $0.0103 \mathrm{~mol}$ via Scheme 1. Consequently, this concentration of acetic acid was adopted for the impending syntheses.

\subsection{EFFECT OF CO-SOLVENTS}

\subsubsection{Introduction}

The change in the morphology of MOFs using solvents is quite ambiguous as solvents play several roles within the precursor solution [115]. Primarily, they set up the coordination atmosphere for the reactants while also serving as a medium of growth and interaction between metal centres and ligands or through other interactions within the framework [104]. Their dissociating components become a structure directing tool for the incoming organic molecules and indirectly control the formation of products through influencing the growth rate because of supersaturation and temperature [73], [104]. Also, they are often

identified as a part of the framework to counter balance the charges and/or occupy the interstitial spaces in the framework as guest molecules [104].

The most commonly used solvents in MOF synthesis are DMF, DEF and DMA. Some studies suggest that solvents may substitute themselves as ligands such as in the study of $\mathrm{Zn}$ benzobistriazolates, where, different composition of amide solvents in DMF vs DMA formed anatomically different products [43]. The MOFs produced using DMF show high porosity as opposed to the ones synthesized in DMA [43]. The MOFs produced in DMA contained DMA associated to central metal ions typically prove to be detrimental to its thermal stability. Thus, DMF is commonly employed as a solvent in the solvothermal synthesis of MOFs. However, both these synthesis routes evolved into architecturally different morphologies. 
There are numerous examples in the literature that demonstrate the dictated characteristic of solvent and co-solvents on the morphology of MOFs. Sindoro et al. [73] have exemplified this characteristic by producing shape-selectable MIL-96(Al) by varying the co-solvent ratios along with different solvents. As shown in Figure 4.7.1-1, MIL-96 using DMF:water ratio of 1:4 formed truncated hexagonal bipyramidal crystals while DMF:water ratio of 1:1 resulted in rounded bipyramidal crystals. Using a toluene-water (1:4) mixture and a tetrahydrofuran (THF)-water (1:4) mixture as the co-solvent, respectively, produced a hexagonal bipyramidal and spindle-shaped morphologies, respectively.

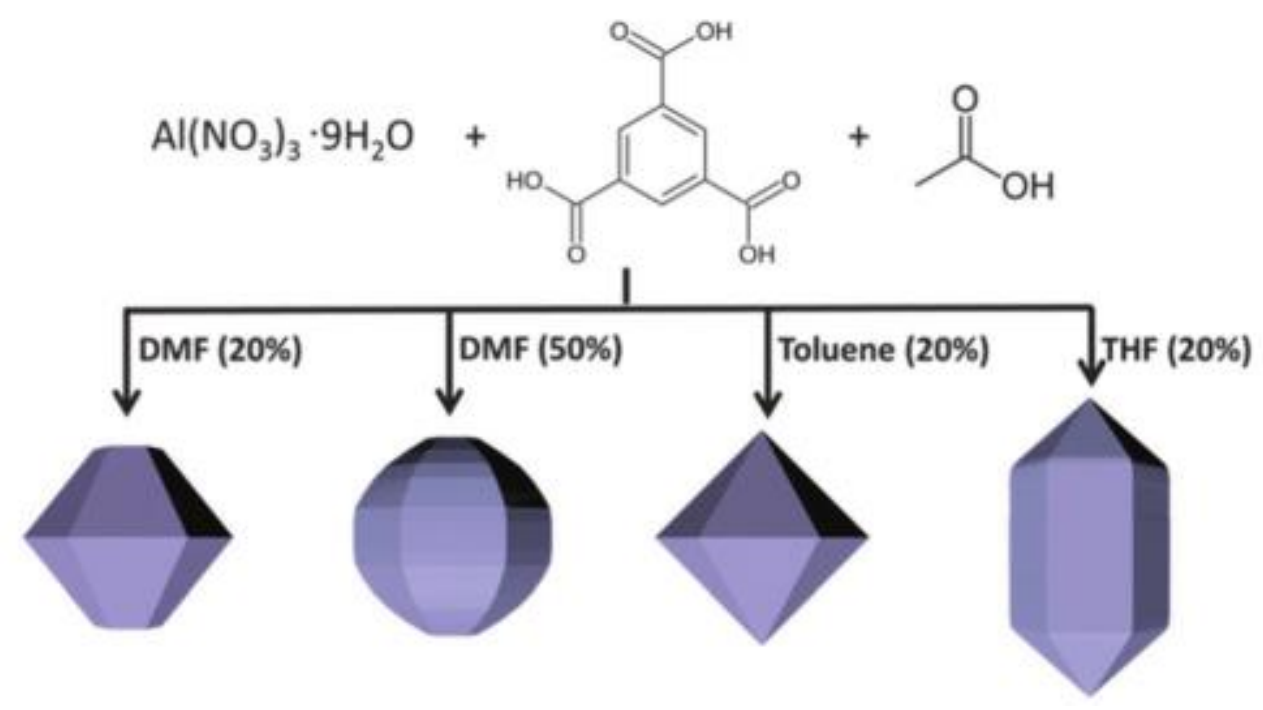

Figure 4.7.1-1: Different morphologies of MIL-96(Al) with various cosolvents [73].

Many other studies validate the judicious use of solvent concentration that ascribe structural transformations of MOFs as a direct consequence of the different interactions between solvent and metal ions that induces diverse coordination modes between linker molecules [104], [116]. A study led by Forster's group also reported different Mg-based MOFs by using $\mathrm{Mg}\left(\mathrm{NO}_{3}\right)_{2} \cdot 6 \mathrm{H}_{2} \mathrm{O}$ and 3,5-pdc under same synthesis conditions with different co-solvents, i.e. DMF, DMF+methanol, DMF+ethanol, ethanol+water [116]. As shown in Figure 4.7.1-2, all the obtained products were observed to be structurally different with diverse coordination modes between magnesium and pyridine dicarboxylate linker. The product synthesized from DMF based solvent contained isolated magnesium cornerstones of octahedral 3D 
framework with free DMF molecules occupying the network [116]. The DMF+methanol solvent yielded similar structure except with disoriented DMF and water molecules within the pores [116]. The DMF+ethanol also produced a similar 3D structure except without any free molecules lodging the channels; whereas, the ethanol+water solvent mixture produced an isolated 7-coordinated polyhedral structure, with water showing the highest affinity to magnesium ions compared to other solvents [116]. In addition, these transformations were ascribed to the interaction between the solvent and metal ions [116].

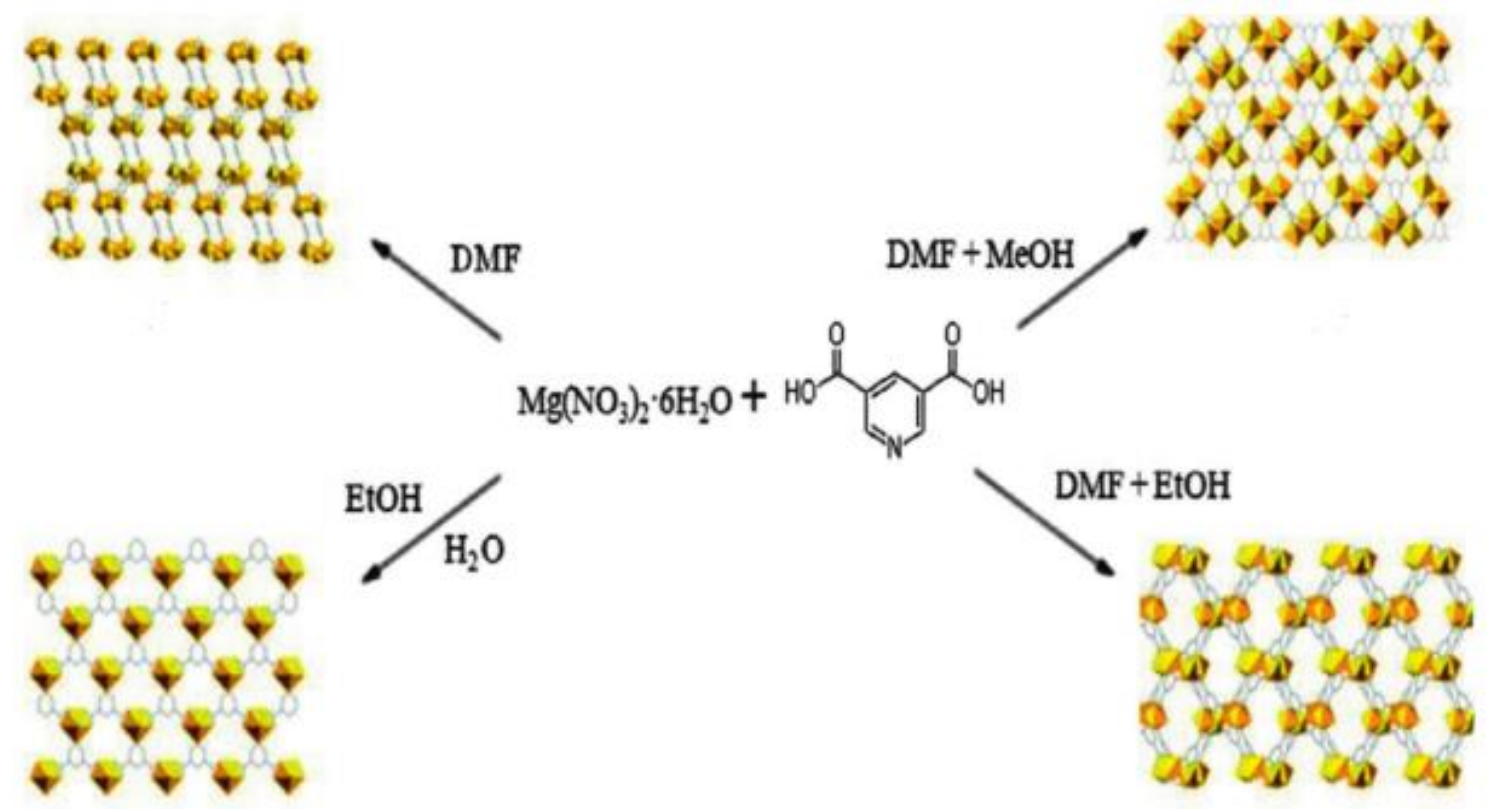

Figure 4.7.1-2: The self-assembly of $\mathrm{Mg}\left(\mathrm{NO}_{3}\right)_{2} \cdot 6 \mathrm{H}_{2} \mathrm{O}$ and 3,5-PDC under different solvents [104].

Another significant example of solvent effect comes from Luo's group that highlighted the structural diversities of Cd-based MOF from $\mathrm{Cd}\left(\mathrm{NO}_{3}\right)_{2} \cdot 4 \mathrm{H}_{2} \mathrm{O}$ and $\mathrm{H}_{3}$ bpt [117]. From Figure 4.7.1-3, a 3-dimensional network of $\mathrm{Cd}_{1}-\mathrm{O}-\mathrm{Cd}_{2}$ was formed with DMF bridges when using DMF as the solvent; while using DMA as the solvent produced infinite metal and carboxylic acid linkages with DMA connected to one of the cadmium ions [117]. However, the DEF solvent mixture contained 2-dimensional honey comb layer structure with mononuclear metal ions stacked upon each other [117]. 


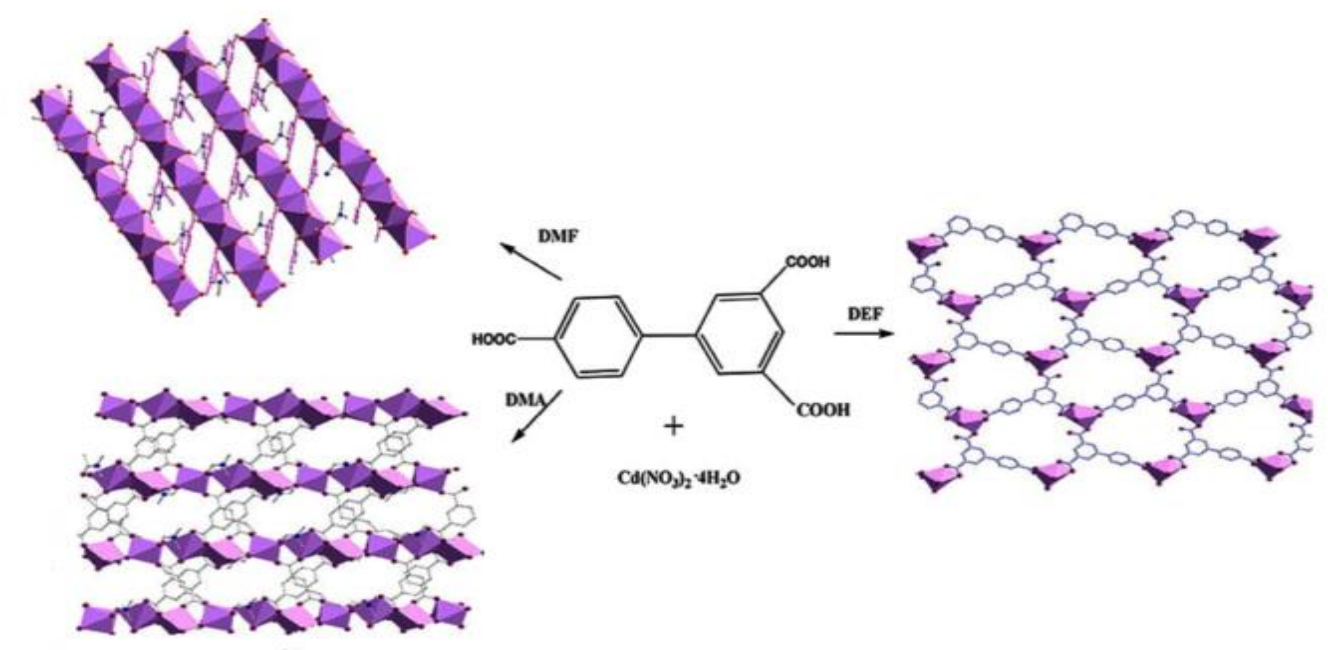

Figure 4.7.1-3: Coordination of $\mathrm{Cd}\left(\mathrm{NO}_{3}\right)_{2} \cdot 4 \mathrm{H}_{2} \mathrm{O}$ and $\mathrm{H}_{3}$ bpt by using different solvents [104].

The shape of Zr-MOF UiO-66 was initially reported as cubic intergrown crystals of about 200nm [37], which later evolved to octahedral bipyramidal shape through modulated synthesis [68]; while the latest reported construction of Ui0-66 is thin films on membrane substrates [118], [119]. The ever-evolving nature of UiO-66, combined with the possibility of generating new shapes by judiciously selecting a co-solvent was the final goal of this project.

Thus, the challenge in this experiment was to modify the morphology of UiO-66 using cosolvent glycols (EG, DEG, and TEG). This study was inspired by the reports of Cheng and Gaona-Gomez [86] who successfully tailored the shape of zeolite L(LTL) crystals using various glycols as an organic solvent in hydrothermal synthesis. The results showed transformation of crystals from disc like to rod like structures using TEG as a co-solvent assisting crystallization in the order $(\mathrm{TEG}>\mathrm{DEG}>\mathrm{EG})$.

In this study, the influence of different glycols on UiO-66 synthesized using Scheme $\mathbf{1}$ and Scheme 2 was unsuccessful as the crystals turned out to be amorphous. Hence, a new scheme following the published formula of Lu et al. [68] was modified as Scheme 3 with the addition of co-solvents to develop these UiO-66 crystals. 


\subsubsection{Results and Discussion}

The initiative of this experiment was to modify the morphology of UiO-66 using co-solvents, namely, EG, DEG, and TEG in the solvothermal synthesis process via Scheme 3 with a stir time of $24 \mathrm{~h}$ with subsequent heating at $120^{\circ} \mathrm{C}$ for $96 \mathrm{~h}$. The reactant concentrations and reaction conditions are summarized in Table 4.7.2-1. The as-synthesized sample mixture was also made using the same scheme with the above stated procedure without the addition of co-solvent. The as-synthesized sample is bolded for reference. The reactor was left for stirring for $24 \mathrm{~h}$, followed by heating at $120^{\circ} \mathrm{C}$ for $24 \mathrm{~h}$.

Table 4.7.2-1: Reactant composition and reaction conditions to investigate the effect of cosolvents.

\begin{tabular}{|c|c|c|c|c|c|c|c|c|}
\hline $\begin{array}{c}\mathrm{ZrCl}_{4} \\
\text { (mmol) }\end{array}$ & $\begin{array}{l}\text { 1,4-BDC } \\
\text { (mmol) }\end{array}$ & $\begin{array}{l}\text { DMF } \\
\text { (eq.) }\end{array}$ & $\begin{array}{c}\text { Acetic } \\
\text { Acid } \\
\text { (eq.) }\end{array}$ & $\begin{array}{l}\text { Co- } \\
\text { solve } \\
\text { nt }\end{array}$ & $\begin{array}{l}\text { Glycol } \\
\text { Eq. }\end{array}$ & $\begin{array}{c}\text { Stirring } \\
\text { time } \\
\text { (h) }\end{array}$ & $\begin{array}{c}\text { Temp } \\
\left({ }^{\circ} \mathrm{C}\right)\end{array}$ & $\begin{array}{l}\text { Reaction } \\
\text { time (h) }\end{array}$ \\
\hline 0.343 & 0.343 & 866 & 160 & - & 0 & 24 & 120 & 24 \\
\hline 0.343 & 0.343 & 866 & 160 & EG & 30 & 24 & 120 & 96 \\
\hline 0.343 & 0.343 & 866 & 160 & DEG & 30 & 24 & 120 & 96 \\
\hline 0.343 & 0.343 & 866 & 160 & TEG & 30 & 24 & 120 & 96 \\
\hline
\end{tabular}

The effect of various glycols was first experimented using Scheme 2 concentrations. The PXRD and SEM results revealed that both EG and DEG resulted in amorphous materials. The PXRD pattern showed a hump at lower $2 \theta$ angles while the rest of the pattern remained flat. The SEM images indicated large chunks of materials with grainy surface texture with no discrete particles. TEG on the other hand formed UiO-66 which was confirmed by comparing the peaks to the as-synthesized sample. However, these peaks were significantly broad with highly reduced intensities. The SEM image also reflected the poor crystallinity as the particles appeared as grains on a large surface. Some features also stood out from the mass 
signalling surface defects. The XRD and SEM results can be found in Appendix (Figure A.2-1 and Figure A.2-2). Thus, the synthesis recipe by Lu et al. [72] was adopted and modified with addition of glycols as Scheme 3. The as-synthesized sample for this section was also synthesized using Scheme 3, as per the method stated in the experimental procedure (Section 3.2.2) without the co-solvent using a stir time of $24 \mathrm{~h}$ heated at $120^{\circ} \mathrm{C}$ for $24 \mathrm{~h}$. All the samples in this experiment were characterized using PXRD, SEM; while FTIR, BET and TGA was performed on selected samples to gather additional structural information of unidentified phases.

The PXRD patterns with 30 eq. glycols produced extraordinary orientation modifications. Analyzing the diffractograms, from Figure 4.7.2-1, the as-synthesized sample of Ui0-66 was in excellent agreement with the literature (Figure 4.1.2-2). The addition of DEG and TEG as a co-solvent in the solvothermal synthesis of UiO-66 successfully produced pure phase UiO66 , identical to the as-synthesized sample. The sample with 30 eq. DEG showed a significant increase in peak intensity for the overall diffraction pattern, specially the ones corresponding to the angles $7.6^{\circ}, 8.6^{\circ}$, and $25.9^{\circ}$. The narrowing of peaks and rise in intensity clearly reflected higher phase purity and larger particles than the as-synthesized version of UiO-66 crystals. Similarly, the addition of 30 eq. TEG to the reaction mixture produced UiO66 with comparable peak intensities over the entire $2 \theta$ without peak splitting. This indicated comparable crystallinity as the as-synthesized UiO-66. Thus, the diffraction patterns attained for both TEG and DEG sample demonstrated increased degree of ordering of pure phase UiO-66 crystals in the order DEG >>TEG. 


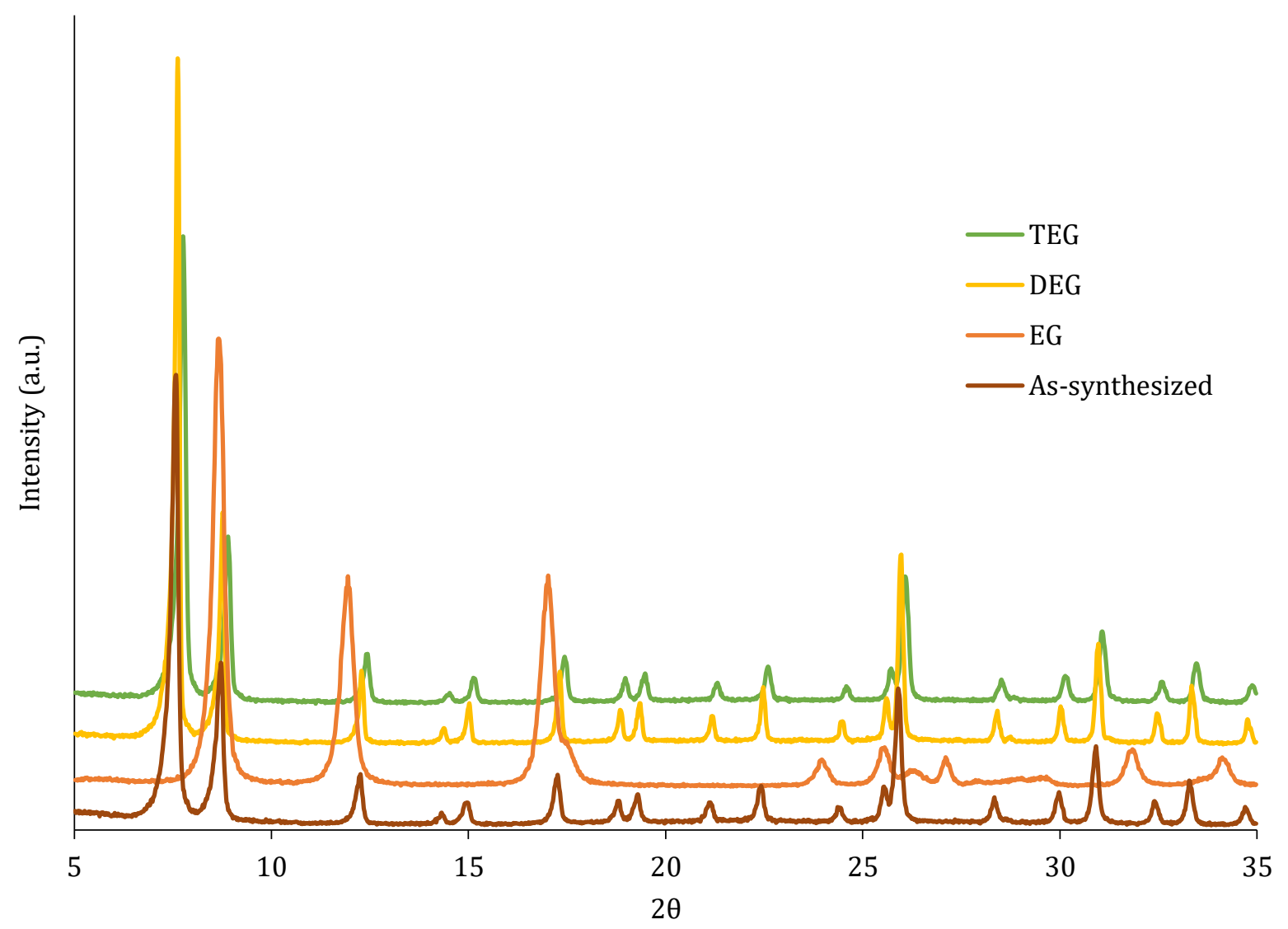

Figure 4.7.2-1: PXRD Pattern of samples produced with 30 eq. glycols.

The major contrast that appeared in the results was the sample synthesized with EG. In comparison to the as-synthesized sample, it was found that several peaks were absent while other peaks were exaggerated with the addition of $\mathrm{EG}$ as a co-solvent. A detailed comparison of the diffraction patterns for as-synthesized UiO-66 and the EG co-solvent synthesized UiO66 (named as EG-UiO-66), in Figure 4.7.2-2, showed a stark contrast. The first feature highlighted in this figure was the shift of the diffraction pattern to a lower angle signalling a significant increase in the pore size [38], [120]. The figure also presented systematic absence of peaks from the pattern corresponding to the Miller indices of [111], [311], [222], [331], [420], [333], and [440] while the peaks at the indices [200], [220], [400], [440], [600] at 8.68, $12,17,24,26^{\circ}$, respectively, strengthened in the presence of 30 eq. EG implying oriented evolution which was also observed for layered growth of UiO-66, and other zeolites [118], 
[119], [121]. As evident, the peaks significantly intensified in the directions (200), (220), and (400) as opposed to the (111) plane in as-synthesized sample.

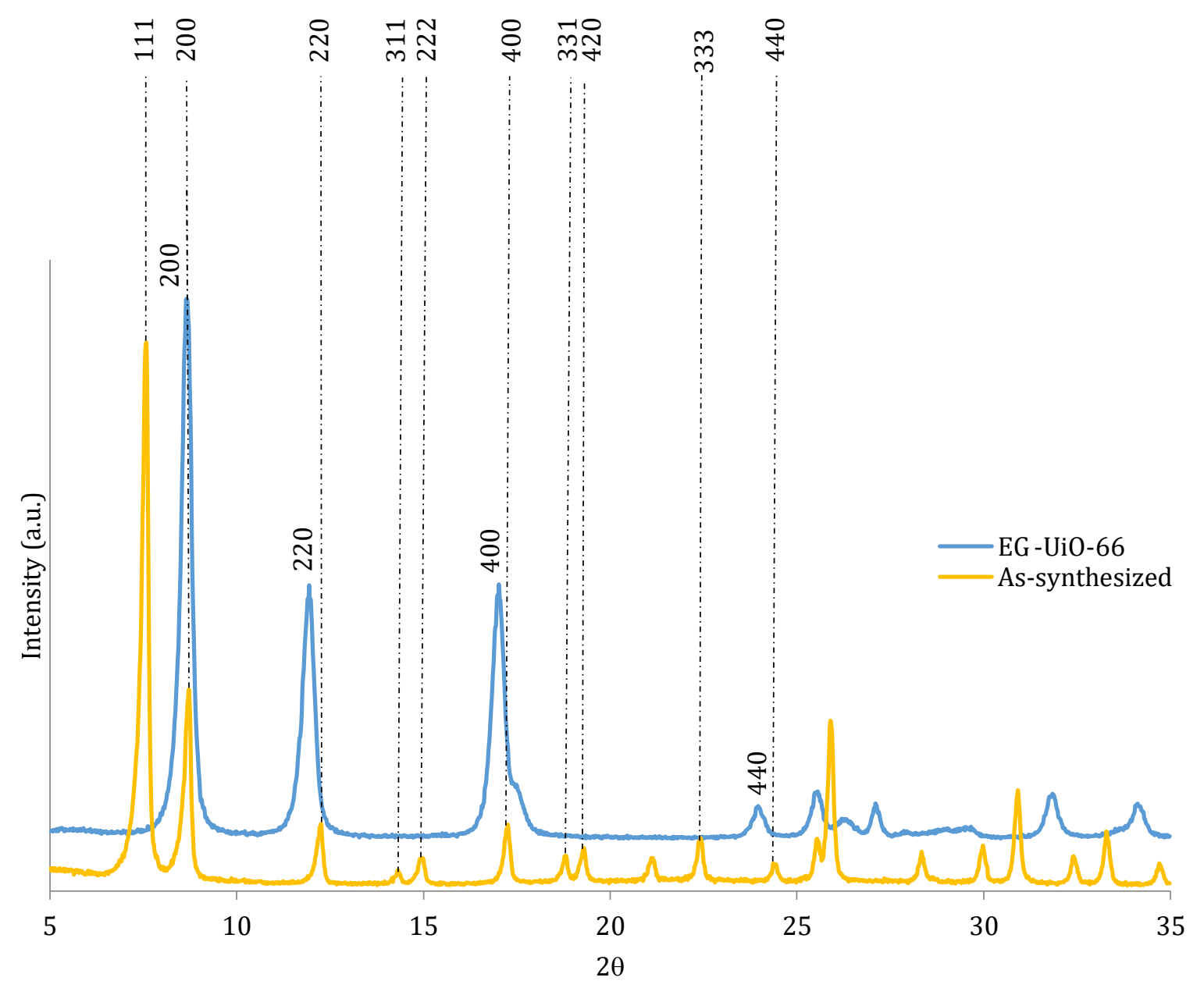

Figure 4.7.2-2: Miller indices of as-synthesized UiO-66 versus UiO-66 synthesized with EG as co-solvent.

The extent of directionality was evaluated using the crystallographic preferred orientation (CPO) [118] given by the equation 4.7.2-1, where I = intensity of reflections contributed by the EG-UiO-66 and as-synthesized samples in the planes $\mathrm{X}$ and $\mathrm{Y}$.

To conceptually confirm the observed phenomenon in EG-UiO-66, its distinctive peak at (200) was compared to the peaks at (111) and (220) using equation (4.7.2-1). 


$$
C P O \frac{X}{Y}=\frac{\frac{I_{A}^{X}}{I_{A}^{Y}}-\frac{I_{B}^{X}}{I_{B}^{Y}}}{\frac{I_{B}^{X}}{I_{B}^{Y}}}
$$

The respective peak intensities were normalized to calculate the CPO values in Table 4.7.2-2. The value was undefined for CPO (200/111) and 17 for CPO (200/220). The CPO value of (200/220) was certainly positive, remaining well above CPO $\geq 1$ which indicated that EG suppressed the growth rate of UiO-66 in (111) making (200) its preferred growth direction.

Table 4.7.2-2: Calculated $\mathrm{CPO}$ values based on reflections $\mathrm{X}$ and $\mathrm{Y}$.

\begin{tabular}{cc}
\hline Plane index Comparison $(\mathbf{X} / \mathbf{Y})$ & Calculated CPO \\
\hline$(200 / 111)$ & Undefined \\
$(200 / 220)$ & 17 \\
\hline
\end{tabular}

In addition, the SEM images, as depicted in Figure 4.7.2-3, revealed significantly transformed morphologies. The as-synthesized sample, as reported in the literature [72], produced sharp octahedral structures with a particle size of $0.337 \pm 0.055 \mu \mathrm{m}$; while the addition of 30 eq. DEG resulted in $3 \mathrm{x}$ larger monodisperse discrete rounded octahedral crystals of $0.97 \pm 0.092 \mu \mathrm{m}$ in size with rare appearance of small tumor growth on crystal surface. The large size difference indicated rigorous development in crystallinity. In addition, the narrow size distribution dictated that DEG may have slowed down the nucleation rate in such a way that nucleation was even throughout the solution causing uniform growth of large particles. A 30 eq. TEG, on the other hand, produced discrete polydisperse octahedral crystals with a particle size of $0.267 \pm 0.21 \mu \mathrm{m}$ which was rather comparable to the as-synthesized version. In addition, the TEG sample showed noticeable decline in homogeneity of the sample while the DEG sample showed a higher degree of monodispersity compared to the as-synthesized UiO-66 sample. In contrast, a 30 eq. EG as co-solvent promoted formation of spindle fibre like morphologies with roughly $0.8-1.0 \mu \mathrm{m}$ in length and $0.01 \mu \mathrm{m}$ in diameter. Thus, the SEM images were synonymous to the physical properties expressed in PXRD results. 


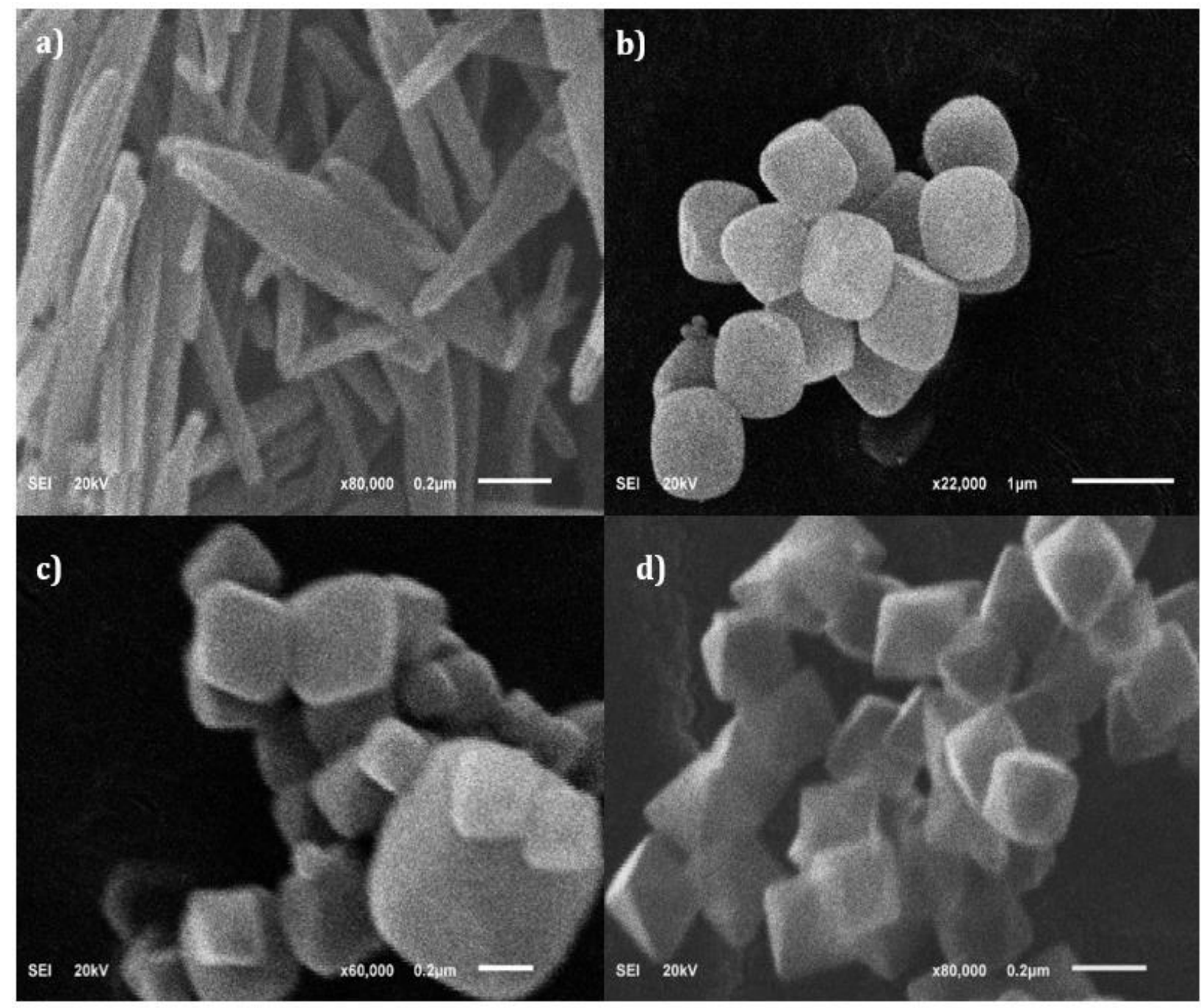

Figure 4.7.2-3: SEM images of samples produced with 30 eq. a)EG, b)DEG, c)TEG, and d)assynthesized (no co-solvent).

The FTIR spectroscopy was further performed to characterize the samples produced using EG, DEG and TEG as a co-solvent since they yielded promising results in enhancing and tuning the morphology of UiO-66. The results are presented in Figure 4.7.2-5 from a wavenumber range of $650 \mathrm{~cm}^{-1}$ to $4000 \mathrm{~cm}^{-1}$, however, a part of the spectrum is omitted as it remains mostly featureless at wavenumbers larger than $3000 \mathrm{~cm}^{-1}$. The as-synthesized sample (Figure 3.3.3-1) was found to be in good agreement with the literature characteristic vibrational modes presented in Table 4.7.2-3. The FTIR spectra of the as-synthesized sample 
was then compared to the glycol synthesized samples to reveal the underlying reasons that resulted in these unique morphologies.

Table 4.7.2-3: Vibrational modes of the IR Spectrum of UiO-66 reported in literature.

\begin{tabular}{|c|c|c|c|}
\hline $\begin{array}{c}\text { As- } \\
\text { synthesized } \\
\text { UiO-66 sample } \\
\left(\mathrm{cm}^{-1}\right) \\
\end{array}$ & Wavenumber $\left(\mathrm{cm}^{-1}\right)$ & Description & References \\
\hline 1665.38 & 1667 & DMF (amide group) & {$[41]$} \\
\hline 1589.9 & 1600 & $\begin{array}{c}\text { stretching vibrations of } \mathrm{C}=\mathrm{O} \text { in } \\
\text { the carboxylic acid present in } \\
\text { BDC }\end{array}$ & [122] \\
\hline 1505 & 1539 & CC ring & {$[41]$} \\
\hline 1391.93 & $\begin{array}{l}\text { Doublet at } 1589 \text { and } \\
1395\end{array}$ & $\begin{array}{l}\text { Carboxylate group (in- and out- } \\
\text { of-phase stretching mode) }\end{array}$ & [41] \\
\hline 1155.03 & 1367 & CC ring & {$[41]$} \\
\hline 1091.81 & 1085 & $\begin{array}{l}\mathrm{O}-\mathrm{C}-\mathrm{O} \text { asymmetric stretching } \\
\text { in the BDC ligand }\end{array}$ & [122] \\
\hline 818.31 & 814 & $\begin{array}{c}\mathrm{OH} \text { bending }+\mathrm{CH} \text { bending (anti- } \\
\text { phase) }\end{array}$ & {$[41]$} \\
\hline 743.12 & 745 & Zr-carboxylate group bond & {$[123]$} \\
\hline 670 & 673 & $\mu_{3}-\mathrm{O}$ stretching & {$[41]$} \\
\hline
\end{tabular}

A summary of the effect of 30 eq. EG, DEG, and TEG on the vibrational characteristics of various molecules of synthesized UiO-66 samples is depicted in Figure 4.7.2-5 and a comparison between their vibrational modes $\left(\mathrm{cm}^{-1}\right)$ is presented in Table 4.7.2-4. 


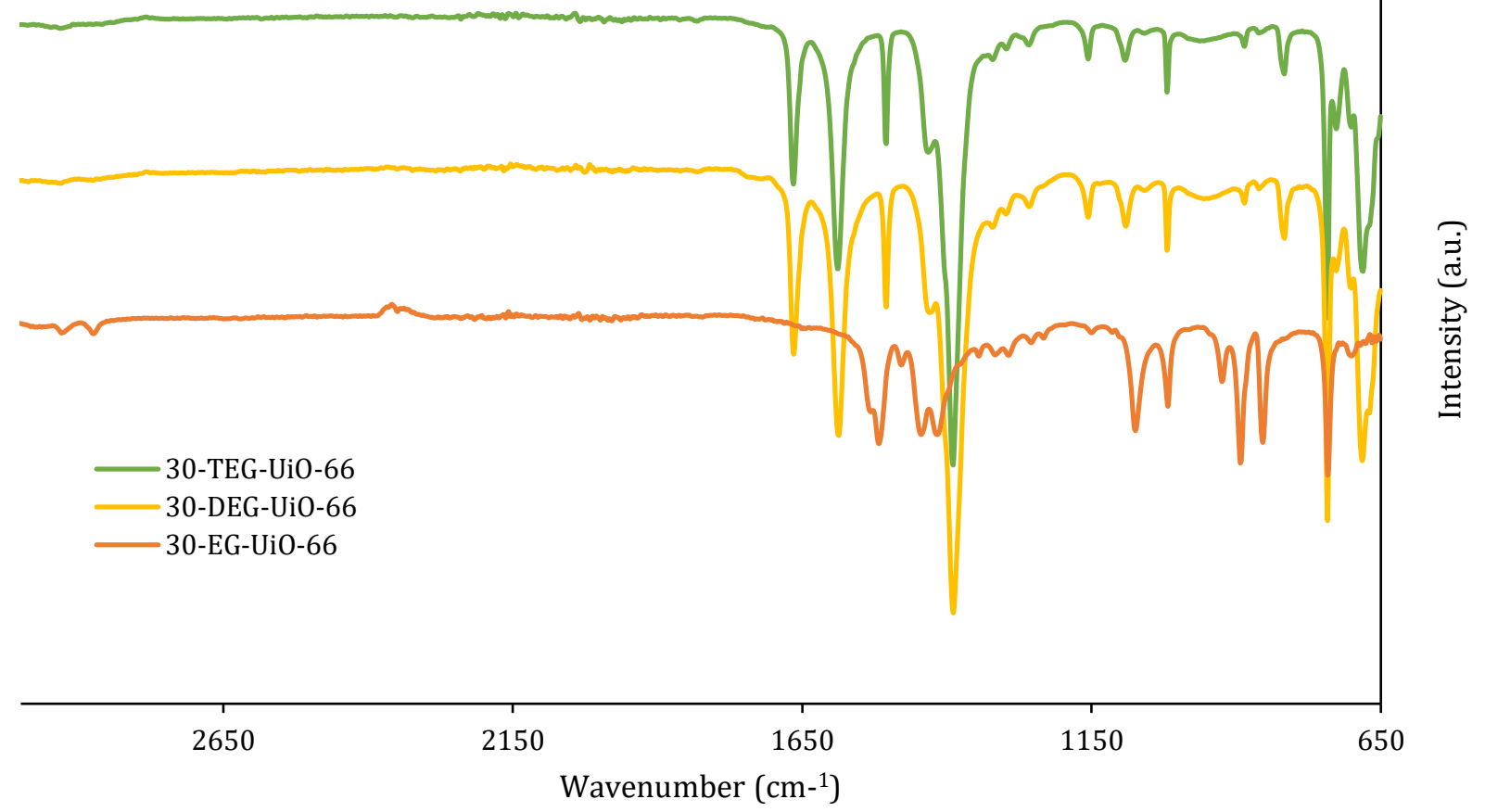

Figure 4.7.2-4: FTIR spectrum reported on the same wavenumber range for UiO-66 samples synthesized using co-solvent EG, DEG, and TEG. Scaling of the spectra has been omitted for clarity.

Table 4.7.2-4: A comparison of the vibrational modes $\left(\mathrm{cm}^{-1}\right)$ for the UiO-66 samples synthesized to examine the effect of glycolsa.

\begin{tabular}{|c|c|c|c|c|}
\hline $\begin{array}{c}\text { As- } \\
\text { synthesized } \\
\text { Ui0-66 } \\
\end{array}$ & $\begin{array}{c}\text { 30-TEG-UiO- } \\
66\end{array}$ & $\begin{array}{l}\text { 30-DEG-UiO- } \\
66\end{array}$ & $\begin{array}{c}\text { 30-EG-UiO- } \\
66\end{array}$ & Description \\
\hline- & - & - & 2926,2873 & BHET (OH group) [61] \\
\hline 1665.38 & 1665.36 & 1664.98 & - & DMF (amide group) \\
\hline 1589.9 & 1589.3 & 1588.24 & - & $\begin{array}{c}\text { BDC (O-C-O asymmetric } \\
\text { stretching) [122] }\end{array}$ \\
\hline 1505.04 & 1505.18 & 1504.7 & 1517.4 & $\begin{array}{c}\mathrm{BDC}(\mathrm{C}=\mathrm{C} \text { of benzene } \\
\text { rings })[122]\end{array}$ \\
\hline- & - & - & 1460 & $\begin{array}{c}\text { BHET }\left(\mathrm{CH}_{2} \text { bending) }\right. \\
{[124]}\end{array}$ \\
\hline- & - & - & $\begin{array}{l}1429.28 \\
1416.59\end{array}$ & $\begin{array}{l}\text { BHET (terminal OH } \\
\text { group of carboxylic } \\
\text { acid) [61] }\end{array}$ \\
\hline
\end{tabular}




\begin{tabular}{|c|c|c|c|c|}
\hline $\begin{array}{c}\text { As- } \\
\text { synthesized } \\
\text { Ui0-66 }\end{array}$ & $\begin{array}{l}\text { 30-TEG-UiO- } \\
66\end{array}$ & $\begin{array}{c}\text { 30-DEG-UiO- } \\
66\end{array}$ & $\begin{array}{c}\text { 30-EG-UiO- } \\
66\end{array}$ & Description \\
\hline 1391.93 & 1391.83 & 1391.024 & - & $\begin{array}{l}\text { O-C-0 symmetric } \\
\text { stretching [122] }\end{array}$ \\
\hline 1155.03 & 1155.22 & 1155.09 & 1148.36 & $\begin{array}{c}\operatorname{DMF}\left(\rho \mathrm{C}^{\prime} \mathrm{H}_{3}+\rho \mathrm{C}^{\prime \prime} \mathrm{H}_{3}\right) \\
{[125]}\end{array}$ \\
\hline 1091.81 & 1091.35 & 1090.96 & - & $\begin{array}{c}\mathrm{DMF}\left(\rho \mathrm{C}^{\prime} \mathrm{H}_{3}+v \mathrm{CN}\right) \\
{[125]}\end{array}$ \\
\hline - & - & - & 1071.21 & $\begin{array}{c}\text { BHET (C=0 stretching) } \\
{[124]}\end{array}$ \\
\hline 1019.19 & 1019.13 & 1019.1 & 1017.86 & BDC $[122]$ \\
\hline- & - & 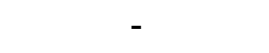 & 924.26 & $\mathrm{NH}_{3}[126]$ \\
\hline- & - & - & 892.3 & $\begin{array}{c}\text { BHET or EG ( } \mathrm{CH}_{2} \\
\text { rocking) [124] }\end{array}$ \\
\hline- & - & - & 853.81 & $\begin{array}{c}\text { BHET (vC-C stretching) } \\
{[61]}\end{array}$ \\
\hline 818.31 & 816.52 & 816.52 & - & $\begin{array}{c}\text { OH bending }+\mathrm{CH} \\
\text { bending (anti-phase) } \\
{[41]}\end{array}$ \\
\hline 743.12 & 742.95 & 743.04 & 741 & $\begin{array}{l}\text { Zr-carboxylate group } \\
\text { bond [123] }\end{array}$ \\
\hline 670.1 & 678 & 682 & - & $\mu_{3}-0$ stretching [41] \\
\hline
\end{tabular}

a See Figure 4.7.2-4 for a pictorial representation of the IR spectra of UiO-66 samples synthesized with co-solvent glycols.

The IR spectra of the samples with the closest PXRD and SEM results to the as-synthesized UiO-66 were first compared to detect the initial changes in the morphology, i.e. with 30 eq. TEG and DEG. As seen from Figure 4.7.2-4 and Table 4.7.2-3, most of the vibrational modes in the sample synthesized with TEG and DEG remained the same in comparison to the assynthesized UiO-66 contributing from the bonds stated in the description. Both samples showed comparable intensities for the identified peaks except that a major shift was observed for the vibrational frequency relating to the $\mathrm{Zr}$-carboxylate bond that connected the metal cluster and the carboxylate linker group. The effect of TEG sample showed a shift in the vibrational frequency from $678 \mathrm{~cm}^{-1}$ to a higher frequency of $678 \mathrm{~cm}^{-1}$, while the DEG sample showed a larger shift to $682 \mathrm{~cm}^{-1}$. The relevant shift in the FTIR spectra to a higher wavenumber was related to a higher interaction between the metal cluster and carboxylate group that stabilized this bond [127], which was evidently higher for the DEG sample. 
The expression of higher hydrophobicity coming from the bulkier alkyl group attached to the TEG may have caused poor interactions of the carboxylate group with the zirconium cluster leading to defined but polydisperse UiO-66 crystals. Similarly, using DEG as a co-solvent allowed a relatively stable interaction of the linker molecules with the zirconium metal center and also facilitated stronger interactions of the $\mathrm{Zr}$ and $\mathrm{O}$ atoms of the carboxylate group increased the lability of the framework in general which regulated the growth of the molecule. Thus, DEG may have affected the crystallization rate which promoted the phase purity of UiO-66 leading to large crystals. Additionally, the vibrational modes of the IR spectrum revealed a minor shift to the lower vibrational frequency corresponding to the $818.31 \mathrm{~cm}^{-1}$ of the as-synthesized Ui0-66 sample. This indicated better linkage between the $\mathrm{OH}$ and $\mathrm{CH}$ groups which may have been contributed by the respective glycols as a co-solvent in the reaction. Nevertheless, this assumption would need further quantitative elemental analysis.

Furthermore, from Figure 4.7.2-4 and Table 4.7.2-4, the vibrational modes of the substantially different IR spectrum of 30-EG-UiO-66 to that of the as-synthesized sample was correlated to the unique characteristics revealed by the PXRD and SEM images. Evidently, the peaks corresponding to the as-synthesized sample at $1665.38 \mathrm{~cm}^{-1}$ and $1589.9 \mathrm{~cm}^{-1}$ completely disappeared while the peak at $1505.04 \mathrm{~cm}^{-1}$ shifted to a higher vibrational frequency at $1517.4 \mathrm{~cm}^{-1}$. This shift was associated to stabilizing the $\mathrm{C}=\mathrm{C}$ bonds as a result of the increased interactions of these molecules within the framework. The peaks related with the wavenumbers $1460 \mathrm{~cm}^{-1}, 1429.28 \mathrm{~cm}^{-1}, 1416.59 \mathrm{~cm}^{-1}$, which were contributed by the $\mathrm{CH}_{2}$ bending and terminal $\mathrm{OH}$ group of carboxylic acid, were absent in the rest of the samples. These bands were ascribed to BHET due to the highly possible interactions between the EG and BDC molecules to form polyethylene terephthalate. Some additional peaks also appeared at $1074.21 \mathrm{~cm}^{-1}, 892.3 \mathrm{~cm}^{-1}$, and $853.81 \mathrm{~cm}^{-1}$ which were attributed to $\mathrm{C}=0$ stretching, $\mathrm{CH}_{2}$ rocking, and C-C stretching, respectively. The presence of BDC and zirconium within the framework was confirmed by the vibrational modes at $1017.86 \mathrm{~cm}^{-1}, 741.96 \mathrm{~cm}^{-1}$, respectively. The $\mathrm{Zr}-\mathrm{O}$ bond vibrational frequency was much lower than the literature value which indicated decreasing bond strength. Another prime feature of this spectrum was the 
missing $\mu_{3}-0$ stretching band corresponding to $670 \mathrm{~cm}^{-1}$. The absence of this band within the molecule indicated a different ligand that bonded the metal clusters in the framework.

The existence of new FTIR peaks in the 30 -eq. EG sample suggested that there were different interactions due to the introduction of new co-solvent in the reaction mixture. One possibility of the redirection of UiO-66 orientation from (111) (Figure 4.7.2-5b) plane to (Figure 4.7.2-5c) (200) was correlated to the crystal evolutionary theory [124] which was highlighted in UiO-66 films produced on substrate $\alpha-\mathrm{Al}_{2} \mathrm{O}_{3}$ where the associates grew in the direction parallel to the substrate with highest growth rate in the direction of (200) plane and a slower growth rate in (111) direction [123], [124]; contrast to this experiment where EG completely suppressed the growth of UiO-66 along (111) direction. If the EG molecules acted as a modulator, the solution would have increased concentration of competitive ligands that regulate the reaction equilibrium. As the ligand locations are labile points (Figure 4.7.2-5a) within the framework, the competitive nature of EG may have caused the replacement of ligands by EG molecules occupying certain regions within the framework of UiO-66. Thus, due to regulation of the coordination equilibrium in the thermodynamically driven reaction, it was speculated that EG may have successfully been incorporated into the microstructure leading to anisotropic crystals in the (200) direction without the use of a substrate. In support of this argument, it was found that the peaks at wavenumber 1074.21 $\mathrm{cm}^{-1}$ and $892.3 \mathrm{~cm}^{-1}$ belonging to $\mathrm{C}=\mathrm{O}$ stretching and $\mathrm{CH}_{2}$ rocking of BHET.

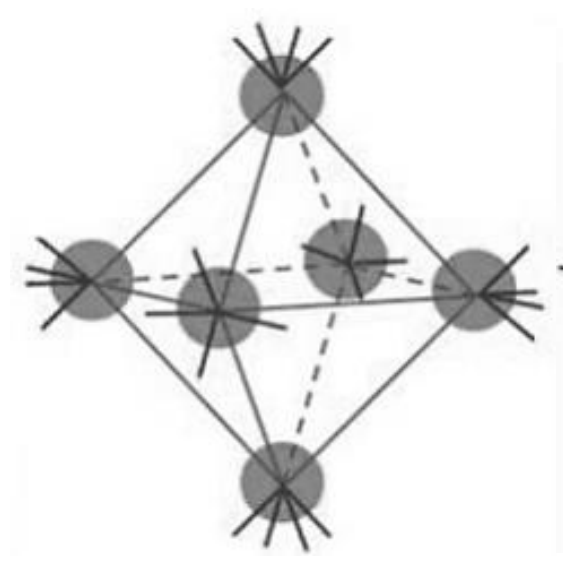

a)

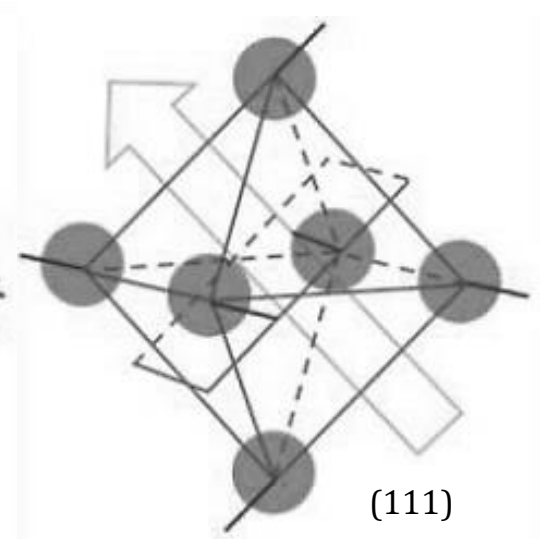

b)

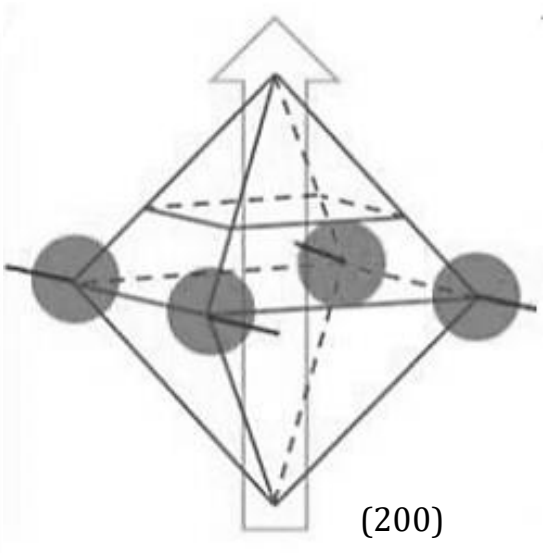

c)

Figure 4.7.2-5: Schematic representation of a) thermodynamically labile regions of UiO-66 b) configuration of UiO-66 in (111) plane c) configuration of UiO-66 in (200) plane [119]. 
Contrast to this theory, there may have been possible interactions of EG with DMF, BDC, acetic acid and among each other within the solution. By the conventional solvothermal synthesis route, the amine produced during thermal decomposition of DMF [128] initiates the deprotonation of the BDC linker, while acetic acid competes with the linker and regulates the attachment of linker to the zirconium clusters [4]. The FTIR spectrum from both DEG and TEG aided sample accounted for these interactions which were initially matched with the EG synthesized Ui0-66 sample. The remaining peaks were determined by shortlisting the interactions, out of which, the only possible interactions were EG with BDC or acetic acid [129], [130].

Firstly, as EG was added consecutively into the DMF before all other reactants, the high $\mathrm{p} K_{\mathrm{a}}$ value of EG (14.22) led to an instant increase in the basicity of the solution. The high $\mathrm{pH}$ of the solution being higher than the $\mathrm{p} K_{\mathrm{a}}$ of DMF (6.7) caused the DMF to readily protonate in the solution. Characterized by the rich protonated solution, the acetic acid deprotonated in amounts more than the conventional DMF solution.

The reaction between EG and acetic acid usually produces ethylene glycol monoacetate and diacetate but in the presence of a catalyst [131]; thus, this possibility was ruled out. The other possible interaction is between EG and BDC in the presence of protons which was deemed as a high possibility as the indirect effect of EG increased the protons within the solution. Thus, the high availability of protons facilitated a reaction between the EG and BDC, producing a monomer called bis(2-hydroxyethyl) terephthalate (BHET). Two peaks corresponding to $1074.21 \mathrm{~cm}^{-1}$ and $892.3 \mathrm{~cm}^{-1}$ were initially ascribed to both EG and BHET, however, the presence of $2926 \mathrm{~cm}^{-1}, 2873 \mathrm{~cm}^{-1}$ were exclusive to the existence of BHET in the sample. Thus, it was confirmed that BHET was an important part of the oriented UiO-66 framework. Hence, the new peaks in the EG synthesized UiO-66 sample were matched with the probable reaction, revealing the existence of BHET in the reoriented UiO-66 framework.

There have been several reports on the formation of in-situ ligand in the literature, such as the case of Mn-MOFs synthesized with different in-situ ligands [132]. In this case, changing the ligand synthesized 3 new coordination compounds. The difference in structures 
stemmed from the different ligating sites of the in-situ ligands which in turn affected the overall packing structure of the Mn-clusters and nuclearities, thereof. Thus, the oriented growth of UiO-66 with 30-eq. EG was credited to the interaction of EG with other components within the reaction to generate an in-situ ligand that promoted parallel growth of UiO-66 crystals in the (200) direction, while completely eliminating their growth rate in the (111) direction. The schematic of this reaction mechanism is presented in Figure 4.7.2-6. The reaction was obvious with their respective $\mathrm{p} K_{\mathrm{a}}$ values of $\mathrm{EG}=14.22$ [133] and terephthalic acid $=3.32[134]$ which necessitates a condensation reaction between EG and BDC in the presence of $\mathrm{H}^{+}$protons generated by the simultaneous deprotonation of BDC to form the monomer (BHET) bis(2-hydroxyethyl) terephthalate [129], [130]. This compound became an in-situ ligand connecting the zirconium metal clusters to form fibrous UiO-66 crystalline materials. 


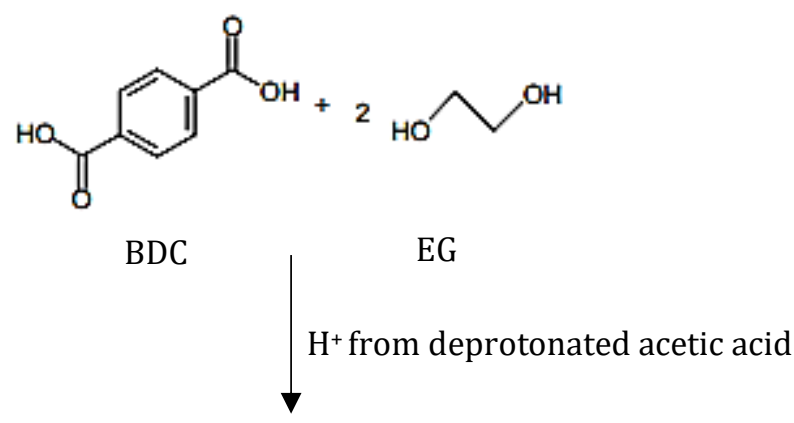

In-situ ligand formation by condensation reaction

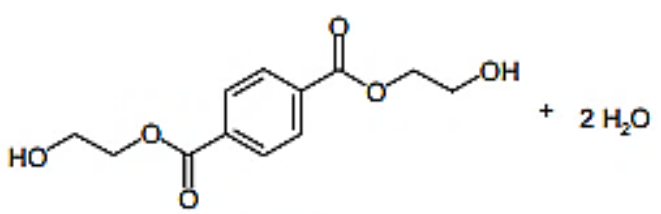

BHET ligand monomer

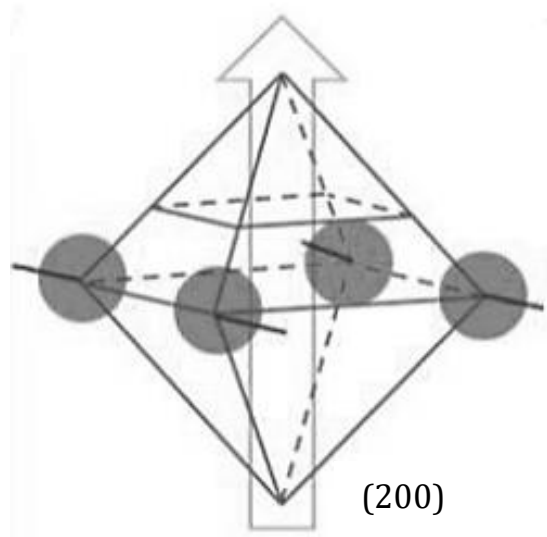

30-EG-UiO-66

Figure 4.7.2-6: Illustration of the production of $i n$-situ ligand BHET and subsequent oriented growth of UiO-66 crystals in the (200) with 30-eq. EG co-solvent.

Following the FTIR results, the surface area of the 30 -EG-UiO- 66 was measured by $\mathrm{N}_{2}$ adsorption as shown in Figure 4.7.2-7. The BET surface area analysis of the sample resulted to be a value of $88.0850 \mathrm{~m}^{2} / \mathrm{g}$, along with an interparticle void of $114.44 \AA$, and pore volume $0.25 \mathrm{~cm}^{3} / \mathrm{g}$. Evidently, the adsorption isotherm for this sample adsorbed limited quantity of nitrogen gas even at increased applied pressure. This was a possible indication of blocked pores within the sample; however, the material had still managed to adsorb at higher relative pressure of at most $220 \mathrm{~cm}^{3} / \mathrm{g}$ of $\mathrm{N}_{2}$. The desorption curve showed the $\mathrm{N}_{2}$ molecules 
remained within the framework on decreasing the pressure. This occurrence may have been due to the bulky in-situ ligand formation incorporating the UiO-66 framework. Additionally, this observation is perfectly in line with the PXRD observation which indicated reduction in pore size of the UiO-66 crystals.

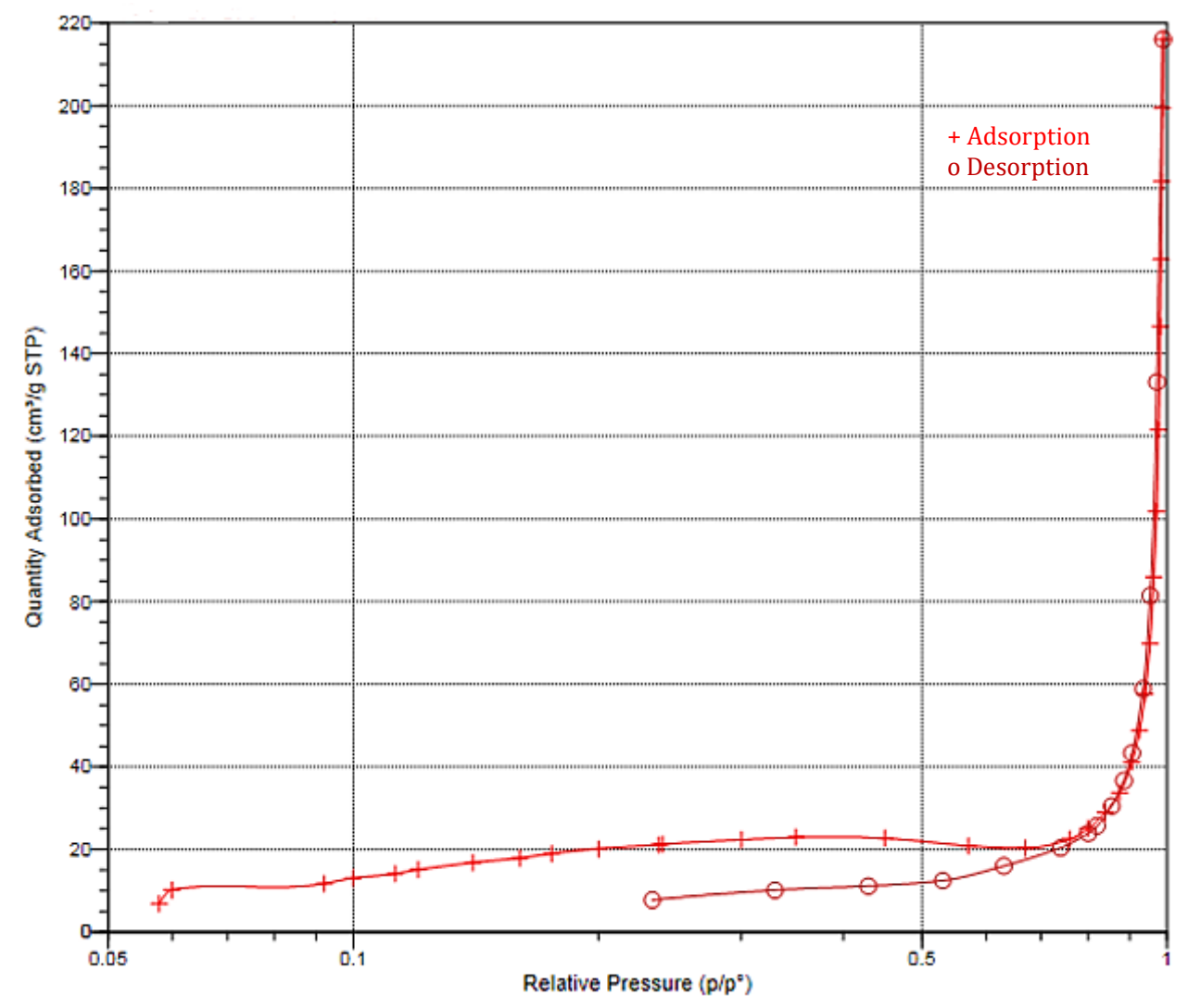

Figure 4.7.2-7: Adsorption isotherm log plot of 30-EG-UiO-66.

In addition, a TGA analysis was also performed on this sample to compare the framework stability with the as-synthesized UiO-66. The TGA of the as-synthesized sample is depicted in Figure 3.3.4-1.

The weight loss expressed in the TGA of as-synthesized UiO-66 (Figure 3.3.4-1) was in good agreement with the corresponding temperatures in literature (Figure 2.3.2-1b). The first weight loss in the TGA graph was attributed to the physiosorbed water molecules in the framework accounting to $3.5 \%$ of the sample weight. The second weight loss was the evaporation of DMF solvent from the pores of the material with complete removal at $500^{\circ} \mathrm{C}$ and accounted for a 9.5\% weight. The desolvated and dehydrated clusters contain a total of 
$13 \%$ weight of the UiO-66 initial structure. Further heating of the sample led to loss of BDC linker molecules leaving behind a residue of $\mathrm{ZrO}_{2}$. The expected weight loss for the last step was $54.6 \%$ [46], while the experimental results were calculated to be $47.5 \%$. The decrease in the weight loss percentage indicated that the as-synthesized sample in this experiment was slightly defective than the literature reported UiO-66 samples because of missing linker that connected the Zr-clusters.

The TGA analysis of the as-synthesized sample was cross-examined with the TGA of the 30EG-UiO-66 sample which is represented in Figure 4.7.2-8. The TGA graph displayed a significant contrast to the as-synthesized sample. The sample experienced sluggish weight loss of $3 \%$ until $365^{\circ} \mathrm{C}$. The first major step was observed between $365^{\circ} \mathrm{C}$ and $490^{\circ} \mathrm{C}$ with a $9.5 \%$ weight loss. The second one was a consecutive occurrence noticed between $490^{\circ} \mathrm{C}$ and $600^{\circ} \mathrm{C}$ with a weight loss of $34.5 \%$. The last change observed was a small slope between $600^{\circ} \mathrm{C}$ and $1000^{\circ} \mathrm{C}$ of $11.5 \%$ weight loss. It was difficult to predict the elements involved in various stages of weight losses in the TGA for this sample; however, the total weight loss\% was $58.5 \%$ of the original weight which indicated higher thermal stability than the assynthesized sample. Thus, an elemental composition analysis is required for the newly synthesized needle-shaped UiO-66 crystals. 


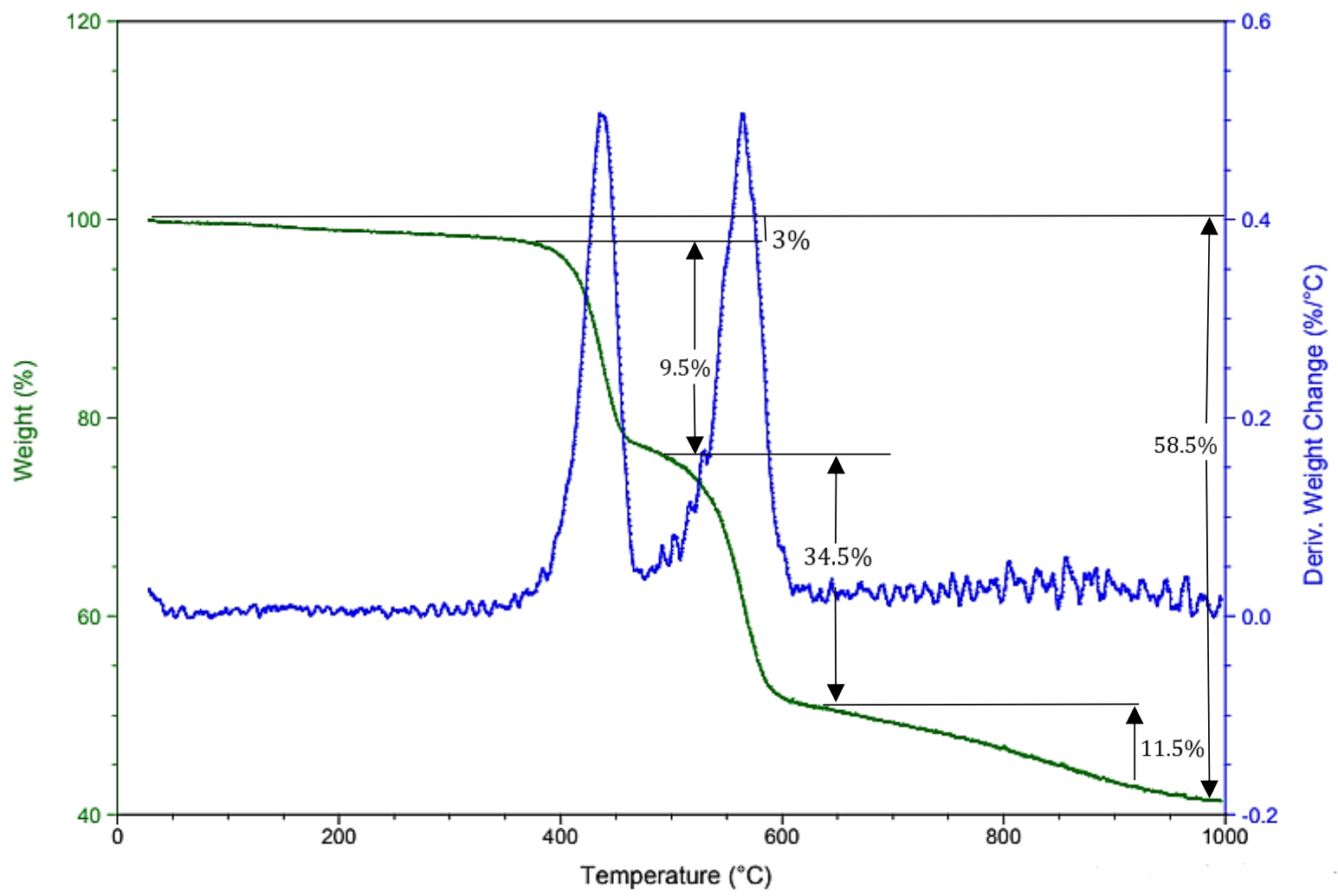

Figure 4.7.2-8: TGA(green) and DSC(blue) profiles of 30-EG-UiO-66.

For the effect of DEG on the morphology of UiO-66, as DEG was added into the solution consecutively into the DMF before all other reactants, the basicity of the solution increased due to the high $\mathrm{p} K_{\mathrm{a}}$ value of DEG (14.2). The $\mathrm{pH}$ of the solution being higher than the $\mathrm{p} K_{\mathrm{a}}$ of DMF (6.7) caused the DMF to readily protonate in the solution. Characterized by the rich protonated solution, the acetic acid deprotonated in amounts more than the conventional DMF solution. The BDC linker being slightly higher in the $\mathrm{p} K_{\mathrm{a}}$ scale than acetic acid (3.51>4.82) "won" over the bond formation between $\mathrm{Zr}$ atom and the BDC linkers making the SBUs more acetate ion and $\mathrm{Zr}$ bond oriented, if not completely replacing the linker. Moreover, the incorporation of acetate ions into the framework slowed down the nucleation rate further, resulting in increased particle size [135], [136]. Also, the high acetate components in the UiO-66 framework, from literature, increased the number of defect sites as they served to compensate the charges of the missing linker. This necessitates the creation of missing linker and clusters in the structure of UiO-66 that in turn produced large octahedral Ui0-66 crystals [112]. The assumption of this dynamic is proposed based on the 
fact that the strength of a metal-ligand bond increases as the basicity of the ligand increases [137], [138]. Thus, introducing DEG into the solution amplified the availability of deprotonated acetate molecules in the solution. As the acetate molecules had a better chance of bonding with the $\mathrm{Zr}$ atoms, justified by the $\mathrm{p} K_{\mathrm{a}}$ values, the SBUs grew in that fashion incorporating more acetate instead of the carboxylate group of BDC, making the subsequent UiO-66 crystals larger in size.

Unlike the effect of co-solvent DEG, the incorporation of TEG as a co-solvent in the reaction mixture proved to be a disadvantage in the formation of UiO-66 crystals. The surface defects and increased non-uniformity of the particles were sustained at all concentrations of TEG. Relating to the $\mathrm{pH}$ and $\mathrm{p} K_{\mathrm{a}}$ working dynamic introduced for the effect of DEG, the $\mathrm{p} K_{\mathrm{a}}$ of DEG and TEG are nearly equal in value, 14.2 and 14.5, respectively. The values necessitated the same behavior of increasing the $\mathrm{pH}$ of the solution (higher than the $\mathrm{p} K_{\mathrm{a}}$ of DMF (6.7)) protonated the DMF molecules. Due to the high $\mathrm{p} K_{\mathrm{a}}$ value of TEG (14.5), the introduction of TEG also increased the basicity of the solution. Thus, the basicity of the solution caused deprotonation of the acetate molecules which overcame the concentration of deprotonated BDC becoming integrated within the UiO-66 SBU instead of BDC. The competition between these molecules slowed the conventionally fast nucleation rate yielding particles higher in surface area which was evident from the SEM images of the UiO-66 crystals. Also, the habit of acetate components to compensate the charges of the missing linker increased the number of both missing linkers and missing clusters that created larger pores than usual [112]. Thus, the integration of acetate molecules in the framework produced large octahedral UiO-66 crystals [112]. However, the particles were polydisperse compared to the UiO-66 crystals produced from the DEG co-solvent. As the difference in $\mathrm{p} K_{\mathrm{a}}$ values of both TEG and DEG were negligible, the other significant difference was in their respective structure with the increased bulky alkyl groups in TEG. Thus, we predicted that although the higher TEG content was sufficient in increasing the particle size of the UiO-66 crystals, the longer alkyl chain length hindered the successful incorporation of acetic acid into the SBU. This claim is further justified by the difference in viscosities in each of these co-solvents. The viscosity increases with the increase in alkyl chain length as $20 \mathrm{cP}, 35.7 \mathrm{cP}$, and 49, cP for EG, DEG, and TEG, respectively. Thus, it becomes more difficult for TEG to extend the octahedral 
framework as the high viscosity surrounding the nucleation sites hinder the connection of BDC with the central $\mathrm{Zr}$ atoms. As a result, the resultant UiO-66 crystals always were polydisperse in nature.

\subsubsection{Conclusions}

To conclude, Ui0-66 morphology was successfully modified using 30-eq. glycols (EG, DEG, and TEG). DEG served in enhancing the octahedral morphology with higher crystallinity in the (111) plane and particle size of $0.97 \pm 0.092 \mu \mathrm{m}$ which was three times larger than the traditional particle size. Apparently, DEG indirectly acted as a strong structure directing agent which slowed down the nucleation rate of the crystals. As a result, large octahedral crystals were produced in the process. TEG, in contrast, produced polydisperse octahedral shapes with comparable crystallinity and a particle size of $0.267 \pm 0.21 \mu \mathrm{m}$. This may have been because of the relatively bulkier alkyl chains attached to the molecule which disabled strong competition with the BDC molecules ensuing equivalent results as the as synthesized sample.

A clear distinction was observed with the use of EG which transformed the morphology from octahedral crystals to anisotropic fibre-like structures. The FTIR of the EG aided synthesis of UiO-66 showed increased activity between $\mathrm{C}=\mathrm{C}$ bond of benzene rings, along with additional peaks which confirmed the presence of newly synthesized ligand BHET. The production of in-situ ligand led to oriented growth of UiO-66 in the (200) direction. However, the BET surface analysis reported a value of $88.0850 \mathrm{~m}^{2} / \mathrm{g}$ which was 12 times lower than the literature value [37]. Comparing this result to the PXRD and SEM results lead to a conclusion that although the material was microporous, the pores of the framework may have been blocked because of the lengthy and bulky ligand. Additionally, the observations suggested a strong dependency of UiO-66 morphology on the alkyl chain length of glycols where, increased chain length posed a possible blockage of pores within the framework. 


\subsection{EFFECT OF THE CONCENTRATION OF GLYCOLS}

\subsubsection{ETHYLENE GLYCOL}

\subsubsection{Results and Discussion}

As established in Section 4.7, the change of concentration in modulator or solvent had a pronounced effect on the morphology of Ui0-66 and other MOFs as well. Thus, the results from Section 4.7 using EG as a co-solvent led to the hypothesis that the concentration of EG would have a significant effect on the directional morphology of UiO-66, which was explored in this section. Henceforth, the main idea of this experiment was to detect the critical concentration of EG required to cause morphological transformation of UiO-66. The samples were synthesized with EG as a co-solvent in the solvothermal synthesis process of UiO-66 via Scheme 3 with a stir time of $24 \mathrm{~h}$ with subsequent heating at $120^{\circ} \mathrm{C}$ for $96 \mathrm{~h}$. The reactant concentrations and reaction conditions are summarized in Table 4.8.1.1-1. The assynthesized sample was generated without the co-solvent with a stir time of $24 \mathrm{~h}$ which was subsequently heated at $120^{\circ} \mathrm{C}$ for $24 \mathrm{~h}$ and is bolded for reference.

Table 4.8.1.1-1: Reactant composition and reaction conditions to investigate the effect of EG concentration.

\begin{tabular}{cccccccc}
\hline $\begin{array}{c}\mathrm{ZrCl}_{4} \\
(\mathbf{m m o l})\end{array}$ & $\begin{array}{c}\mathbf{1 , 4 - B D C} \\
(\mathbf{m m o l})\end{array}$ & $\begin{array}{c}\text { DMF } \\
(\mathbf{e q})\end{array}$ & $\begin{array}{c}\text { Acetic } \\
\text { Acid } \\
\text { (eq.) }\end{array}$ & $\begin{array}{c}\text { Glycol } \\
\mathbf{E q} .\end{array}$ & $\begin{array}{c}\text { Stirring } \\
\text { time } \\
\text { (h) }\end{array}$ & $\begin{array}{c}\text { Temperature } \\
\left({ }^{\circ} \mathbf{C}\right)\end{array}$ & $\begin{array}{c}\text { Reaction } \\
\text { time (h) }\end{array}$ \\
\hline $\mathbf{0 . 3 4 3}$ & $\mathbf{0 . 3 4 3}$ & $\mathbf{8 6 6}$ & $\mathbf{1 6 0}$ & $\mathbf{0}$ & $\mathbf{2 4}$ & $\mathbf{1 2 0}$ & $\mathbf{2 4}$ \\
0.343 & 0.343 & 866 & 160 & 10 & 24 & 120 & 96 \\
0.343 & 0.343 & 866 & 160 & 20 & 24 & 120 & 96 \\
0.343 & 0.343 & 866 & 160 & 30 & 24 & 120 & 96 \\
\hline
\end{tabular}


The XRD diffractograms for the synthesized samples are displayed in Figure 4.8.1.1-1. It was observed that on increasing the concentration of EG from 5 to 10 eq. the XRD pattern showed no noticeable peak shift. The peak indices matched the XRD pattern of the published UiO-66 crystals with the only difference of significantly lowered intensities and peak broadening which indicated reduction in crystallinity of the octahedral particles. Furthermore, starting from 20 eq. EG, a large shift to the higher angle was observed in the XRD pattern. The peaks corresponding to $2 \theta$ angle $7.5^{\circ}$, associated with the plane (111) of the EG samples, diminished while the peaks at $8.7^{\circ}$ and $12^{\circ}$, associated with the plane (200) and (220), respectively, greatly intensified. The intensifying of peaks associated with (200) and (220) planes indicated that the direction of growth had changed with the added EG concentration. Nonetheless, this rise only lasted up to 30 eq. concentration of EG for highest peak intensities in the direction (200) and (220). The addition of 40 and 50 eq. amounts of EG resulted in humped diffraction patterns indicating degradation in the crystallinity of the material, or rather amorphous materials. 


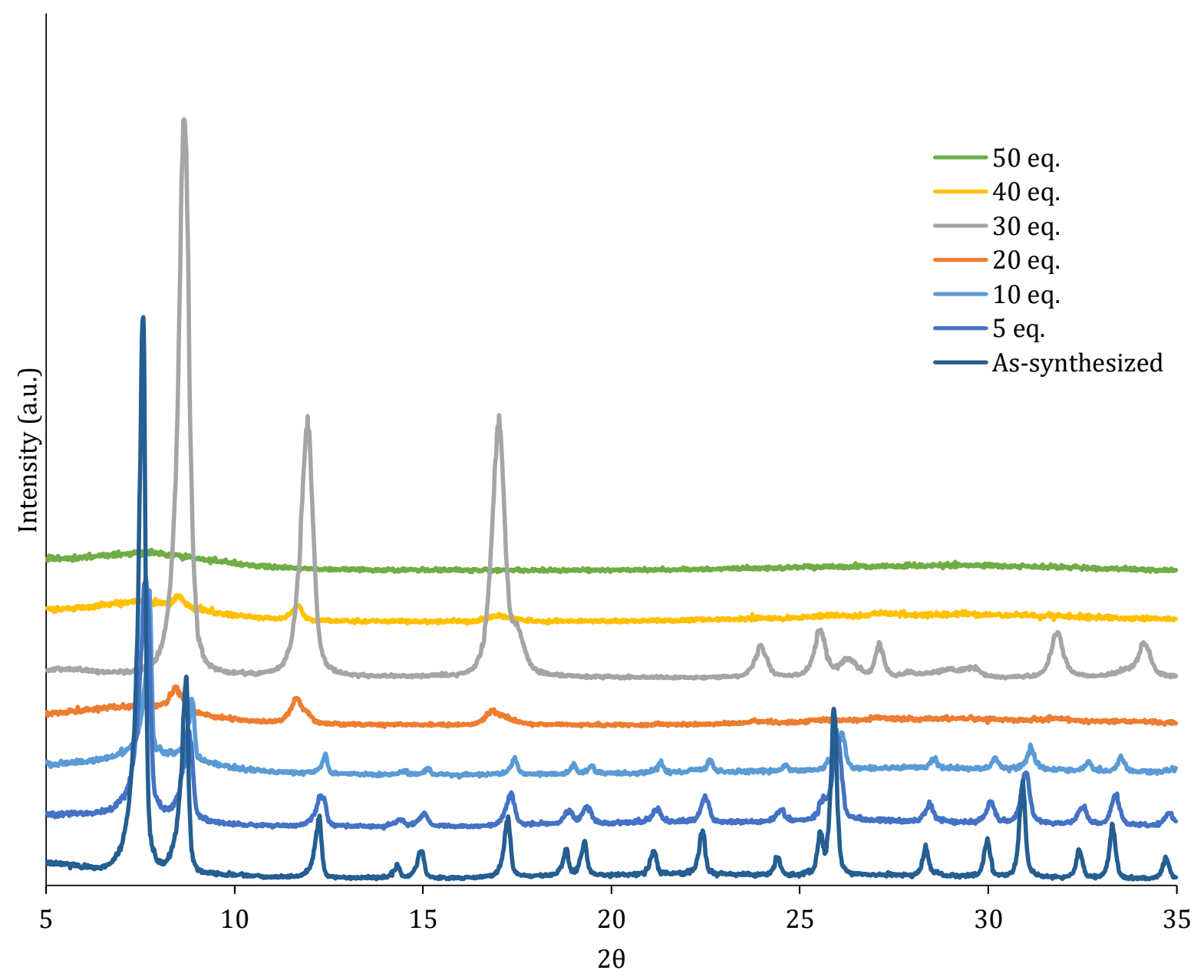

Figure 4.8.1.1 -1: XRD Pattern of samples produced varying EG concentrations.

The SEM images of the corresponding samples are presented in Figure 4.8.1.1-2. Firstly, it was noted that a 5 eq. of co-solvent EG produced a small number of octahedral crystals of approximately $0.5 \mu \mathrm{m}$ in size with majority of the crystallites being significantly smaller and irregular shaped of up to $0.05 \mu \mathrm{m}$ particle size. The sample mostly displayed a polydisperse crystals. On increasing the concentration to 10 eq., a large featureless smooth mass of material was observed. With further increase to 20 eq. EG, needle like fibres appear on the surface of the featureless block of material with complete transformation into the monodisperse needle-like fibrous structures at 30 eq. On increasing the EG quantity to 40eq., a stunted growth is observed in rod like structures sticking out of larger dough-like chunks of some amorphous material. Lastly, with 50 eq. EG, the fibers disappeared leaving behind a 
massive chuck of material with curvy rippled surface signalling negligible crystallinity of the material.

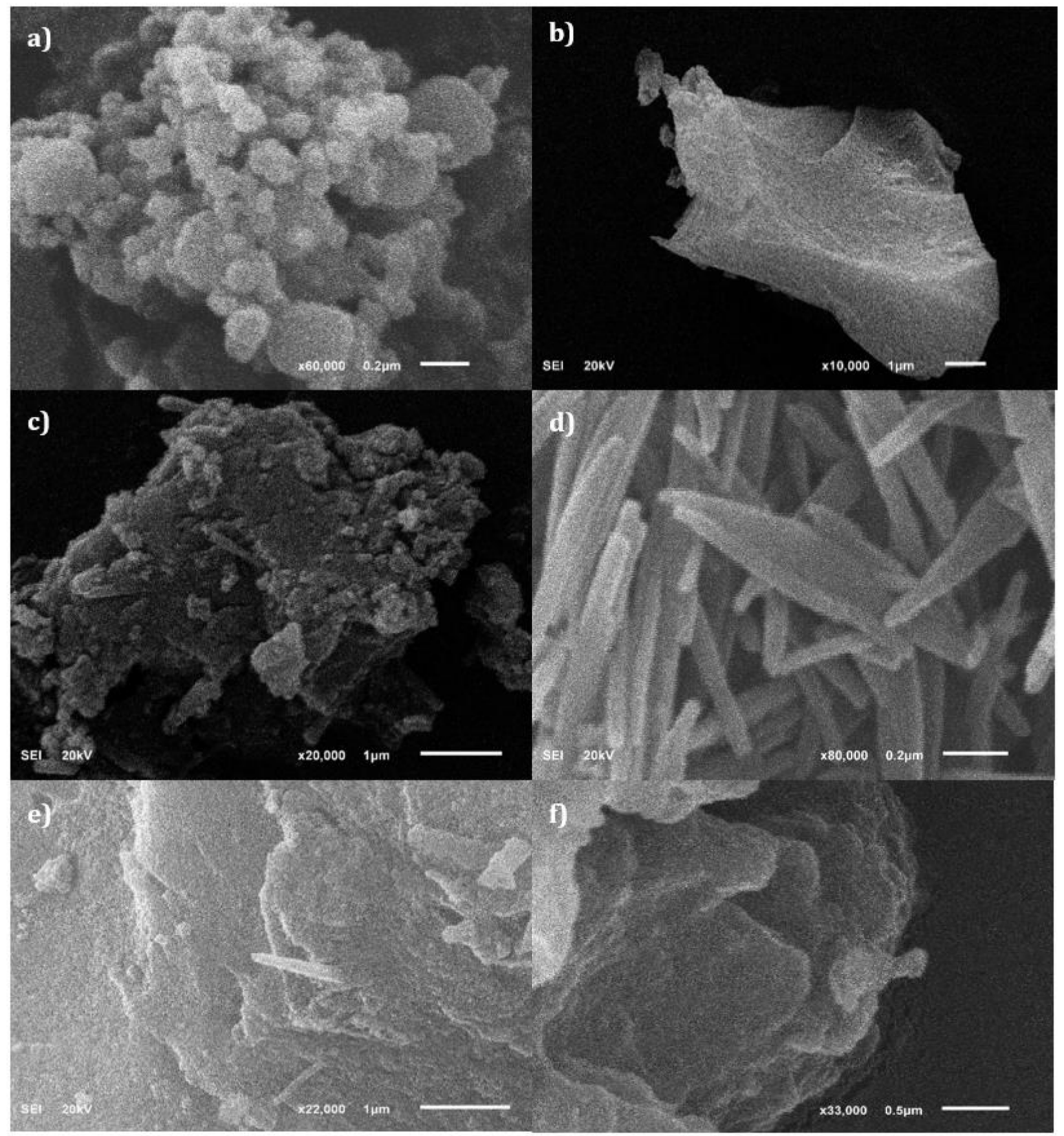

Figure 4.8.1.1-2: SEM images of samples produced with EG of a) 5eq. b) 10eq., c) 20eq., d) 30 eq. e) 40 eq., and e) 50 eq. 
The XRD and SEM results are in excellent agreement with the observations for all the samples. From the XRD data, it was identified that the rod-like structures had appeared only in the presence and with increasing quantity of EG. The reduction in crystallinity with the 5 and 10 eq. EG revealed that the smallest quantity of EG was adequate to suppress the growth of octahedral crystals leading to polydisperse crystals. From 20 eq. EG onwards, the morphology of UiO-66 crystals started to transform from octahedral to needle-like fibers where 30 eq. EG was identified as the critical co-solvent EG concentration, as increasing the EG concentration beyond 30 eq. resulted in amorphous material.

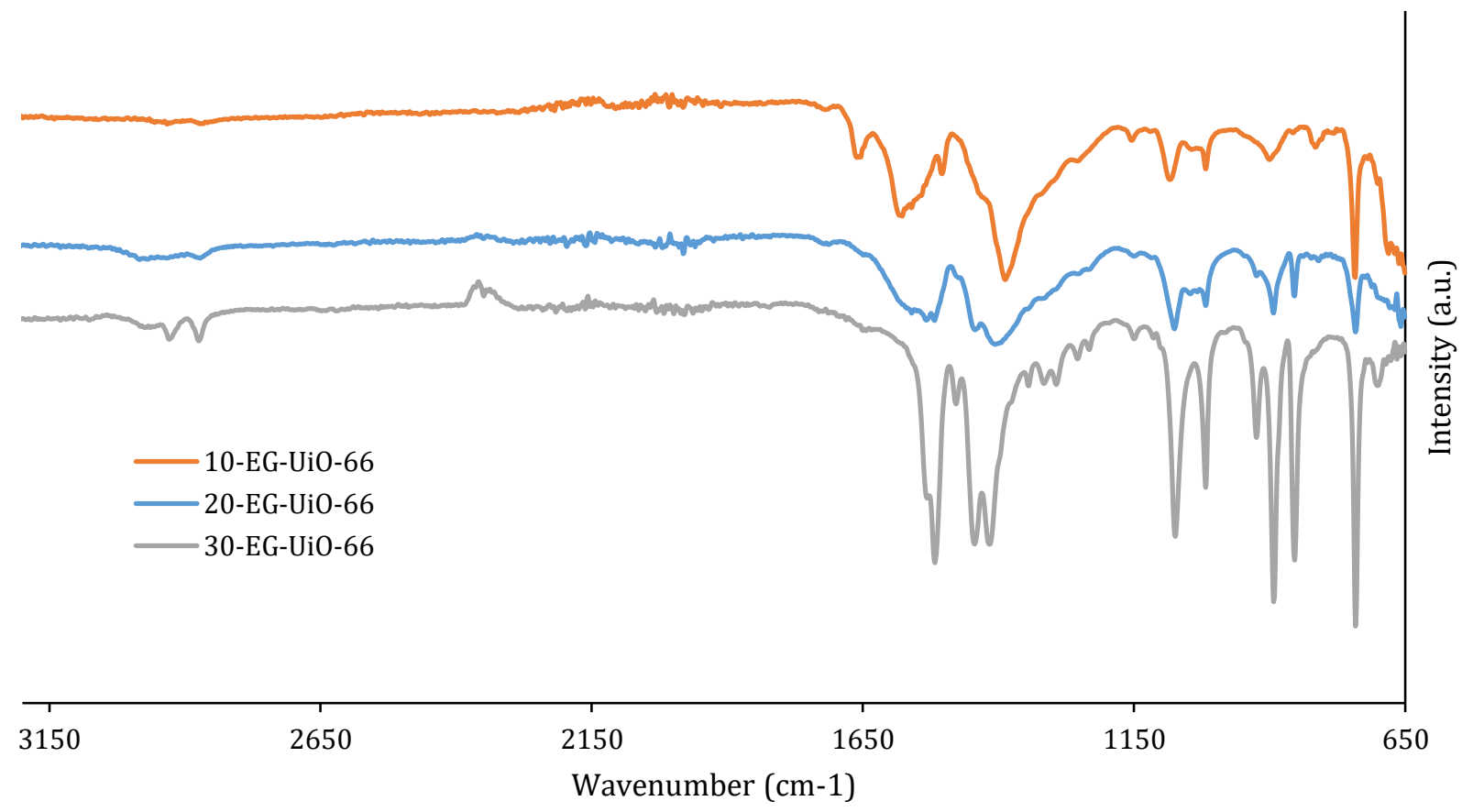

Figure 4.8.1.1-3: FTIR spectra of UiO-66 synthesized with different concentrations of EG as co-solvent.

A summary of the effect of 10 eq., 20 eq., and 30 eq. EG on the vibrational characteristics of various molecules of synthesized UiO-66 samples is depicted in Figure 4.8.1.1-3 and a comparison between their vibrational modes $\left(\mathrm{cm}^{-1}\right)$ is presented in Table 4.8.1.1-2. 
Table 4.8.1.1-2: Vibrational frequencies for UiO-66 samples synthesized with 10 eq., 20eq., and 30 eq. EG.

\begin{tabular}{|c|c|c|c|c|}
\hline $\begin{array}{c}\text { As- } \\
\text { synthesized } \\
\text { UiO-66 } \\
\end{array}$ & $\begin{array}{c}\text { 10-EG-UiO- } \\
66\end{array}$ & $\begin{array}{c}\text { 20-DEG-UiO- } \\
66\end{array}$ & $\begin{array}{c}\text { 30-EG-UiO- } \\
66\end{array}$ & Description \\
\hline- & - & - & 2926,2873 & BHET (OH group) [61] \\
\hline 1665.38 & 1660 & - & - & DMF (amide group) [41] \\
\hline 1589.9 & 1577.4 & 1557.6-1515 & - & $\begin{array}{c}\text { BDC (O-C-O asymmetric } \\
\text { stretching) [122] }\end{array}$ \\
\hline 1505.04 & 1503.68 & - & 1517.4 & $\begin{array}{c}\mathrm{BDC}(\mathrm{C}=\mathrm{C} \text { of benzene } \\
\text { rings })[122]\end{array}$ \\
\hline- & - & 1441 & 1460 & $\begin{array}{c}\text { BHET ( } \mathrm{CH}_{2} \text { bending) } \\
{[124]}\end{array}$ \\
\hline- & - & 1420 & $\begin{array}{l}1429.28 \\
1416.59\end{array}$ & $\begin{array}{c}\text { BHET (terminal OH } \\
\text { group of carboxylic acid) } \\
{[61]}\end{array}$ \\
\hline 1391.93 & 1388.7 & - & - & $\begin{array}{l}\text { O-C-O symmetric } \\
\text { stretching [122] }\end{array}$ \\
\hline 1155.03 & 1153.54 & - & 1148.36 & $\begin{array}{c}\mathrm{DMF}\left(\rho \mathrm{C}^{\prime} \mathrm{H}_{3}+\rho \mathrm{C}^{\prime \prime} \mathrm{H}_{3}\right) \\
{[125]}\end{array}$ \\
\hline 1091.81 & 1080.9 & - & - & $\operatorname{DMF}\left(\rho \mathrm{C}^{\prime} \mathrm{H}_{3}+v \mathrm{CN}\right)[125]$ \\
\hline- & - & 1075.89 & 1071.21 & $\begin{array}{c}\text { BHET (C=0 stretching) } \\
{[124]}\end{array}$ \\
\hline 1019.19 & 1017.88 & 1017.23 & 1017.86 & BDC $[122]$ \\
\hline- & - & - & 924.26 & $\mathrm{NH}_{3}[126]$ \\
\hline- & 898.9 & 895.34 & 892.3 & $\begin{array}{c}\text { BHET or EG }\left(\mathrm{CH}_{2}\right. \\
\text { rocking) }[124]\end{array}$ \\
\hline- & - & 853.19 & 853.81 & $\begin{array}{c}\text { BHET (vC-C stretching) } \\
{[61]}\end{array}$ \\
\hline 818.31 & 814.64 & - & - & $\begin{array}{c}\mathrm{OH} \text { bending }+\mathrm{CH} \\
\text { bending (anti-phase) } \\
{[41]}\end{array}$ \\
\hline 743.12 & 742.78 & 742.05 & 741 & $\begin{array}{l}\text { Zr-carboxylate group } \\
\text { bond [123] }\end{array}$ \\
\hline 670.1 & - & - & - & $\mu_{3}-0$ stretching $[41]$ \\
\hline
\end{tabular}

The IR spectra of the EG mediated UiO-66 samples were sequentially compared to the assynthesized sample's vibrational frequencies. Comparing the IR spectrum of 10 eq. EG 
sample with the as-synthesized UiO-66, apart from the regular frequencies discussed in Table 4.8.1.1-1, the two significant peaks were the appearance of peak $898.9 \mathrm{~cm}^{-1}$ and disappearance of $670.1 \mathrm{~cm}^{-1}$ which implied that the $\mu_{3}-0$ bridge formation was suppressed even with as low as 10 eq. quantity of EG. Nevertheless, the presence of all other bands in comparison to the as-synthesized sample was a good indicator of the integral part of UiO-66 still being retained within the sample. Upon increasing the EG quantity to 20 eq., a broad peak appeared between 1557.6-1515 cm-1 which was the transformation of peak $1584.6 \mathrm{~cm}^{-}$ 1 of 10 eq. EG-UiO-66 sample. The shift of the vibrational frequency to the lower wavenumber indicated an increased energy of the $\mathrm{O}-\mathrm{C}-\mathrm{O}$ bond, which eventually disappeared in the $30 \mathrm{eq}$. EG sample. This disappearance was in conjunction with the missing $\mu_{3}-0$ stretching confirmed the fact that this bond was absent in this sample. The prominent effect of 20 eq. EG was further marked by the appearance of $1441 \mathrm{~cm}^{-1}$ and $1420 \mathrm{~cm}^{-1}$ wavenumbers, which were related to the 30-EG-UiO-66 sample for the presence of BHET. The transition of these wavenumbers to a higher frequency in the transformed UiO-66 indicated the strengthening of the bonds between $\mathrm{CH}_{2}$ and terminal $\mathrm{OH}$ groups of carboxylic acid. It was highlighted that the wavenumbers related to BDC linkers (both symmetric and asymmetric stretching at $1388.7 \mathrm{~cm}^{-1}$ and $1557.6-1515 \mathrm{~cm}^{-1}$ ) along with missing $\mu_{3}-0$ stretching at $670.1 \mathrm{~cm}^{-1}$ strengthens the fact that the linkages between the zirconium clusters had changed to a different linkage with the increasing EG composition. Furthermore, the appearance of $1075.89 \mathrm{~cm}^{-1}$ solidified the existence of weak interactions of $\mathrm{C}=0$ bond of BHET within the sample. As it was justified in Section 4.7 that the new in-situ linker was BHET, the final similarities between the 30 eq. and 20 eq. EG synthesized UiO-66 samples were the peak presence at $895.34 \mathrm{~cm}^{-1}$ and $853.19 \mathrm{~cm}^{-1}$, both indicating the manifestation of the monomer BHET in the sample.

The transition of the structural formation of UiO-66 from octahedral crystals to needle shaped fibers was apparent from the changes in the FTIR spectra of the samples with the gradual increase in EG content from 10eq. to 30 eq. The FTIR implied significant changes in the bond strength and formation of different molecules with the changing quantity of EG in the samples. The disappearance of peak at $670.1 \mathrm{~cm}^{-1}$ and the transformation of $1584.6 \mathrm{~cm}^{-}$ 1 , both indicated that the bridging linkage between the metal clusters had been hampered 
with the presence of EG. The 10 eq. EG was sufficient to induce a minute structural transformation in the traditional UiO-66 crystals which was also confirmed by the XRD and SEM showing a non-uniform sample of octahedral and ill-defined crystals. Increasing the quantity to 20 eq. EG further strengthened the newly formed bonds and destabilized the $\mu_{3}-$ $\mathrm{O}$ bridging with complete disappearance of $\mathrm{O}-\mathrm{C}-\mathrm{O}$ bond. The EG composition caused appropriate interactions between the EG and BDC that promoted the formation of in-situ ligand BHET. This transitional stage was marked by the appearance of a smooth mass of material with minimal crystallinity in the SEM. The (200) and (220) peaks present in the 30 eq. EG sample also showed up in the 20 eq. EG sample.

\subsubsection{Conclusion}

The crystalline phase of Ui0-66 was heavily dependent upon the quantity of the ethylene glycol. There was a stark contrast in the results observed in both the crystallinity and morphology of new UiO-66 through XRD, SEM and FTIR. The characterization of all samples produced coherent results. It was concluded that 5 eq. EG was sufficient to bring about minute structural changes in the morphology of UiO-66. A 10 eq. concentration of EG significantly inhibited the octahedral growth of UiO-66 causing polydispersity of the sample. With increase of EG to 20 eq. and 30 eq., the structure leaned towards fibrous formation than traditional octahedral crystals; while surplus amount of EG, i.e. 40 eq. and 50 eq., damaged both the crystallinity and morphology of the fibrous material. With increasing concentration of EG, the XRD, SEM and FTIR revealed gradual changes in the morphology through intensified interactions between the EG and BDC linker molecules which resulted in an insitu formation of ligand monomer BHET suggesting that the newly synthesized ligand dictated the growth of UiO-66 crystals in (200). Nevertheless, more characterization techniques are required to determine the framework structure, the constituting elements present and the coordination modes of the ligands in order to confirm the results. However, the results coherently agree that a 30 eq. concentration of EG was necessary for the anisotropic growth of UiO-66 crystals. 


\subsubsection{DIETHYLENE GLYCOL}

\subsubsection{Results and Discussion}

From Section 4.7 it was established that 30 eq. concentration of co-solvent DEG successfully promoted the extension of Ui0-66 framework resulting in large octahedral crystals. As solvothermal syntheses are sensitive to the reaction conditions, specially the reactant composition, the motive of this experiment was to follow up the effect of DEG concentration on UiO-66 crystals using Scheme 3 at 10 eq., 20 eq., and 30 eq. DEG by stirring for 24h with subsequent heating at $120^{\circ} \mathrm{C}$ for $96 \mathrm{~h}$. The reactant concentrations and reaction conditions are summarized in Table 4.8.2.1-1. The as-synthesized sample was generated without the co-solvent with a stir time of $24 \mathrm{~h}$ which was subsequently heated at $120^{\circ} \mathrm{C}$ for $24 \mathrm{~h}$ and is bolded for reference.

Table 4.8.2.1-1: Reactant composition and reaction conditions to investigate the effect of DEG concentration.

\begin{tabular}{cccccccc}
\hline $\begin{array}{c}\mathrm{ZrCl}_{4} \\
(\mathbf{m m o l})\end{array}$ & $\begin{array}{c}\mathbf{1 , 4 - B D C} \\
(\mathbf{m m o l})\end{array}$ & $\begin{array}{c}\text { DMF } \\
(\mathbf{e q} .)\end{array}$ & $\begin{array}{c}\text { Acetic } \\
\text { Acid } \\
\text { (eq.) }\end{array}$ & $\begin{array}{c}\text { Glycol } \\
\mathbf{E q} .\end{array}$ & $\begin{array}{c}\text { Stirring } \\
\text { time } \\
\text { (h) }\end{array}$ & $\begin{array}{c}\text { Temperature } \\
\left({ }^{\circ} \mathbf{C}\right)\end{array}$ & $\begin{array}{c}\text { Reaction } \\
\text { time (h) }\end{array}$ \\
\hline $\mathbf{0 . 3 4 3}$ & $\mathbf{0 . 3 4 3}$ & $\mathbf{8 6 6}$ & $\mathbf{1 6 0}$ & $\mathbf{0}$ & $\mathbf{2 4}$ & $\mathbf{1 2 0}$ & $\mathbf{2 4}$ \\
0.343 & 0.343 & 866 & 160 & 10 & 24 & 120 & 96 \\
0.343 & 0.343 & 866 & 160 & 20 & 24 & 120 & 96 \\
0.343 & 0.343 & 866 & 160 & 30 & 24 & 120 & 96 \\
\hline
\end{tabular}

The XRD and SEM results are shown in Figure 4.8.2.1-1 and Figure 4.8.2.1-2, respectively. As seen in Figure 4.8.2.1-1, the diffractograms matched perfectly with the literature suggesting pure phase UiO-66 crystals. The peak intensities were found to be subordinate for both 10 and 20 eq. DEG concentrations and remained the same for the entire $2 \theta$ range in comparison to the as-synthesized pattern. The lower intensities and peak broadening indicated a 
deterioration in crystallinity. However, it was noted that 10 eq. was better than 20 eq. DEG in terms of the intensity of diffraction peaks. Increasing the amount to 30 eq. DEG produced narrower peaks with higher intensity compared to the as-synthesized sample for all $2 \theta$ angles. The higher intensities reflected enhanced growth and higher crystallinity for 30 eq. DEG and diminished in crystallinity for 10 and 20 eq. DEG samples.

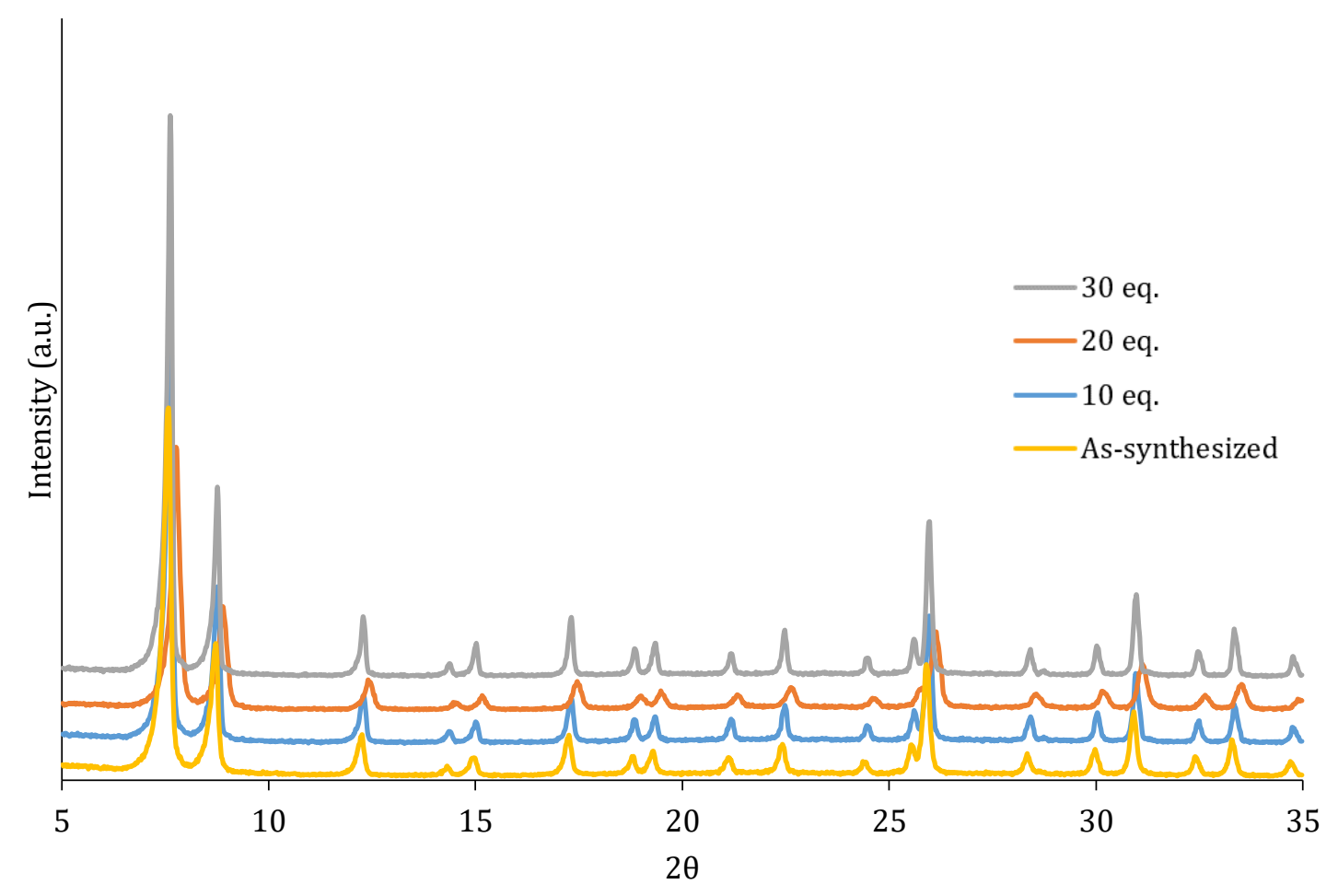

Figure 4.8.2.1-1: XRD patterns for UiO-66 samples with varying DEG concentrations.

The SEM images in Figure 4.8.2.1-2 also reflected similar conclusions. A 10eq. of DEG produced both polydisperse sharp and round edged crystals of $0.45 \mu \mathrm{m}$ with a significant number showing surface defects and/or twining. The 20 eq. DEG also showed similar results as the 10 eq. samples with reduced polydispersity and deformation characteristics with roughly $0.2 \mu \mathrm{m}$ in size. The crystals also appeared to be more agglomerated than the previous sample. However, the crystals failed to retain the octahedral shape consisting multiple forms. Lastly, the 30 eq. sample showed formation of narrowly dispersed round edged octahedral crystals with up to $1 \mu \mathrm{m}$ in size with some tumor like growth on the surface. The SEM also indicated narrow size distribution at higher concentration and vice versa. Additionally, it 
was observed that as the DEG content was increased in the solution, the particle size increased significantly from $0.2 \mu \mathrm{m}$ to $1 \mu \mathrm{m}$ in size.
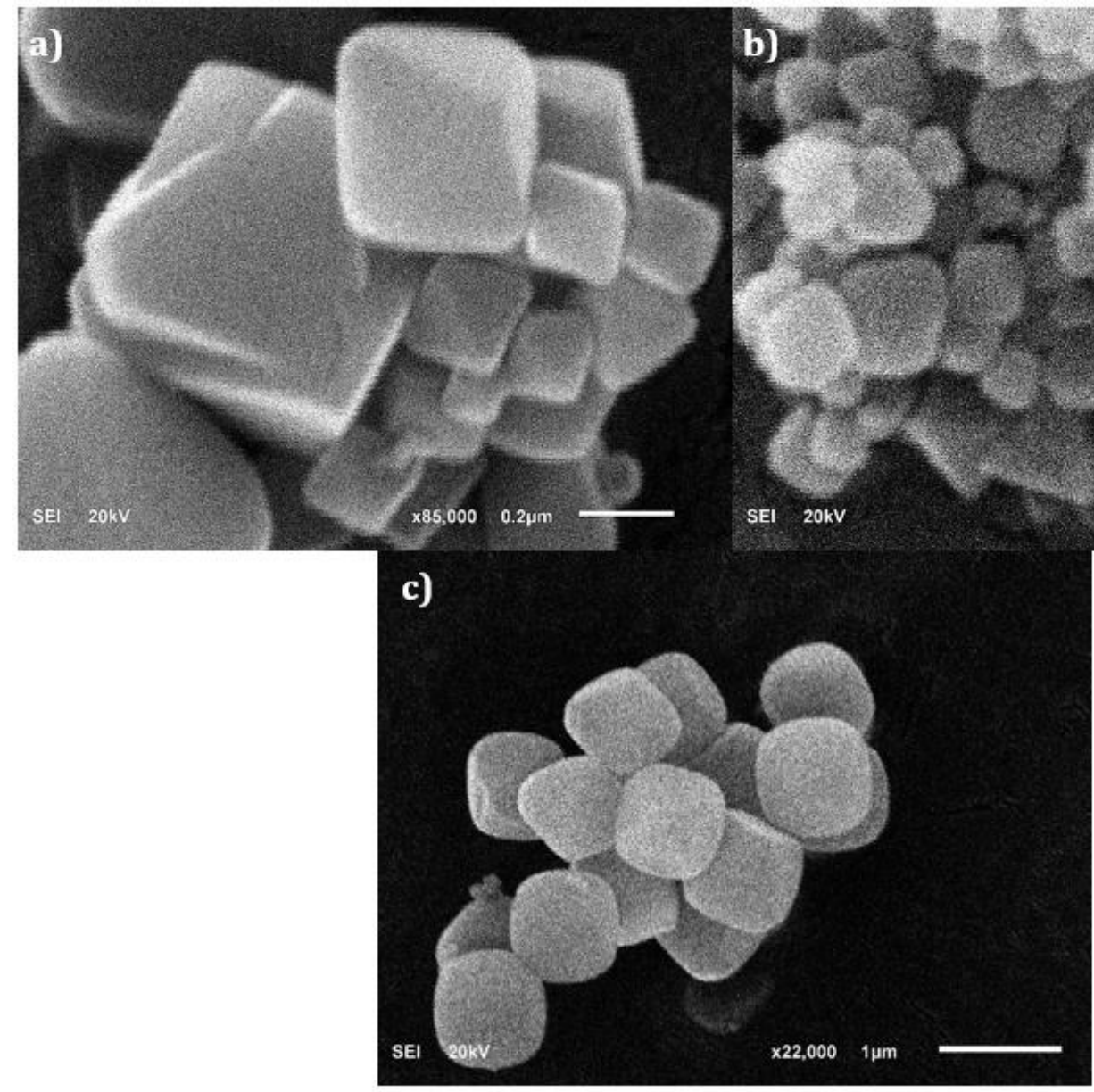
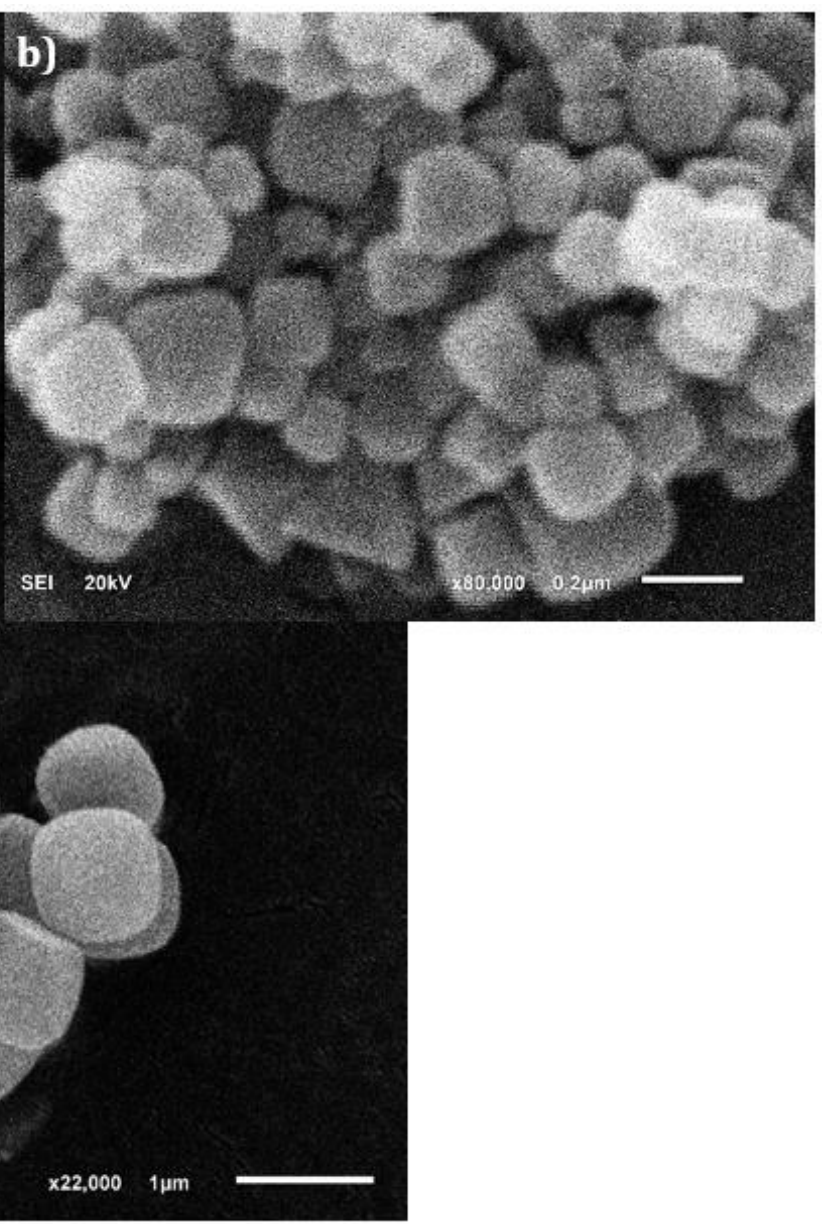

Figure 4.8.2.1-2: SEM images of samples produced with DEG of a) 10eq., b) 20eq., and c) 30 eq.

The crystallinities were calculated by the peak ratio method introduced in Section 4.2.2. The change in crystallinity of UiO-66 crystals with the introduction of DEG as a co-solvent is summarized in Figure 4.8.2.1-3. The non-uniformity in UiO-66 crystals in 10 eq. and 20 eq. DEG suggested a slow nucleation rate during its synthesis [107]. The crystal growth was regulated such that the production of primary nuclei slowed down the depletion of resources within the solution. This caused simultaneous generation of secondary nuclei resulting in various crystal sizes. It was noted that, increase in DEG composition showed a systematic increase in the homogeneity of the UiO-66 crystals. From the SEM images, at lower concentrations of DEG, the particles appeared more polydisperse and clustered in formation, 
while with use of higher DEG content, the particles become homogeneous and discrete. According to the literature, there were no reported interactions between DEG with other species in the reaction mixture. Thus, the occurrence was best explained by the assessment of $\mathrm{p} K_{\mathrm{a}}$ values.

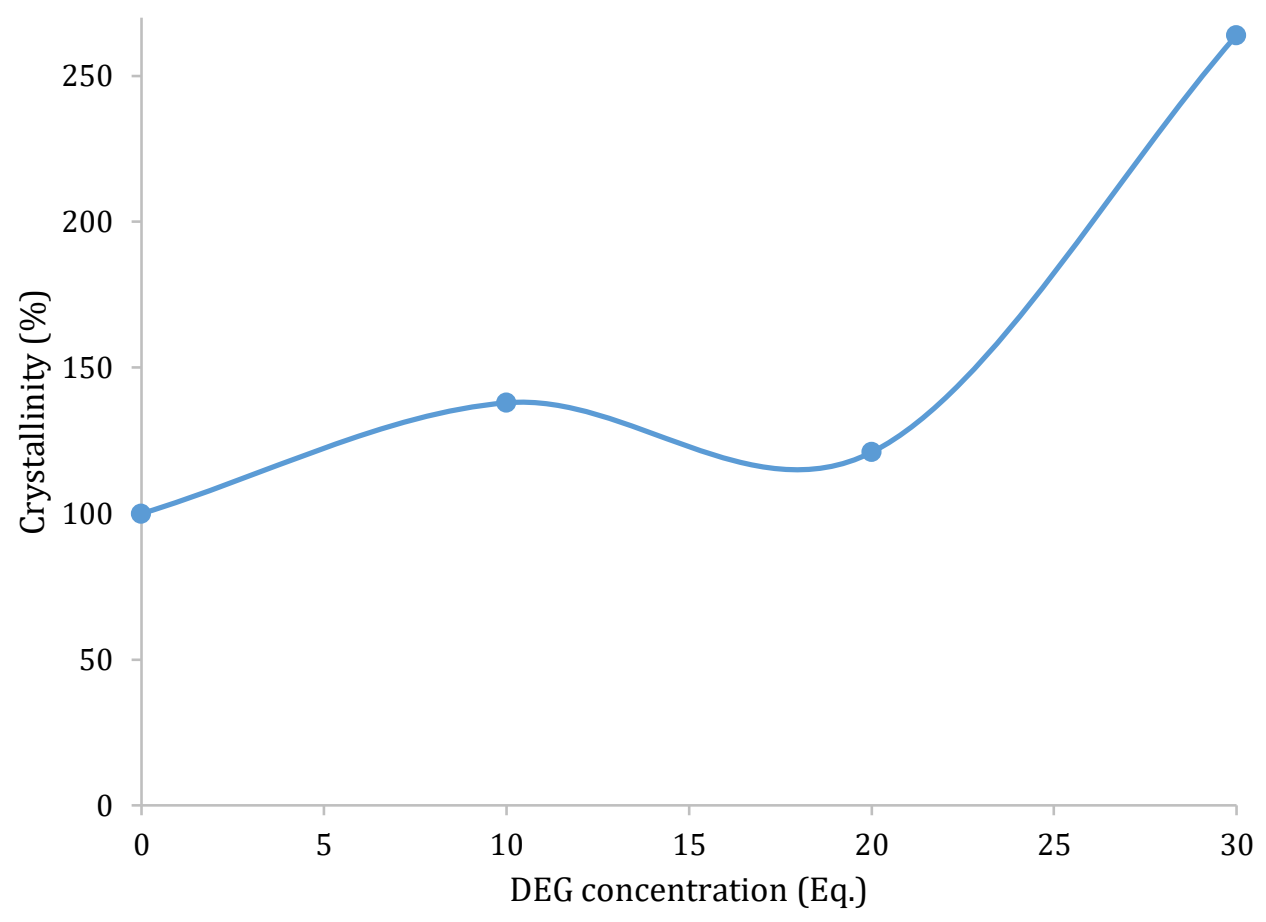

Figure 4.8.2.1-3: Effect of DEG concentration on the crystallinity of UiO-66 particles.

For the effect of DEG concentration experiments, DEG was added into the solution consecutively into the DMF before all other reactants. As explained in Section 4.7, the high $\mathrm{p} K_{\mathrm{a}}$ value of DEG (14.2) increased the basicity of the solution that caused the DMF to readily protonate in the solution. As a result, the use of higher DEG as a co-solvent of 30 eq. heightened the deprotonation od BDC and acetic acid molecules, along with the competitive reactions between them. As acetate molecules replaced some of the BDC linker molecules, they compensated for the charges of the missing linker increasing the number of both missing linkers and missing clusters that created larger pores than usual [112]. Thus, the integration of more acetate molecules in the framework produced large octahedral UiO-66 crystals [112]. The assumption of this dynamic is logical based on the fact that the strength of a metal-ligand bond increases as the basicity of the ligand increases [137], [138]. Thus, 
increasing the concentration of DEG led to larger octahedral UiO-66 crystals. However, this assumption requires further analysis of the resultant UiO-66 particles through elemental analysis and characterization techniques that will help determine the constituting molecules in the resultant UiO-66 framework.

\subsubsection{Conclusion}

In summary, both the XRD and the SEM drew similar conclusions in terms of crystallinity and structural properties of UiO-66. The increasing quantity of DEG in the solution increased the $\mathrm{pH}$ of the solution which resulted in more acetic acid molecules to be protonated within the solution which was incorporated within the Ui0-66 framework created more defects, hence larger particle size. Thus, the increased molecular concentration of DEG was indirectly associated with slowing down the nucleation rate that led to uniform growth of large UiO-66 crystals. Therefore, the crystallinity of UiO-66 was successfully enhanced following Scheme 3 with 30 eq. DEG.

\subsubsection{TRIETHYLENE GLYCOL}

\subsubsection{Results and Discussion}

Similar to the effect of co-solvent DEG concentration on the formulation of UiO-66 crystals, the effect of TEG was studied using Scheme 3 for 10, 20, and 30 eq. TEG also showed relative improvement in crystallinity of UiO-66. The results were compared for samples synthesized using Scheme 3 for 10 eq., 20 eq., and 30 eq. TEG concentrations by stirring for 24h with subsequent heating at $120^{\circ} \mathrm{C}$ for $96 \mathrm{~h}$. The reactant concentrations and reaction conditions are summarized in Table 4.8.3.1-1. The as-synthesized sample was generated without the co-solvent with a stir time of $24 \mathrm{~h}$ which was subsequently heated at $120^{\circ} \mathrm{C}$ for $24 \mathrm{~h}$ and is bolded for reference. 
Table 4.8.3.1-1: Reactant composition and reaction conditions to investigate the effect of TEG concentration.

\begin{tabular}{|c|c|c|c|c|c|c|c|}
\hline $\begin{array}{c}\mathrm{ZrCl}_{4} \\
(\mathbf{m m o l})\end{array}$ & $\begin{array}{l}\text { 1,4-BDC } \\
\text { (mmol) }\end{array}$ & $\begin{array}{l}\text { DMF } \\
\text { (eq.) }\end{array}$ & $\begin{array}{c}\text { Acetic } \\
\text { Acid } \\
\text { (eq.) }\end{array}$ & $\begin{array}{c}\text { Glycol } \\
\text { Eq. }\end{array}$ & $\begin{array}{c}\text { Stirring } \\
\text { time } \\
\text { (h) }\end{array}$ & $\begin{array}{c}\text { Temperature } \\
\left({ }^{\circ} \mathrm{C}\right)\end{array}$ & $\begin{array}{l}\text { Reaction } \\
\text { time (h) }\end{array}$ \\
\hline 0.343 & 0.343 & 866 & 160 & 0 & 24 & 120 & 24 \\
\hline 0.343 & 0.343 & 866 & 160 & 10 & 24 & 120 & 96 \\
\hline 0.343 & 0.343 & 866 & 160 & 20 & 24 & 120 & 96 \\
\hline 0.343 & 0.343 & 866 & 160 & 30 & 24 & 120 & 96 \\
\hline
\end{tabular}

From Figure 4.8.3.1-1, the diffraction patterns of UiO-66 samples containing TEG showed good match with the literature diffraction pattern. As shown in Figure 4.8.3.1-1, 5 eq. TEG instantly reduced the peak intensities but with negligible peak broadening in the diffractogram. The lowering of intensity indicated that the sample contains less crystals oriented in that direction. Upon increasing the TEG concentration to 10 eq., the diffraction pattern intensities aggravated for the entire $2 \theta$ range, which indicated increased degree of order of the UiO-66 crystals. However, further increase in TEG content, i.e. 20 eq., degraded the enhanced crystallinity which was indicated by peak broadening and reduction in peak intensity. Contrastingly, the addition of 30 eq. TEG then enhanced the crystallinity of the sample. The sample with 10 eq. TEG showed the highest crystallinity according to the XRD results. Thus, in terms of the peak intensities and area, the order of the increasing crystallinity for the co-solvent aided UiO-66 synthesis was 5 eq. $<20$ eq. $<30$ eq. $<10$ eq. 


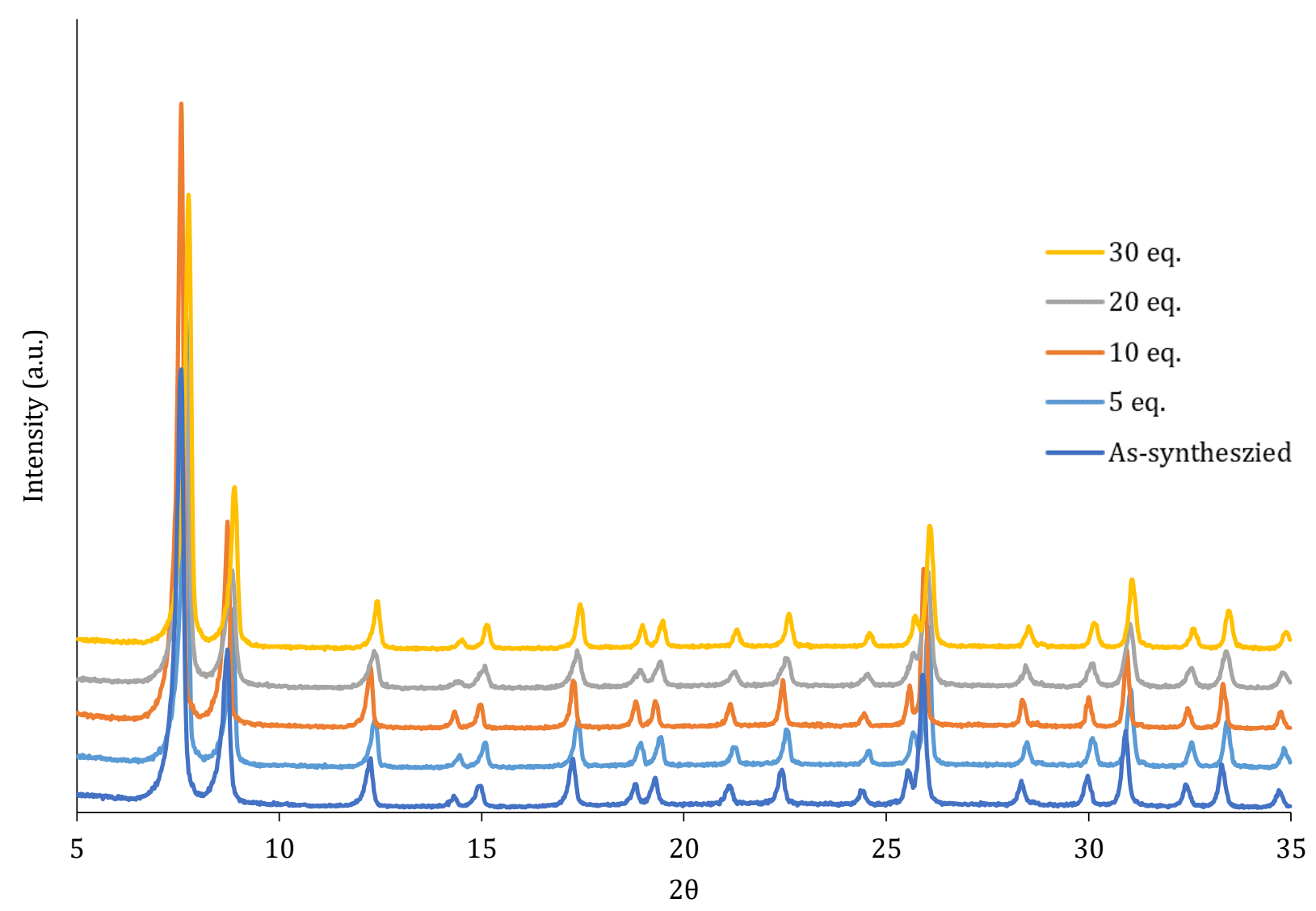

Figure 4.8.3.1-1: XRD patterns for UiO-66 samples with varying TEG concentrations.

The SEM images of the corresponding UiO-66 samples are represented in Figure 4.8.3.1-2. It was evident that the inclusion of TEG as a co-solvent in the solvothermal synthesis of UiO-66 introduced high degree of polydispersity in the samples. The SEM images for 5eq. TEG showed sharped edged polydisperse growth of octahedral crystal size ranging from 0.8-0.2 $\mu \mathrm{m}$ with some defective facets. A $10 \mathrm{eq}$. TEG, on the other hand, showed larger polydisperse crystals of about $1 \mu \mathrm{m}$ to $0.5 \mu \mathrm{m}$ in size with rounded UiO-66 crystals. The sample containing 5 eq., 10 eq., and 30 eq. TEG showed small tumor growth. Increasing the concentration of TEG to 20 eq. showed smaller deformed octahedral crystals of about $0.2 \mu \mathrm{m}$ in size, while 30 eq. produced polydisperse particles with $0.8-0.2 \mu \mathrm{m}$ in size with no visible outgrowth. In addition, the surface defect was also sustained in this sample. 


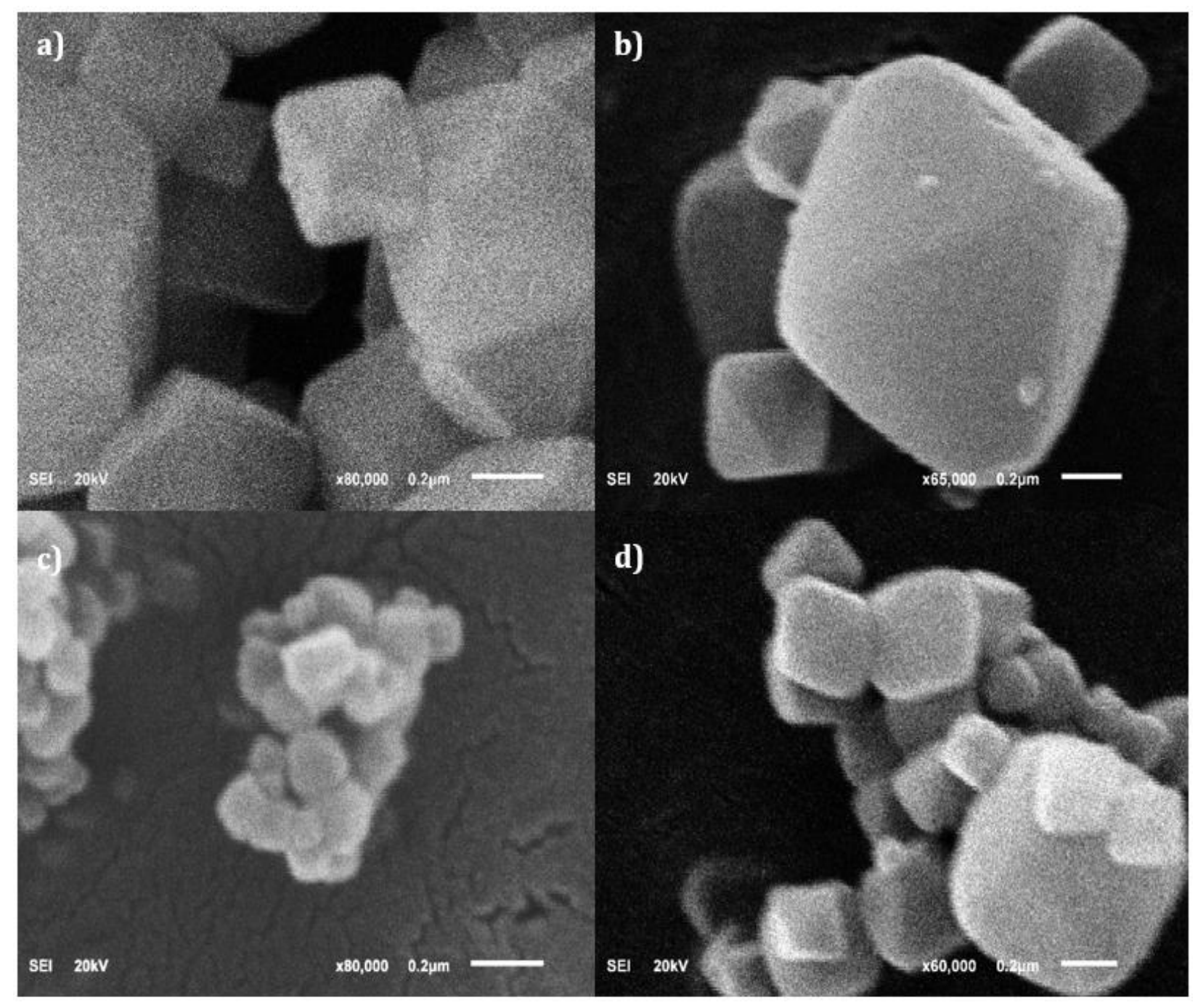

Figure 4.8.3.1-2: SEM images of samples produced with TEG of a) 5eq. b) 10eq., c) 20eq., d) 30 eq.

The crystallinities were calculated by the peak ratio method introduced in Section 4.2.2. The change in crystallinity of the UiO-66 crystals with the introduction of TEG as a co-solvent is represented in Figure 4.8.3.1-3. The crystallinity of the particles increased with the increase in concentration of TEG up to 10 eq. TEG and then dropped. Unlike the effect of co-solvent DEG, the incorporation of TEG as a co-solvent in the reaction mixture proved to be a disadvantage in the formation of UiO-66 crystals as it produced polydisperse crystals despite the crystallinity being higher. The surface defects and increased polydispersity of the particles were sustained at all concentration of TEG. 


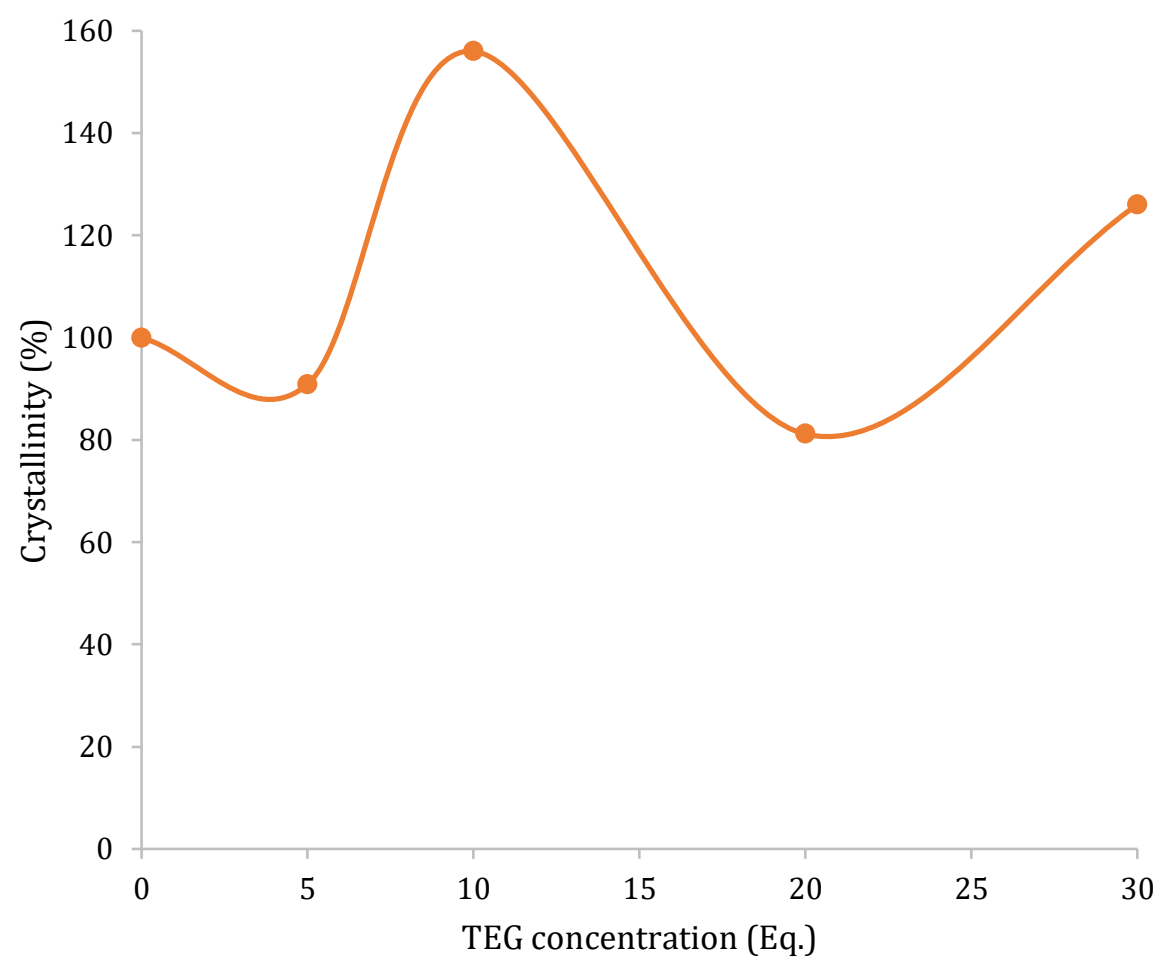

Figure 4.8.3.1-3: Effect of TEG concentration on the crystallinity of UiO-66 particles.

The addition of TEG in the precursor solution increased the basicity which led to the high competition of BDC and acetate molecules, and thus, larger crystallites. The only difference between the case of TEG and DEG was the increased bulky alkyl group. Thus, we predicted that although the higher TEG content was sufficient in increasing the particle size of the UiO66 crystals, the longer alkyl chain length hindered the successful incorporation of acetic acid into the SBU. As a result, the resultant UiO-66 crystals always remained polydisperse in nature. However, this assumption requires investigation of the resultant UiO-66 particles through elemental analysis and characterization techniques that will help determine the constituting molecules in the resultant UiO-66 framework.

The particle size indicated no general trend in either increasing or decreasing manner. At lower concentrations of TEG, the particles appear more discrete with fewer surface defects. The increasing molecular concentration of TEG in the solution, similar to DEG, enriched the mixture with deprotonated acetate molecules. As the acetate molecules had a better chance of bonding with the $\mathrm{Zr}$ atoms, justified by the $\mathrm{p} K_{\text {a }}$ values, the SBUs grew in that fashion incorporating more acetate instead of the carboxylate group of BDC, making the subsequent 
UiO-66 crystals larger in size. These observances were unanimously drawn from the XRD and SEM results. In this case, the contrast in the behavior from DEG may have risen due to the longer alkyl chain in the molecules. Thus, instead of introducing competitive reactions between the acetic acid molecules and BDC linkers, the conjunction of acetic acids and $\mathrm{Zr}$ atoms hindered, resulting in large polydisperse crystals with a broader particle size distribution.

\subsubsection{Conclusion}

The aim of this experiment was to assess the effect of TEG on the morphology of UiO-66 by increasing the TEG content in the solution from 5eq. to 30eq. The results were similar to the ones obtained in the DEG effect on the morphology of UiO-66. The XRD and the SEM indicated large polydispersity in the UiO-66 crystals. The increasing quantity of TEG in the reaction mixture, due to the lengthy alkyl chain, caused non-uniform growth of crystals. Thus, it can be said that TEG improved the crystal size following Scheme 3 with 30 eq. TEG but compromised the homogeneity and defect free surface of UiO-66 crystals.

\subsection{EFFECT OF MODULATOR ON EG AIDED UiO-66}

\subsubsection{Results and Discussion}

The importance of modulator on the solvothermal synthesis was demonstrated in Section 4.6. The strong hydrophilic character of $\mathrm{Zr}$ makes it almost impossible to synthesize high quality crystals without the use of a modulator. According to literature review, when a modulator is absent in the solution mixture, the growth of crystals proceeds in an unregulated manner from multiple primary nuclei. The uncontrolled growth thus leads to formation of small agglomerated particles with poor crystallinity. According to this concept, the same rule should follow for the UiO-66 crystals synthesized via Scheme 3 with 30 eq. cosolvent EG. 
Thus, to evaluate whether acetic acid was necessary to produce these unique morphologies, experiments were performed using four different variations. UiO-66 crystals were synthesized (1) with acetic acid and 30 eq. EG at $120^{\circ} \mathrm{C}$ for $96 \mathrm{~h},(2)$ without acetic acid and 30 eq. EG sample synthesized at $120^{\circ} \mathrm{C}$ for $96 \mathrm{~h}$, (3) without EG or acetic acid and without acetic acid at $120^{\circ} \mathrm{C}$ for $24 \mathrm{~h}$, and (4) with acetic acid and no EG at $120^{\circ} \mathrm{C}$ for $24 \mathrm{~h}$. All samples were synthesized via Scheme 3 with a stirring time for $24 \mathrm{~h}$. The as-synthesized sample was no. (4). The reactant concentrations and reaction conditions are summarized in Table 4.9.11. The as-synthesized sample was generated without the co-solvent with a stir time of $24 \mathrm{~h}$ which was subsequently heated at $120^{\circ} \mathrm{C}$ for $24 \mathrm{~h}$ and is bolded for reference.

Table 4.9.1-1: Reactant composition and reaction conditions to investigate the role of acetic acid in EG synthesized UiO-66 crystals.

\begin{tabular}{cccccccc}
\hline $\begin{array}{c}\mathrm{ZrCl}_{4} \\
(\mathbf{m m o l})\end{array}$ & $\begin{array}{c}\text { 1,4-BDC } \\
\text { (mmol) }\end{array}$ & $\begin{array}{c}\text { DMF } \\
\text { (eq.) }\end{array}$ & $\begin{array}{c}\text { Acetic } \\
\text { Acid } \\
\text { (eq.) }\end{array}$ & EG (eq.) & $\begin{array}{c}\text { Stirring } \\
\text { time (h) }\end{array}$ & Temp ( $)$ & $\begin{array}{c}\text { Time in } \\
\text { oven (h) }\end{array}$ \\
\hline 0.343 & 0.343 & 866 & 30 & 30 & 24 & 120 & 96 \\
$\mathbf{0 . 3 4 3}$ & $\mathbf{0 . 3 4 3}$ & $\mathbf{8 6 6}$ & $\mathbf{3 0}$ & $\boldsymbol{0}$ & $\mathbf{2 4}$ & $\mathbf{1 2 0}$ & $\mathbf{2 4}$ \\
0.343 & 0.343 & 866 & 0 & 30 & 24 & 120 & 96 \\
0.343 & 0.343 & 866 & 0 & 0 & 24 & 120 & 96 \\
\hline
\end{tabular}

The effect of acetic acid as a modulator was clear on comparing the XRD patterns for all the 4 samples with the as-synthesized sample (Figure 4.9.1-1). Firstly, a comparison between (3) and (4) UiO-66 crystals showed a significant difference in the diffractograms. The assynthesized sample pattern was in good agreement with the literature values (Figure 4.1.22). The sample synthesized without acetic acid showed noteworthy decline in peak intensity and increase in peak broadening. This indicated decline in crystal order as well as poor formulation of crystals. This means that although UiO-66 can be synthesized without acetic acid, the inclusion of acetic acid in the precursor solution accelerates the production of UiO66 crystals, requiring shorter crystallization time. Secondly, a comparison of the non- 
modulated and modulated samples in the presence of EG showed expected outcomes. The sample produced with EG and acetic acid resulted in peak shifting to the higher angle, along with heightened intensities at (200) direction indicating oriented growth of UiO-66 crystals. Besides, the diffractogram revealed complete suppression of peak at (111) direction. On the other hand, the sample without acetic acid EG aided synthesis yielded a humped diffractogram with no characteristic peaks. This showed that the sample was amorphous with presence of only EG co-solvent in the mixture and that acetic acid was necessary to yield the supposedly unique UiO-66 framework.

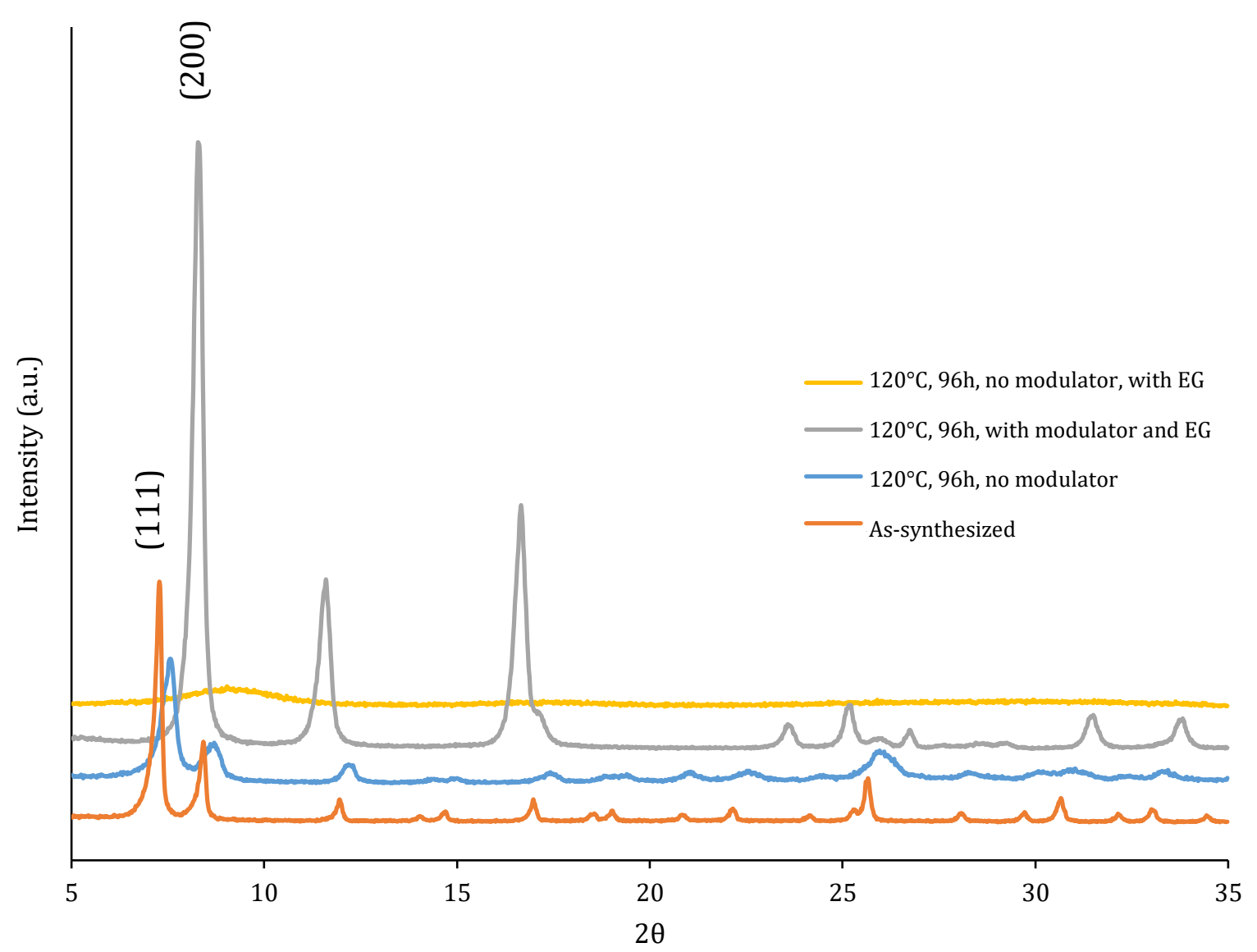

Figure 4.9.1-1: XRD patterns illustrating the effect of modulator on the morphology of assynthesized and EG aided UiO-66 crystals.

The SEM images, as depicted in Figure 4.9.1-2, also demonstrated the expected results from the XRD patterns. From the synthesized UiO-66 samples without co-solvent, the samples 
devoid of acetic acid were ill-defined and heavily agglomerated with $<0.05 \mu \mathrm{m}$ in size; while in the presence of acetic acid, the particles were defined and faceted structures. Contrariwise, the absence of acetic acid in EG aided UiO-66 synthesis, i.e. (1), demonstrated clustered growth of size between 0.1-0.5 $\mu \mathrm{m}$. However, in this case EG increased the particle size from $<0.05 \mu \mathrm{m}$ to $\sim 0.5 \mu \mathrm{m}$. With acetic acid, however, the UiO-66 crystals converted from amorphous to highly crystalline needle-like crystals.

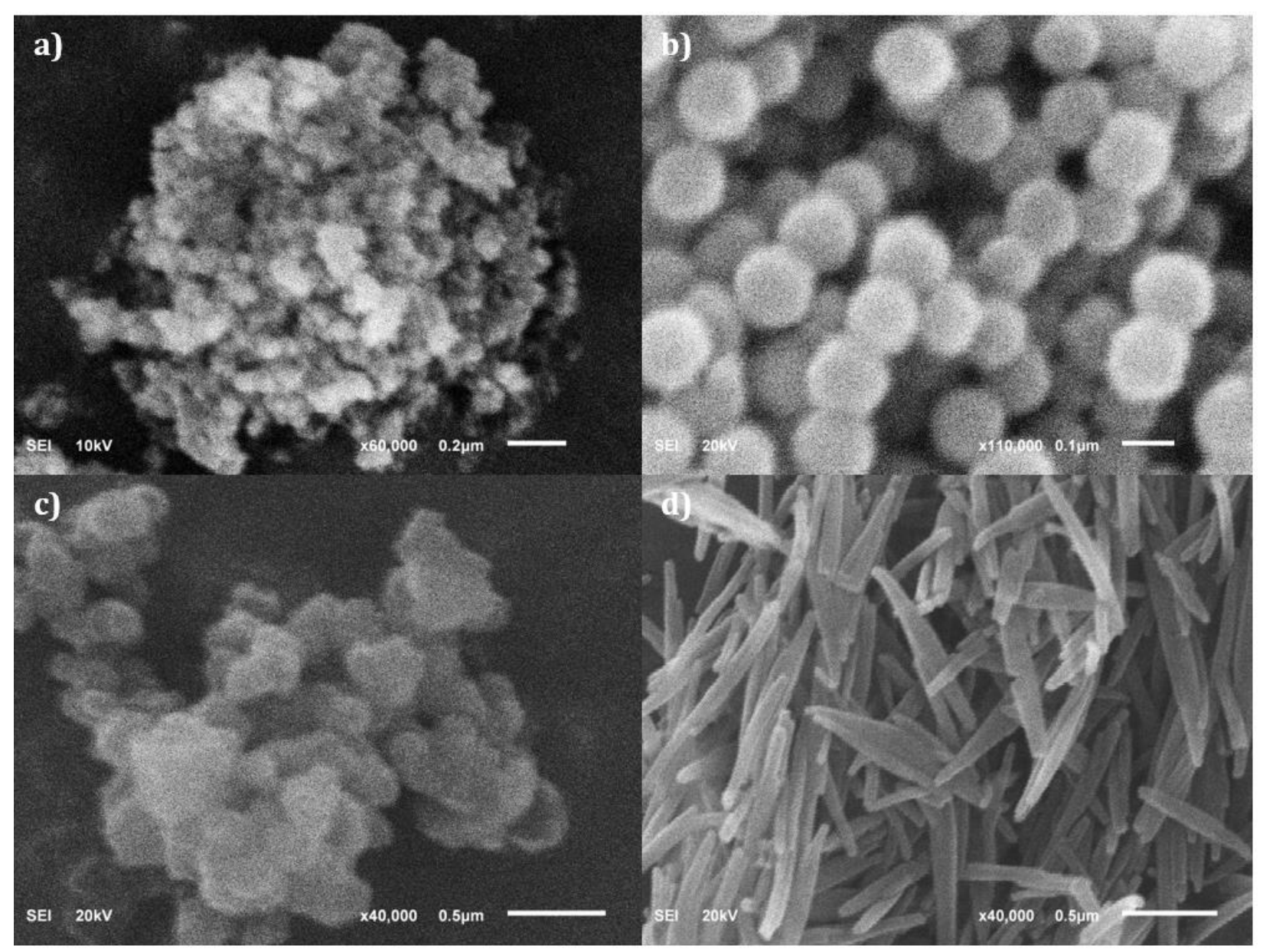

Figure 4.9.1-2: SEM images of UiO-66 samples: a) without EG and acetic acid, b) without EG with acetic acid, c) with EG without acetic acid, and d) with EG and with acetic acid.

Evidently, the absence of acetic acid produced ill-defined clustered morphologies, while acetic acid regulated the morphologies to be more well-defined and highly crystalline materials. The observations are absolutely consistent with the literature review [38], [68], 
[87], [109]. In the case of UiO-66 particles without EG, acetic acid as a modulator, effectively altered the thermodynamic equilibrium of the reaction by effectively competing with the linker molecules in order to occupy the sites of attachment of BDC and limited the number of nucleation sites. In process of this growth, the acetic acid molecules exchanged with the linker molecules in the transitional SBUs. This mechanism prevented the aggregation of molecules, allowing the particles to grow larger in size. These new SBUs formed through an exchange reaction between ligands and themselves become the transitional complexes in building the product [4]. Thus, increase in the modulator concentration impedes the binding of linkers to the metal center [4] limiting the growth and number of nucleation sites, and thereby, enhancing the growth of discrete crystals [109]. This competition between the acetic acid and the BDC molecules to bind to the central metal $\mathrm{Zr}$ leads to the production of large octahedral crystals. Moreover, this observation is consistent with the results produced in Section 4.6, which was the effect of excess modulator content.

In the case of EG aided synthesis of UiO-66 crystals, the absence of acetic acid produced similar effect of uncontrolled growth. On the other hand, in the presence of EG, due to the high $\mathrm{p} K_{\mathrm{a}}$ value of $\mathrm{EG}$ (14.22) the basicity/pH of the solution also increased. The higher value of $\mathrm{pH}\left(>\mathrm{p} K_{\mathrm{a}}\right.$ of DMF (6.7)) caused the DMF to readily protonate in the solution which deprotonated the acetic acid in amounts more than the conventional DMF solution. Thus, the proton rich solution facilitated the reaction between the BDC linkers and EG producing BHET monomer which was proved in Section 4.7. Consequently, the presence of acetic acid in the solution was logically necessary in order to produce BHET. The likelihood of this occurrence was also evident from the sample in which acetic acid was absent. In the absence of acetic acid and EG, the same diffractogram as pure phase UiO-66 was produced, except with significant reduction in crystallinity which was evident from the XRD pattern. Thus, the proposed mechanism held true and was conclusive from the characterization results. Additional experiments were also performed to check for the reproducibility of these fibers; the SEM images and XRD patterns are included in Appendix A.3-1, A.3-2, and A.3-3. The results indicated high reproducibility of the sample in the presence of acetic acid. However, this assumption requires further analysis of the resultant UiO-66 particles through ${ }^{1} \mathrm{HNMR}$ and elemental analysis to determine the constitutive particles like the monocarboxylates 
that compensate for the defects within the framework. Additionally, the implementation of SAXS would be helpful in identifying the density of defects within the Ui0-66 sample.

\subsubsection{Conclusion}

As per the SEM and XRD results, it was evident that the presence of acetic acid in the solution was necessary to produce well-defined Ui0-66 crystals. The case was also true for the samples synthesized with 30 eq. EG as a co-solvent indicating that EG alone was insufficient to re-direct the growth of UiO-66 crystals from (111) plane to (200) plane. The effect of acetic acid as a modulator in the solution was profound as including the acetic acid in the reaction transformed the growth of Ui0-66 crystals from amorphous and ill-defined to highly crystalline UiO-66. Therefore, it can be concluded that a proper ratio between the modulator and co-solvent is necessary to yield crystalline materials. 


\section{CHAPTER FIVE}

\section{SUMMARY}

The aim of this project was achieved by investigating the effects of process and composition variables on the framework and structural properties of UiO-66. The first experiment was to minimize the energy requirement in the solvothermal synthesis process by conducting the study at room temperature. The results revealed that Ui0-66 was successfully formed at room temperature, however, the reaction kinetics were extremely slow with an approximate reaction time of 30 days. In addition, the crystallinity of the particles was subpar in comparison to the crystals synthesized at $120^{\circ} \mathrm{C}$. Thus, a reaction temperature of $120^{\circ} \mathrm{C}$ was used for the following syntheses.

The next experiment investigated the effect of stirring that had not been studied previously. It was found that the extent of stirring had a significant impact on the crystallinity of UiO-66. Stirring of the precursor solution helped in stabilizing the nuclei and appropriate aggregation of $\mathrm{Zr}$ and BDC complexes, thus, promoting formation of discrete crystals at $24 \mathrm{~h}$. However, extended stirring time, i.e., $>24 \mathrm{~h}$, damaged the crystallinity by breaking down the aggregates, resulting in small ill-defined particles with poor crystallinity.

The effect of reaction time was also studied by varying the reaction times from $12 \mathrm{~h}$ to $72 \mathrm{~h}$ with an extended stirring time of $48 \mathrm{~h}$. The prolonged stirring time ensured increased number of stabilized nuclei such that the particles were formed at shorter synthesis time of $12 \mathrm{~h}$. Thus, doubling the stirring time of the precursor solution reduced the crystallization

time to $24 \mathrm{~h}$. Additionally, a natural thermodynamically driven ADOR process was also observed for the first time in the case of MOFs with extended reaction time.

The next factor that was studied was the effect of $\mathrm{Zr}$ :BDC molar ratio by limiting the $\mathrm{Zr}$ metal and BDC individually in the precursor solution. By limiting the quantity on $\mathrm{Zr}$, an increase in the BDC concentration increased the particle size as fewer $\mathrm{Zr}$ atoms, being the core of the SBU naturally reduces their formation. The increased quantity of BDC produced particles of comparable crystallinity to the as-synthesized sample for $1: 1.5$ ration but reduced the crystallinity with $1: 2$ ratio. On the other hand, limiting the quantity of BDC reduced the 
linkages between the SBUs which in turn caused polydispersity in the. Moreover, reduction in BDC caused more defects in the crystals leading to increased particle size.

Another important factor that was studied was the effect of reaction temperature on the morphology of UiO-66 crystals. A synthesis temperature of $100^{\circ} \mathrm{C}$ at $48 \mathrm{~h}$ reaction time proved to be insufficient in producing discrete crystals. This was because at low synthesis temperature, the reactants had insufficient energy to cross the activation barrier leading to small ill-defined morphologies. On increasing the temperature to $120^{\circ} \mathrm{C}$ at $24 \mathrm{~h}$ (assynthesized), particles became more discrete with octahedral shaped crystals due to the sufficient kinetic energy of the reactant molecules. With higher reaction temperature, i.e., $140^{\circ} \mathrm{C}$ at $16 \mathrm{~h}$, particles were formed at shorter reaction time as molecules had the highest kinetic energy at this temperature. However, the crystallinity of this sample was lower than the as-synthesized sample as higher reaction temperature produced defects in the crystal structure due to the increased lability of Zr-O bond.

With the modulator being the strongest structure directing factor in the synthesis process, the concentration of modulator was varied from $0.0103 \mathrm{~mol}$ to $0.12 \mathrm{~mol}$. Increasing the acetic acid concentration from $0.0103 \mathrm{~mol}$ to $0.03 \mathrm{~mol}$ increased the particle size as well as the crystallinity of the UiO-66 sample but also introduced surface defects. This was because the increased quantity of acetic acid in the precursor solution produced SBUs with more of the deprotonated acetic acid. As a result, crystals were produced with higher defects, thus, increasing the surface area. However, further increase in acetic acid content damaged the crystallinity and morphology of the particles due to higher concentration of missing linker defects.

The last part of the study involved the addition of co-solvents, i.e., EG, DEG and TEG, to the reactant mixture in an attempt to modify the morphology of UiO-66 crystals. Introduction of 30 eq. EG successfully changed the morphology of UiO-66 crystals from traditional octahedral crystals to needle shaped fibers. This was because of the formation of in-situ ligand BHET that was able to induce directional growth of UiO-66 crystals from (111) orientation to (200) orientation. Moreover, this reorientation was more evident when the EG quantity was gradually increased from 5 eq. to 50 eq. With the introduction of DEG and 
TEG, the increased $\mathrm{pH}$ of the solution caused heightened deprotonation of acetic acid, similar to the effect of increased modulator concentration, except this effect was much stronger. The deprotonated acetic acid bound to the $\mathrm{Zr}$ acid moieties to produce large octahedral crystals with an increased particle size from $\sim 0.2-0.1 \mu \mathrm{m}$. As the difference in $\mathrm{p} K_{\mathrm{a}}$ values of DEG and TEG were negligible, the difference in the crystallinity was explained by their significant difference in viscosities. DEG being lower in viscosity allowed the interaction of its constituent solute particles, while TEG being higher in viscosity acted as a hindrance in the formation of the $\mathrm{Zr}$-acetate complexes despite the increased deprotonated acetate molecules. Furthermore, on increasing the DEG quantity from 10 eq. - 30 eq., the particles became monodisperse. This was because lower quantity of DEG was insufficient in inducing heightened deprotonation of acetic acid. Contrastingly, changing the concentration of TEG from 5 eq. - 30 eq., the samples remained polydisperse throughout the various concentrations. This was attributed to its high viscosity that made the formation of $\mathrm{Zr}$ acetate and BDC molecules a challenging process. 


\section{CHAPTER SIX \\ RECOMMENDATIONS}

The literature survey and all the above performed experiments proved that each reaction factor affects the framework of the MOF differently. Altering both compositional and process variables caused a difference in both the structure and morphology of UiO-66 crystals. After gaining an in-depth understanding of each of these variables, and among the various reported studies, it was safely concluded that each MOF underwent distinct structural change under diverse conditions. Moreover, following a synthesis recipe, the variables can be altered to select an optimal reaction condition to produce large UiO-66 crystals. Among the compositional factors, judicious choices of solvents and modulators were observed to have the most significant effect in terms of the manipulating the structure of the MOF. The examples suggested that solvent indirectly affects the infrastructure of MOFs by regulating the rate of nucleation. This process aided in self-assembly process of coordination bonding between metal and ligands to produce diverse morphologies of UiO-66. However, the results can be optimized by introducing several more concentrations of both co-solvent and modulator to determine the exact concentration required to build MOFs to its full potential. For the first time, the effect of stirring time on the morphology of UiO-66 was confirmed through a systematic alteration of stirring time. As the strength of stirring in these experiments were kept constant, the study also opened up the possibility of the effect of stirring speed as they have a significant impact on these frameworks [139]. that Moreover, the new morphologies obtained using EG as a co-solvent should further be assessed using ${ }^{1} \mathrm{HNMR}$ and elemental analysis to figure out the constitutive particles like the monocarboxylates that compensate for the defects within the framework. Additionally, the implementation of SAXS would be helpful in identifying the density of defects within the UiO66 sample as XRD readily indicates this through forbidden reflections at lower angle between $4^{\circ}-7^{\circ}$ of the $2 \theta$ diffraction angle [140], [141]. In addition, the introduction of modulators and co-solvents certainly affects the $\mathrm{pH}$ of the solution, which is another characteristic factor that needs to be addressed in the formation of MOFs. The change in $\mathrm{pH}$ of the solution affects the dimensionality of the product [142], rate of crystallization, ligand transformation, degree of deprotonation of organic ligands, and metal ligand coordination 
that directly control MOFs' structure [104], [143]. Depending on the acidity/basicity of the reaction medium, it regulates the deprotonation of organic ligand in aqueous solutions [104]. Thus, studying the effect of $\mathrm{pH}$ can also help us understand the medium required for the growth of these crystals.

In addition, the occurrence of recrystallization-dissolution process was observed for the first time in UiO-66 with variation in stirring time, as well as the reaction time. The results suggested that there may be multiple maximum points in terms of the crystallinity of the samples. It was also difficult to pin-point the governing mechanisms taking place in the formulation of the UiO-66 crystals. Thus, it is recommended that the crystallinity of the sample be evaluated at longer and shorter reaction and stirring times, possibly with in-situ XRD technique and other sophisticated techniques such as DLS (dynamic light scattering) to detect the start of nucleation and growth process by detecting the size of the associates.

The application of an appropriate temperature depends upon the requirement of the desired characteristics of the MOF as it influences the dimensionality, solubility, and coordination modes of metal centers and ligands. The stoichiometric ratio of the reactants also displays a similar existence of optimal condition that renders it economical to usually have a 1:1 metal:linker ratio.

Hence, the tailorability of MOFs is beyond fine-tuning procedure and is more complex than one may perceive. Once the process and composition variables are combined, it becomes a challenge to pinpoint the underlying mechanics within the synthesis process. Therefore, the missing information makes the reproducibility variation of crystal morphology and structure, in terms of synthesis batch and personnel a challenging task which requires further study. Nevertheless, the results from this project provide a useful insight on designing MOFs by altering the available variables via solvothermal synthesis process which makes its fabrication a little less unpredictable. The comprehensive study of each one of these factors in the solvothermal synthesis of UiO-66 can help determine the ideal conditions to design frameworks through systematic tuning of the available process and compositional variables for this synthesis process to design such frameworks. If better surface areas are produced, the material can be utilized for gas separation and other diverse applications. 


\section{APPENDICES}

APPENDIX A: Effect of reaction time at $140^{\circ} \mathrm{C}$ with 24 hours of stirring via Scheme 1

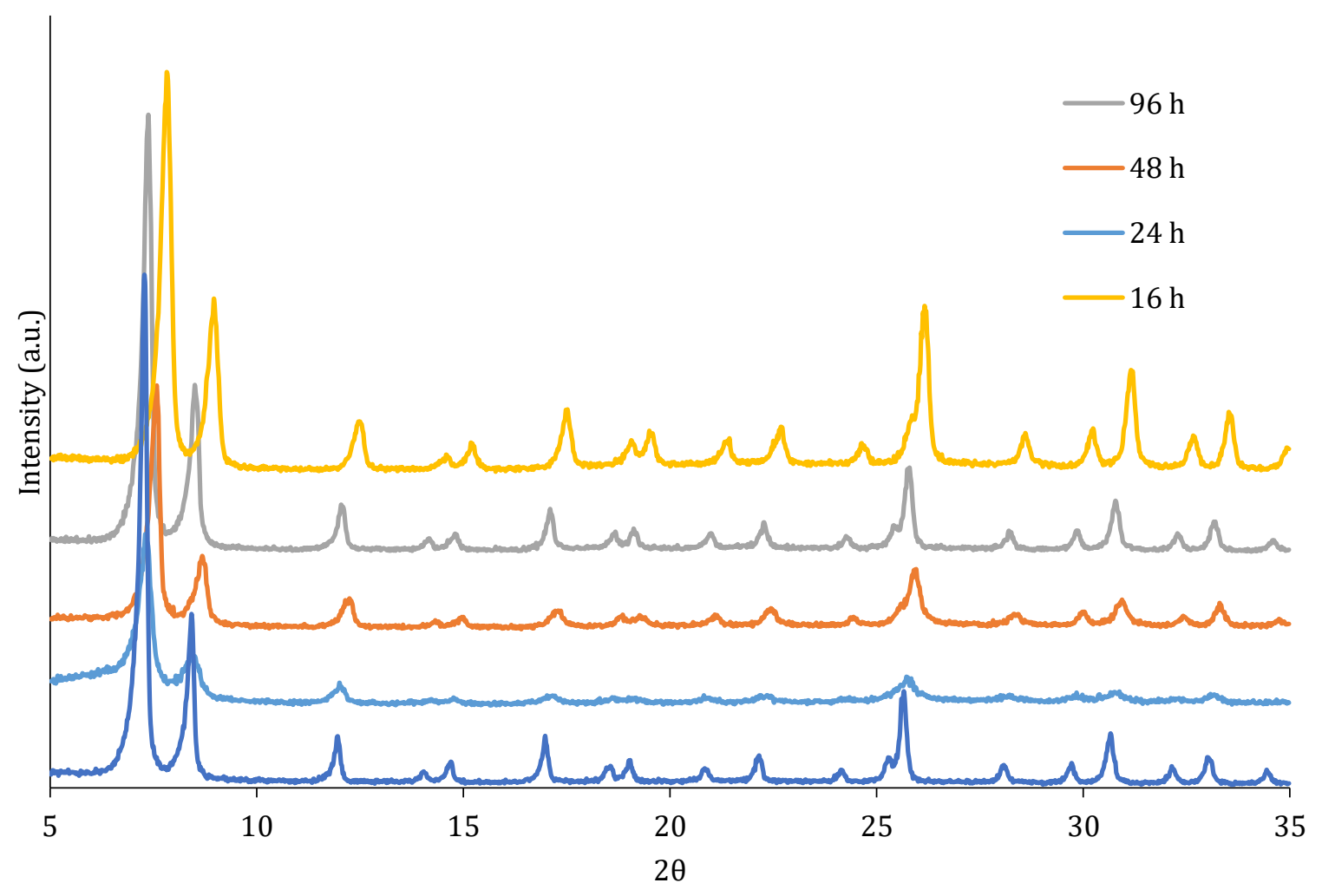

Figure A-1: XRD patterns on varying reaction times at $140^{\circ} \mathrm{C}$. 


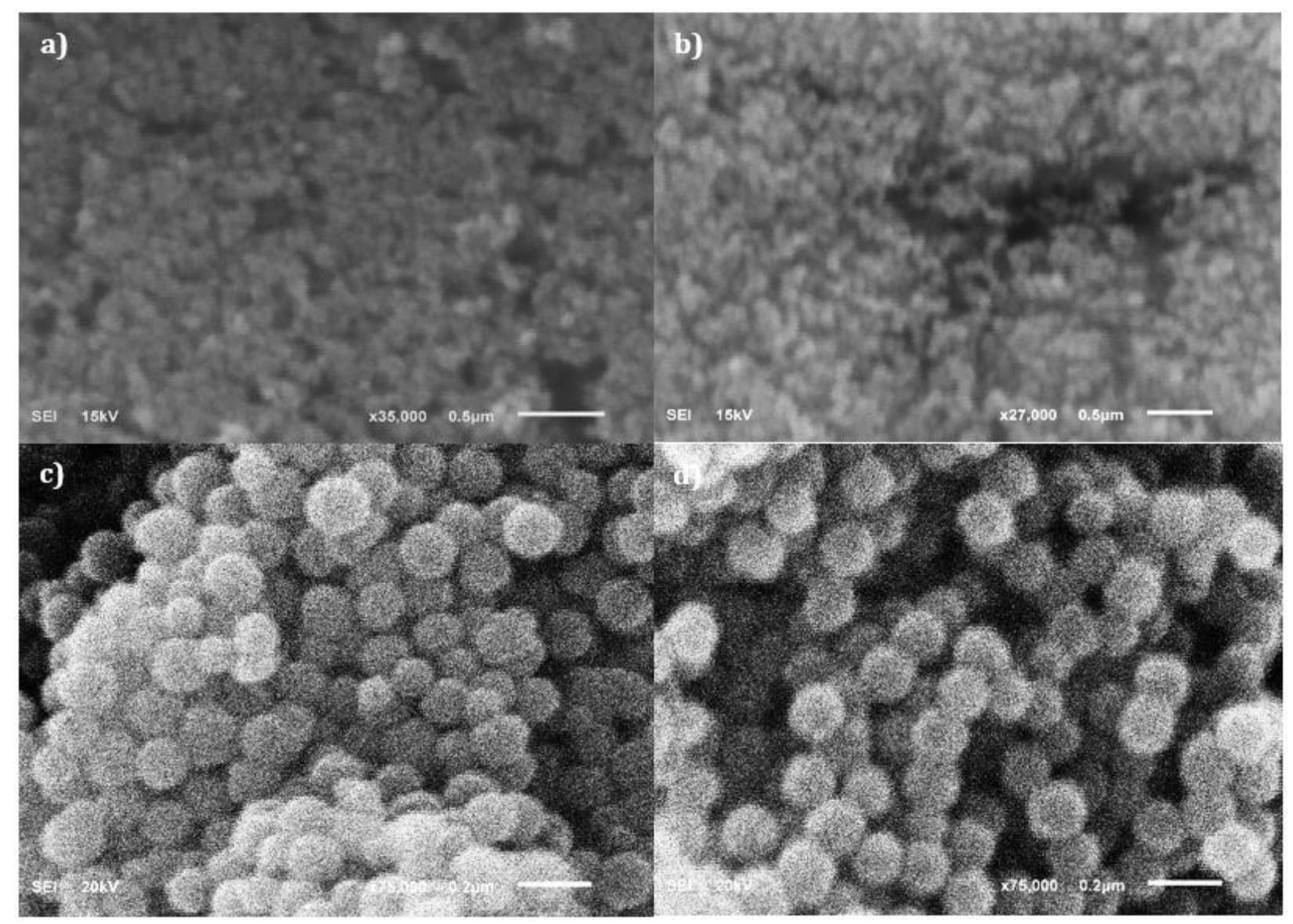

Figure A-2: SEM images of UiO- 66 at $140^{\circ} \mathrm{C}$ with reaction times of a) $12 \mathrm{~h}$, b) $24 \mathrm{~h}$ c) $16 \mathrm{~h}$, and d) as-synthesized. 
APPENDIX B: Effect of 30 eq. co-solvents with $24 \mathrm{~h}$ stirring at $120^{\circ} \mathrm{C}$ for $96 \mathrm{~h}$ via Scheme 2

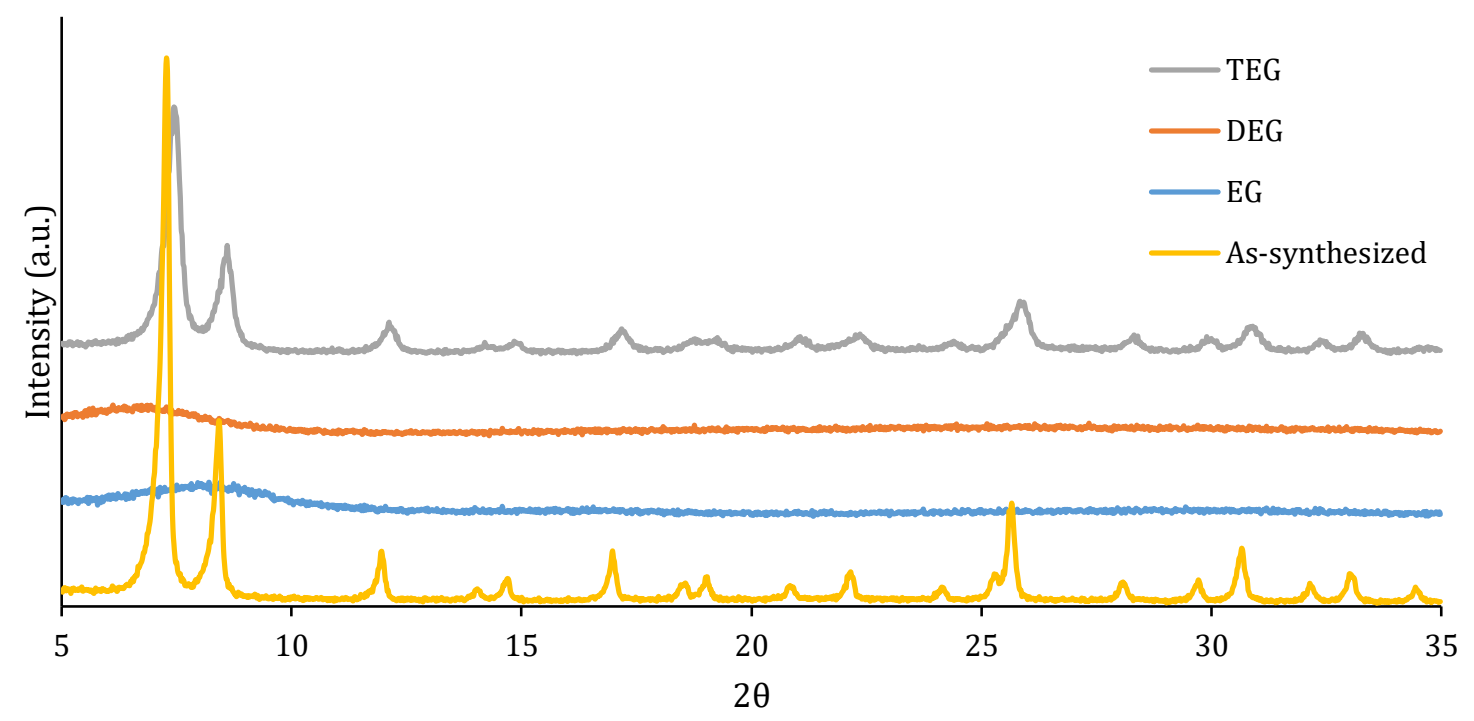

Figure B-1: XRD patterns of UiO- 66 at $120^{\circ} \mathrm{C}$ for $96 \mathrm{~h}$ with 30 eq. of various glycols. 


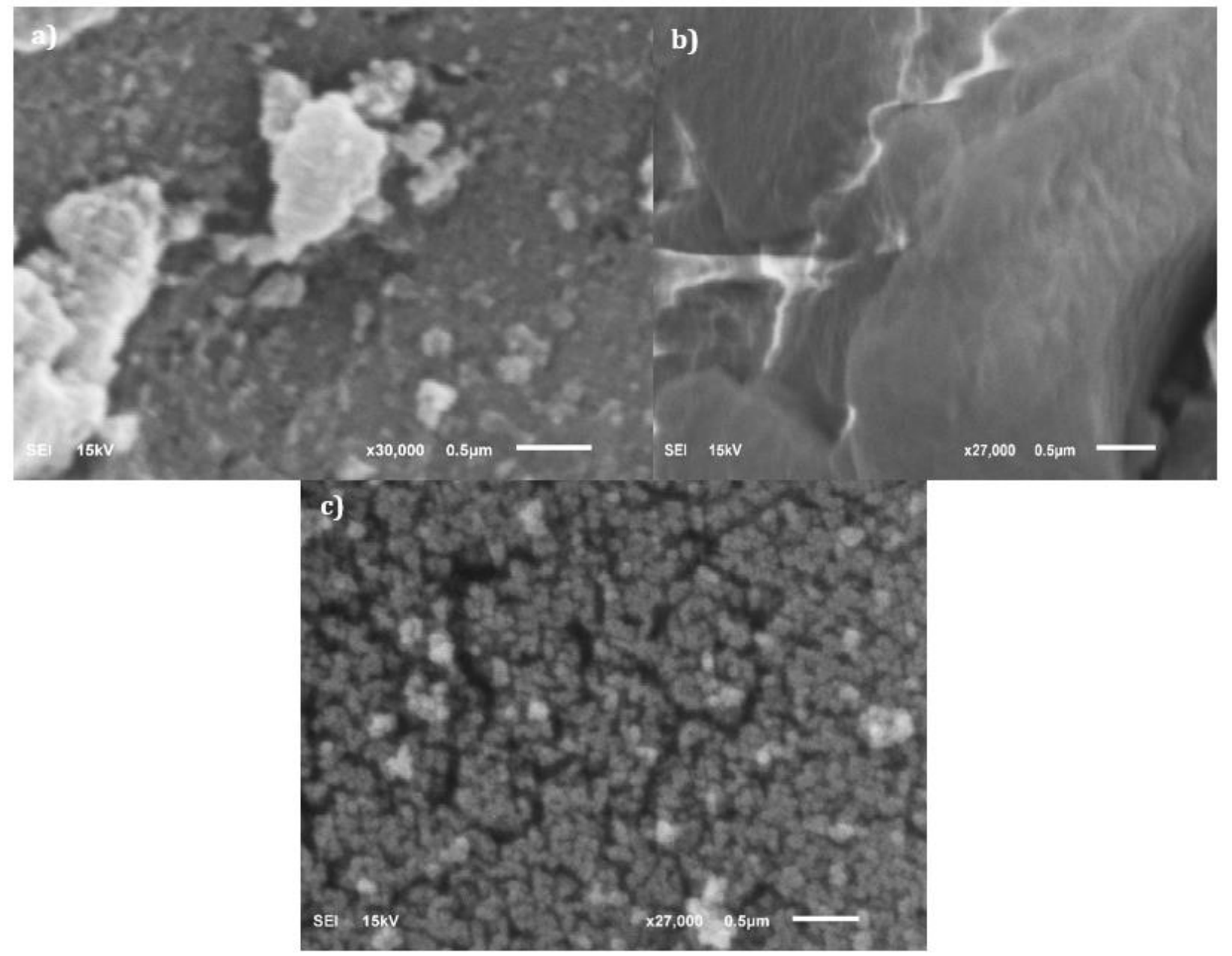

Figure B-2: SEM images of UiO- 66 at $120^{\circ} \mathrm{C}$ for $96 \mathrm{~h}$ with 30 eq. of a) EG, b) DEG, and c) TEG. 
APPENDIX C: Reproducibility of needle-shaped fibers using EG as a co-solvent with 24h stirring at $120^{\circ} \mathrm{C}$ for $96 \mathrm{~h}$ via Scheme 3

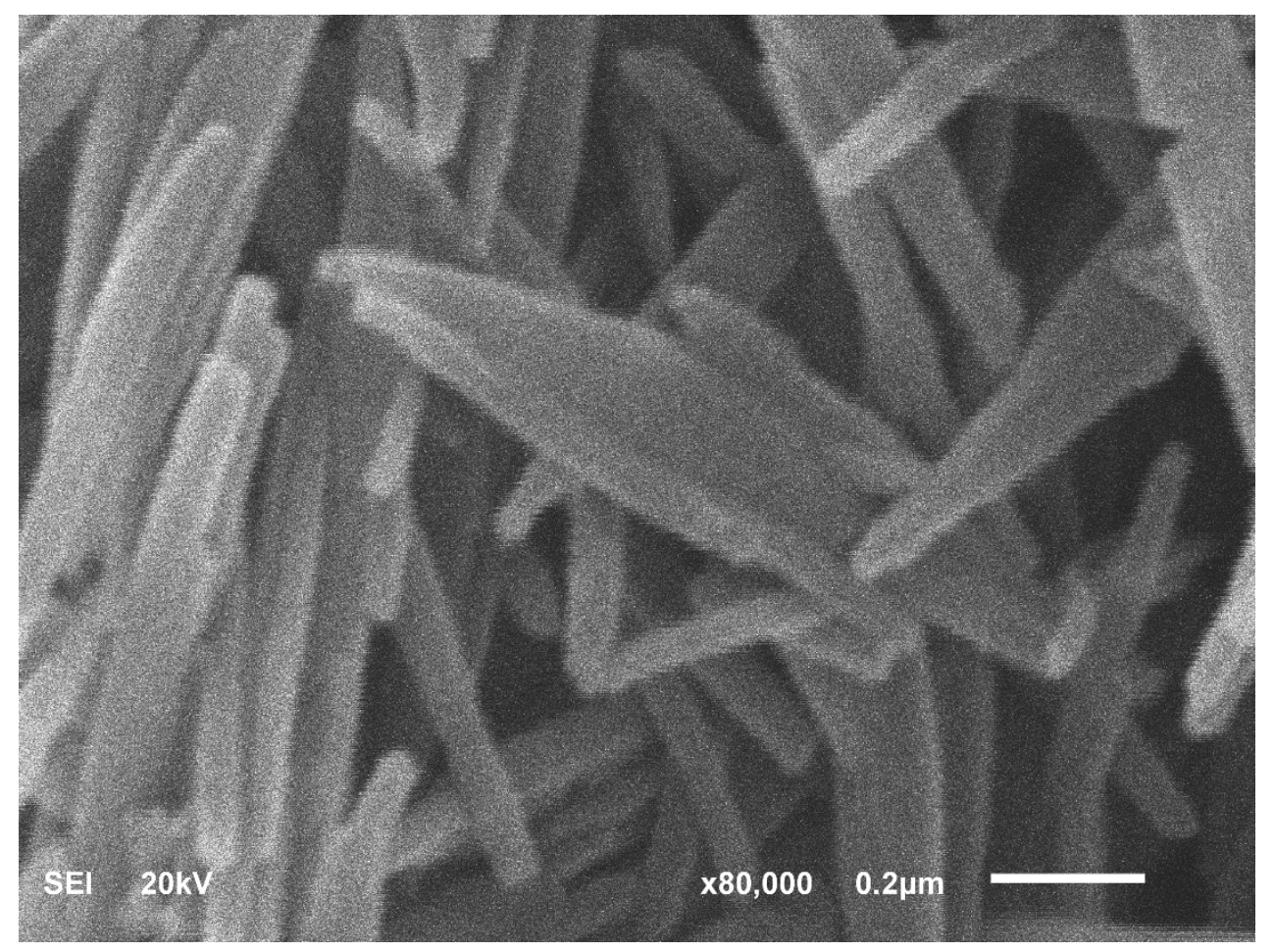

Figure C-1: SEM image of UiO- 66 at $120^{\circ} \mathrm{C}$ for $96 \mathrm{~h}$ using 30 eq. EG co-solvent.

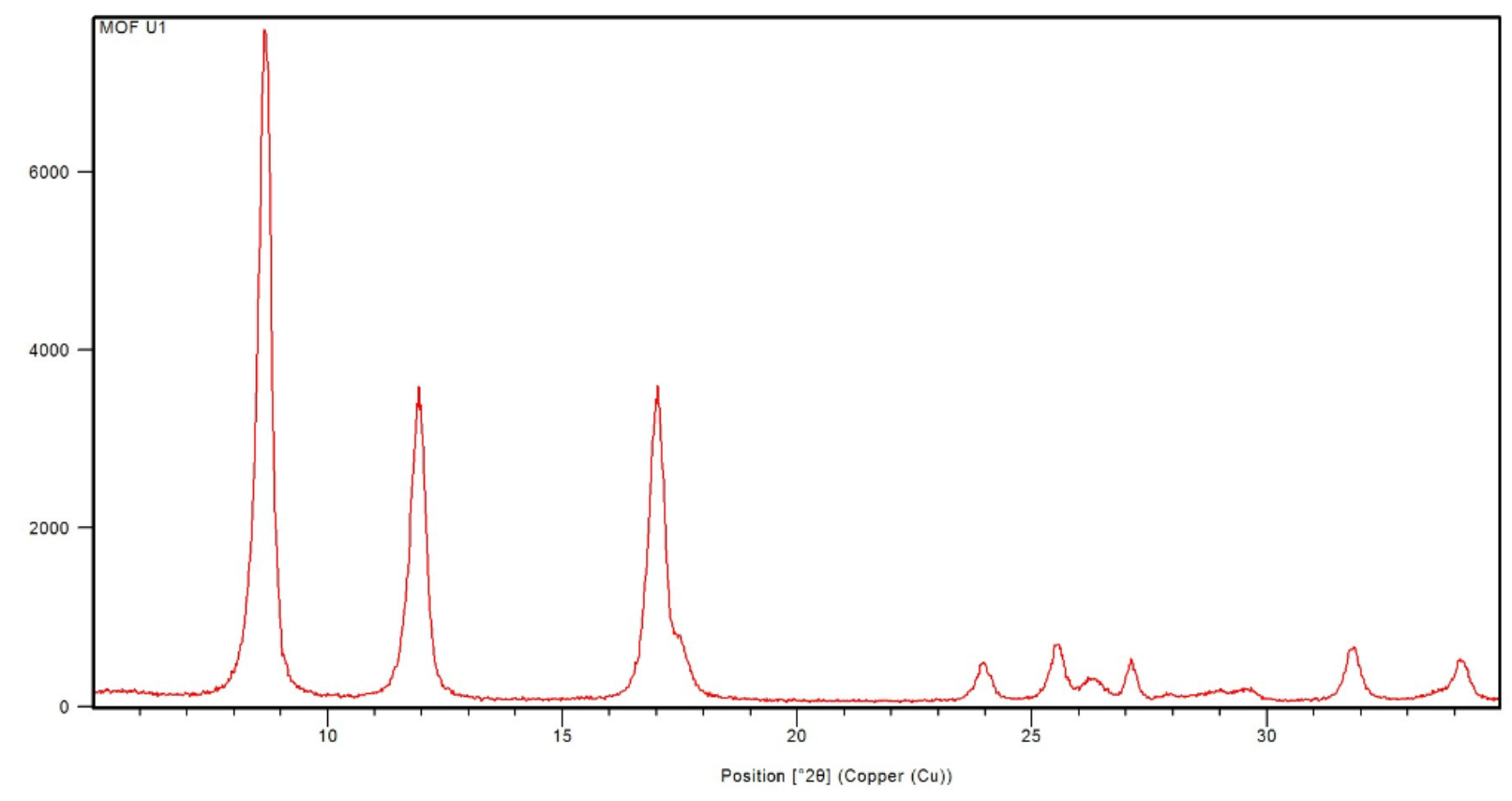

Figure C-2: XRD image of UiO-66 at $120^{\circ} \mathrm{C}$ for $96 \mathrm{~h}$ using 30 eq. EG co-solvent. 


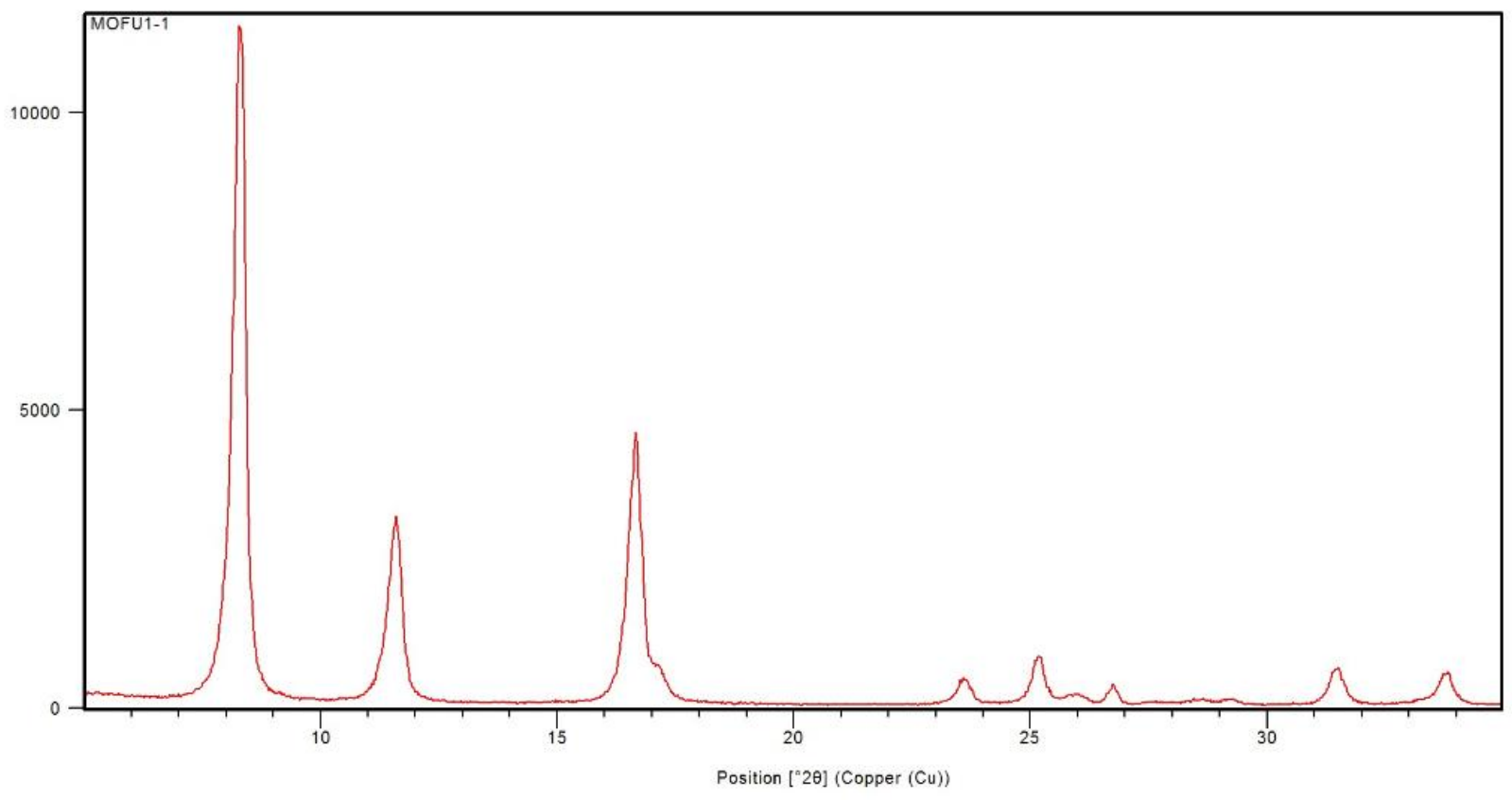

Figure C-3: XRD image of UiO-66 at $120^{\circ} \mathrm{C}$ for $96 \mathrm{~h}$ using 30 eq. EG co-solvent. 


\section{REFERENCES}

[1] Z. Hu and D. Zhao, "De facto methodologies toward the synthesis and scale-up production of UiO-66-type metal-organic frameworks and membrane materials," Dalt. Trans., vol. 44, no. 44, pp. 19018-19040, 2015.

[2] H. Li, M. Eddaoudi, M. O'Keeffe, and O. M. Yaghi, "Design and synthesis of an exceptionally stable and highly porous metal-organic framework," Nature, vol. 402, no. November, pp. 276-279, 1999.

[3] Z. Hu, M. Khurana, Y. H. Seah, M. Zhang, Z. Guo, and D. Zhao, "Ionized Zr-MOFs for highly efficient post-combustion $\mathrm{CO}_{2}$ capture," Chem. Eng. Sci., vol. 124, pp. 61-69, 2015.

[4] Y. Bai, Y. Dou, L.-H. Xie, W. Rutledge, J.-R. Li, and H.-C. Zhou, "Zr-based metal-organic frameworks: design, synthesis, structure, and applications," Chem. Soc. Rev., vol. 45, no. 8, pp. 2327-2367, 2016.

[5] H. R. Abid, H. M. Ang, and S. Wang, "Effects of ammonium hydroxide on the structure and gas adsorption of nanosized Zr-MOFs (UiO-66)," Nanoscale, vol. 4, no. 10, p. 3089, 2012.

[6] H. Furukawa, K. E. Cordova, M. O'Keeffe, and O. M. Yaghi, "The chemistry and applications of metal-organic frameworks," Science, vol. 341, no. 6149, pp. 12304441-1230444-12, 2013.

[7] D. Wu, G. Maurin, Q. Yang, C. Serre, H. Jobic, and C. Zhong, "Computational exploration of a Zr-carboxylate based metal-organic framework as a membrane material for $\mathrm{CO}_{2}$ capture," J. Mater. Chem. A, vol. no.2, no. 2, pp. 1657-1661, 2014.

[8] B. F. Hoskins and R. Robson, "Infinite polymeric frameworks consisting of three dimensionally linked rod-like segments," J. Am. Chem. Soc., vol. 111, no. 15, pp. 59625964, 1989.

[9] M. Alhamami, H. Doan, and C. H. Cheng, "A review on breathing behaviors of metalorganic-frameworks (MOFs) for gas adsorption," Materials, vol. 7, no. 4, pp. 31983250, 2014.

[10] P. Deria, Y. G. Chung, R. Q. Snurr, J. T. Hupp, and O. K. Farha, "Water stabilization of Zr6 -based metal-organic frameworks via solvent-assisted ligand incorporation," Chem. Sci., vol. 6, no. 9, pp. 5172-5176, 2015.

[11] P. Deria, J. E. Mondloch, O. Karagiaridi, W. Bury, J. T. Hupp, and O. K. Farha, "Beyond post-synthesis modification: evolution of metal-organic frameworks via building block replacement," Chem. Soc. Rev., vol. 43, no. 16, pp. 5896-5912, 2014.

[12] G. Férey, C. Mellot-Draznieks, C. Serre, F. Millange, J. Dutour, S. Surblé, and I. Margiolaki, "A chromium terephthalate-based solid with unusually large pore volumes and surface area," Am. Assoc. Adv. Sci., vol. 326, no. 5952, pp. 535-538, 2018. 
[13] Y. R. Lee, J. Kim, and W. S. Ahn, "Synthesis of metal-organic frameworks: A mini review," Korean J. Chem. Eng., vol. 30, no. 9. pp. 1667-1680, 2013.

[14] A. Torkkeli, "Droplet microfluidics on a planar surface," VTT Publ., vol. 59, no. 504, pp. 3-194, 2003.

[15] Y. R. Lee, M. S. Jang, H. Y. Cho, H. J. Kwon, S. Kim, and W. S. Ahn, "ZIF-8: A comparison of synthesis methods," Chem. Eng. J., vol. 271, pp. 276-280, 2015.

[16] M. Almáši, V. Zeleňák, P. Palotai, E. Beňová, and A. Zeleňáková, "Metal-organic framework MIL-101(Fe)- $\mathrm{NH}_{2}$ functionalized with different long-chain polyamines as drug delivery system," Inorg. Chem. Commun., vol. 93, no. 2017, pp. 115-120, 2018.

[17] T. C. Wang, N. A Vermeulen, I. S. Kim, A.B. F. Martinson, J F. Stoddart, J.T. Hupp, and O. K. Farha, "Scalable synthesis and post-modification of a mesoporous metal-organic framework called NU-1000," Nat. Protoc., vol. 11, no. 1, pp. 149-162, 2016.

[18] P. Deria, W. Bury, J. T. Hupp, and O. K. Farha, "Versatile functionalization of the NU1000 platform by solvent-assisted ligand incorporation," Chem. Commun., vol. 50, no. 16, pp. 1965-1968, 2014.

[19] J. Goldsmith, A. G. Wong-Foy, M. J. Cafarella, and D. J. Siegel, "Theoretical limits of hydrogen storage in metal-organic frameworks: Opportunities and trade-offs," Chem. Mater., vol. 25, no. 16, pp. 3373-3382, 2013.

[20] M. A. K. Khalil and R. A. Rasmussen, "Global increase of atmospheric molecular hydrogen," Nature, vol. 347, no. 2, pp. 743-745, 1990.

[21] U. Schmidt, "Molecular hydrogen in the atmosphere," Tellus, vol. 26, no. 1-2, pp. 7890, 2012.

[22] J. D. Shakun P. U. Clark, F. He, S. A. Marcott, A.C. Mix, Z. Liu, B. Otto-Bliesner, A. Schmittner, and E. Bard, "Global warming preceded by increasing carbon dioxide concentrations during the last deglaciation," Nature, vol. 484, no. 7392, pp. 49-54, 2012.

[23] H. R. Abid, G. H. Pham, H. M. Ang, M. O. Tade, and S. Wang, "Adsorption of $\mathrm{CH}_{4}$ and $\mathrm{CO}_{2}$ on Zr-metal organic frameworks," J. Colloid Interf. Sci., vol. 366, no. 1, pp. 120-124, 2012.

[24] Z. Hu, S. Faucher, Y. Zhuo, Y. Sun, S. Wang, and D. Zhao, "Combination of optimization and metalated-ligand exchange: An effective approach to functionalize UiO-66(Zr) MOFs for $\mathrm{CO}_{2}$ separation," Chem. - A Eur. J., vol. 21, no. 48, pp. 17246-17255, 2015.

[25] P. Horcajada T. Chalati, C. Serre, B. Gillet, C. Sebrie, T. Baati, J. F. Eubank, D. Heurtaux, P. Clayette, C. Kreuz, J. Chang, Y. K. Hwang, V. Marsaud, P. Bories, L. Cynober, S. Gil, G. Férey, P. Couvreur, and R. Gref, "Porous metal-organic-framework nanoscale carriers as a potential platform for drug delivery and imaging," Nat. Mater., vol. 9, no. 2, pp. 172-178, 2010. 
[26] J. Zhang and K. Cai, "Integration of polymers in the pore space of mesoporous nanocarriers for drug delivery," J. Mater. Chem. B, vol. 5, no. 45, pp. 8891-8903, 2017.

[27] M. Filippousi, S. Turner, K. Leus, P. I. Siafaka, E. D. Tseligka, M. Vandichel, S. G. Nanaki, I. S. Vizirianakis, D. N. Bikiaris, P. V. D. Voort, and G. V. Tendeloo, "Biocompatible Zrbased nanoscale MOFs coated with modified poly( $\varepsilon$-caprolactone) as anticancer drug carriers," Int. J. Pharm., vol. 509, no. 1-2, pp. 208-218, 2016.

[28] D. Cunha M. B. Yahia, S. Hall, S. R. Miller, H. Chevreau, E. Elkaïm, G. Maurin, P. Horcajada, and C. Serre, "Rationale of drug encapsulation and release from biocompatible porous metal-organic frameworks," Chem. Mater., vol. 25, no. 14, pp. 2767-2776, 2013.

[29] S. Rojas I. Colinet, D. Cunha, T. Hidalgo, F. Salles, C. Serre, N. Guillou, and P. Horcajada, "Toward Understanding Drug Incorporation and Delivery from Biocompatible MetalOrganic Frameworks in View of Cutaneous Administration," ACS Omega, vol. 3, no. 3, pp. 2994-3003, 2018.

[30] X. Zhu, J. Gu, Y. Wang, B. Li, Y. Li, W. Zhao, and J. Shi, "Inherent anchorages in UiO-66 nanoparticles for efficient capture of alendronate and its mediated release," Chem. Commun., vol. 50, no. 63, pp. 8779-8782, 2014.

[31] U. S. F. Arrozi, H. W. Wijaya, A. Patah, and Y. Permana, "Efficient acetalization of benzaldehydes using UiO-66 and UiO-67: Substrates accessibility or Lewis acidity of zirconium," Appl. Catal. A-Gen., vol. 506, pp. 77-84, 2015.

[32] J. Long S. Wang, Z. Ding, S. Wang, Y. Zhou, L. Huang, and X. Wang, "Aminefunctionalized zirconium metal-organic framework as efficient visible-light photocatalyst for aerobic organic transformations," Chem. Commun., vol. 48, no. 95, p. 11656-11658, 2012.

[33] F. Zhou, N. Lu, B. Fan, H. Wang, and R. Li, "Zirconium-containing UiO-66 as an efficient and reusable catalyst for transesterification of triglyceride with methanol," J. Energy Chem., vol. 25, no. 5, pp. 874-879, 2016.

[34] J. F. Blandez A. Santiago-Portillo, S. Navalón, M. Giménez-Marqués, M. Álvaro, P. Horcajada, and H. García, "Influence of functionalization of terephthalate linker on the catalytic activity of UiO-66 for epoxide ring opening," J. Mol. Catal. A-Chem., vol. 425, pp. 332-339, 2016.

[35] F. Vermoortele B. Bueken, G. L. Bars, B. V. D. Voorde, M. Vandichel, K. Houthoofd, A. Vimont, M. Daturi, M. Waroquier, V. V. Speybroeck, C. Kirschhock, and D.E. De Vos, "Synthesis modulation as a tool to increase the catalytic activity of metal-organic frameworks: The unique case of Ui0-66(Zr)," J. Am. Chem. Soc., vol. 135, no. 31, pp. 11465-11468, 2013.

[36] M. Bosch, S. Yuan, and H. Zhou, "Group 4 metals as Secondary Building Units: Ti, Zr, and Hf-based MOFs," in The Chemistry of Metal-Organic Frameworks: Synthesis, Characterization, and Applications, 1st ed., S. Kaskel, Ed. 2016, pp. 137-170. 
[37] J. H. Cavka, S. Jakobsen, U. Olsbye, N. Guillou, S. Bordiga, and K. P. Lillerud, "A New Zirconium Inorganic Building Brick Forming Metal Organic Frameworks with Exceptional Stability - Journal of the American Chemical Society (ACS Publications)," vol. no. 130, no. 42, pp. 13850-13851, 2008.

[38] A. Schaate P. Roy, A. Godt, J. Lippke, F. Waltz, M. Wiebcke, and P. Behrens, "Modulated synthesis of Zr-based metal-organic frameworks: From nano to single crystals," Chem. - A Eur. J., vol. 17, no. 24, pp. 6643-6651, 2011.

[39] S. Yuan, J. S. Qin, C. T. Lollar, and H. C. Zhou, "Stable Metal-Organic Frameworks with Group 4 Metals: Current Status and Trends," ACS Cent. Sci., Vol. no. 4, no. 4, pp. 440450, 2018.

[40] H. Wu, T. Yildirim, and W. Zhou, "Exceptional mechanical stability of highly porous zirconium metal-organic framework UiO-66 and its important implications," J. Phys. Chem. Lett., vol. 4, no. 6, pp. 925-930, 2013.

[41] L. Valenzano, B. Civalleri, S. Chavan, S. Bordiga, M. H. Nilsen, S. Jakobsen, K. P. Lillerud and C. Lamberti, "Disclosing the complex structure of UiO-66 metal organic framework: A synergic combination of experiment and theory," Chem. Mater., vol. 23, no. 7, pp. 1700-1718, 2011.

[42] L. Xia and F. Wang, "Prediction of hydrogen storage properties of Zr-based MOFs," Inorganica Chim. Acta, vol. 444, pp. 186-192, 2016.

[43] N. Stock and S. Biswas, "Synthesis of metal-organic frameworks (MOFs): Routes to various MOF topologies, morphologies, and composites," Chem. Rev., vol. 112, no. 2, pp. 933-969, 2012.

[44] J. Klinowski, F. A. Almeida Paz, P. Silva, and J. Rocha, "Microwave-Assisted Synthesis of Metal-Organic Frameworks," Dalt. Trans., vol. 40, no. 22, pp. 321-330, 2011.

[45] A. J. Howarth, A. W. Peters, N. A. Vermeulen, T. C. Wang, J. T. Hupp, and O. K. Farha, "Best Practices for the Synthesis, Activation, and Characterization of Metal-Organic Frameworks," Chem. Mater., vol. 29, no. 1, pp. 26-39, 2017.

[46] C. Vaitsis, G. Sourkouni, and C. Argirusis, "Metal organic frameworks (MOFs) and ultrasound: A review," Ultrason. Sonochem., vol. 52, pp. 106-119, 2018.

[47] S. Asgharzadehahmadi, A. A. Abdul Raman, R. Parthasarathy, and B. Sajjadi, "Sonochemical reactors: Review on features, advantages and limitations," Renew. Sustain. Energy Rev., vol. 63, no. 1-603, pp. 302-314, 2016.

[48] S. Sachdeva, A. Pustovarenko, E. J. R. Sudhölter, F. Kapteijn, L. C. P. M. De Smet, and J. Gascon, "Control of interpenetration of copper-based MOFs on supported surfaces by electrochemical synthesis," Cryst. Eng. Comm., vol. 18, no. 22, pp. 4018-4022, 2016.

[49] A. Martinez Joaristi, J. Juan-Alcañiz, P. Serra-Crespo, F. Kapteijn, and J. Gascon, "Electrochemical synthesis of some archetypical $\mathrm{Zn}^{2+}, \mathrm{Cu}^{2+}$, and $\mathrm{Al}^{3+}$ metal organic frameworks," Cryst. Growth Des., vol. 12, no. 7, pp. 3489-3498, 2012. 
[50] I. Stassen M. Styles, T. V. Assche, N. Campagnol, J. Fransaer, J. Denayer, J. Tan, P. Falcaro, D. D. Vos, and R. Ameloot, "Electrochemical film deposition of the zirconium metal-organic framework UiO-66 and application in a miniaturized sorbent trap," Chem. Mater., vol. 27, no. 5, pp. 1801-1807, 2015.

[51] K. Pirzadeh, A. A. Ghoreyshi, M. Rahimnejad, and M. Mohammadi, "Electrochemical synthesis, characterization and application of a microstructure $\mathrm{Cu}_{3}(\mathrm{BTC})_{2}$ metal organic framework for $\mathrm{CO}_{2}$ and $\mathrm{CH}_{4}$ separation," Korean J. Chem. Eng., vol. 35, no. 4, pp. 974-983, 2018.

[52] M. Klimakow, P. Klobes, A. F. Thünemann, K. Rademann, and F. Emmerling, "Mechanochemical synthesis of metal-organic frameworks: A fast and facile approach toward quantitative yields and high specific surface areas," Chem. Mater., vol. 22, no. 18, pp. 5216-5221, 2010.

[53] A. L. Garay, A. Pichon, and S. L. James, "Solvent-free synthesis of metal complexes," Chem. Soc. Rev., vol. 36, no. 6, pp. 846-855, 2007.

[54] C. Mottillo and T. Friščić, "Advances in solid-state transformations of coordination bonds: From the ball mill to the aging chamber," Molecules, vol. 22, no. 1, pp. 1-38, 2017.

[55] A. M. Z. Slawin D.W. Aldous, R_ J. Goff, A. M. Z. Slawin, J. P. Attfield, R. E. Morris, and Pa Lightfoot, "An ionothermally prepared $S=1 / 2$ vanadium oxyfluoride kagome lattice," Nat. Chem., vol. 3, pp. 801-806, 2011.

[56] R. E. Morris, "Ionothermal synthesis - Ionic liquids as functional solvents in the preparation of crystalline materials," Chem. Commun., pp. 2990-2998, 2009.

[57] E. Ahmed and M. Ruck, "Ionothermal synthesis of polyoxometalates," Angew. Chem. Int. Ed., vol. 51, no. 2, pp. 308-309, 2012.

[58] Z. F. Wu, M. L. Feng, B. Hu, B. Tan, and X. Y. Huang, "Ionothermal synthesis of a metalorganic framework constructed by magnesium(II) and 4,4'-oxybis(benzoic acid) ligand," Inorg. Chem. Commun., vol. 24, pp. 166-169, 2012.

[59] S. Lin, W. Liu, Y. Li, Q. Wu, E. Wang, and Z. Zhang, "Preparation of polyoxometalates in ionic liquids by ionothermal synthesis," Dalt. Trans., vol. 39, no. 7, pp. 1740-1744, 2010.

[60] E. R. Cooper, C. D. Andrews, P. S. Wheatley, P. B. Webb, P. Wormald, and R. E. Morris, "Ionic liquids and eutectic mixtures as solvent and template in synthesis of zeolite analogues," Nature, vol. 430, pp. 1012-1016, 2004.

[61] X. Zhou, X. Lu, Z. Li, M. Zhu, and Q. Wang, "Effective catalysis of poly(ethylene terephthalate) (PET) degradation by metallic acetate ionic liquids," Pure Appl. Chem., vol. 84, no. 3, pp. 789-801, 2012.

[62] X. Sang, J. Zhang, J. Cui, L. Zheng, Z. Wu, Z. Li, G. Mo, Y. Xu, C. Liu, X. Tan, T. Luo, B. Zhang, and B. Han, "Ionic liquid accelerates the crystallization of Zr-based metal-organic frameworks," Nat. Commun., vol. 8, no. 1, pp. 1-7, 2017. 
[63] E. R. Parnham and R. E. Morris, "Ionothermal Synthesis of Zeolites, Metal - Organic Frameworks , and Inorganic - Organic Hybrids," Acc. Chem. Res., vol. 40, no. 10, pp. 1005-1013, 2007.

[64] D. J. Tranchemontagne, J. R. Hunt, and O. M. Yaghi, "Room temperature synthesis of metal-organic frameworks: MOF-5, MOF-74, MOF-177, MOF-199, and IRMOF-0," Tetrahedron, vol. 64, no. 36, pp. 8553-8557, 2008.

[65] Q. Liu, J. M. Yang, L. N. Jin, and W. Y. Sun, "Controlled synthesis of porous coordinationpolymer microcrystals with definite morphologies and sizes under mild conditions," Chem. - A Eur. J., vol. 20, no. 45, pp. 14783-14789, 2014.

[66] D. J. Tranchemontagne, J. R. Hunt, and O. M. Yaghi, "Room temperature synthesis of metal-organic frameworks: MOF-5, MOF-74, MOF-177, MOF-199, and IRMOF-0," Tetrahedron, vol. 64, no. 36, pp. 8553-8557, 2008.

[67] Y. Sun and H. C. Zhou, "Recent progress in the synthesis of metal-organic frameworks," Sci. Technol. Adv. Mat., vol. 16, no. 5, pp. 1-11, 2015.

[68] G. Lu, C. Cui, W. Zhang, Y. Liu, and F. Huo, "Synthesis and self-assembly of monodispersed metal-organic framework microcrystals," Chem. - An Asian J., vol. 8, no. 1, pp. 69-72, 2013.

[69] W. Massa, Crystal Stucture Determiantion. Germany: Springer-Verlag Berlin, 2000.

[70] J. R. F. Rouquerol, Adsorption by Powders and Porous Solids. London: Academic Press, 1999.

[71] I. D. Rahmawati, R. Ediati, and D. Prasetyoko, "Synthesis of UiO-66 using solvothermal method at high temperature," IPTEKJ. Proceeding Ser., vol. 1, pp. 2354-6026, 2014.

[72] Y. Han, M. Liu, K. Li, Y. Zuo, Y. Wei, S. Xu, G. Zhang, C. Song, Z. Zhang, and X. Guo, "Facile synthesis of morphology and size-controlled zirconium metal-organic framework UiO-66: the role of hydrofluoric acid in crystallization," Cryst. Eng. Comm, vol. 17, no. 66, pp. 6434-6440, 2015.

[73] M. Sindoro, A.-Y. Jee, and S. Granick, "Shape-selected colloidal MOF crystals for aqueous use," Chem. Commun., vol. 49, no. 83, pp. 9576-9578, 2013.

[74] J. Zhuang, C. H. Kuo, L. Y. Chou, D. Y. Liu, E. Weerapana, and C. K. Tsung, "Optimized metal-organic-framework nanospheres for drug delivery: Evaluation of smallmolecule encapsulation," ACS Nano, vol. 8, no. 3, pp. 2812-2819, 2014.

[75] G. Majano and J. Pérez-Ramírez, "Room temperature synthesis and size control of HKUST-1," Helv. Chim. Acta, vol. 95, no. 11, pp. 2278-2286, 2012.

[76] M. Zhu, S. R. Venna, J. B. Jasinski, and M. A. Carreon, "Room-temperature synthesis of ZIF-8: The coexistence of ZnO nanoneedles," Chem. Mater., vol. 23, no. 16, pp. 35903592, 2011.

[77] M. R. Destefano, T. Islamoglu, S. J. Garibay, J. T. Hupp, and O. K. Farha, "RoomTemperature Synthesis of Ui0-66 and Thermal Modulation of Densities of Defect 
Sites," Chem. Mater., vol. 29, no. 3, p. 1357-1361, 2017.

[78] H. Noh, C. Kung, T. Islamoglu, A. W. Peters, Y. Liao, P. Li, S. J. Garibay, X. Zhang, M. R. DeStefano, J. T. Hupp, O. K. Farha, "Room temperature synthesis of an 8-connected ZrBased metal - organic framework for top-down nanoparticle encapsulation," Chem. Mater., vol. 30, no. 7, pp. 2193-2197, 2018.

[79] M. R. Destefano M. R. DeStefano, T. Islamoglu, S. J. Garibay, J. T. Hupp, and O. K. Farha, "Room-temperature synthesis of UiO-66 modulation of densities of defect sites," Chem. Mater., vol. 29, no. 3, pp. 1357-1361, 2017.

[80] M. J. Katz, J. Brown, Y. J. Colo'n, P. W. Siu, K. A. Scheidt, R. Q. Snurr, J. T. Hupp, and O. K. Farha, "A facile synthesis of UiO-66, UiO-67 and their derivatives," Chem. Commun., vol. 49, no. 82, p. 9449, 2013.

[81] M. B., D. C. S., and B. G., "Influence of time on crystal attrition in a stirred vessel," AIChE J., vol. 42, no. 12, pp. 3554-3558, 2018.

[82] H. J. Scheel, "Accelerated crucible rotation: A novel stirring technique in hightemperature solution growth," J. Cryst. Growth, vol. 13-14, no. C, pp. 560-565, 1972.

[83] H. Arend and J. Hulliger, Crystal growth in science and technology. 1990.

[84] M. A. C. S.R. Venna, J. B. Jasinski, "Structural evolution of zeolitic imidazolate framework-8(ZIF-8)," J. Am. Chem. Soc., vol. 132, no. 51, pp. 18030-18033, 2010.

[85] M. A. Cheney, P. K. Bhowmik, S. Moriuchi, M. Villalobos, S. Qian, and S. W. Joo, "The Effect of Stirring on the Morphology of Birnessite Nanoparticles," J. Nanomater., vol. 2008, pp. 1-9, 2008.

[86] A. G. Gomez, G. De Silveira, H. Doan, and C. H. Cheng, "A facile method to tune zeolite L crystals with low aspect ratio," Chem. Commun., vol. 47, no. 20, pp. 5876-5878, 2011.

[87] J. Ren, H. W. Langmi, B. C. North, M. Mathe, and D. Bessarabov, "Modulated synthesis of zirconium-metal organic framework (Zr-MOF) for hydrogen storage applications," Int. J. Hydrogen Energ., vol. 39, no. 2, pp. 890-895, 2014.

[88] T. A. Mulyati, R. Ediati, and A. Rosyidah, "Influence of solvothermal temperatures and times on crystallinity and morphology of MOF-5," Indones. J. Chem., vol. 15, no. 2, pp. 101-107, 2015.

[89] F. Millange, R. El Osta, M. E. Medina, and R. I. Walton, "A time-resolved diffraction study of a window of stability in the synthesis of a copper carboxylate metal-organic framework," Cryst. Eng. Comm., vol. 13, pp. 103-108, 2011.

[90] P. Chlubná W. J. Roth, H. F. Greer, W. Zhou, O. Shvets, A. Zukal, J. Čejka, and R. E. Morris, "3D to 2D routes to ultrathin and expanded zeolitic materials," Chem. Mater., vol. 25, no. 4, p. 542-547, 2013.

[91] W. J. Roth, P. Nachtigall, R. E. Morris, and J. Čejka, "Two-dimensional zeolites: Current status and perspectives," Chem. Rev., vol. 114, no. 9, pp. 4807-4837, 2014. 
[92] W. J. Roth, P. Nachtigall, R. E. Morris, and J. Čejka, "A family of zeolites with controlled pore size prepared using a top-down method," Nat. Chem., vol. 5, pp. 628-633, 2013.

[93] M. Ogura, Y. Kawazu, H. Takahashi, and T. Okubo, "Aluminosilicate species in the hydrogel phase formed during the aging process for the crystallization of FAU zeolite," Chem. Mater., vol. 15, no. 13, pp. 2661-2667, 2003.

[94] P. S. Wheatley P. S. Wheatley, P. Chlubná-Eliášová, H. Greer, W. Zhou, V. R. Seymour, D. M. Dawson, S. E. Ashbrook, A. B. Pinar, L. B. McCusker, M. Opanasenko, J. Čejka, and R. E. Morris, "Zeolites with continuously tuneable porosity," Angew. Chemie Int. Ed., vol. 53, pp. $13210-13214,2014$.

[95] M. Kondo, Y. Takashima, J. Seo, S. Kitagawa, and S. Furukawa, "Control over the nucleation process determines the framework topology of porous coordination polymers," Cryst. Eng. Comm., vol. 12, no. 8, pp. 2350-2353, 2010.

[96] P. X. Yin, J. Zhang, Y. Y. Qin, J. K. Cheng, Z. J. Li, and Y. G. Yao, "Role of molar-ratio, temperature and solvent on the $\mathrm{Zn} / \mathrm{Cd}$ 1,2,4-triazolate system with novel topological architectures," Cryst. Eng. Comm., vol. 13, no. 10, pp. 3536-3544, 2011.

[97] Y. P. Wu D. Li, F. Fu, W. Dong, J. Zhao, K. Zou, and Y. Wang, "Stoichiometry of N-donor ligand mediated assembly in the Zn II-Hfipbb system: From a 2-fold interpenetrating pillared-network to unique $(3,4)$-connected isomeric nets," Cryst. Growth Des., vol. 11, pp. 3850-3857, 2011.

[98] C. Zou, S. Vagin, A. Kronast, and B. Rieger, "Template mediated and solvent-free route to a variety of UiO-66 metal-organic frameworks," $R S C A d v$. , vol. 6, no. 105, pp. 102968-102971, 2016.

[99] G. C. Shearer S. Chavan, J. Ethiraj, J. G. Vitillo, S. Svelle, U. Olsbye, C. Lamberti, S. Bordiga, and K. P. Lillerud, "Tuned to perfection ironing out the defects in metal-organic framework UiO-66," Chem. Mater., vol. 26, no. 14, pp. 4068-4071, 2014.

[100] Y. X. Sun and W. Y. Sun, "Influence of temperature on metal-organic frameworks," Chinese Chem. Lett., vol. 25, no. 6, pp. 823-828, 2014.

[101] H. L. Jiang, Y. Tatsu, Z. H. Lu, and Q. Xu, "Non-, micro-, and mesoporous metal-organic framework isomers: Reversible transformation, fluorescence sensing, and large molecule separation," J. Am. Chem. Soc., vol. 132, no. 16, pp. 5586-5587, 2010.

[102] G.-X. Liu, H. Xu, H. Zhou, S. Nishihara, and X.-M. Ren, “Temperature-induced assembly of MOF polymorphs: Syntheses, structures and physical properties," Cryst. Eng. Comm, vol. 14, no. 5, p. 1856, 2012.

[103] Y. Han, M. Liu, K. Li, Y. Zuo, Y. Wei, S. Xu, G. Zhang, C. Song, Z. Zhang, and X. Guo, "Facile synthesis of morphology and size-controlled zirconium metal-organic framework UiO-66: the role of hydrofluoric acid in crystallization," Cryst. Eng. Comm, vol. 17, no. 33, pp. 6434-6440, 2015.

[104] R. Seetharaj, P. V. Vandana, P. Arya, and S. Mathew, “Dependence of solvents, pH, molar 
ratio and temperature in tuning metal organic framework architecture," Arab. J. Chem., vol. 12, no. 3, pp. 295-315, 2015.

[105] E. Biemmi, S. Christian, N. Stock, and T. Bein, "High-throughput screening of synthesis parameters in the formation of the metal-organic frameworks MOF-5 and HKUST-1," Micropor. Mesopor. Mat., vol. 117, pp. 111-117, 2009.

[106] F. Taulelle E. Breynaert, K. Asselman, E. Vaneeckhaute, S.Radhakrishnan, M. W.Anderson, F. Taulelle, M. Haouas, J. A.Martens, and C. E. A. Kirschhock, "Evolution of the crystal growth mechanism of zeolite W (MER) with temperature," Micropor. Mesopor. Mat., vol. 274, no. August 2018, pp. 379-384, 2018.

[107] P. G. Vekilov, "The two-step mechanism of nucleation of crystals in solution," Nanoscale, vol. 2, no. 11, pp. 2346-2357, 2010.

[108] S. A. Orefuwa, H. Yang, and A. J. Goudy, "Rapid solvothermal synthesis of an isoreticular metal-organic framework with permanent porosity for hydrogen storage," Micropor. Mesopor. Mat., vol. 153, pp. 88-93, 2012.

[109] S. Kitagawa, "Controlled multiscale synthesis of porous coordination polymer in nano/micro regimes," Chem. Mater., vol. 22, no. 16, pp. 4531-4538, 2010.

[110] H. Wu Y. S. Chua, V. Krungleviciute, M. Tyagi, P. Chen, T. Yildirim and W. Zhou, "Unusual and highly tunable missing-linker defects in zirconium metal - organic framework UiO-66 and their important effects on gas adsorption," J. Am. Chem. Soc., vol. 135, no. 28, pp. 10525-10532, 2013.

[111] C. M. Phan and H. M. Nguyen, "Role of capping agent in wet synthesis of nanoparticles," J. Am. Chem. Soc., vol. 121, no. 17, pp. 3213-3219, 2017.

[112] D. Zacher, R. Schmid, C. Wöll, and R. A. Fischer, "Surface chemistry of metal-organic frameworks at the liquid-solid interface," Angew. Chemie Int. Ed., vol. 50, no. 1, pp. 176199, 2011.

[113] B. Van De Voorde I. Stassen, B. Bueken, F. Vermoortele, D. D. Vos, R. Ameloot, J. Tan, and T. D. Bennet, "Improving the mechanical stability of zirconium-based metalorganic frameworks by incorporation of acidic modulators," J. Mater. Chem. A, vol. 3, pp. 1737-1742, 2015.

[114] K. P. Lillerud, S. Bordiga, S. Svelle, S. Chavan, G. C. Shearer, and U. Olsbye, "Defect engineering: tuning the porosity and composition of the metal-organic framework uio-66 via modulated synthesis," Chem. Mater., vol. 28, no. 11, pp. 3749-3761, 2016.

[115] M. G. Goesten, F. Kapteijn, and J. Gascon, "Fascinating chemistry or frustrating unpredictability: Observations in crystal engineering of metal-organic frameworks," Cryst. Eng. Comm., vol. 15, no. 45, pp. 9249-9257, 2013.

[116] D. Banerjee, A. Smirnov, P. M. Forster, L. A. Borkowski, S. J. Teat, and J. B. Parise, "Synthesis and structural characterization of magnesium based coordination networks in different solvents," Cryst. Growth Des., vol. 11, no. 6, pp. 2572-2579, 2011. 
[117] J. Li, J. Sculley, and H. Zhou, "Metal-Organic Frameworks for Separations," Chem. Rev., vol. 112, no. 2, pp. 869-932, 2012.

[118] S. Friebe, B. Geppert, F. Steinbach, and J. Caro, "Metal-Organic Framework UiO-66 Layer: A Highly Oriented Membrane with Good Selectivity and Hydrogen Permeance," ACS Appl. Mater. Inter., vol. 9, no. 14, pp. 12878-12885, 2017.

[119] B. Shan J. B. James, M. R. Armstrong, E. C. Close, P. A. Letham, K. Nikkhah, Y. S. Lin, and $\mathrm{B}$. $\mathrm{Mu}$, "Influences of deprotonation and modulation on nucleation and growth of UiO-66: Intergrowth and Orientation," J. Phys. Chem. C, vol. 122, no. 4, pp. 2200-2206, 2018.

[120] Z. Li, Y. Cui, Y. Liu, C. Tan, and X. Han, "Controlled Exchange of Achiral Linkers with Chiral Linkers in Zr-Based UiO-68 Metal-Organic Framework," J. Am. Chem. Soc., vol. 140, no. 47, pp. 16229-16236, 2018.

[121] Y. Liu, G. Zeng, Y. Pan, and Z. Lai, "Synthesis of highly c-oriented ZIF-69 membranes by secondary growth and their gas permeation properties," J. Memb. Sci., vol. 379, no. 12, pp. 46-51, 2011.

[122] Y. Zhao, Z. Lv, F. Song, Q. Zhong, and Y. Cao, "Preparation and enhanced CO2 adsorption capacity of UiO-66/graphene oxide composites," J. Ind. Eng. Chem., vol. 27, pp. 102107, 2014.

[123] J. B. DeCoste, T. J. Demasky, M. J. Katz, O. K. Farha, and J. T. Hupp, "A UiO-66 analogue with uncoordinated carboxylic acids for the broad-spectrum removal of toxic chemicals," New J. Chem., vol. 39, no. 4, pp. 2396-2399, 2015.

[124] S. Šašic, T. Amari, H. W. Siesler, and Y. Ozaki, "Polycondensation reaction of bis(hydroxyethylterephthalate) - Self modeling curve resolution analysis of on-line ATR/FT-IR spectra," Appl. Spectrosc., vol. 55, no. 9, pp. 1181-1191, 2001.

[125] M. B. Shundalau, P. S. Chybirai, A. I. Komyak, A. P. Zazhogin, M. A. Ksenofontov, and D. S. Umreiko, "modeling of structures and calculation of ir vibrational spectra of N,Ndimethylformamide dimers by density functional theory," J. Appl. Spectrosc., vol. 78, no. 3, pp. 326-336, 2011.

[126] A. R. H. COLE, International union of pure and applied chemistry commission on molecular structure and spectroscopy tables of wavenumbers for the calibration of infrared spectrometers, 2nd ed. 1977.

[127] Y. Zhao, X. Mu, C. Bao, Y. Fan, J. Zhang, and Y. Wang, "Alkyl chain length dependent morphology and emission properties of the organic micromaterials based on fluorinated quinacridone derivatives," vol. 25, no. 5, pp. 3264-3270, 2009.

[128] M. M. Heravi, M. Ghavidel, and L. Mohammadkhani, "Beyond a solvent: Triple roles of dimethylformamide in organic chemistry," $R S C A d v$., vol. 8, no. 49, pp. 27832-27862, 2018.

[129] E. Hermansson, "Synthesis of a magnetic catalyst for depolymerisation of polyester," (Master's Thesis in Material Chemistry), Chalmers University of Technology, 2016. 
[130] F. Awaja and D. Pavel, "Recycling of PET," Eur. Polym. J., vol. 41, no. 7, pp. 1453-1477, 2005.

[131] B. Schmid, M. Döker, and J. Gmehling, "Esterification of ethylene glycol with acetic acid catalyzed by Amberlyst," Ind. Eng. Chem. Res., vol. 47, no. 3, pp. 698-703, 2008.

[132] M. U. Anwar Y. Lan, L. M. C. Beltran, R. Clérac, S. Pfirrmann, C. E. Anson, and A. K. Powell, "In situ ligand transformation in the synthesis of manganese complexes: mono-, triand a barrel-shaped tetradeca-nuclear MnII4 aggregate," Inorg. Chem., vol. 48, no. 12, pp. 5177-5186, 2009.

[133] P. Zikolov and O. Budevsky, "Acid-base equilibria in ethylene glycol-I Definition of $\mathrm{pH}$ and determination of pk-values of acid-base indicators," Talanta, vol. 20, no. 5, pp. 487-493, 1973.

[134] O. Hernandez, "Terephthalic Acid (TPA): SIDS initial assessment report for 12th SIAM," 100-21-0, pp. 1-82, UNEP Publ., Washington, DC, U.S.A., 2001.

[135] W. Morris S. Wang, D. Cho, E. Auyeung, P. Li, O. K. Farha, and C. A. Mirkin, "Role of modulators in controlling the colloidal stability and polydispersity of the UiO-66 metal-organic framework," ACS Appl. Mater. Inter., vol. 9, no. 39, pp. 33413-33418, 2017.

[136] Wahiduzzaman, K. Allmond, J. Stone, S. Harp, and K. Mujibur, "Synthesis and electrospraying of nanoscale mof (metal organic framework) for high-performance co2 adsorption membrane," Nanoscale Res. Lett., vol. 12, no. 1, pp. 1-12, 2017.

[137] N. Li, J. Xu, R. Feng, T. L. Hu, and X. H. Bu, "Governing metal-organic frameworks towards high stability," Chem. Commun., vol. 55, no. 52, pp. 8501-8513, 2016.

[138] A. Nimmermark, L. Öhrström, and J. Reedijk, "Metal-ligand bond lengths and strengths: Are they correlated? A detailed CSD analysis," Z. Kristallogr., vol. 228, no. 7, pp. 311317, 2013.

[139] R. Valenzuela, M. C. Fuentes, C. Parra, J. Baeza, N. Duran, S. K. Sharma, M. Knobel, J. Freer, "Influence of stirring velocity on the synthesis of magnetite nanoparticles $\left(\mathrm{Fe}_{3} \mathrm{O}_{4}\right)$ by the co-precipitation method," J. Alloys Compd., vol. 488, no. 1, pp. 227-231, 2009.

[140] M. Kandiah M. H. Nilsen, S. Usseglio, S. Jakobsen, U. Olsbye, M. Tilset, C. Larabi, E. A. Quadrelli, F. Bonino, and K. P. Lillerud, "Synthesis and stability of tagged UiO-66 ZrMOFs," Chem. Mater., vol. 22, no. 24, pp. 6632-6640, 2010.

[141] M. J. Cliffe W. Wan, X. Zou, P. A. Chater, A. K. Kleppe, M. G. Tucker, H. Wilhelm, N. P. Funnell, F. Coudert, and A. L. Goodwin, "Correlated defect nanoregions in a metalorganic framework," Nat. Commun., vol. 5, no. May, pp. 1-8, 2014.

[142] Q. Chu, G. X. Liu, T. aki Okamura, Y. Q. Huang, W. Y. Sun, and N. Ueyama, "Structure modulation of metal-organic frameworks via reaction $\mathrm{pH}$ : Self-assembly of a new carboxylate containing ligand $\mathrm{N}$-(3-carboxyphenyl)iminodiacetic acid with cadmium(II) and cobalt(II) salts," Polyhedron, vol. 379, no. 1-2, pp. 46-51, 2008. 
[143] L.-S. Long, "pH effect on the assembly of metal-organic architectures," Cryst. Eng. Comm, vol. 12, no. 5, p. 1354-1365, 2010. 